\title{
Manuale di Conservazione Digitale
}

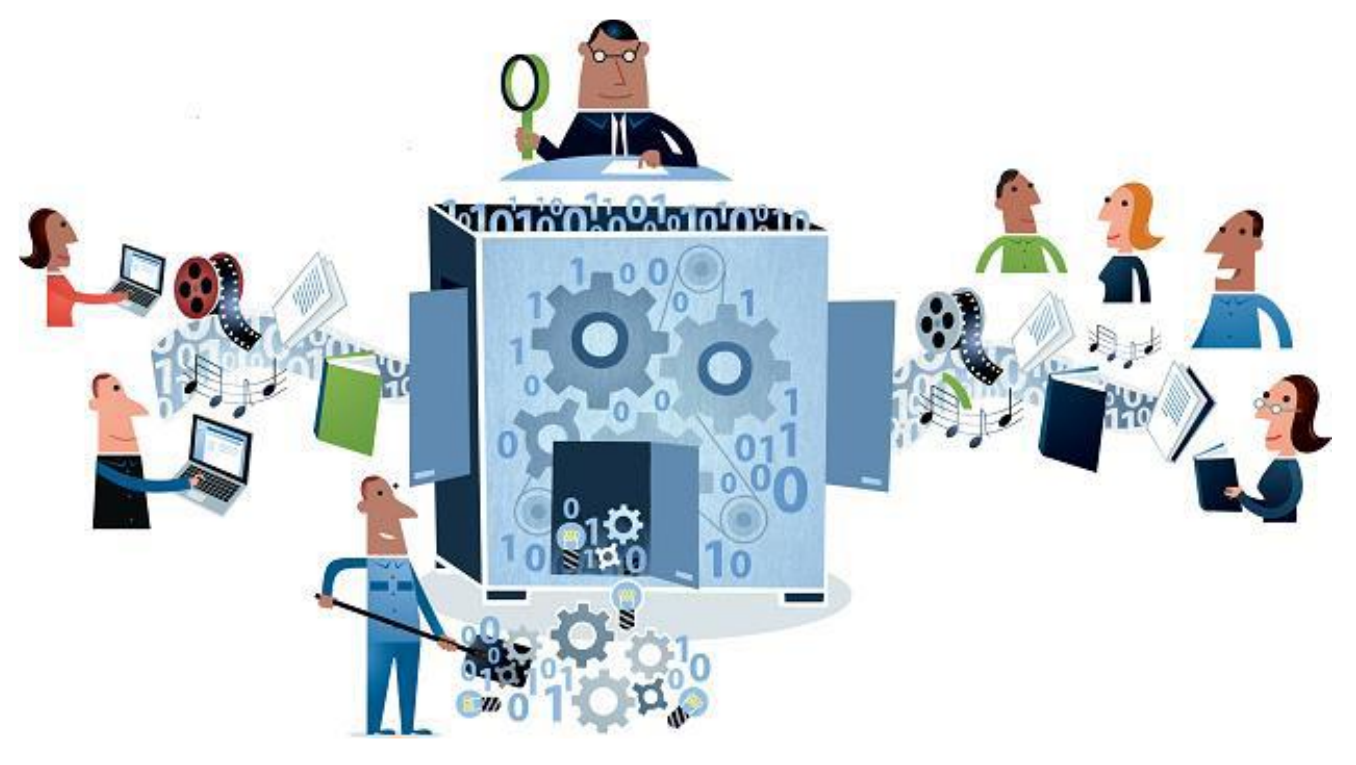

Illustrazione di Jørgen Stamp digitalbevaring.dk CC BY 2.5 Denmark 
Benvenuti nella seconda versione aggiornata del Manuale di Conservazione Digitale. Una base di conoscenza essenziale per la conservazione digitale, revisionata e accessibile gratuitamente da tutti.

"Overall the improvements to the Handbook make it, in my opinion, one of the more useful and flexible tools for identifying, understanding and getting to grips with practical approaches to the varying challenges of digital preservation. It uses approachable language, clear terminology and provides useful links to case studies and further reading which will be of benefit to students and practitioners alike." Stefanie Davidson West Yorkshire Archive Service

L'informazione digitale è divenuta sempre più importante nella nostra cultura. II Manuale, elaborato in un primo momento nel 2001 da Neil Beagrie e Maggie Jones, viene gestito e aggiornato dalla Digital Preservation Coalition (DPC). Questa versione completa (Seconda Edizione) ha ampliato e aggiornato il contenuto con 30 sezioni principali (si veda l'Indice). La Seconda Edizione è stata realizzata con il contributo di 45 professionisti ed esperti del settore della conservazione digitale sotto la direzione dei Consigli di Amministrazione e Comitati Consultivi. II Manuale offre una guida autorevole e pratica a livello internazionale relativa alla gestione nel tempo delle risorse digitali e alle questioni relative al loro accesso. Sarà d'interesse per tutti coloro che sono coinvolti nella creazione e gestione di risorse digitali.

\section{Informazioni sulla traduzione italiana}

La traduzione italiana è stata realizzata dal Laboratorio di Documentazione - Dipartimento di Culture, Educazione e Società dell'Università della Calabria.

II Manuale è stato tradotto da Assunta Caruso e Claudia Lanza. La premessa all'edizione italiana è di Roberto Guarasci.

II manuale è stato relizzato per essere utilizzato in un ambito normativo e regolamentare anglosassone. Per un corretto uso in Italia si consiglia di consultare, in parallelo, le Linee Guida sulla Formazione, Gestione e Conservazione dei Documenti Informatici (https://trasparenza.agid.gov.it/archivio19 regolamenti 0 5385.html)

\section{Riutilizzare queste informazioni}

È possibile riutilizzare questo materiale in lingua inglese (ad eccezione dei loghi), con la necessità di integrare gratuitamente ringraziamenti in qualsiasi formato o con qualsiasi mezzo. Per i dettagli completi sulle autorizzazioni e sui ringraziamenti per il riutilizzo si veda la sezione $\underline{\text { Come usare il Manuale. }}$

Per autorizzazioni alla traduzione in altre lingue si contatti per e-mail: 
handbook@dpconline.org

Si prega di usare la seguente citazione per il Manuale: Digital Preservation Handbook, 2nd Edition, https://www.dpconline.org/handbook, Digital Preservation Coalition (c) 2015. 
La Conservazione dei Documenti Informatici nel contesto italiano...................................................... 6

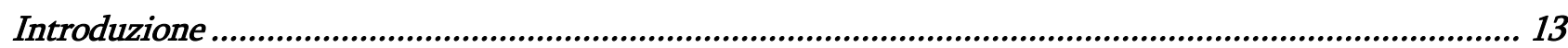

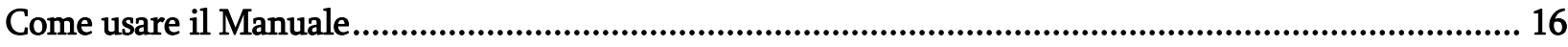

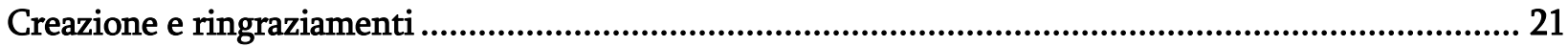

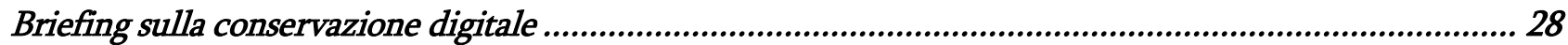

Perché è importante la conservazione digitale .......................................................................................... 29

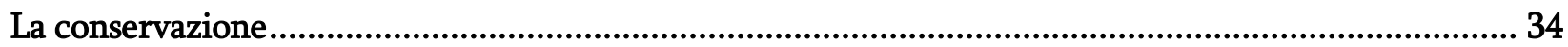

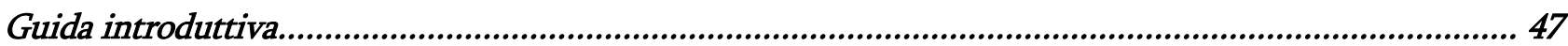

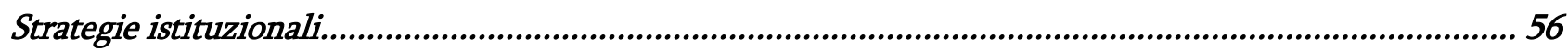

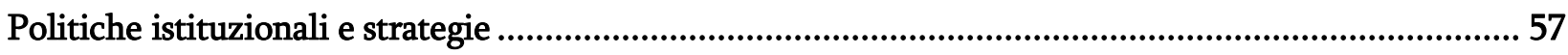

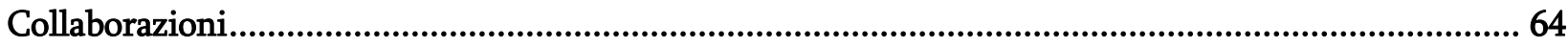

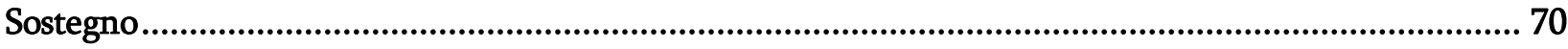

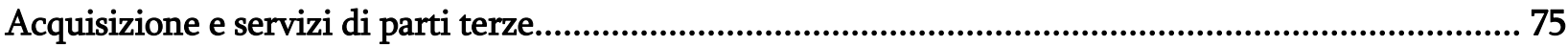

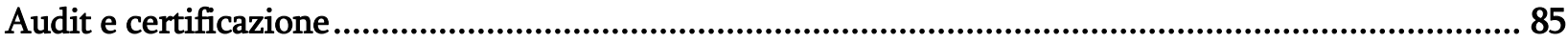

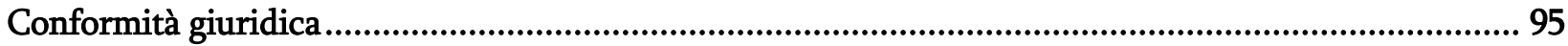

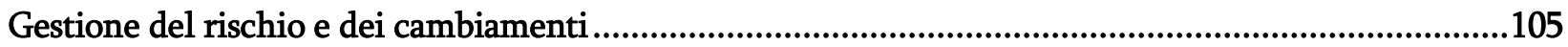

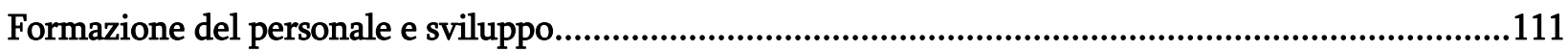

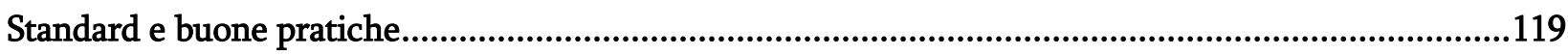

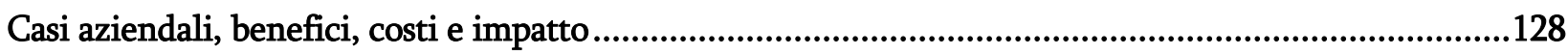

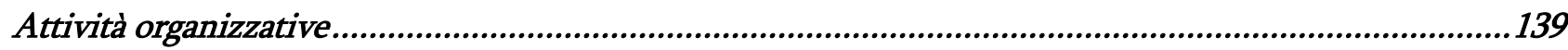

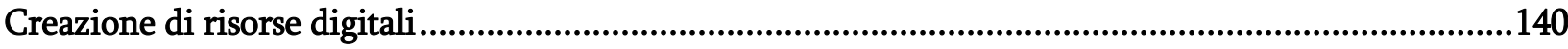

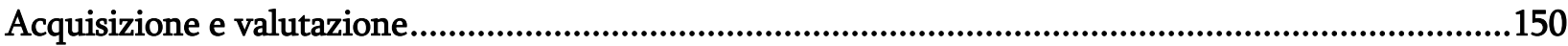

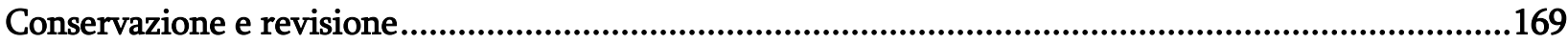

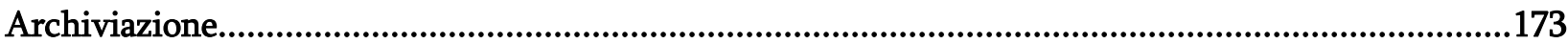

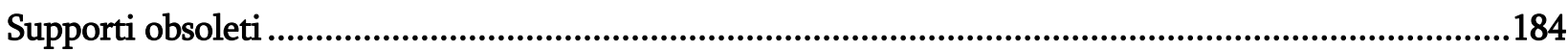

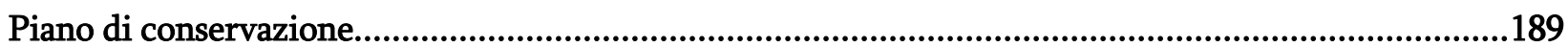

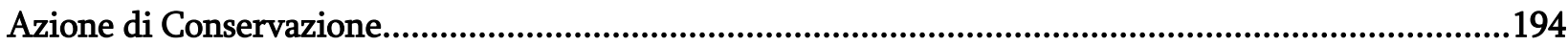

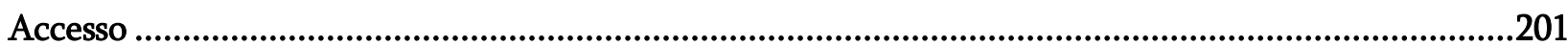

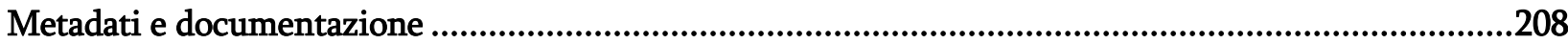




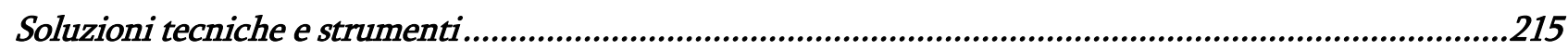

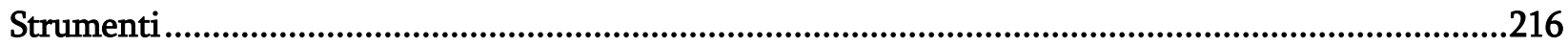

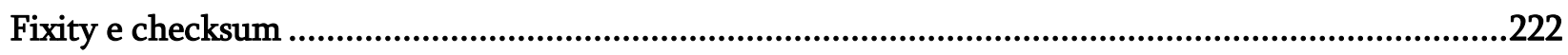

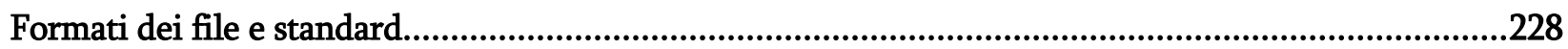

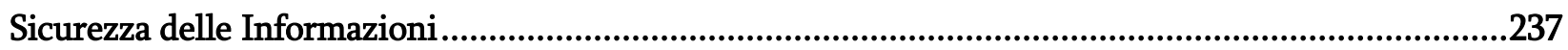

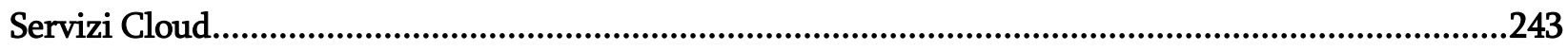

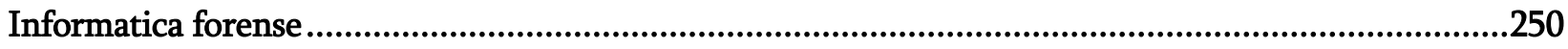

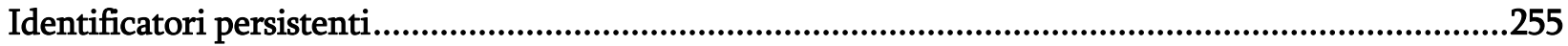

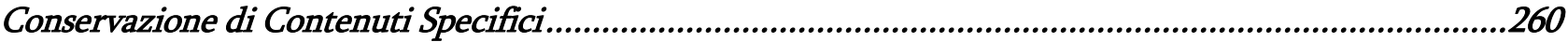

Riviste on line

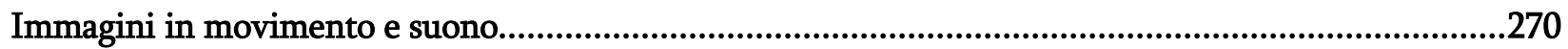

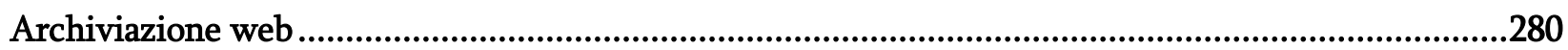

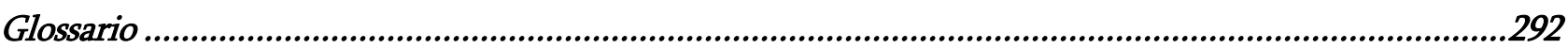

Aggiornamenti e integrazioni verranno effettuati regolarmente. Ogni nuova sezione prevista nelle future pubblicazioni del DPC sarà indicata come "in progress". 


\title{
La Conservazione dei Documenti Informatici nel contesto italiano
}

\section{Premessa all'edizione italiana del manuale della Conservazione}

Negli ultimi decenni il numero di lavori scientifici o presunti tali sul digitale e la sua gestione e conservazione sono, in Italia, esponenzialmente cresciuti. All'equazione degli anni Novanta dello scorso secolo, digitale uguale efficienza se ne è sostituita negli anni duemila - complice anche i social - una più generica ma più pervasiva anche se non meno fallace, ovvero digitale uguale modernità. Da quella data non c'è stato insegnamento universitario, conferenza o convegno che a vario titolo, non inserisse - nel testo o nei programmi - uno dei numerosi termini della galassia digitale. L'emergenza pandemica della primavera 2020 ha poi costretto ad un uso massivo del digitale che è diventato necessità impellente per il funzionamento dei servizi essenziali.

A quella data il Paese ha scoperto ciò che era noto da tempo ovvero che il processo di transizione al digitale, nel pubblico e nel privato, era stato non guidato, a macchia di leopardo e gestito dall'offerta più che dalla domanda e dai bisogni. Che l'Italia, come molti altri paesi europei, avesse problemi di connettività e di educazione all'uso delle tecnologie era noto da decenni ed evidenziato annualmente nei rapporti Istat su cittadini e nuove tecnologie che fotografavano impietosamente un paese con oltre venti milioni di non connessi ma con la più alta media di utilizzo di apparecchi telefonici mobili dell'Unione Europea1'. Su questa particolarità si sarebbe potuta costruire una strategia alternativa per garantire la connettività del Paese ma una serie di concause non lo hanno del tutto consentito. I fondi europei per il dopo pandemia e il piano nazionale di ripresa e resilienza (PNRR) correttamente collocano la trasformazione digitale al centro degli investimenti e delle strategie di rilancio declinandole in alcuni assi portanti:

\begin{abstract}
"Il pilastro digitale dei PNRR deve comprendere la razionalizzazione e digitalizzazione della pubblica amministrazione e lo sviluppo dei servizi pubblici digitali. Si deve inoltre migliorare la connettività, anche tramite un'ampia diffusione di reti di telecomunicazione (TLC) ad altissima capacità. I costi per gli utenti devono essere sostenibili e la velocità di realizzazione della rete deve essere aumentata. I Piani devono inoltre sostenere la ricerca e sviluppo $(R \& S)$ nelle TLC e l'adozione delle tecnologie digitali da parte delle imprese, in particolare delle piccole e medie. Le competenze digitali di cittadini e lavoratori devono aumentare, così come la loro capacità di accesso a strumenti e servizi digitali, particolarmente per i gruppi sociali vulnerabili. Gli investimenti digitali devono essere allineati alle comunicazioni della Commissione in materia. Devono essere evidenziate e valorizzate le sinergie tra investimenti verdi e digitali.2"
\end{abstract}

Il dettaglio degli investimenti, ampiamente descritti nel prosieguo del piano, declina quanto anticipato in premessa: infrastrutture digitali, abilitazione e facilitazione migrazione al cloud, dati e interoperabilità, servizi digitali e cittadinanza digitale, cybersecurity, digitalizzazione delle

1 https://www.istat.it/it/archivio/251968, consultato il 27.09.2021.

2 https://www.governo.it/sites/governo.it/files/PNRR.pdf, consultato il 27.09.2021. 
grandi amministrazioni centrali, competenze digitali di base, processo di acquisto ICT, supporto alla trasformazione della pubblica amministrazione (PA) locale, introduzione linee guida cloud first e interoperabilità. II termine o il concetto di conservazione del digitale non compare mai mentre compare più volte il termine di de-materializzazione applicato a vari domini, dalla giustizia alla logistica, inteso nel senso di una transizione rapida dei documenti da supporto analogico a digitale con un punto determinato dal quale partire senza curarsi troppo del pregresso. L'accento è posto sull'attualità del documento digitale lasciando nel vago la sua gestione e conservazione nel tempo anche sulla base di un mai chiarito equivoco tra dato e documento. Ampia è la frequenza terminologica di dato/i spesso usato/i come sinonimo d'uso di documento/i specie quando si parla della pubblica amministrazione.

Prima di entrare più nello specifico per evitare di incorrere in equivoci dai quali non rifuggono nemmeno gli addetti ai lavori è opportuno - a questo punto - fare una breve digressione terminologica anche in relazione a quanto di cui sopra. II termine "dematerializzazione" indica l'attività di transizione da un supporto analogico ad uno digitale di documenti che hanno nell'analogico l'originale con valore legale. L'attività non è obbligatoria e va attentamente valutata in relazione al rapporto costi benefici per l'amministrazione produttrice e/o detentrice del patrimonio documentale oggetto di intervento ${ }^{3}$. La "conservazione dei documenti informatici" è l'attività di conservazione dei documenti nativi digitali che hanno nell'originale digitale l'esemplare con valore legale ${ }^{4}$. Si tratta dei documenti correnti necessari per il corretto e quotidiano funzionamento delle amministrazioni produttrici. Essi fin dal loro nascere uniscono alla valenza amministrativa quella storico culturale in quanto individuati come beni culturali ${ }^{5}$. Questa compresenza di valenza amministrativa/bene culturale se, da un lato, aumenta il livello di tutela dall'altro ipotizza una necessaria sinergia tra amministrazioni diverse non sempre facile da gestire.

L'articolo 4.1 delle Linee Guida sulla formazione, gestione e conservazione dei documenti informatici (d'ora in poi Linee Guida) prescrive che sono da conservare i documenti, di solito raggruppati nell'unità archivistica primaria, il fascicolo, che normalmente coincide con un singolo affare, pratica o procedimento amministrativo, le serie e le aggregazioni documentali informatiche complete dei rispettivi metadati 6 . L'indicazione segue la teoria archivistica classica dei documenti analogici secondo la quale il legame organico che collega i documenti e/o le singole unità archivistiche tra loro non è solo un elemento meccanico di condizionamento ma è apportatrice di ulteriori elementi culturali di contesto che però hanno pienezza di significato

\footnotetext{
3 Art. 42 Decreto Legislativo 82/2005.
}

${ }^{4}$ Art. 43 Decreto Legislativo 82/2005. Per il concetto di originale dei documenti digitali si veda: Guarasci R., Rovella A., Pasceri E., Caruso A., II concetto di originale di un documento digitale nelle intercettazioni di comunicazioni nel processo penale, in JLIS.IT, vol. 10 (2), 2019, pp. 28-36, DOI:10.4403/jlis.it-12506.

5 Art. 10 Decreto Legislativo 42/2004.

6 "Il sistema di conservazione assicura, dalla presa in carico fino all'eventuale scarto, la conservazione dei seguenti oggetti digitali in esso conservati, tramite l'adozione di regole, procedure e tecnologie, garantendone le caratteristiche di autenticità, integrità, affidabilità, leggibilità, reperibilità:

a) i documenti informatici e i documenti amministrativi informatici con i metadati ad essi associati;

b) le aggregazioni documentali informatiche (fascicoli e serie) con i metadati ad esse associati contenenti i riferimenti che univocamente identificano i singoli oggetti documentali che costituiscono le aggregazioni medesime, nel rispetto di quanto indicato per le Pubbliche Amministrazioni nell'articolo 67, comma 2, del DPR 445/200042 e art. 44, comma 1-bis, CAD;

c) gli archivi informatici con i metadati associati”. Linee Guida, art. 4.1. 
quando il vincolo si traduceva anche in una collocazione fisica staticamente definita che evidenziava il nesso di causalità tra i singoli elementi. Quanto questo sia significativo in un contesto nel quale il documento non esiste in sé ma solo in ragione della richiesta informativa dell'utente in quanto insieme di dati organizzati secondo una struttura associata a delle regole di visualizzazione che permettono una leggibilità condivisa tra l'autore e l'utente-lettore meriterebbe una più approfondita riflessione.

Ciò anche in ragione delle stesse modalità di redazione del documento informatico definite nell'articolo 2.1.1. delle citate Linee Guida:

"1. II documento informatico è formato mediante una delle seguenti principali modalità:

a) redazione tramite l'utilizzo di appositi strumenti software;

b) acquisizione di un documento informatico per via telematica o su supporto informatico, acquisizione della copia per immagine su supporto informatico di un documento analogico, acquisizione della copia informatica di un documento analogico;

c) registrazione informatica delle informazioni risultanti da transazioni o processi informatici o dalla presentazione telematica di dati attraverso moduli o formulari resi disponibili all'utente;

d) generazione o raggruppamento anche in via automatica di un insieme di dati o registrazioni, provenienti da una o più basi dati, anche appartenenti a più soggetti interoperanti, secondo una struttura logica predeterminata e memorizzata in forma statica ${ }^{7}$.

Nel mentre le prime due modalità permettono di conservare ancora una qualche pur apparente fisicità del documento la previsione dei punti $c$ e $d$ disancora completamente il testo da qualsivoglia idea di esistenza indipendente dalla struttura logica di recupero/visualizzazione che si conferma come l'unico momento del processo nel quale possono essere dettate delle regole di corretta gestione documentale.

Ha poco senso continuare a proiettare sui documenti informatici regole e procedure nate e costruite sull'analogico per il semplice motivo che il digitale non è solo una tecnologia abilitante che può essere usata in maniera ipostatica rispetto al contenuto ma è una diversa modalità di produzione dei documenti che richiede regole e procedure specifiche e non mutuate.

Così come l'identità analogica basata sul riconoscimento antropometrico e la successiva associazione di dati anagrafici garantiti da un soggetto avente potestà di farlo è completamente diversa dall'identità digitale in quanto manca l'elemento visuale dell'operatore umano che effettua il raffronto, così il documento informatico ha la stessa valenza giuridica del documento analogico ma una struttura ed una natura completamente diverse.

II quadro normativo italiano sulla gestione e conservazione dei documenti informatici è complesso e articolato e, pur avendo sposato la logica del testo unico, ha risentito nella sua evoluzione delle diverse accezioni del digitale nella società civile e nei percorsi mentali dei decisori.

\footnotetext{
7 Agenzia per l'Italia Digitale, Linee guida sulla formazione, gestione e conservazione dei documenti
} informatici, settembre 2020. 
Il punto di partenza che ha poi permesso tutte le evoluzioni successive è la definizione di documento data dall'art. 22 dalla legge $241 / 90^{8}$ che, configurando il documento come rappresentazione di atti o fatti rilevanti per l'amministrazione, lo ha svincolato da qualsivoglia supporto, connaturato correttamente come semplice elemento occasionale utilizzato per la redazione. La definizione, con minime modifiche, viene integralmente ripresa nel DPR 445/2000 e poi nel Decreto Legislativo 82/2005.

II Decreto del Presidente della Repubblica 28 dicembre 2000 n. 445 "Testo Unico delle disposizioni legislative e regolamentari in materia di documentazione amministrativa" (d'ora in poi Testo Unico) dopo una intensa quanto disorganica produzione normativa degli anni novanta nella quale merita di essere ricordato il Decreto Legislativo 39/1993 che prescriveva, all'art. 3.1, che "Gli atti amministrativi adottati da tutte le pubbliche amministrazioni sono di norma predisposti tramite i sistemi informativi automatizzati", propone poi una sistematizzazione organica delle norme sulla gestione documentale e sulla strutturazione organizzativa a supporto di quelle attività.

Agli inizi del nuovo millennio tutta l'attività di digitalizzazione della pubblica amministrazione e con essa tutta la gestione documentale che, riduttivamente, ne viene considerata un sottoinsieme è vista come la leva principale per ridare efficienza e competitività ad una struttura burocratica della quale si avvertono sempre più marcatamente i limiti. L'equivalenza, semplificata dai media, è: digitale uguale efficienza, carta uguale lentezza e inefficienza. In breve tempo si verificherà l'erroneità del paradigma ricordando che i processi devono essere resi efficaci prima di applicare tecnologie abilitanti che ne migliorano l'efficienza. Diversa sarà l'evoluzione nel mondo privato dove la spinta sarà innegabilmente maggiore ma una normativa molto meno puntuale di quella del mondo pubblico favorirà il nascere di soluzioni non sempre omogenee.

II 2005 vede la luce il Decreto Legislativo n. 85 Codice dell'Amministrazione Digitale (d'ora in poi CAD) con l'intento dichiarato di riunire tutta la produzione normativa sull'argomento evitando sovrapposizioni e contraddizioni tra i vari testi ma con anche la conseguenza di una necessaria generalizzazione delle disposizioni che, oltre a necessitare di regole tecniche di applicazione, ha fatto spesso percepire il corpus delle norme sul digitale come estranee alla legislazione ordinaria e quindi, di fatto, di rango inferiore e quasi accessorie anche perché prive di momenti sanzionatori.

Le regole tecniche, previste dall'art. 71 del CAD, rappresenteranno per molti anni il vero collo di bottiglia per la reale applicabilità delle norme a causa delle difficoltà di redazione connesse alla molteplicità degli attori coinvolti ${ }^{9}$. Quelle attuative del decreto legislativo 235/2010, primo

\footnotetext{
8 "Per "documento amministrativo", ogni rappresentazione grafica, fotocinematografica, elettromagnetica o di qualunque altra specie del contenuto di atti, anche interni o non relativi ad uno specifico procedimento, detenuti da una pubblica amministrazione e concernenti attività di pubblico interesse, indipendentemente dalla natura pubblicistica o privatistica della loro disciplina sostanziale”. Art. 22, comma d, legge 7 agosto 1990, n.241.

9 Nel 2005 l'organismo incaricato della materiale redazione delle Regole Tecniche è il CNIPA, Centro nazionale per l'informatica nella pubblica amministrazione, nato nel luglio del 2003 subentrando all'AIPA (costituita nel 1993), che il $1^{\circ}$ gennaio 2004, assorbì anche il Centro tecnico per la RUPA (costituito nel 1997). Nel 2009 viene riorganizzato con il nuovo nome di DigitPA diventando poi, nel 2012, Agenzia per l'Italia Digitale (AgID), agenzia tecnica della Presidenza del Consiglio dei Ministri.
} 
aggiornamento organico del Codice, avrebbero dovuto essere pubblicate entro un anno dall'entrata in vigore della norma. In effetti vennero pubblicate tra febbraio 2013 e novembre 2014 lasciando per ultimo il DPCM sulla formazione del documento informatico che era propedeutico a tutti gli altri.

Un primo correttivo si tenterà di metterlo in essere con l'art. 56 del Decreto Legislativo 179/2016 che demanderà ad un decreto del ministro delegato per la Semplificazione e la Pubblica Amministrazione e non più a un decreto del Presidente del Consiglio dei Ministri le successive emanazioni e poi con l'art. 66 del Decreto Legislativo 217/17 che sostituirà le Regole Tecniche con Linee Guida ${ }^{10}$.

Con il Decreto Legge 16 luglio 2020 n. $76^{11}$ sono state apportate ulteriori modifiche al Codice per ciò che riguarda la vigilanza sulle aziende che esercitano l'attività di conservazione dei documenti digitali.

L'art. 44 del CAD al comma 1-quater prevedeva la possibilità di affidamento all'esterno del servizio di conservazione a "soggetti pubblici e privati che offrano idonee garanzie organizzative e tecnologiche e di protezione dei dati personali". L'art. 34, modificato dal citato Decreto Legge, specificando altresì - al comma 1 bis - che la qualificazione dei soggetti pubblici e privati affidatari del servizio di conservazione sarebbe stata definita in apposito regolamento da emanarsi a cura di Agid "avuto riguardo all'esigenza di assicurare la conformità dei documenti conservati agli originali nonché la qualità e la sicurezza del sistema di conservazione". L'art. 14 bis del nominato Decreto Legge sottrae ad Agid l'attività di vigilanza sui conservatori di documenti informatici eliminando contestualmente l'albo dei conservatori all'interno del quale le pubbliche amministrazioni sono tenute a scegliere i destinatari dell'affidamento del servizio.

Ad oggi circa il $75 \%$ dei soggetti pubblici italiani hanno scelto di esternalizzare l'attività di conservazione $^{12}$ con tipologie contrattuali e scelte operative non sempre rispondenti alla normativa vigente. Una semplificazione delle procedure ed una diminuzione dei vincoli in essere non è quindi priva di rischi per la documentazione amministrativa pubblica. II regolamento sui "criteri per la fornitura dei servizi di conservazione dei documenti informatici" è stato emanato nel giugno 2021 prevedendo, all'art.3, l'istituzione di uno specifico marketplace per i servizi di conservazione pur se, lo stesso articolo sancisce la non obbligatorietà dell'iscrizione e la possibilità per le amministrazioni di avvalersi anche di conservatori non iscritti.

L'affidamento all'esterno del servizio di conservazione mentre può essere configurato come una vera e propria esternalizzazione del servizio per i soggetti privati ha dei vincoli precisi per ciò che riguarda le pubbliche amministrazioni. In particolare, ai sensi del comma 1-quater del citato art. 44 del CAD è proprio il responsabile della conservazione, preventivamente nominato, che opera motivatamente la scelta di una conservazione in house o all'esterno. È da sottolineare

\footnotetext{
10 Al momento le Linee Guida sulla Formazione, Gestione e Conservazione dei Documenti Informatici sono state pubblicate da Agenzia per l'Italia Digitale nel settembre 2020.

${ }^{11}$ Legge 11 settembre 2020, n. 120, Conversione in legge, con modificazioni, del decreto-legge 16 luglio 2020 , n. 76, recante «Misure urgenti per la semplificazione e l'innovazione digitali».

12 Guarasci R., Pasceri E., Parisi F., Il processo di digitalizzazione nelle pubbliche amministrazioni: i risultati di una indagine, in ARCHIVI, vol. XIV (1), p. 77-88.
} 
comunque che la responsabilità giuridica generale sui processi di conservazione non è in alcun caso delegabile e resta in carico sempre e comunque al responsabile della conservazione.

Nel processo di conservazione, per come delineato dal CAD e dalle Linee Guida, ruolo centrale nella definizione e formalizzazione del sistema di conservazione è rappresentato dal manuale della conservazione la cui redazione, in nessun caso delegabile, è affidata al citato responsabile $^{13}$. Esso è cosa ben diversa dal manuale di conservazione dei soggetti imprenditoriali che offrono servizi di conservazione e, anche in caso di esternalizzazione del servizio, non è mai sovrapponibile anche se tra i due documenti non è operativamente opportuno che sussistano forti discrasie.

Diverso discorso, come dicevamo, per i soggetti privati nei quali l'affidamento ad un soggetto esterno del servizio di conservazione si traduce in una vera e propria esternalizzazione dell'intero processo in quanto anche il responsabile della conservazione può essere esterno all'organizzazione pur garantendo la terzietà rispetto all'affidatario del servizio di conservazione.

Il processo di conservazione per come delineato e definito nell'attuale quadro normativo e regolamentare italiano è quindi strutturato e compiutamente definito e rappresenta il punto conclusivo di un processo avviato quasi tre decenni or sono. Purtroppo l'attuazione non omogenea di alcuni degli stadi precedenti spesso ne inficia la corretta realizzazione rischiando di farlo diventare un elemento tanto autoconsistente quanto poco funzionale.

Un ecosistema della gestione e conservazione dei documenti informatici correttamente strutturato è composto da una pluralità di attori interoperanti: il responsabile della gestione documentale ${ }^{14}$ che predispone i pacchetti di versamento e dialoga con il responsabile della conservazione sulla corretta alimentazione del sistema che parte dal momento dell'ingresso del documento nell'organizzazione; il responsabile della tutela dei dati personali (DPO) ${ }^{15}$ che concorre alla definizione delle regole per la costruzione del pacchetto di esibizione, il coordinatore della gestione documentale che, se presente, dovrebbe garantire una visione d'insieme e il responsabile della transizione digitale ${ }^{16}$ che dovrebbe funzionare da interfaccia rimuovendo gli ostacoli strutturali coordinando le politiche dell'organizzazione. E poi il responsabile dei sistemi informativi per gli aspetti tecnologici, il responsabile dell'archivio storico per le politiche di versamento e tutela e tutte le altre eventuali figure con competenze delegate che concorrono al complesso processo della gestione dei documenti ${ }^{17}$.

\footnotetext{
13 "I requisiti del processo di conservazione, le responsabilità e i compiti del responsabile della conservazione e del responsabile del servizio di conservazione, e le loro modalità di interazione sono formalizzate nel manuale di conservazione del Titolare dell'oggetto della conservazione e nelle specifiche del contratto di servizio o dell'accordo. Tali modalità trovano riscontro anche nel manuale di conservazione del conservatore.". Linee Guida, art. 4.3.

14 DPR 445/2000, Art. 61, Servizio per la gestione informatica dei documenti dei flussi documentali e degli archivi

1. Ciascuna amministrazione istituisce un servizio per la tenuta del protocollo informatico, della gestione dei flussi documentali e degli archivi in ciascuna delle grandi aree organizzative omogenee individuate ai sensi dell'articolo 50. II servizio è posto alle dirette dipendenze della stessa area organizzativa omogenea.

15 Decreto Legislativo 10 agosto 2018, n. 101.

16 CAD, art. 17.

$17 \mathrm{CAD}$, art. 44, commi 1 bis $r 1$ quater.
} 
Nell'operatività quotidiana spesso le singole funzioni affidate nel tempo a funzionari e dirigenti già titolari di altre competenze hanno una operatività limitata e non ottimale condizionando variamente l'intero processo.

Il team di traduzione dell'Università della Calabria ha scelto il Manuale di Conservazione Digitale poiché lo ha valutato come un valido punto di partenza per presentare gli approcci e le raccomandazioni generali di professionisti di tutto il mondo. II team ritiene che il Manuale affronti esaurientemente le problematiche che si verificano più frequentemente nelle attività quotidiane: l'importanza dei vari attori, la necessità di identificare e sviluppare le risorse e le competenze per la gestione di molteplici attività e processi necessari per la conservazione digitale, nonché l'adozione di una definizione di conservazione digitale sufficientemente ampia da potersi applicare ai diversi aspetti del contesto italiano. Nonostante la presenza di specificità nazionali in Italia, permane la necessità di testi di riferimento sulla conservazione che favoriscano un approccio pratico e operativo in un'ottica internazionale. È per questo motivo che l'Università della Calabria ha scelto di tradurre il Manuale di Conservazione Digitale. 


\section{Introduzione}

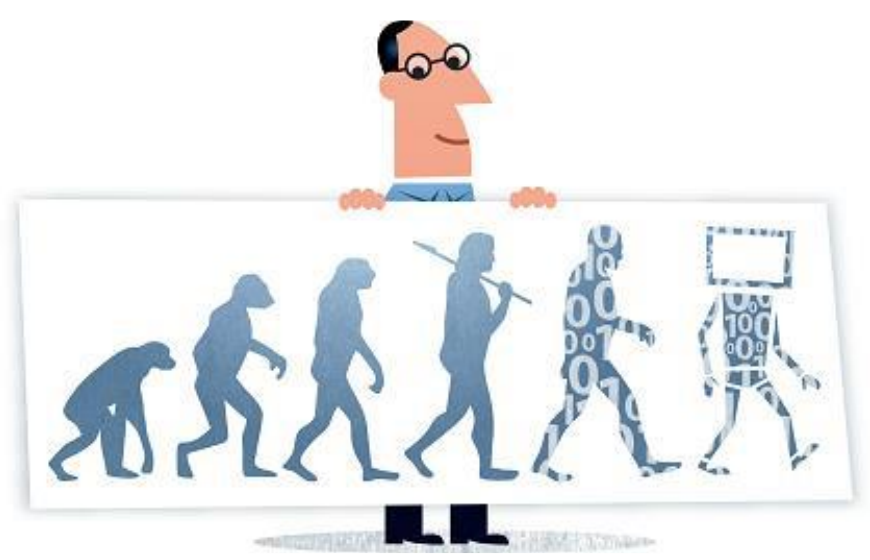

Illustrazione di Jørgen Stamp digitalbevaring.dk CC BY 2.5 Denmark

II volume delle informazioni digitali cresce sempre di più. Che si tratti del risultato di digitalizzazione di collezioni non digitali, come pubblicazioni o opere d'arte, o che sia parte delle attività ordinarie di un'organizzazione o ente privato, si assiste a una crescita sempre più elevata e veloce di informazioni. Ciò rientra in un contesto caratterizzato da una crescente consapevolezza delle importanti sfide che si presentano nel momento in cui si garantisce una forma di accesso continuo a queste risorse, anche nel breve periodo.

La combinazione di questi due fattori rappresenta sia una sfida che una difficoltà. Da una parte ci sono oggettive opportunità garantite dalla tecnologia digitale di fornire un rapido ed efficiente accesso all'informazione. Dall'altra esiste il rischio reale che le risorse digitali vengano create in maniera tale da non poter assicurare la loro accessibilità neanche per brevi periodi, e ancor meno la prospettiva che le generazioni future possano accedervi.

\section{Il bisogno di una guida}

Tutte le organizzazioni in ogni settore sono coinvolte nella produzione di risorse digitali. Queste risorse potrebbero essere create digitalizzando le collezioni non digitali al fine di consentir un più ampio accesso, oppure potrebbero essere create digitalmente ("native digitali"). Qualunque sia la modalità di creazione, esse dovranno essere gestite sin da subito, nel loro ciclo di vita, preferibilmente iniziando dalla loro fase di progettazione, o, se così non fosse, non appena tale attività diventi possibile, sempre nell'ottica che esse debbano rimanere accessibili fino a quando vi sarà richiesta di consultazione. L'esperienza concreta e le competenze in questo campo sembrano relativamente limitate, perciò c'è un chiaro bisogno di una guida per fare in modo che importanti opportunità non vengano limitate da rischi altrettanto significativi.

Questo Manuale mira a identificare le linee guida per la creazione, gestione e conservazione delle risorse digitali, nonché a fornire un insieme di strumenti pratici di supporto a questo processo. Nonostante l'esistenza di alcuni problemi, si possono individuare diversi esempi di buone pratiche e indicare delle modalità attraverso le quali le istituzioni possono iniziare a 
interessarsi di conservazione digitale. Offrendo una panoramica degli elementi principali, degli approfondimenti per strategie e attività, degli indicatori per progetti e dei report di riferimento, questo Manuale si pone l'obiettivo di fornire una guida alle istituzioni pubbliche e ai soggetti privati nonché un insieme di strumenti per aiutare questi ultimi a identificare e adottare le azioni adeguate.

\section{Pubblico e obiettivi}

La conservazione digitale presenta diversi punti in comune con la conservazione tradizionale per ciò che riguarda i principi generali, ma differisce in maniera netta a livello operativo. Di conseguenza, il pubblico di riferimento di questo Manuale è molto ampio. Innanzitutto, il Manuale mira a fornire una guida alle istituzioni a livello internazionale, nazionale, regionale e locale che sono coinvolte o che prevedono la creazione e/o l'acquisizione di risorse digitali. All'interno di queste istituzioni il Manuale si rivolge sia ad amministrazioni che a professionisti ed è strutturato in modo da includere al suo interno sia una visione strategica di alto livello che una guida operativa dettagliata. Inoltre, il Manuale è indirizzato a fornitori di servizi che potrebbero trovarsi nella posizione di fornire tutti o parte dei servizi necessari alla conservazione di risorse digitali. Infine, questo Manuale sarà d'interesse anche per gli sviluppatori di software e dei produttori di dati il cui coinvolgimento nella conservazione delle loro risorse digitali è tuttora fondamentale nonostante siano a volte limitati dalle più generali necessità aziendali delle amministrazioni di appartenenza.

Nel Manuale questi gruppi sono associati a interessi e livelli di coinvolgimento diversi in relazione alle risorse digitali e a intervalli di tempo differenti. Perseguendo un approccio legato al ciclo di vita per la conservazione digitale, il Manuale mira inoltre a supportare l'identificazione di interdipendenze, di elementi ostativi e di meccanismi di gestione al fine di agevolare la comunicazione e la collaborazione tra queste comunità.

II Manuale deve essere personalizzato nei diversi contesti d'uso in modo da poter essere utilmente impiegato dalle istituzioni, comprese quelle in cui la conservazione digitale potrebbe essere esternalizzata o a breve termine.

Le tematiche generali associate alla conservazione digitale hanno una connotazione globale, e gli esempi di buone pratiche, di attività di ricerca e di indirizzamento e di guida sono stati estratti da diversi punti nel mondo. Tuttavia, vi è una maggiore attenzione al contesto britannico e i riferimenti normativi, ad esempio, sono specificatamente quelli appartenenti al Regno Unito. È comunque auspicabile che il Manuale abbia rilevanza a livello internazionale dal momento che i diversi modelli e i riferimenti forniti non sono esclusivamente inglesi, e sono, in ogni caso, applicabili a qualsiasi Paese. Qualunque sia il Paese d'origine, gli utenti che utilizzano il Manuale dovranno adattarlo alle loro specifiche esigenze.

Il tema generale del Manuale si basa sul fatto che, per quanto alcune problematiche siano complesse e molti aspetti debbano ancora essere chiariti (e potrebbero non essere mai risolti), molto è stato portato a termine e molto ancora può essere fatto nell'immediato da tutti gli attori 
coinvolti nella creazione e/o acquisizione di risorse digitali. Quest'attività aiuterà a proteggere l'iniziale investimento nella creazione di risorse digitali e ad offrire prospettive decisamente migliori nel lungo periodo.

\section{Principi guida}

I seguenti principi hanno guidato lo sviluppo del Manuale, il quale dovrebbe:

- Essere in linea con la missione della DPC di indipendenza dai fornitori di beni e servizi, incoraggiando gli utenti ad una partecipazione proattiva e spingendoli a condividere conoscenze e buone pratiche.

- Essere derivato dalle necessità della comunità di conservazione digitale ed essere capace di apportare loro vantaggi. La comunità di utenti reali e potenziali del Manuale verrà valutata e la sua composizione sarà usata per informare in maniera selettiva sulle successive evoluzioni.

- Essere aggiornato, attuale, conciso, e equilibrato. I capitoli forniranno delle panoramiche sintetiche sugli argomenti principali con una selezione di letture aggiuntive e casi di studio che offrono la possibilità di approfondire le tematiche con ulteriori dettagli. La selezione di letture aggiuntive e casi di studio sarà guidata dall'utilità, attualità e rilevanza del contenuto.

- Ridurre le barriere riferite alla partecipazione di attività di conservazione digitale con una strutturazione formalizzata, ma nello stesso tempo accessibile, del testo.

- Avere un contenuto autorevole e sottoposto a revisioni, con dei criteri di qualità espliciti come quelli stabiliti nelle Note per gli Autori, Revisori ed Editori della DPC, e nel piano progettuale del Manuale.

- Lavorare insieme e raggiungere delle sinergie con i report contenuti nelle serie TECH WATCH DPC. I report offrono "immersioni profonde" sia in specifiche aree contenutistiche della conservazione (ad esempio, e-mail) o argomenti principali (ad esempio, informatica forense) che possono essere citati o usati per navigare tra i casi studio del Manuale.

- Promuovere altre risorse (ad esempio, la Community Owned digital Preservation Tools registry - COPTR) che potrebbero essere gli strumenti più adatti a fornire informazioni specifiche oltre a quelle presenti nel Manuale.

- Promuovere altre risorse (ad esempio, report e guide) accessibili da web e gratuite per gli utenti.

- Essere sviluppato in maniera tale da facilitare il mantenimento, l'economicità e la sostenibilità nel lungo periodo da parte della DPC.

\section{Sviluppi futuri e supporto}

Al termine della seconda edizione, la DPC si prefigge lo scopo di integrare il suo aggiornamento e revisione con le sue serie di Digital Preservation Technology Watch Report. II controllo per l'avvio della revisione dei capitoli del Manuale e dei Report, o di nuovi topic per i Report o del contenuto del Manuale saranno responsabilità del comitato editoriale. 


\section{Come usare il Manuale}

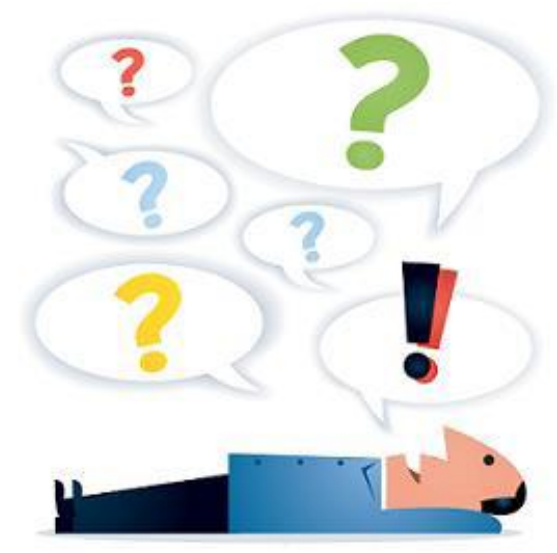

Illustrazione di Jørgen Stamp digitalbevaring.dk CC BY 2.5 Denmark

\section{Applicarlo nella pratica}

Le esigenze delle istituzioni in riferimento alle risorse digitali create e acquisite variano in modo decisivo. Questo Manuale ha lo scopo di fare da ponte tra il livello generale, le visioni d'insieme di alto livello, e le linee guida esplicite e dettagliate applicabili ai bisogni di un organismo specifico. Una visione strategica si collega alle attività operative al fine di potenziare la necessità di realizzare delle procedure basate sulle competenze istituzionali dell'organizzazione. II Manuale fornisce dei suggerimenti su dove poter trovare ulteriori guide e su come agevolare lo sviluppo di politiche e pratiche che siano applicabili al singolo caso di studio.

II Manuale dovrebbe idealmente essere usato per supportare gli utenti a orientarsi, per migliorare il livello generale di comprensione, per promuovere una formazione mirata e rappresentare una sorta di catalizzatore per azioni future. Ciò non toglie che ciascuna organizzazione possa allocare le risorse necessarie per attuare un piano d'azione, in ogni caso questo Manuale ha lo scopo di agevolare questo processo.

\section{Risorse del Manuale}

Questo Manuale promuove un approccio generale alla conservazione di risorse digitali basato su solidi principi e politiche condivise piuttosto che su formule prescrittive. Dal momento che l'importanza rivestita dalla conservazione digitale è sempre più forte, esistono diversi tipi di fonti affidabili di orientamento, e, nonostante il loro valore, la loro proliferazione rende più difficile il processo di selezione per una data circostanza.

Selezionando Risorse e Casi di studio in ogni sezione, il Manuale dovrebbe rendere più facile la navigazione tra le varie risorse e soluzioni esistenti utilizzando una combinazione di alberi decisionali, liste di controllo, campioni selezionati, casi di studio, e commenti. Questi hanno l'obiettivo di stimolare e promuovere riflessioni e discussioni approfondite, ma, soprattutto, di 
incoraggiare le istituzioni a sviluppare una gestione di politiche di conservazione digitale adatta ai loro bisogni.

Le risorse sono state raggruppate in categorie e identificate con le seguenti icone:

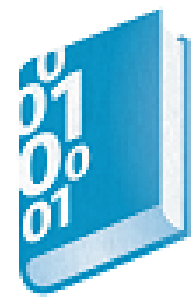

Pubblicazioni

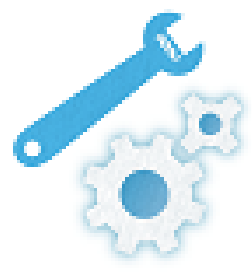

Strumenti

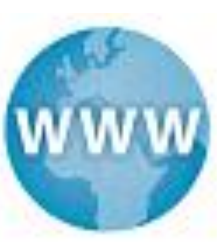

Risorse Web



Video e webinars

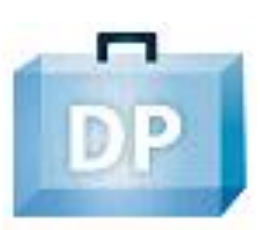

Casi di studio

\section{Prefazione generale}

II Manuale è destinato a un pubblico ampio e variegato, che va da utenti che stanno solo iniziando a considerare la gestione di risorse digitali ai professionisti che hanno già acquisito esperienze teoriche e/o pratiche. II Manuale è stato scritto con lo scopo di permettere un accesso veloce e facilitato alle sezioni d'interesse.

Ogni sezione è preceduta da una breve presentazione per aiutare ad individuare meglio il pubblico dei destinatari, il loro presupposto livello di conoscenze in relazione ai contenuti della sezione. La tabella qui di seguito aiuta a decidere quale sia la sezione più adatta per ciascuno. Non va, tuttavia, considerata come una scelta rigorosamente prescrittiva; infatti, chiunque può leggere il Manuale nella sua interezza.

Le Executive Lens, Manager Lens, e Practitioner Lens of the DigCurV Curriculum Framework for Digital Curation (DigCurV, 2013) sono state utilizzate all'interno del Manuale in generale come classificazioni per la profilazione degli utenti.

Consigliamo a tutti i lettori di leggere l'Introduzione.

II Glossario fornisce la spiegazione sintetica dei concetti chiave e le definizioni degli acronimi e delle iniziali usate dagli organizzatori e dai progetti all'interno del Manuale. 
Sezioni raccomandate e pubblici

\begin{tabular}{|l|l|}
\hline PUBBLICO & SEZIONI RACCOMANDATE \\
\hline $\begin{array}{l}\text { Tutti coloro che necessitano di } \\
\text { un'introduzione all'argomento }\end{array}$ & $\begin{array}{l}\text { Briefing sulla conservazione digitale } \\
\text { Guida introduttiva }\end{array}$ \\
\hline Creatori ed editori & $\begin{array}{l}\text { Briefing sulla conservazione digitale } \\
\text { Attività organizzative } \\
\text { Soluzioni tecniche e strumenti }\end{array}$ \\
\hline Agenzie di finanziamento & $\underline{\text { Briefing sulla conservazione digitale }}$ \\
\hline $\begin{array}{l}\text { Gestori operativi (DigCurV Manager } \\
\text { Lens) }\end{array}$ & $\underline{\text { Strategie istituzionali }}$ \\
$\underline{\text { Attività organizzative }}$ \\
$\underline{\text { Soluzioni tecniche e strumenti }}$ \\
\hline $\begin{array}{l}\text { Personale operativo (DigCurV } \\
\text { Practitioner Lens) }\end{array}$ & $\underline{\text { Guida introduttiva }}$ \\
$\underline{\text { Strategie istituzionali }}$ \\
$\underline{\text { Attività organizzative }}$ \\
$\underline{\text { Soluzioni tecniche e strumenti }}$ \\
Conservazione dei contenuti specifici \\
\hline $\begin{array}{l}\text { Amministratori senior (DigCurV } \\
\text { Executive Lens) }\end{array}$ & $\underline{\text { Strategie istituzionali }}$ \\
\hline Fornitori di servizi terzi & $\underline{\text { Strategie istituzionali }}$ \\
$\underline{\text { Attività organizzative }}$ \\
Soluzioni tecniche e strumenti \\
\hline
\end{tabular}

\section{Riutilizzo del Manuale}

\section{Riutilizzo in lingua inglese}

II manuale è disponibile in inglese sotto licenza Open Government v3.0 http://www.nationalarchives.gov.uk/doc/open-government-licence/version/3/, così è possibile utilizzarlo in maniera più ampia. Accettiamo di incorporare sezioni del Manuale nei manuali di formazione e in altre risorse. Si prega di usare questa formula di citazione di utilizzo del 
Manuale: Digital Preservation Handbook, 2nd Edition, https://www.dpconline.org/handbook Digital Preservation Coalition @ 2015 licensed under the Open Government Licence v3.0.

digitalbevaring.dk ha gentilmente dato il permesso di usare le illustrazioni della sezione dei titoli nel Manuale e le icone nelle Risorse e Casi di studio, prodotte da Jørgen Stamp, copyright di https://digitalbevaring.dk/ e condivise sotto licenza CC BY 2.5 Denmark (illustrazioni) https://creativecommons.org/licenses/by/2.5/dk/deed.en GB, e CC0 1.0 license (icone) https://creativecommons.org/share-your-work/public-domain/cc0.

Questi materiali si possono riutilizzare se le rispettive note di merito e ringraziamenti vengono inclusi nel Manuale.

Si prega tuttavia di tener conto che il Manuale continuerà ad essere aggiornato e supportare gli utenti:

1. Fornendo un link url associato all'ultima edizione online del Manuale sul sito della DPC così che gli utenti possano verificare gli ultimi aggiornamenti.

2. Unendo la lista di Conservazione Digitale su Jiscmail cosicché ognuno possa ricevere notifiche future sugli ultimi cambiamenti e aggiornamenti del Manuale. Per iscriversi alla lista cliccare su https://www.jiscmail.ac.uk/cgi-bin/webadmin?A0=DIGITALPRESERVATION

3. Citando l'utilizzo del Manuale.

Oltre ad essere buona prassi, può essere utile per la DPC al fine di aggiornare finanziatori e sponsor del Manuale e mostrare il suo impatto.

Traduzioni e riutilizzo in altre lingue

Accettiamo richieste di traduzione del Manuale in altre lingue. La DPC seguirà i principi e gli accordi presi per le traduzioni delle sue altre pubblicazioni e ne pianificherà uno formale per la traduzione così che tutti gli accordi risultino chiari per un lungo periodo di tempo. In particolare vorremmo:

- Caricare la traduzione sul sito web della DPC. L'uso che viene fatto delle nostre pubblicazioni è una delle nostre principali misurazioni d'impatto, perciò c'è l'esigenza di assicurare la disponibilità di conseguenti analitiche web.

- Assegnare un DOI alla traduzione. Questo significa che gli utenti potrebbero risalire al report sia dal sito della DPC che da altre pagine: altre organizzazioni potrebbero associargli qualsiasi pagina sorgente. Significa anche che ci impegneremmo a mantenere l'url in forma permanente (cioè il traduttore non ha bisogno di farlo).

- Mantenere il marchio e i crediti presenti nella pubblicazione. Proporremmo una premessa speciale o un'introduzione che potrebbe includere una descrizione della traduzione e saremmo propensi a un logo aggiuntivo e marchio che possano essere evidenti nella pagina principale e nel Manuale.

- Mantenere l'ultima parola sul risultato finale dal momento che la DPC ha un ruolo importante nella decisione di qualità e reputazione della versione tradotta del Manuale. 
Ciò potrebbe comportare l'invio di questa traduzione a un lettore nativo per una revisione indipendente.

\section{Riferimenti}

DigCurV, 2013. A Curriculum Framework for Digital Curation. Disponibile al seguente link: https://www.digcurv.gla.ac.uk/ 


\section{Creazione e ringraziamenti}

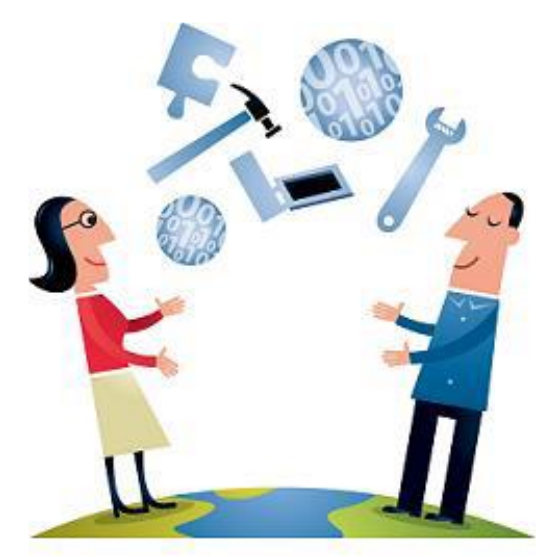

Illustrazione di Jørgen Stamp digitalbevaring.dk CC BY 2.5 Denmark

\section{Creazione del Manuale}

II Manuale è stato pubblicato per la prima volta nel 2001 come edizione stampata, Preservation Management of Digital Materials: a Handbook, stampato dalla British Library Publishing (Jones and Beagrie, 2001). II CD in edizione limitata del Manuale è stato realizzato nel Febbraio 2002 per il lancio della DPC presso la House of Commons. La versione digitale online del Manuale è stata resa disponibile sul sito web della DPC subito dopo, a Maggio del 2002. Una versione archiviata di questa "prima edizione online" del Manuale è disponibile nell'Archivio Web UK (UKWA, 2009).

Per oltre un decennio, la versione online del Manuale ha rappresentato una guida autorevole e pratica a livello internazionale per quanto riguarda la gestione di risorse digitali nel tempo e gli aspetti legati a garantirne l'accesso. Tuttavia, dal momento in cui è stato reso accessibile online, è stato apportato solo un numero ridotto di aggiunte al Manuale. La DPC stabilì un target strategico per incrementare finanziamenti di ricerca al fine di aggiornare in forma completa il Manuale e integrarlo in modo conforme alle serie Technology Watch allo scopo di mantenere inalterate le sue caratteristiche.

Nel 2014, il National Archives (TNA), in qualità di finanziatore leader, accanto a finanziamenti supplementari da Archives and Records Association, British Library, JISC, e National Archives of Scotland, offrì un finanziamento di ricerca per iniziare la revisione integrale del Manuale e promuoverne l'utilizzo. Questo lavoro fu intrapreso sotto la direzione di William Kilbride, Direttore di DPC, e dall'editore Neil Beagrie, e supportato da un ampio comitato consultivo e da un gruppo di collaboratori. 
La Digital Preservation Coalition (DPC) è un'organizzazione non-profit che rende il Manuale di Conservazione Digitale gratuitamente accessibile come risorsa online: è ampiamente utilizzato per lo Sviluppo professionale continuativo, da studenti universitari, e per la formazione in conservazione digitale. Una revisione della formazione in conservazione digitale del progetto APARSEN ha evidenziato un gruppo ristretto di argomenti trattati da più iniziative (APARSEN, 2012). Sottolinea divari concreti nella copertura di argomenti che suggerivano come un'edizione revisionata aggiornata del Manuale potesse andare incontro ad esigenze attuali.

Parte integrante della preparazione alla seconda edizione è il mapping che venne avviato tra le raccomandazioni ARPASEN e il Manuale, e anche al DigCurV Curriculum Framework for Digital Curation (DigCurV, 2013). Un'ampia indagine di ricerca rese noti gli sviluppi proposti per il Manuale che inclusi suggerimenti provenienti da 285 membri della comunità attraverso un'indagine online (DPC, 2014a) e una consultazione pubblica sulla bozza di schema del contenuto (DPC, 2014b).

Per i riferimenti è stato usato il sistema Harvard, seguendo lo stesso stile dei DPC Technology Watch Reports.

L'interesse a coinvolgere la comunità di conservazione digitale a rendere accessibile il Manuale riflette i bisogni propri di un ampio gruppo di organizzazioni e settori, ed è rimasto attivo durante tutta la sua revisione attraverso l'utilizzo di "book sprint"18 collaborativi e il suo comitato consultivo.

\section{Ringraziamenti}

La seconda edizione del Manuale di Conservazione Digitale è stata realizzata all'interno di un progetto di ricerca collaborativo basato su un accordo di consorzio tra National Archives, The Archives and Records Association, la British Library, Charles Beagrie Ltd, la Digital Preservation Coalition, e JISC.

- Il finanziamento del progetto venne avviato dal National Archives. Consapevole della sfida della conservazione digitale affrontata dai settori di archivistica, e attivo nel suo compito di fornire una gestione strategica di settore, ha offerto il $50 \%$ del capitale previsto richiesto (ovvero Gold Sponsorship) nella prospettiva in cui la DPC avrebbe sia ricercato finanziamenti rimanenti attirando altri sponsor sia coperto direttamente i costi.

- II comitato DPC, che voleva revisionare il Manuale, ha accettato l'offerta del National Archives, che dava tempi di lavoro e assicurava che i costi delle risorse (come ad esempio il sito web) potessero essere gestiti senza spese. La DPC ha anche donato un contributo monetario da finanziamenti interni.

\footnotetext{
18 Un metodo per creare un libro in modo collaborativo in un breve periodo di tempo.
} 
- Charles Beagrie Ltd è entrato a far parte del consorzio come partner editoriale per avviare una ricerca originale, sviluppare contenuti e progetti, e coordinarne la garanzia di qualità. Charles Beagrie Ltd ha contributo in termini di tempi di lavoro (equivalente al Bronze Sponsorship), aiutando nelle attività editoriali finali per il completamento del Manuale.

- JISC si è unito alla collaborazione fin dall'inizio riconoscendo che gli archivisti e i gestori di dati della ricerca nelle istituzioni accademiche superiori avrebbero apprezzato l'utilizzo del Manuale. I finanziamenti rilasciati da JISC (Silver Sponsorship) hanno permesso lo sviluppo delle tre sezioni specifiche di conservazione e della metodologia "book sprint".

- La British Library sì è unita alla collaborazione sin dall'inizio, ha avuto un ruolo fondamentale nella versione originale del Manuale. I fondi della British Library (Silver Sponsorship) hanno permesso un accertamento delle esigenze complessive e delle indagini, e ha costituito la base per l'indice revisionato.

- In seguito a un periodo di sviluppo del contenuto e della creazione di prototipi, la Archives and Records Association (ARA) ha preso parte alla collaborazione offrendo dei finanziamenti (Silver Sponsorship) da capitali di ricerca personali, garantendo che i testi di bozza fossero sistematicamente e interamente revisionati prima della loro pubblicazione, e che la DPC fosse in grado diffondere l'uso del Manuale in una serie di eventi di formazione accessibili ai membri ARA.

- Infine, i finanziamenti sono stati avviati dal National Records of Scotland (Bronze Sponsorship) per permettere una diffusione più ad ampio raggio e un lancio immediato.

II comitato DPC ringrazia formalmente i partner, soprattutto per i diversi contributi supplementari, introduzioni e incoraggiamenti che hanno portato al completamento del progetto.

Finanziatori

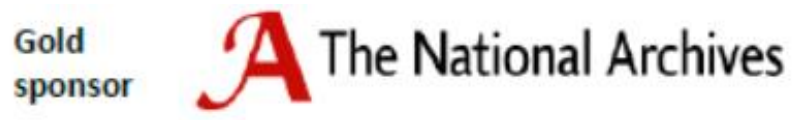
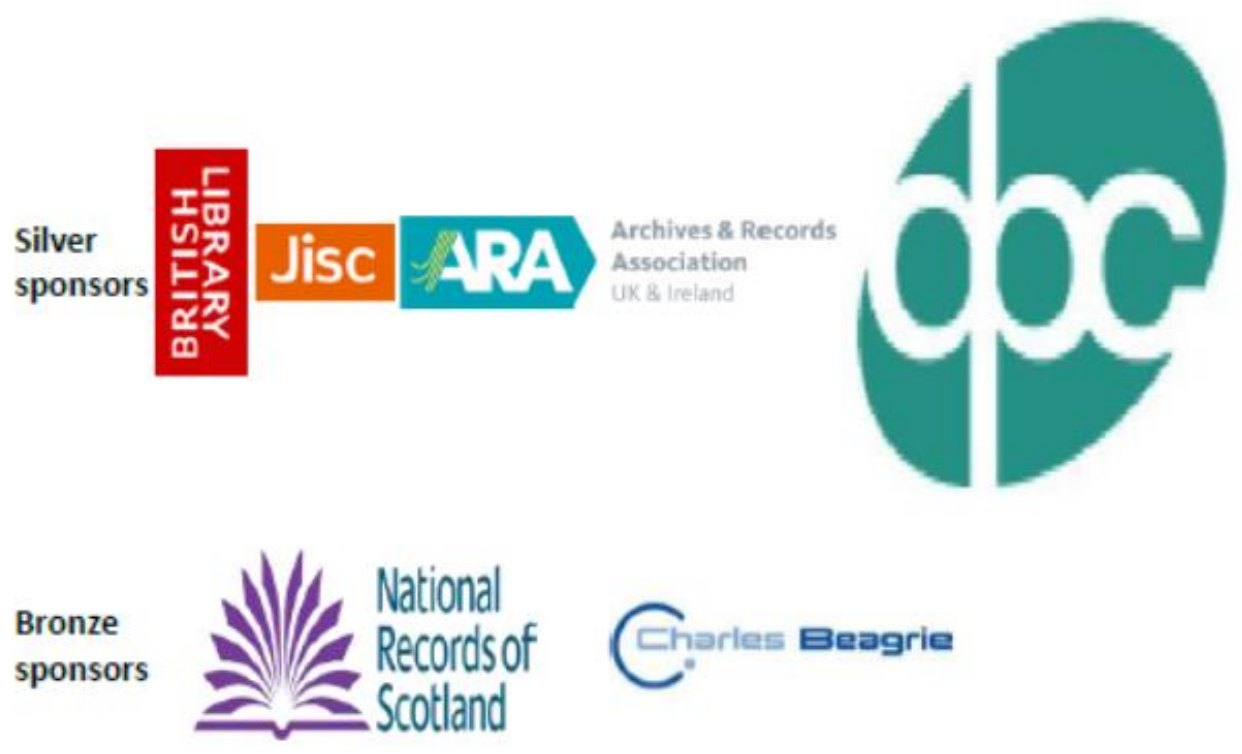
digitalbevaring.dk ha gentilmente dato i permessi per utilizzare le illustrazioni presenti nella sezione dei titoli del Manuale e le icone presenti nella sezione Risorse e Casi di studio, prodotte da by Jørgen Stamp, copyright di digitalbevaring.dk e condivise sotto licenza CC BY 2.5 Denmark licence (illustrazioni) https://creativecommons.org/licenses/by/2.5/dk/deed.en GB, e licenza CC0 1.0 (icone) https://creativecommons.org/share-your-work/public-domain/cc0.

\section{Direttore di redazione del Manuale: Sharon McMeekin (da giugno 2016)}

\section{Ex Direttore di Redazione: Neil Beagrie (fino a giugno 2016)}

Collaboratori del Manuale:

\begin{tabular}{|c|c|}
\hline Matthew Addis & Arkivum Ltd (book sprint 1) \\
\hline Neil Beagrie & $\begin{array}{l}\text { Charles Beagrie Ltd (book sprint } 1 \text {, book sprint } 2 \text {, book sprint } 3 \text {, } \\
\text { book sprint } 4 \text {, servizi Cloud, Glossario, } 1^{\text {a }} \text { edizione del Manuale, } \\
\text { Introduzione, casi di studio sulla conservazione delle riviste } \\
\text { elettroniche, Risorse e casi di studio) }\end{array}$ \\
\hline Daphne Charles & $\begin{array}{l}\text { Charles Beagrie Ltd (Progettazione del contenuto web e input } \\
\text { del Manuale, ricerche documentali e sul pubblico per il Manuale) }\end{array}$ \\
\hline Andrew Charlesworth & University of Bristol (servizi Cloud) \\
\hline Tracey Clarke & University of Sheffield (book sprint 3) \\
\hline Glenn Cumiskey & British Museum (book sprint 2) \\
\hline Stefanie Davidson & West Yorkshire Archive Service (book sprint 1) \\
\hline Michael Day & British Library (book sprint 1) \\
\hline Matt Faber & JISC (book sprint 1, book sprint 2) \\
\hline Chris Fryer & Parliamentary Archives (book sprint 1) \\
\hline Dave Govier & Manchester Met Archives Network (book sprint 3) \\
\hline Stephen Grace & University of East London (book sprint 2) \\
\hline Alex Green & The National Archives (book sprint 2) \\
\hline Edith Halvarsson & British Library (book sprint 3) \\
\hline Anna Henry & Tate Gallery (book sprint 1 ) \\
\hline
\end{tabular}




\begin{tabular}{|l|l|}
\hline Sarah Higgins & University of Aberystwyth (book sprint 3) \\
\hline Jeremy Leighton John & British Library (Informatica forense) \\
\hline Maggie Jones & $\begin{array}{l}\text { precedentemente Digital Preservation Coalition (1a edizione del } \\
\text { Manuale) }\end{array}$ \\
\hline William Kilbride & $\begin{array}{l}\text { Digital Preservation Coalition (book sprint 1, book sprint 3, book } \\
\text { sprint 4) }\end{array}$ \\
\hline Gareth Knight & London School of Hygiene and Tropical Medicine (book sprint 2) \\
\hline Sharon McMeekin & $\begin{array}{l}\text { Digital Preservation Coalition (book sprint 2, book sprint 3, book } \\
\text { sprint 4) }\end{array}$ \\
\hline Paul Miller & Cloud of Data (servizi Cloud) \\
\hline Jenny Mitcham & University of York (book sprint 3) \\
\hline Laura Peaurt & University of Sheffield (book sprint 3) \\
\hline Maureen Pennock & British Library (Archiviazione Web) \\
\hline Ed Pinsent & University of London Computer Centre (book sprint 1) \\
\hline Virginia Power & JISC (book sprint 1) \\
\hline Vicky Stretch & Network Rail (book sprint 3) \\
\hline Susan Thomas & Bodleian Library University of Oxford (book sprint 1) \\
\hline Dave Thompson & Wellcome Trust (book sprint 3) \\
\hline Paul Wheatley & $\begin{array}{l}\text { Digital Preservation Coalition (book sprint 2, book sprint 3, book } \\
\text { sprint 4) }\end{array}$ \\
\hline Simon Wilson & Hull University Archives (book sprint 3) \\
\hline Richard Wright & formerly BBC (Immagini in movimento e suono) \\
\hline
\end{tabular}


Consiglio di amministrazione

\begin{tabular}{|l|l|}
\hline CHAIR: William Kilbride & Digital Preservation Coalition \\
\hline John Chambers, Chris Fryer & Archives and Records Association \\
\hline Maureen Pennock & British Library \\
\hline Neil Beagrie (editor) & Charles Beagrie Ltd \\
\hline Neil Grindley, Virginia Power, Matt Faber & JISC \\
\hline $\begin{array}{l}\text { Emma Markiewicz, Matt Greenhall, Jane Anderson, } \\
\text { Isobel Hunter }\end{array}$ & The National Archives \\
\hline
\end{tabular}

\section{Comitato consultivo}

\begin{tabular}{|l|l|}
\hline CHAIR: William Kilbride & Digital Preservation Coalition \\
\hline Nancy Y McGovern & MIT Libraries \\
\hline Marcel Ras & NCDD, Dutch National Coalition for Digital Preservation \\
\hline Timothy Gollins & The National Archives and National Records of Scotland \\
\hline Stefanie Davidson & West Yorkshire Archive Service \\
\hline Sarah Higgins & Department of Information Studies, Aberystwyth University \\
\hline Joy Davidson & Digital Curation Centre, University of Glasgow \\
\hline Virginia Power \& Matt Faber & JISC \\
\hline Anna Henry & Tate Gallery \\
\hline Chris Fryer & Parliamentary Archives \\
\hline Simon Tanner & King's College London \\
\hline Michael Day & British Library \\
\hline Susan Thomas & Bodleian Library University of Oxford \\
\hline Carla Shields & Public Record Office of Northern Ireland \\
\hline Marion Downie & The National Archives \\
\hline Hania Smerecka & Lloyds Banking Group Archives \\
\hline
\end{tabular}




\section{Revisori}

Ringraziamo inoltre le 26 persone che hanno contribuito alle revisioni delle sezioni del Manuale pertinenti alle loro aree di interesse e di competenza. Ringraziamo anche i membri del comitato consultivo e Hugh Campbell (Public Record of Northern Ireland), Andrew Charlesworth (University of Bristol), Carey Clifford (Grosvenor Group), Lee Hibberd (National Library of Scotland), Neil Jefferies (University of Oxford), Catherine Jones (Science and Technology Facilities Council), Naomi Korn (Naomi Korn Copyright Consultancy Ltd ), Ingrid McDonald (Queensland State Archives), Laura Molloy (University of Oxford), Barbara Sierman (KB), Charlene Taylor (Worcestershire Archive \& Archaeology Service), Kate Watson (Dorset County Council), Rebecca Webster (UCL Institute of Education), e Cathy Williams (The National Archives) per le loro revisioni e suggerimenti che hanno portato contributi significativi al Manuale.

\section{Riferimenti}

APARSEN, 2012. D43.1 Survey for the Asssessment of Training Material/Assessment of Digital Curation Requirements. Accessibile a: https://www.dpconline.org/docs/knowledge-base/18172012-02-21-aparsen-d43-1/file

DigCurV, 2013. A Curriculum Framework for Digital Curation. Disponibile al seguente link: https://www.digcurv.gla.ac.uk/

DPC, 2014a. Report on the Preparatory User Consultation on the 2nd Edition of the Digital Preservation Handbook. Accessibile a: https://www.dpconline.org/docs/miscellaneous/advice/1251-handbook-survey-responsesummary-redacted/file

DPC, 2014b. Draft Outline of the 2nd Edition of the Digital Preservation Handbook. Accessibile a: https://www.dpconline.org/docs/miscellaneous/advice/1306-handbook-new-contents/file Jones, M. \& Beagrie, N., 2001. Preservation Management of Digital Materials, A Handbook. The British Library

UKWA (UK Web Archive), 2009. 8 captures (April 2008-December 2009) of the first edition of the online version of the Handbook. Accessibile a: https://www.webarchive.org.uk/wayback/archive/20090317142605/http://www.dpconline.org/gr aphics/handbook/ 


\section{Briefing sulla conservazione digitale}

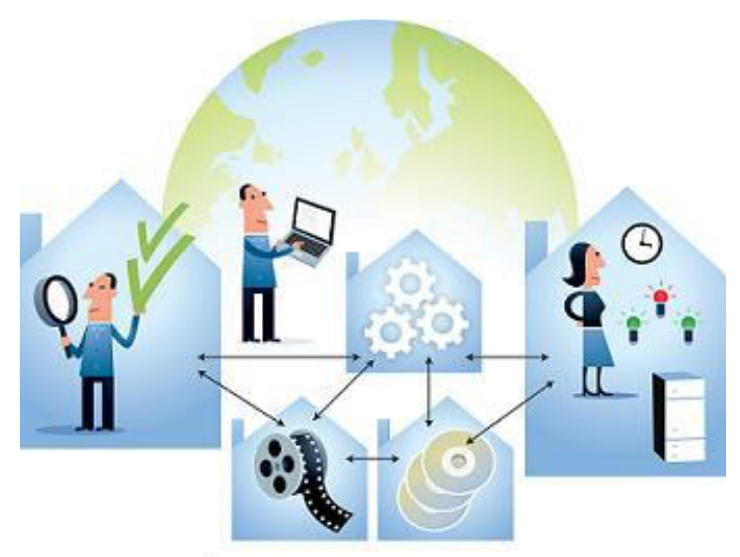

Illustrazione di Jørgen Stamp digitalbevaring.dk CC BY 2.5 Denmark

\section{A chi è rivolto?}

Responsabili senior (DigCurV Executive Lens), direttori operativi (DigCurV Manager Lens) e staff (DigCurV Practitioner Lens) all'interno di archivi, agenzie di finanziamenti, creatori e editori, a chiunque abbia bisogno di un'introduzione all'argomento.

Requisiti minimi di conoscenza

Livello principiante.

\section{Obiettivo}

- Fornire una panoramica strategica e una sintesi sulla gestione dirigenziale, descrivendo le problematiche generali e la logica per cui i finanziamenti siano destinati alle attività coinvolte nella conservazione di risorse digitali;

- Portare in evidenza discussioni attuali sulle questioni legate alla conservazione digitale;

- Distinguere tra le principali categorie di questioni;

- Aiutare a chiarire come le varie questioni impatteranno sulle decisioni ai vari livelli del ciclo di vita delle risorse digitali;

- Dare uno spunto di riflessione per futuri dibattiti e discussioni all'interno di organizzazioni e con pubblici esterni. 


\section{Perché è importante la conservazione digitale}



Illustrazione di Jørgen Stamp digitalbevaring.dk CC BY 2.5 Denmark

\section{Introduzione}

Questa sezione, insieme alla sezione Conservazione, è stata progettata con l'idea di fornire delle linee guida a coloro che si approcciano per la prima volta al tema della conservazione digitale. Si presenta suddivisa in quattro sottosezioni interrelate. Inoltre, ha una stretta corrispondenza con la sezione Guida introduttiva, anch'essa ideata per chi si approccia alla conservazione digitale.

\section{Conservazione digitale: la sfida generazionale}

Ogni oggetto digitale può essere considerato come un'estensione della conservazione digitale: che sia nato digitale o digitalizzato, imprenditoriale o privato, innovativo o ordinario. La conservazione digitale può riguardare testi e immagini, database e fogli di calcolo, vettori o raster $^{19}$, programmi e applicazioni, file desktop e sistemi d'impresa, e-mail e social media, giochi, film, musica e suono, interi domini web e tweet personali. Le collezioni digitali possono provenire dai laptop, dai desktop o dagli smartphones; dai tablet, da server truccati o sistemi centrali di grandi dimensioni. possono venire scattate con un bastone da selfie o trasmesse da sensori nello spazio; possono essere generate da casse e sportelli automatici, da satelliti e scanner; da piccolissimi chip fotosensibili o da grandi vettori. Possono essere conservate in archivi $\mathrm{o}$ in data center o su supporti USB. Non c'è oggetto digitale o sistema che non rientri temporaneamente nell'ambito della conservazione digitale.

\footnotetext{
19 La grafica raster, o bitmap, è una tecnica usata per descrivere un'immagine in formato digitale che si contrappone alla grafica vettoriale. L'immagine è composta da una griglia di punti detti pixel, di forma quadrata.
} 
Le tecnologie digitali, pervasive, mutevoli e onnipresenti, sono un tratto distintivo del nostro tempo. Le risorse digitali sono un bene fondamentale per le industrie, il commercio e lo Stato. Sono fondamentali per la ricerca, l'ambito legale e sanitario. Le industrie creative, il patrimonio culturale e i media dipendono dall'accesso sicuro alle risorse digitali, e le famiglie e cerchie di amici estendono e sostengono le loro relazioni attraverso interazioni digitali.

Ma le risorse digitali - e le opportunità che esse creano - sono fragili sebbene abbiano la capacità di essere durature nella loro riproducibilità. I portali digitali cambiano e le grandi catene di interdipendenza sulle quali si basano sono complesse e fluide. La loro longevità e utilità sono minacciate nel momento in cui i contenuti o i contesti vengono perduti: l'impiego e l'utilizzo vengono consentiti nel momento in cui si conservano le risorse digitali. Più aumenta l'importanza data alle risorse digitali, più cresce il bisogno della loro conservazione: la conservazione digitale protegge l'investimento, capta il potenziale e comunica le opportunità alle nuove generazioni e a quelle attuali.

Abbiamo già fatto passi da gigante nello scongiurare "l'età digitale oscura". C'è un numero crescente di archivi in tutto il mondo che può vantare una lunga esperienza nel buon mantenimento delle risorse digitali per diversi decenni (per esempio l'archivio inglese Data Archive fondato nel 1967). Ciò ci garantisce una buona base generale d'esperienza e reti professionali collaborative a cui attingere.

È una sfida generazionale condivisa.

\section{La sempre costante sfida della conservazione digitale}

II tratto comune tra le risorse digitali è il loro essere dipendenti dall'informatica. Infatti, si può accedere alle informazioni e le funzioni possono essere eseguite solo attraverso un computer. Negli anni la tecnologia è diventata più sofisticata, perciò anche questa dipendenza diviene una catena ancora più complessa di interdipendenze che sono difficilmente tracciabili e non semplici da mantenere.

Fin quando il settore IT continua ad essere innovativo nelle offerte di nuovi strumenti e tecnologie, la conservazione digitale risponderà predisponendo delle strategie efficaci che possano assicurare la durabilità e l'utilizzo delle risorse digitali. In questo modo, la conservazione digitale rimarrà una costante sfida.

Al fine di assicurare il valore delle risorse digitali nel lungo periodo dobbiamo assicurarne l'accesso, il che, a sua volta, significa che dobbiamo comprendere e mitigare i rapidi cambiamenti che avvengono nel campo della tecnologia e tra le organizzazioni (si veda La Conservazione).

Ad oggi disponiamo di una gamma di approcci, esperienze e collaborazioni emergenti ed efficaci da poter impiegare per questa sfida. La conservazione digitale è importante, necessaria è uno sforzo fattibile che ciascuno, a piccoli passi, può affrontare (si veda Guida introduttiva). 


\section{Qual è l'ambito?}

Solo perché qualsiasi cosa potrebbe rientrare in un piano strategico di conservazione digitale, non significa che tutto debba essere conservato.

La domanda da porsi non è tanto ciò che può essere conservato, quanto ciò che non dovrebbe andar perso. La selezione, la valutazione e l'eliminazione sono elementi importanti in ogni attività di conservazione digitale. Nella prospettiva di un'espansione digitale, un determinato sforzo nell'identificare, processare e conservare una risorsa digitale di valore duraturo significa, da una parte, che la risorsa corretta è accessibile da parte degli utenti autorizzati nel tempo corretto e nel formato corretto; dall'altra, che la risorsa identificata può essere totalmente rimossa o ignorata.

Una risorsa digitale offre nuove e importanti opportunità d'accesso e di uso degli archivi. Dal momento che le collezioni digitali si trovano in un ambiente in rapido cambiamento, ci dovremmo aspettare che anche i nostri utenti lo siano. Gli utenti delle risorse digitali solitamente utilizzano una tecnologia ancora non pienamente sviluppata in modi che non siamo in grado di anticipare completamente, in luoghi che potremmo non visitare mai e per scopi che fatichiamo a predire. Perciò, qualsiasi risposta significativa alla domanda "come possiamo conservare le risorse digitali" può essere riportata a "cosa possiamo fare per far sì che queste risorse digitali possano essere utilizzate"? Pianificare la conservazione avrà successo solo quando le esigenze degli utenti verranno soddisfatte.

Tutto ciò porta alla considerazione che, ove possibile, la conservazione nel lungo periodo delle risorse digitali dovrebbe essere definita prima e non dopo. L'azione di conservazione è necessaria sin dalla fase iniziale del ciclo di vita dell'oggetto digitale, non alla fine. La creazione, la gestione e l'archiviazione delle risorse digitali non si trovano più agli opposti di un processo, ma vengono integrati per tutta la sua durata. Di conseguenza, la conservazione non è più semplicemente un compito per le istituzioni di conservazione della memoria nel lungo periodo, ma per chiunque sia interessato all'uso e all'accesso delle risorse digitali.

\section{Chi deve essere coinvolto?}

La capacità di conservare le risorse digitali dipende da un'ampia gamma di stakeholder. Tra di loro i principali sono i creatori di contenuti digitali il cui coinvolgimento nella conservazione potrebbe comprendere, ad esempio, l'indicazione di standard riguardo ai formati e i media, nonché la capacità di garantire che le informazioni contestuali siano accessibili indipendentemente dal gestore. Spesso gli stakeholder possono essere inconsapevoli del loro ruolo fondamentale, e ciò può dipendere da moltissimi fattori, ma il dialogo effettivo con i creatori delle risorse digitali è parte vitale di qualsiasi attività di conservazione digitale.

Così come i creatori delle risorse digitali hanno la responsabilità di garantire l'accesso nel lungo periodo, anche chi fornisce le infrastrutture e gli ambienti in cui queste risorse sono create gioca un ruolo principale. In alcuni casi questo processo può essere coordinato dall'interno attraverso la fornitura di strumenti e servizi aziendali che sono pronti per l'attività di conservazione. In altri 
casi la responsabilità è sostenuta da fornitori di servizi esterni che mettono a disposizione le infrastrutture digitali per i clienti.

È la natura stessa della tecnologia digitale a dettare l'impraticabilità di cedere, ad un certo punto, la gestione di una risorsa senza averla gestita in modo sufficiente per favorirne la sostenibilità.

In alcuni casi, le istituzioni gestiranno la loro eredità digitale personalmente: le grandi organizzazioni che creano risorse digitali potrebbero essere quelle che le gestiranno nel lungo periodo in modo responsabile, così da massimizzare la redditività in rapporto al loro investimento iniziale. Tuttavia, in altri contesti i modelli cooperativi per la conservazione nel lungo periodo hanno visto coinvolte diverse organizzazioni. Sia esperti che centri specializzati hanno cominciato a offrire soluzioni di conservazione mirate per specifiche tipologie di risorse digitali.

Per alcune organizzazioni esternalizzare tutto o parte delle loro attività di conservazione a parti terze potrebbe avere un migliore rapporto costo-efficacia. Ma anche se esternalizzare può portare dei vantaggi, è comunque importante ricordare che la responsabilità rimane alle organizzazioni. Per gestire questi contratti con terze parti in maniera efficace, lo staff dovrà essere preparato sulle dinamiche di conservazione, in particolare quelle relative ai problemi legali, organizzativi e contrattuali.

Qualsiasi ente che valorizza le risorse digitali, in genere, ha bisogno di garantire la loro conservazione nel lungo periodo. Vi sono molte istituzioni che non soltanto se ne sono occupate personalmente, ma che hanno anche offerto una direzione più ad ampio raggio anche in relazione alle ripercussioni pratiche della conservazione digitale.

In ultima analisi, tuttavia, la conservazione digitale non può essere considerata una questione solo relativa agli archivi, biblioteche, musei e altri istituti di conservazione della memoria; si tratta invece di una sfida per chiunque abbia interesse a creare, usare, acquisire, e rendere accessibili le risorse digitali.

\section{Risorse}

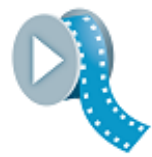

\section{Perché la conservazione digitale è importante per tutti}

https://www.youtube.com/watch?v=qEmmeFFafUs\&index=43\&list=PLEA69BE43AA9F7E68

Un breve video della Library of Congress prodotto nel 2010 per un pubblico di non esperti spiega come le tradizionali fonti d'informazione (libri, foto, sculture) possano sopravvivere con facilità negli anni, decenni e persino secoli; al contrario, gli oggetti digitali sono fragili e necessitano di cure particolari affinché possano essere riutilizzati. Anche i rapidi cambiamenti nella tecnologia 
giocano un ruolo importante nella conservazione digitale: quando ne sopraggiunge una nuova le altre divengono obsolete, rendendo difficile l'accesso a vecchi contenuti (2 minuti 51 secondi). 


\section{La conservazione}

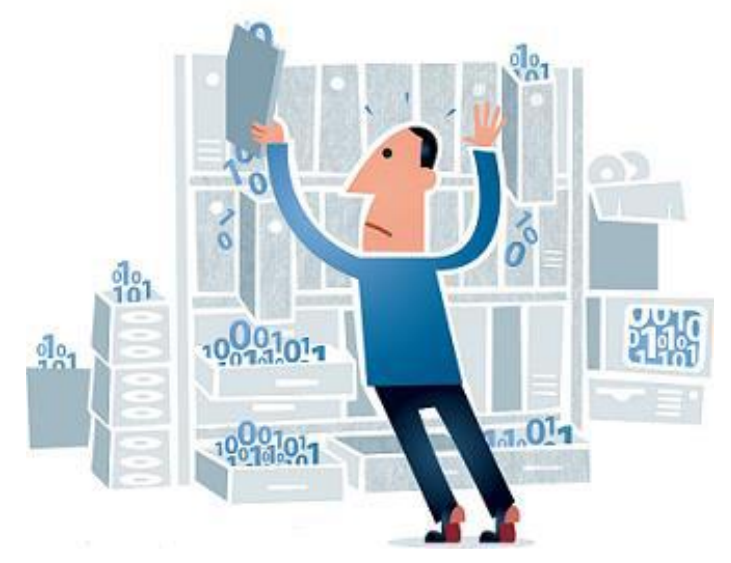

Illustrazione di Jørgen Stamp digitalbevaring.dk CC BY 2.5 Denmark

\section{Introduzione}

Questa sezione, insieme a Perché è importante la conservazione digitale, rappresenta un'introduzione per coloro che si approcciano per la prima volta al tema della conservazione digitale. Ė strutturata in tre sottosezioni collegate tra loro che descrivono le Minacce alle risorse digitali, gli aspetti Organizzativi, e gli aspetti legati alle Risorse. Si collega in modo più dettagliato ad altre sezioni all'interno del Manuale, in particolare alla sezione Guida introduttiva dal momento che anch'essa è progettata specificatamente per chi intende approcciarsi alla conservazione digitale.

All'inizio la conservazione digitale appare spesso scoraggiante. È importante comprendere, perciò, che chi ha già delle competenze sia nella gestione delle informazioni o tecnologie informative all'interno di organizzazioni si trova in una posizione ottimale per lavorare e applicare queste competenze alle attività di conservazione digitale. Tuttavia, potrebbe essere inizialmente necessario apprendere una nuova, e non familiare, terminologia (si veda Glossario), ampliare le proprie competenze e, a volte, lavorare secondo nuove modalità. 


\section{Minacce alle risorse digitali}

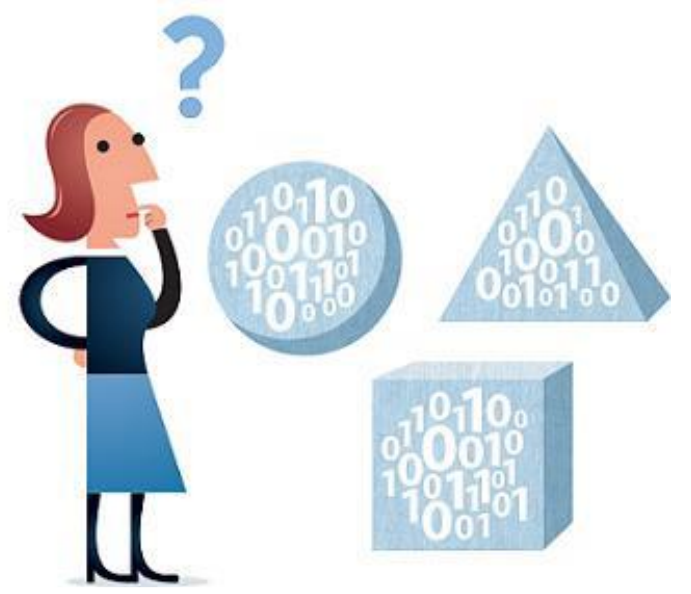

Illustrazione di Jørgen Stamp digitalbevaring.dk CC BY 2.5 Denmark

\section{Mantenere i dati}

Ogni file digitale è composto da una serie di zeri e uno, o bit (cifra binaria). Questi flussi di bit devono essere acquisiti e mantenuti nel tempo senza perdita o danno al fine di assicurare la sopravvivenza delle risorse digitali. Per ogni tentativo di conservazione di questi bit si presentano molteplici minacce. I supporti di archiviazione possono diventare desueti, portando ad avere file danneggiati. Possono diventare obsoleti e non supportati dai computer contemporanei e dai software che interpretano e consentono l'accesso. I bit possono venire ignorati, abbandonati, accidentalmente cancellati o intenzionalmente danneggiati. I supporti rimovibili potrebbero essere lasciati su uno scaffale e dimenticati, i file conservati su un'unità di rete condivisa potrebbero essere lasciati senza proprietario, oppure i fornitori di parti terze di archiviazioni cloud potrebbero cessare l'attività.

Mantenere un processo sistematico per la conservazione di bit rimane un requisito fondamentale per assicurare la conservazione digitale nel lungo periodo. I media con supporto devono essere controllati e aggiornati (si veda Supporti obsoleti). La ridondanza deve essere introdotta attraverso la replicazione o backup dei file, descrivendo differenze nelle tecnologie dipendenti ed evitando il verificarsi di eventi catastrofici su una singola postazione geografica (si veda Archiviazione). I checksum devono essere generati e ricalcolati con frequenza per identificare ogni perdita e assicurare la verifica dell'integrità dei bit in un modo efficiente e automatizzato (si veda Fixity e checksum). I punti in cui le risorse digitali vengono archiviate dovrebbero essere registrati con attenzione e dovrebbe essere assegnata la responsabilità per la loro conservazione. 


\section{Mantenere il significato dei dati}

Generalmente, la ricostruzione delle informazioni codificate entro un flusso di bit richiede dei software progettati per rappresentare, manipolare, analizzare, o, altrimenti, interagire con una particolare codifica o formato dei dati. Le codifiche (o i formati dei file) possono subire dei cambiamenti nel corso del tempo, e le applicazioni software che trattano questi ultimi possono essere ancora valide o passare di moda. Sebbene sia insolito per i formati dei file noti, quelli meno noti possono diventare obsoleti nel tempo perché i software che li interpretano non sono più supportati (si veda Formati dei file e standard).

Riuscire a comprendere la tecnologia dalla quale specifiche risorse digitali dipendono permette di intraprendere azioni appropriate per assicurare la loro conservazione. Un processo di pianificazione mirato di conservazione è quanto mai opportuno nella migrazione dei file digitali da formato a formato, nell'emulazione di software obsoleti o nell'utilizzo di applicazioni software alternative per interpretare i dati (si veda Azione di conservazione). Ognuna delle opzioni presenta i suoi vantaggi e svantaggi, e questi devono essere attentamente esaminati, possibilmente caso per caso (si veda Piano di conservazione).

Anche se l'obsolescenza dei formati dei file non viene considerata come un pericolo significativo, a differenza di quanto accadeva qualche anno fa, rimangono alcune insidie complesse. Si potrebbe trovare una metodologia per interpretare un vecchio formato di file (magari emulando qualche software obsoleto), ma quanto accurata è l'interpretazione? è consentito utilizzare il software? e quanto questo sforzo così complesso verrà a costare al conservatore e all'utente?

\section{Mantenere la fiducia nei dati}

Le risorse digitali hanno la caratteristica di rimanere fluide nel tempo, di essere modificate o alterate con facilità venendo danneggiate per deterioramento dei supporti, o decodificate in un'informazione leggibile per l'uomo in modo non affidabile o non accurato. Perché un utente finale abbia fiducia nel successo del risultato della conservazione digitale c'è bisogno di un'attenzione particolare all'intero ciclo di vita delle risorse digitali e a chi o cosa ha interagito con esse nel tempo. I sistemi di gestione dell'informazione devono essere in grado di collegare le informazioni contestuali essenziali relative alle procedure aziendali dell'ente produttore. L'autenticità e l'integrità delle risorse digitali sono parimenti importanti in altri settori. I ricercatori, ad esempio, avranno bisogno di essere sicuri che i riferimenti che citano rimangano invariati nel tempo, i tribunali avranno bisogno di essere certi che il materiale possa far fronte a richieste di evidenza giuridiche, gli uffici pubblici, allo stesso modo, potrebbero avere richieste relative all'autenticità, e via dicendo. La questione fa coincidere sia aspetti legali che organizzativi e potrebbe essere risolta al meglio all'interno di specifici settori anziché attraverso procedure generiche.

L'applicazione di tecniche capaci di preservare l'integrità dei dati e mantenere inalterate le tracce di controllo può assicurare che l'oggetto digitale sia rimasto invariato (ad eccezione di azioni di conservazione necessarie) dal momento in cui è stato depositato in un archivio (si veda 
Fixity e checksum, e Sicurezza delle informazioni). Infine la sua autenticità per l'utente potrebbe dipendere molto di più dall'affidabilità generale dell'organizzazione che si occupa della conservazione. Sarà fondamentale il mantenimento di processi di conservazione di alta qualità basati su buone pratiche e approvati da certificazione e audit esterni specifici (si veda Audit and certificazioni).

\section{Mantenere il contesto dei dati e le loro dipendenze}

II significato delle informazioni digitali può dipendere da informazioni aggiuntive che possono essere implicite all'interno dei contesti nei quali erano state originariamente create o utilizzate, ma poco chiare quando consultate in una data successiva. L'identificazione, la comprensione e l'acquisizione di informazioni contestuali rilevanti può diventare fondamentale per un'attività di conservazione di successo. Ciò potrebbe essere semplice come l'acquisizione di unità di misura usate in un foglio di calcolo, una scala di una mappa, o il punto d'origine nei disegni CAD. Dal momento che l'informazione continua ad essere creata in modi sempre più complessi e interconnessi, potrebbe diventare necessario preservare uno spazio alle risorse digitali all'interno di un contesto di risorse informative associate più ampio. Quello che può sembrare un documento semplice potrebbe invece dipendere da relativi file, font di riferimento o potrebbe avere dei collegamenti con le rispettive informazioni sul web. Quello che potrebbe essere visto come una semplice pagina web potrebbe essere stata generata in tempo reale da dati live presi da posizioni diverse su Internet.

Capire i dati, il modo in cui vengono usati, le loro dipendenze e i contesti permetterà di acquisirli per la conservazione in modo appropriato e documentato in una modalità sufficientemente esplicita da permettere la conservazione del contenuto intellettuale e la loro comprensione nel futuro (si veda Metadati e documentazione).

\section{Agire tempestivamente}

Dare la priorità alle attività di conservazione digitale e applicarle in modo tempestivo può essere essenziale non solo al fine di evitare perdite, ma anche per assicurare il migliore utilizzo di risorse limitate. Laddove ci sia la possibilità di intervenire in via preventiva nel ciclo di vita, le risorse digitali possono essere create con l'obiettivo della conservazione nel lungo periodo. La scelta del formato dei file, l'acquisizione di documentazioni importanti o la descrizione di relazioni chiave nei metadati, possono richiedere un piccolo investimento nei costi, ma potrebbero portare a considerevoli risparmi nel lungo periodo (si veda Creazione di risorse digitali). Quando ciò non è possibile, e una volta identificati i rischi per i dati, la tempistica migliore per un'attività di conservazione può non risultare chiara. Gli interventi preliminari per affrontare l'obsolescenza tecnologica potrebbero fornire una sicurezza nella sostenibilità a lungo termine, ma con il rischio che questi interventi potrebbero, in definitiva, non essere necessari e le risorse venire sprecate. Agire per tempo potrebbe limitare attività non necessarie, ma comporta uno sforzo aggiuntivo nei casi in cui si ha a che fare con tecnologie obsolete che prevedono un tipo di conoscenza specialistica non più attuale. L'azione più appropriata dovrebbe essere intrapresa caso per caso. 


\section{Gestire la valanga di dati}

Una ricerca riportata da David Rosenthal ha evidenziato che il tasso di creazione di dati sta aumentando del $60 \%$ ogni anno, che gli sviluppi nell'archiviazione dei dati stanno aumentando di circa il $25 \%$ all'anno, e che i budget dei centri di dati stanno aumentando di circa il $2 \%$ all'anno (Rosenthal, 2014). Questi dati da una parte configurano le pressioni sulle politiche di selezione e su altri aspetti legati alle decisioni organizzative da prendere, dall'altra sollevano questioni tecnologiche. I processi di conservazione che funzionano in modo efficace su un solo livello non saranno necessariamente estesi per lavorare su ingenti volumi di dati, o eventualmente su singoli file molto grandi. La tecnologia e la constatazione che si lavori su larga scala rappresentano un processo in rapida evoluzione insieme a una maggiore competenza nel modo di gestire ampie collezioni audiovisive, dati della ricerca e archivi basati sul web (si veda Conservazione dei contenuti specifici). Ciononostante, alcuni archivi stanno ancora affrontando importanti difficoltà nello sviluppo e nel mantenimento di architetture e procedure scalabili per gestire volumi di dati in aumento. Non dovrebbero essere sottovalutate le difficoltà tecniche $\mathrm{e}$ manageriali nell'aderire, gestire e dare accesso alle risorse digitali su questa scala. Può essere importante ricordare che la selezione, la valutazione e la messa a disposizione sono dei componenti fondamentali in ogni attività di gestione del digitale. 


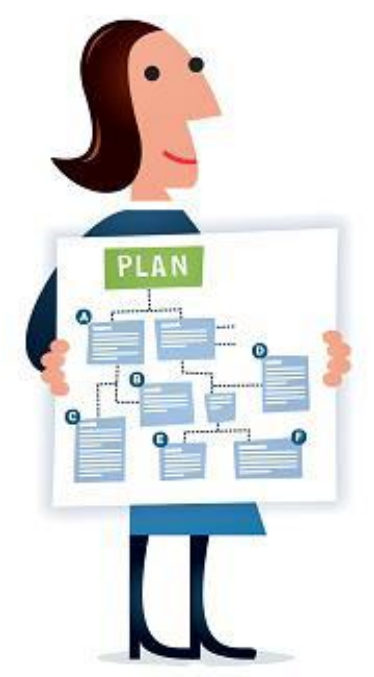

Illustrazione di Jørgen Stamp digitalbevaring.dk CC BY 2.5 Denmark

Se da una parte le problematiche tecnologiche possono risultare complicate, ci sono anche numerose criticità legate agli aspetti organizzativi. Queste includono il modo in cui la conservazione digitale viene organizzata ed eseguita, o come le responsabilità cambiano sia nel tempo che durante il ciclo di vita delle risorse digitali. Le organizzazioni affrontano problematiche comuni legate alla conservazione digitale, anche se poi ogni contesto organizzativo sarà differente. È fondamentale stabilire dei driver organizzativi e personalizzare soluzioni pratiche per andare incontro a queste necessità. Non esiste un approccio valido per tutti che integri tutti gli approcci per la conservazione digitale.

La creazione, la conservazione delle risorse digitali e il loro accesso vengono distribuiti su larga scala. Di conseguenza c'è sempre più bisogno di superare i confini delle singole organizzazioni, o persino dei Paesi, per massimizzare i benefici tecnologici, far fronte a problematiche comuni, e superare le difficoltà in un modo più redditizio.

\section{In loco o esternalizzare}

Decidere se svolgere tutto o parte dell'attività di conservazione digitale attraverso parti terze o internamente, o magari mediante una loro combinazione, è una questione complessa. La conservazione digitale potrebbe essere eseguita all'interno delle proprie organizzazioni se personale e infrastrutture fossero sufficienti, anche se esternalizzare alcune attività o supporto potrebbe avere un buon rapporto costo/efficacia e potrebbe anche essere un momento di sviluppo delle competenze interne.

Esternalizzare specifiche attività o servizi da parte di un archivio non rappresenta per nulla un fenomeno nuovo. Infatti, è da diversi anni che gli archivi esternalizzano alcune delle loro attività. Ciò che è importante è disporre comunque di competenze sufficienti per definire le specifiche e monitorare l'esecuzione dei lavori. 
L'esternalizzazione deve essere facilmente verificata così come la qualità, e ciò si può fare più facilmente attraverso una meticolosa progettazione delle specifiche e attraverso la comunicazione fornita da parti terze. I costi saranno ovviamente un punto chiave da cui partire per decidere se esternalizzare o meno il processo di conservazione digitale, ma ci sono anche altri fattori da tenere in conto come quelli legali. Ad esempio, le disposizioni di legge legate alla privacy e alla confidenzialità potrebbero incidere sul considerare l'esternalizzazione appropriata o meno. I vantaggi e gli svantaggi di ogni alternativa dovranno essere valutati in vista della missione e delle responsabilità della singola organizzazione (si veda Acquisizione e servizi di parti terze e Servizi Cloud).

\section{Collaborazione}

Esiste una sovrapposizione significativa tra le questioni legate alla conservazione digitale affrontate da tutte le organizzazioni e in tutti i settori, perciò è ragionevole mettere insieme competenze ed esperienze. Ci sono motivazioni costrittive, e in alcuni casi pressioni politiche, che fanno sì che si debba avviare una collaborazione entro e tra le organizzazioni in modo da far fronte e superare le difficoltà relative alla conservazione digitale in modo efficace.

Molte organizzazioni riconoscono prontamente i benefici di questa crescente collaborazione, ma indicano anche delle difficoltà potenziali che possono emergere sotto forma di agende differenti, tempi, o meccanismi di finanziamento. Ciononostante, è spesso possibile collaborare in specifici ambiti o su livelli diversi di intensità che moderano queste possibili difficoltà. Alcune tra le iniziative recenti con profili autorevoli e di successo nella conservazione digitale sono caratterizzate da una natura collaborativa (si veda Collaborazioni).

\section{Cambiamenti organizzativi}

L'attuale universo digitale è un luogo costituito da rapidi cambiamenti sia dal punto di vista tecnologico che organizzativo. Le organizzazioni si ristrutturano al loro interno, si uniscono, o, sempre più spesso, interrompono il loro lavoro. La conservazione digitale è un'attività di lungo periodo e la probabilità che venga condizionata da cambiamenti organizzativi aumenta nel tempo. Ciò potrebbe riguardare un archivio non solo in base a dei cambiamenti avvenuti nell'organizzazione principale, ma anche a quelli subiti dai maggiori detentori e utenti, fornitori, o collaboratori. II mutamento organizzativo rappresenta, quindi, il rischio principale da dover gestire (si veda Gestione del rischio e cambiamento).

\section{Strutture organizzative}

La natura della tecnologia e le dipendenze nella conservazione delle risorse digitali sono tali da produrre delle ripercussioni nelle strutture organizzative. Molte attività convergono, ad esempio le decisioni che riguardano l'acquisizione e la conservazione possono essere prese opportunamente allo stesso tempo. Le strutture organizzative avranno bisogno di superare i propri confini per attingere alla gamma completa di competenze e capacità richieste per le risorse digitali. L'assegnazione di responsabilità nella conservazione di risorse digitali acquisite e/o create da un'organizzazione prevedrà inevitabilmente il coinvolgimento di personale proveniente da diversi ambiti dell'organizzazione che lavorerà in forma congiunta. Ciò potrebbe 
presentare potenzialmente delle problematiche salvo che non si rafforzi una forte visione aziendale che deve essere comunicata al personale (vedi Collaborazioni, $\underline{\text { Sostegno, and }}$ Formazione del personale e sviluppo).

\section{Ruoli e responsabilità}

Esistono repository responsabili di alcune aree tematiche specifiche o formati specifici. Nel Regno Unito, ad esempio, lo UK Data Service è responsabile dei dati della ricerca delle scienze sociali, mentre il National Sound Archive della British Library lo è della sua collezione di registrazioni audio. Ogni repository dovrà considerare le proprie politiche relative alle collezioni di interesse e il più ampio panorama di istituzioni di collezioni e competenze entro le quali si trova.

II contesto digitale prevede il coinvolgimento di un ampio numero di stakeholder. L'approccio basato sul ciclo di vita della conservazione digitale consigliato in questo Manuale comporta implicazioni significative sul modo in cui le organizzazioni responsabili della conservazione a lungo termine hanno bisogno di interagire e collaborare con i creatori, gli editori e altri intermediari, nonché tra di loro.

I creatori delle risorse digitali devono essere in grado di comprendere le conseguenze delle loro azioni in termini di fattibilità a medio-lungo termine sulle risorse digitali da loro generate. Che si tratti di un record creato durante l'attività ordinaria di una azienda, di una copia digitale di materiale analogico, o di una risorsa "nativa digitale", la guida e il supporto, così come una appropriata infrastruttura tecnico-organizzativa, faciliteranno in modo decisivo il raggiungimento di prospettive per una efficiente gestione e conservazione (si veda Creazione di risorse digitali).

\section{Selezione}

L'enorme mole di informazioni che viene prodotta digitalmente, la sua qualità mutevole, e le limitazioni in termini di risorse per chi è responsabile di preservare l'accesso nel lungo periodo, rendono inevitabile un processo di selezione se l'obiettivo è quello di mantenere un'accessibilità protratta nel tempo.

Nell'ambiente digitale la scelta di non selezionare un oggetto per la conservazione potrebbe sicuramente comportarne la perdita, sebbene poi possa rivelarsi conveniente.

Nei casi in cui vi siano molteplici versioni, bisogna selezionare quale versione sia la migliore per la conservazione, o se si può scegliere anche più d'una. Fare campionamenti di risorse in evoluzione piuttosto che tentare di salvare ogni cambiamento potrebbe rappresentare l'unica alternativa pratica, ma con severe ripercussioni se la selezione non viene eseguita su un sistema chiaramente delineato e calibrato non solo sugli attuali bisogni degli utenti ma anche su quelli futuri.

Alcune considerazioni sulla selezione necessaria per assicurare la conservazione digitale: bisogna comprendere bene chi si assumerà delle responsabilità e per quale periodo di tempo, 
altrimenti, anche se vengono conservate diverse copie in diversi archivi, a un certo punto, per una serie di motivazioni, tutti quegli archivi potrebbero decidere un contemporaneo scarto dell'oggetto digitale (vedi anche Acquisizione e valutazione).

\section{Bilanciare sicurezza e accessi}

È sempre esistito un forte legame tra conservazione e accesso. Gli archivi devono far sì che le loro risorse digitali siano integre e sicure, ma soprattutto garantire l'accesso a una molteplicità di utenti. L'accesso da parte di un utente può orientare in modo significativo la progettazione delle strutture di conservazione, aiutare ad evitare delle azioni non necessarie, ma anche validare e introdurre un ciclo di feedback.

Molte tipologie di risorse digitali selezionate per la conservazione nel lungo periodo potrebbero contenere delle informazioni confidenziali e private che devono essere protette nei confronti di un accesso non autorizzato da parte degli utenti. In altri casi vi sono specifici obblighi legali o normativi sull'accessibilità degli archivi. Possono emergere delle tensioni tra questi due ruoli $e$ l'esigenza di fissare un punto d'equilibrio tra la sicurezza e la facilità d'accesso (si veda Accesso, e Sicurezza delle informazioni).

\section{Conformità giuridica}

Le problematiche legali nell'ambito della conservazione digitale non sono semplici. Si trovano spesso copie multiple e più versioni di singole risorse digitali, e insieme a queste, possono esservi software associati e metadati provenienti da fonti diverse. II contenuto digitale viene generato da un più ampio gruppo di creatori e coinvolge più formati e diritti di proprietà intellettuale (Intellectual property rights (IPR)) di quelli che si utilizzano nell'ambiente analogico. Le norme sono spesso un passo indietro rispetto ai cambiamenti della tecnologia e alle esigenze legate alla conservazione digitale. Alcuni elementi chiave che impattano sugli archivi e sulle collezioni, sulla conservazione e sulle disposizioni di accesso alle risorse digitali sono:

- Qualsiasi requisito legale in termini di gestione, conservazione e accesso relativo a un archivio e alla sua organizzazione principale, sia che provenga da donatori o finanziatori attraverso contratti e accordi sia da regolamenti da parte del Governo (es., l'accessibilità, la disponibilità, la Sicurezza delle informazioni, il mantenimento, audit e conformità, record pubblici, deposito legale, ecc.);

- Qualunque obbligo relativo ai diritti delle parti terze nelle e sulle risorse digitali detenute dall'archivio (ad es., copyright, protezione dati);

- Qualunque rapporto contrattuale tra un archivio e ogni singolo fornitore/i di parti terze (ad es., termini di servizio nei contratti e accordi sul livello dei servizi)

Per ulteriori linee guida e risorse che possono aiutare a trattare queste questioni e gestire i rischi ad esse associati, si vedano rispettivamente le sezioni del Manuale Conformità giuridica e Acquisizione e servizi di parti terze. 


\section{Finanziamenti}

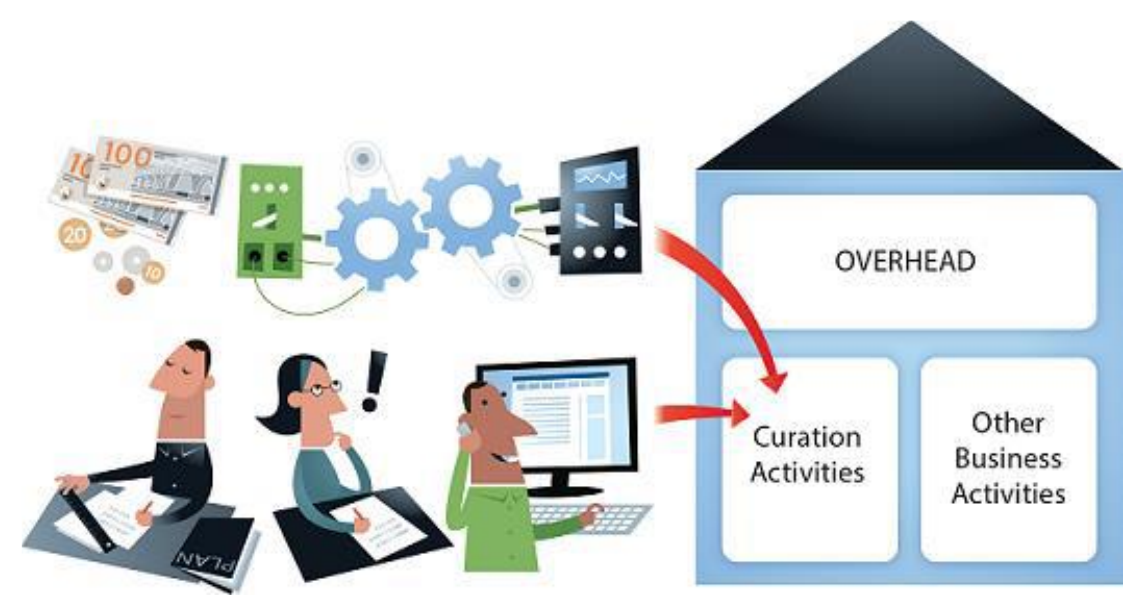

Illustrazione di Jørgen Stamp digitalbevaring.dk CC BY 2.5 Denmark

\section{Budget e costi}

Il costo della conservazione digitale non può, e non dovrebbe, essere considerato a parte così facilmente dalle altre spese organizzative. La conservazione digitale si basa essenzialmente sul mantenimento dell'accesso nel tempo e, di conseguenza, i costi per tutte le parti del ciclo di vita digitale sono importanti. In questo scenario, persino i costi legati alla creazione di risorse digitali sono fondamentali nella prospettiva in cui potrebbero dover includere delle partizioni di costo che in ultima istanza agevoleranno la loro conservazione nel lungo periodo (si veda $\underline{\text { Creazione }}$ di risorse digitali).

La capacità di disporre e formare personale con delle competenze appropriate è resa ancora più difficile dai rapidi cambiamenti della tecnologia e dall'insieme di competenze richieste. Viene anche limitata dalla mancanza di risorse delle organizzazioni che potrebbero sicuramente dover gestire collezioni tradizionali in aumento e collezioni digitali senza risorse supplementari.

Tuttavia, calcolare i costi, per quanto complesso, è un'operazione preziosa e necessaria al fine di determinare un rapporto ottimale costo/efficacia e un modello di business affidabile. II costo del lavoro richiesto per la conservazione digitale sarà di gran lunga il più significativo e include non solo esperti specializzati, ma anche l'apporto di altre competenze come quelle relative all'amministrazione, la direzione, I'ICT, i consulenti legali ecc.

Altri elementi d'impatto sui costi sono relativi alla missione aziendale e agli obiettivi, ivi compresi il tipo e la misura delle collezioni, il livello di impegno nella conservazione, la quantità e il livello di accesso necessario, e il lasso di tempo proposto per l'azione. Tutto questo viene discusso nel dettaglio nella sezione su Casi aziendali, benefici, costi e impatto. 
II rapporto tra i costi, le strategie istituzionali e attività come Collaborazioni, Acquisizione e $\underline{\text { servizi di parti terze, }} \underline{\text { Conformità giuridica, Formazione del personale e sviluppo, o Standard e }}$ buone pratiche, viene trattato nelle principali sezioni del Manuale.

\section{Personale e competenze}

La conservazione digitale coinvolge una serie di competenze e ruoli organizzativi. Generalmente, la conservazione digitale si avvale di una gamma di competenze che normalmente non si trovano in combinazione. Ciò significa che organizzazioni più grandi potrebbero aver bisogno di riunire dei gruppi interdisciplinari, mentre per quelle a dimensioni più ridotte sarà necessario affidarsi a team distribuiti o competenze esterne.

Tre sono gli aspetti da considerare in relazione al personale e alle competenze:

- Primo, nonostante i notevoli miglioramenti avvenuti negli ultimi anni, la formazione nel campo della conservazione digitale risulta spesso in ritardo rispetto alle recenti buone pratiche o è completamente teorica all'interno di programmi di gestione dell'informazione rivolti a chi si avvicina per la prima volta a questo settore. Perciò, sono altamente richieste persone con competenze pratiche ed esperienza, e il personale necessario non si riesce a trovare facilmente.

- In secondo luogo, le descrizioni delle professioni possono risultare difficili da mettere a punto specialmente quando si tratta di agenzie che stanno iniziando ex novo sulla base di nuovi ruoli. A questo scopo, diversi progetti di ricerca hanno cercato di descrivere le competenze generali richieste per la conservazione digitale usando come base l'assunto che molteplici competenze siano richieste ai diversi livelli di un'organizzazione. Strumenti come DigCurv Skills framework, legati alla sezione Digital Preservation Coalition's Vacancies, possono tornare molto utili nella descrizione dei nuovi ruoli. Le organizzazioni più grandi con gruppi interdisciplinari possono essere in grado di trovare ruoli adatti ad essere varianti "digitali" di categorie professionali esistenti, come gli archivisti, bibliotecari, o gestori di archivi, tuttavia è necessario creare nuove tipologie di ruoli per la maggior parte delle organizzazioni.

- Infine, il personale che lavora per la conservazione digitale redige con frequenza una relazione sulla necessità di investire sugli sviluppi nella carriera. Assodato che la tecnologia e i bisogni degli utenti evolvono nel tempo, anche il personale che deve ottemperare ai nuovi cambiamenti dovrà trovare delle modalità per rendere le sue competenze sempre aggiornate, attraverso, per esempio, briefing specialistici e networking professionali (si veda Formazione del personale e sviluppo).

\section{Infrastrutture}

Una conservazione digitale efficace prevede alcune infrastrutture e alcuni servizi di base che sono tipicamente tecnologici e sui quali i flussi di lavoro operativi e il processamento delle risorse digitali possono fondarsi. Sebbene queste ultime possano risultare rudimentali, o quantomeno a dimensioni ridotte nelle prime fasi in cui un'organizzazione inizia la conservazione digitale, 
I'intensificarsi di operazioni volte a trattare grandi quantità di dati richiederà un tipo di investimento considerevole nelle infrastrutture necessarie a supportare questo processo.

\section{Archiviazione}

Al fine di replicare i dati conservati per non causare perdite, l'archiviazione hardware rimane tra i più importanti servizi della conservazione digitale. La tecnologia di archiviazione è cambiata velocemente negli ultimi decenni. Gli archivi hanno usato molto supporti come CD o DVD per l'archiviazione nel lungo periodo, ma i rapidi sviluppi avvenuti tra i supporti magnetici hanno portato ad avere degli strumenti di archiviazione rapidi e affidabili che hanno reso i supporti portatili ridondanti. I sistemi di archiviazione aziendali ad oggi offrono grandi volumi di archiviazione a costi contenuti. Pur con una durata di vita limitata, generalmente intorno ai 4-8 anni, sono facilmente controllabili e sostituibili nel momento in cui raggiungono la fine del ciclo (si veda Archiviazione).

Le organizzazioni possono anche prendere in considerazione i servizi cloud per "noleggiare" le infrastrutture di conservazione. La flessibilità del cloud permette di effettuare in modo relativamente rapido le fasi di test e piloting. I servizi cloud possono offrire una replicazione facile e automatizzata su diverse postazioni, un accesso ad archivi digitali gestiti a livello professionale e un controllo di integrità. Gli archivi possono aggiungere degli strumenti dedicati, delle procedure, dei flussi di lavoro e dei contratti di servizi, garantendo un sistema digitale di archiviazione adattato alle esigenze della conservazione digitale tramite fornitori specializzati (si veda Servizi Cloud).

\section{Sistemi di archiviazione digitale}

Specifici sistemi di conservazione digitale, o archivi digitali attendibili, forniscono in modo automatico molti dei principali requisiti per la conservazione delle risorse digitali. Un'applicazione di archiviazione identificherà univocamente ogni oggetto digitale che viene inserito. Gestirà l'archiviazione di quell'oggetto, rileverà le sue caratteristiche e aiuterà il gestore dell'archivio a organizzare la sua conservazione. Inoltre, agevolerà l'accesso all'oggetto. Mentre la conservazione di base può essere effettuata in modo mirato su scala ridotta, un'applicazione specifica di archiviazione è essenziale nella gestione efficace delle risorse digitali nel tempo.

II modello OAIS offre un modello di alto livello per le funzioni richieste da un archivio (si veda Audit e certificazioni per più informazioni sulle certificazioni di archivi digitali affidabili e Strumenti per i sistemi di archiviazione e componenti).

\section{Infrastrutture informatiche ad alte prestazioni}

Per aumentare il volume dei dati c'è bisogno non solo della disponibilità di più archivi, ma anche di una capacità computazionale maggiore. L'individuazione e valutazione delle caratteristiche dei dati, l'indicizzazione volta a permettere la ricerca e gli accessi, il controllo della qualità e una serie di altre attività prevedono importanti prestazioni computazionali. Le attività che si occupano di queste grandi quantità di dati, che sia ricerca di dati o archivi web, generalmente mirano ad 
alte prestazioni informatiche, e le tecnologie come Apache Hadoop girano su gruppi di hardware di largo consumo per andare incontro a questa necessità.

\section{Laboratorio di conservazione digitale}

Diverse organizzazioni di grandi dimensioni hanno creato degli ambienti di laboratorio entro i quali possono essere applicate tecnologie consolidate o innovative per la stabilizzazione o la copia dei dati da media obsoleti, e tutton questo processo è promosso da organizzazioni che hanno a che fare con collezioni digitali personali. Supporti specifici per la lettura di supporti magnetici, robot per il processamento di numerosi dischi ottici e il blocco dei diritti di scrittura per permettere l'accesso ai dischi rigidi senza modificare i bit nel processo sono solo alcuni degli strumenti che potrebbero essere utili. Le aziende specializzate nel ripristino dei supporti possono rappresentare un approccio alternativo che potrebbe essere quello di esternalizzare il servizio - sebbene con minori controlli - in presenza di volumi rilevanti (si veda Informatica forense).

\section{Risorse}

Toy story 2 ha rischiato di essere cancellato: storie di Pixar Animation: ENTV https://www.youtube.com/watch?v=8dhp 20j0Ys

Una storia divertente e informativa su come 'Toy Story 2' venne quasi rimosso dai computer della Pixar Animation durante le riprese del film, e su come il film poi venne salvato da un computer di una delle madri dello staff ( 2 minuti e 26 secondi)

\section{Riferimenti}

Rosenthal, D., 2014. Talk "Costs: Why Do We Care?", DSHR's Blog, Martedì, 18 Novembre 2014. Disponibile al link: http://blog.dshr.org/2014/11/talk-costs-why-do-we-care.html 


\section{Guida introduttiva}

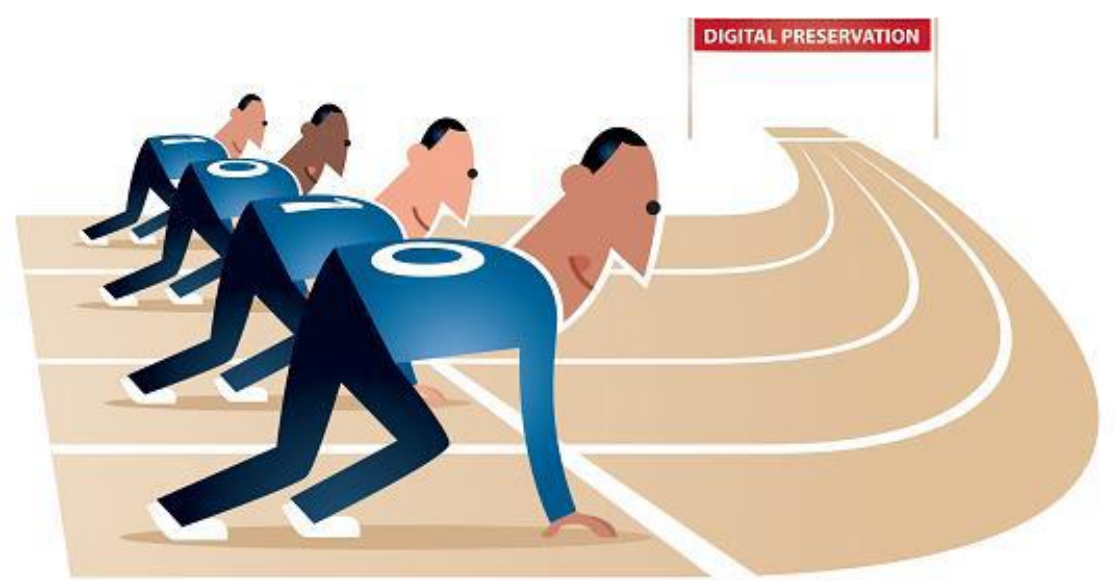

Illustrazione di Jørgen Stamp digitalbevaring.dk CC BY 2.5 Denmark

\section{Introduzione}

Questa sezione è specifica per chi deve ancora avviare un processo di conservazione digitale o per chi ha appena iniziato a farlo, presenta una rapida introduzione a una serie di approcci che aiuteranno ad avviare gli utenti, ad introdurli alle altre sezioni del Manuale e a diventare più indipendenti e acquisire le competenze necessarie.

La sezione è stata creata a partire dai workshop "Guida introduttiva alla conservazione digitale" organizzati dalla Digital Preservation Coalition. Supporta l'approccio "imparare facendo" e prevede un livello minimo di conoscenza. Man mano che si andrà avanti si noteranno i vantaggi apportati dalle risorse e dai casi di studio, da altri argomenti e sezioni del Manuale, e dal Glossario, da utilizzarsi per qualsiasi termine che non risulti familiare.

La conservazione digitale può apparire come una prospettiva scoraggiante. Può aiutare a mappare le proprie competenze e risorse con i materiali che si vogliono conservare. In tal modo si può iniziare con quello che già si sa e non con ciò che non si conosce. Il primo passaggio nella conservazione digitale quasi sempre prevede una rapida valutazione. Questa fase è costituita da due o tre elementi:

- conoscere la capacità operativa della propria organizzazione;

- individuare gli obiettivi e le finalità dell'organizzazione;

- conoscere le risorse digitali da conservare.

\section{Conoscere la propria organizzazione e i propri dati}

\section{Creare un Digital Asset Register}

Parte di una rapida valutazione comprende la definizione della natura e dell'ampiezza delle proprie collezioni digitali. Un Digital Asset Register sarà veramente utile nella valutazione dell'ampiezza e della significatività della collezione, nell'identificazione delle priorità e 
pianificazione di azioni di conservazioni digitali. In seguito verrà descritto meglio il processo di valutazione ad alto livello della collezione: un controllo approfondito e dettagliato potrebbe portare via molto tempo. Dunque, il consiglio in queste prime fasi è quello di mantenere l'asset register semplice e porsi le seguenti domande:

- Qual è l'argomento della collezione?

- Da dove proviene e qual è la sua funzione?

- Dov'è archiviata e quali tipi di supporti sono stati usati?

- Perché è in conservazione?

- Chi ne è il responsabile; chi sono gli utenti; a chi sono rivolti i dati?

- Come avviene l'accesso ai dati?

- Come potrebbero cambiare e crescere i dati in un futuro prossimo?

Valutare la preparazione dell'organizzazione

La maturità organizzativa è un altro fattore da tenere in conto. In America, la National Digital Stewardship Alliance (NDSA, 2013) ha indicato un modello a 4 livelli per aiutare le organizzazioni a comprendere e migliorare le proprie competenze tecniche nella conservazione digitale:

- Livello 1 - proteggere i propri dati

- Livello 2 - conoscere i propri dati

- Livello 3 - monitorare i propri dati

- Livello 4 - riparare i propri dati

Questi 'Livelli di Conservazione' si devono considerare in prospettiva, e sono usati per misurare il livello di maturità su cinque elementi: archiviazione, fixity dei file, sicurezza delle informazioni, metadati e formati dei file. La capacità organizzativa per avviare un processo di conservazione digitale viene indicata a partire dal suo livello di maturità su questi cinque elementi. Sono disponibili modelli più esaustivi, come ad esempio il Digital Preservation Capability Maturity Model (Dollar and Ashley, 2014), qualora si necessitasse di un tipo di esplorazione più approfondita della maturità organizzativa.

\section{Primi passi verso la messa in sicurezza dei propri dati}

In questa sezione si descrivono le azioni preliminari da compiere per mettere al sicuro i propri dati una volta attestata la preparazione delle organizzazioni e fornito le informazioni di base. I passaggi seguenti sono essenziali per garantire un minimo livello di conservazione nel momento in cui sopraggiunge una nuova collezione di risorse informatiche. Generalmente, ciò si riferisce alla conservazione dei bit, e letteralmente significa conservare i flussi di cifre binarie, o bit, che formano i propri file digitali (senza conservare gli strumenti per la decodifica dei bit in informazione significativa). 


\section{Immediata verifica della ricezione}

Quando una nuova collezione di risorse digitali arriva da parte di un fornitore, è essenziale assicurarsi che ciò che è stato ricevuto equivalga a ciò che si stava aspettando. A seconda della fonte della risorsa è possibile richiedere nuove copie di file mancanti o di scarsa qualità. Questi controlli vengono effettuati così come qualsiasi richiesta di sostituzione proposta, in modo da garantire una risoluzione efficace.

Le principali attività prevedono:

- Scansione di virus o malware per assicurare che non vi siano sorprese inaspettate nella collezione. Si può tenere eventualmente la collezione 'in quarantena/isolamento' fino a quando la si verificherà.

- Controllo di tutti i file previsti presenti. Se la risorsa è seguita da un indice, si dovrebbe verificare il file in relazione a quest'ultimo.

- Apertura di una selezione casuale dei file per verificarne l'integrità e/o i livelli di qualità previsti.

- Ove possibile, richiesta repentina di sostituzioni per qualsiasi danno o file mancanti.

\section{Creazione di una Lista file verificabile}

Per controllare nel corso del tempo che i propri file digitali siano stati conservati, è anzitutto necessario registrare in maniera esatta quali sono i file in proprio possesso. Perciò, è importante creare una lista attendibile di file presenti in ogni collezione. Queste liste dovrebbero presumibilmente contenere informazioni quali i nomi dei file, la posizione e le dimensioni, le tipologie di formati e il checksum. Un checksum è una stringa alfanumerica breve che rappresenta i contenuti dei file ed è una sorta di 'impronta digitale' che permette il confronto nel tempo. Una volta creata la lista, verificare se tutti i file sono presenti e non danneggiati in qualunque momento in futuro (si veda la verifica della fixity in seguito), è una attività semplice. Possono essere usati diversi software per generare automaticamente questi dati; di solito sono chiamati strumenti di caratterizzazione. Ad esempio, il National Archives PRONOM (un registro di formati dei file e i loro comportamenti) e DROID (uno strumento che usa PRONOM per analizzare il file sul sistema). Avere all'interno della propria collezione una lista di formati dei file, versioni e quantità, aiuterà a creare un caso da presentare ai decisori e le risorse che saranno richieste per svolgere il lavoro adeguatamente e in modo sostenibile. Queste informazioni possono essere anche usate per aggiornare e arricchire i propri digital asset register. La gamma dei formati che si utilizzano dovrebbe essere consolidata al fine di ridurre al minimo la duplicazione ed eliminare i problemi legati ai formati. Questo processo è noto come normalizzazione.

Tra le attività principali vi sono:

- Generazione di una lista di file attendibile

- Aggiornamento del digital asset register. 


\section{Stabilizzare i propri file: fare delle copie}

A prescindere da quanto sia valido il proprio archivio digitale, le proprie risorse digitali saranno sempre a rischio di essere danneggiate, smembrate $\mathrm{o}$ accidentalmente eliminate. Fare più copie delle proprie risorse digitali e utilizzare più di una tipologia di soluzioni di archivio riduce la molteplicità dei rischi legati alla conservazione digitale.

Le principali attività prevedono:

- La conservazione di (almeno) una copia facilmente accessibile su un disco non rimovibile. Si avrà bisogno di rivedere i propri materiali per assicurarne la fixity, perciò mantenerli accessibili faciliterà questo processo.

- La creazione di (almeno) una copia in più, se necessario su un archivio meno accessibile, ma su un media con un supporto più conveniente, come ad esempio un nastro.

- Una copia posizionata in una località geografica diversa rispetto alle altre per ovviare a disastri naturali o dolosi.

\section{Riesaminare e ispezionare: verifica della fixity}

Riesaminare le proprie risorse digitali con regolarità (ad es., ogni sei mesi) può garantire che non ci siano stati danni o perdite accidentali. Se ciò è avvenuto, è possibile recuperare i file problematici dalle copie o dai backup che si sono precedentemente eseguiti. I controlli di integrità futuri genereranno nuove impronte digitali (o checksum) per i file conservati. Se questi non combaciano con quelli originariamente creati, vuol dire che si è verificata una alterazione o un danno.

Le principali attività sono le seguenti:

- Riesaminare la propria collezione di frequente, ricalcolare i checksum, identificare i file che risultano danneggiati.

- Recuperare le copie dei file danneggiati e ripararli, se necessario.

- Effettuare dei test di recupero per i dati di cui si è eseguito il backup a partire da servizi di parti terze al fine di assicurarsi che i backup siano stati eseguiti nel modo concordato.

\section{Documentare i propri processi}

Fin dall'inizio del processo di creazione di una collezione digitale è importante documentare quanto più possibile i contenuti della collezione, gli strumenti e i flussi di lavoro. Questa documentazione è un elemento importante per Metadati tecnici e descrittivi. Si deve mantenere questa informazione ai fini della longevità, dal momento che, con qualsiasi progetto, il mantenimento del personale può diventare un problema. Se il personale va via, spesso lo fa portando con sé competenze essenziali e le proprie conoscenze. 


\section{Prospettive future}

Dopo aver fatto i primi passi nella conservazione digitale, qual è il prossimo step?

Questo ovviamente dipenderà delle necessità e priorità di ciascuno, ma la seguente tabella riporta una serie di suggerimenti e altre sezioni del Manuale che aiuteranno a orientarsi da qui in avanti:

\section{Prossimi step}

Sviluppare una forma di sostegno e sensibilizzazione, una comprensione del rischio, casi aziendali, costi, benefici e impatto.

Determinare una strategia di conservazione organizzativa e politiche adeguate. Assicurare poi un approccio organico alla conservazione può essere un metodo utile da perseguire presso una qualunque organizzazione.

Definire il proprio archivio digitale. Soluzioni tecniche e strumenti sia su infrastrutture locali IT o offerte come servizi cloud potranno aiutare a capire, gestire e conservare le proprie risorse digitali a lungo termine.

Definire un archivio in una prospettiva a lungo termine e un piano di conservazione e azione.

Riesaminare e potenziare i propri audit sulla collezione:

- Caratterizzare le collezioni con priorità con più dettagli

- Aggiornare periodicamente gli audit sulle collezioni come richiesto

Costituire un gruppo di lavoro per la conservazione digitale. Una conservazione digitale efficace spesso prevede la collaborazione di diverse strutture all'interno dell'organizzazione. Un gruppo di lavoro rappresentativo può essere vitale a rendere coordinati i passaggi successivi.

Gettare le basi per una necessaria formazione e crescita del personale e delle competenze.

Definire un network professionale e di collaborazioni. Aderire a organizzazioni di conservazione come la Digital Preservation Coalition.

Mettersi al passo con nuovi aggiornamenti:

- liste di e-mail per la conservazione digitale includono, ad esempio, la lista degli annunci di conservazione digitale su JiscMail, e la lista, a impostazione statunitense, digipres;

- un blog settimanale seleziona i tweet recenti e i link delle notizie sulla conservazione digitale;

- riviste con focus sulla conservazione digitale come ad esempio: International Journal of Digital Curation, e D-Lib;

- eventi con focus sulla conservazione digitale come iPRES e PASIG;

- la Digital Preservation Coalition organizza giornate di orientamento su particolari argomenti legati alla conservazione digitale. 


\section{Risorse}

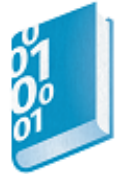

\section{A Preservation Primer}

http://knconsultants.org/a-preservation-primer/

Questo manuale introduttivo alla conservazione per utenti principianti è stato redatto dallo staff di Portico. Riporta le questioni e gli schemi sulla conservazione a breve e lungo termine che un'organizzazione potrebbe prendere in considerazione per avviare una pianificazione di conservazione a lungo termine delle sue risorse, iniziando con la protezione nel breve periodo e concludendo con la conservazione completa e a lungo termine. (83 pagine)

\section{Putting Parsimonious Preservation into Practice}

http://www.nationalarchives.gov.uk/documents/information-management/parsimonious-

\section{preservation-in-practice.pdf}

II Parsimonious Preservation è stato originariamente sviluppato nel 2009 presso il National Archives nel Regno Unito come approccio rivolto a istituti di piccole o medie dimensioni per permettere loro di iniziare ad occuparsi di conservazione digitale, ma è anche utile per istituti di grandi dimensioni. Fornisce dei suggerimenti e delle linee guida per i settori archivistici inglesi (UK) sulla conservazione digitale. (11 pagine).



\section{Community Owned digital Preservation Tools Registry COPTR}

\section{http://coptr.digipres.org/Main Page}

COPTR presenta gli strumenti utili per la conservazione digitale a lungo termine e rappresenta uno strumento di rilevamento e valutazione per aiutare i professionisti del settore a trovare gli strumenti di cui hanno bisogno per conservare i dati digitali. II suo scopo è di collazionare in un unico luogo la base di conoscenza della comunità di conservazione digitale sugli strumenti di conservazione. Inizialmente includeva dati dai registri gestiti dalle organizzazioni partner COPTR, tra cui quelle gestite da Digital Curation Centre, Digital Curation Exchange, National Digital Stewardship Alliance, Open Preservation Foundation, e Preserving digital Objects With Restricted Resources project (POWRR). COPTR rileva dettagli di base e concreti su uno strumento, evidenzia ciò che uno strumento fa e scopre come ottenere più informazioni (URL rilevanti) e riferimenti per le esperienze che gli utenti hanno con i tool. L'ambito fa riferimento a un'interpretazione generale del termine 'conservazione digitale', in altre parole, se un tool è ritenuto efficace nelle prestazioni di conservazione digitale come quelle descritte nel modello OAIS o nel modello del ciclo di vita DCC, allora rientra nell'ambito di questo registry. 


\section{DPC Getting Started in Digital Preservation Workshops}

https://www.dpconline.org/knowledge-base/training/training-resources

I workshop DPC Getting Started in Digital Preservation sono eventi che mirano a sensibilizzare il pubblico sugli aspetti legati alla conservazione digitale, sul miglioramento del livello di coinvolgimento nelle attività di conservazione digitale, sulle forme di sostegno e risorse disponibili a facilitare questo processo. Forniscono un'introduzione alla conservazione digitale, creano consapevolezza sui rischi legati alle risorse digitali, comprendono sessioni pratiche per aiutare gli utenti ad applicare i piani e gli strumenti di conservazione digitale, e coinvolgono speaker per condividere delle esperienze personali relative all'implementazione della conservazione digitale. Dettagli sui prossimi workshop, programmi, presentazioni di speaker nei workshop precedenti, si possono trovare sulla pagina degli eventi DPC.

Digital Preservation Management: Implementing Short-Term Strategies for Long-Term Solutions

dpworkshop.org/

Un ottimo tutorial ad accesso libero online che introduce ai principi cardine della conservazione digitale. Rivolto principalmente ai bibliotecari, archivisti, curatori, direttori, e professionisti tecnici, include definizioni, concetti chiave, suggerimenti pratici, esercizi, riferimenti aggiornati. II tutorial è disponibile in inglese, francese e italiano.

\section{Canadian Heritage Information Network (CHIN) Digital Preservation Toolkit} https://www.canada.ca/en/heritage-information-network/services/digital-

\section{preservation/toolkit.html}

CHIN ha pubblicato una suite di documenti per l'identificazione di risorse digitali ritrovate nei musei, del rischio potenziale e dell'impatto delle risorse perdute, e un'introduzione sullo sviluppo delle Politiche di Conservazione, Piani e Procedure. II toolkit comprende il Digital Preservation Inventory Template, Digital Preservation Policy Framework Development Guideline, Decision Trees, e Digital Preservation Plan Framework.

\section{Digital Preservation 101, or, How to Keep Bits for Centuries}

https://tpverso.files.wordpress.com/2016/03/swierczek.pptx

Questa presentazione di Julie Swierczek, già Digital Asset Manager e Digital Archivist alla Harvard University Art Museums, rappresenta un supporto e una spiegazione valida della conservazione digitale a utenti non-esperti che fanno parte di organizzazioni, includendo il 'perchè gli archivisti si disperano quando poi il pubblico in generale confonde gli archivi con le copie di backup?' (81 slide, ma accompagnate da immagini con diverse note che rendono il tutto facilmente comprensibile). 


\section{The National Archives Digital Continuity Guidance}

http://www.nationalarchives.gov.uk/information-management/manage-information/policy-

\section{process/digital-continuity/step-by-step-guidance/step-2/}

Questa guida presenta il processo di creazione di uno IAR $^{20}$ (Information Asset Register), e include un template nel formato di un foglio di calcolo Excel. II registro può essere utile per il Responsabile della gestione documentale e può altresì rappresentare un modello che può, effettivamente, essere associato alla gestione del rischio aziendale.

\section{Risk Management for Digital Preservation}

https://vimeo.com/171082277

Tratt da un insieme di video che riguardano argomenti riferiti al roadshow della 'Getting Started in Digital Preservation', questo offre una breve presentazione sull'attuazione di un piano di gestione nella conservazione digitale.

\section{Casi di studio}

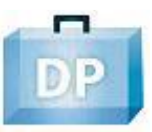

\section{Biblioteca Bishopgate}

http://wiki.dpconline.org/index.php?title=Bishopsgate library case study

Una collezione di casi di audit e casi aziendali incentrati sui primi passi nell'ambito della conservazione digitale presso la Bishopsgate Institute Library. (28 pagine).

\section{Starting Small: Practical First Steps in Digital Preservation}

http://www.slideshare.net/hakbailey/starting-small-practical-first-steps-in-digital-preservation-

\section{4}

Un esempio di come i principi legati alla conservazione digitale possano venire integrati alle attività di gestione delle collezioni appartenenti a istituti di piccole dimensioni (Dartmouth College USA dal 2010 al 2012), senza che vi sia bisogno di ulteriori risorse. (26 slide).

20 Gli IAR sono registri appositamente costituiti per acquisire e organizzare i metadati su grandi quantità di informazioni detenute da amministrazioni ed enti pubblici. Uno IAR completo include i database, i vecchi insiemi di file, i recenti file elettronici, le raccolte di dati statistici, di ricerca e così via. 
DPC case note: West Yorkshire Archive Service accepts a digital collection

http://www.dpconline.org/component/docman/doc download/511-casenotemlawyas.pdf

In un caso di studio supportato da JISC, i membri dello staff del West Yorkshire Archive Service raccontano la loro esperienza all'interno del loro primo grande archivio digitale; tale esperienza li ha messi di fronte a una serie di nuove problematiche e modi di lavorare. Concludono dicendo 'Se proviamo, potremmo fallire; Se non lo facciamo, falliremo di certo'. Ottobre 2010 (4 pagine).

DPC case note: Glasgow Museum takes first steps in turning an oral history headache into an opportunity

http://www.dpconline.org/component/docman/doc download/502-

\section{casenoteglasgowmuseums.pdf}

Questo caso di studio supportato da JISC descrive i passaggi intrapresi dal Museo di Glasgow nell'implementazione della conservazione digitale, e di come questo abbia creato opportunità a breve e lungo termine. Attività come la realizzazione di un inventario, l'attribuzione di significato e l'offerta di accesso, rappresentano la base per diventare più sicuri nell'affrontare sfide più generali, e gratificheranno se appropriatamente incorporate all'interno dell'ambito della missione di un'organizzazione. Settembre 2010 (4 pagine).

\section{Digital Preservation Planning Case Study}

http://www.dpconline.org/component/docman/doc download/863-2013-may-getting-started-

london-planning-case-study-ed-fay

Un insieme di slide di presentazione del workshop della DPC 'Getting Started in Digital Preservation' di Ed Fay nel Maggio del 2013. Un'ottima breve panoramica sulla pianificazione della conservazione digitale e su quali approcci utilizzare. (20 slide).

\section{Riferimenti}

Dollar, C.M. and Ashley, L.J., 2014. Assessing Digital Preservation Capability Using a Maturity Model Process Improvement Approach. Disponibile al seguente link: http://static1.squarespace.com/static/52ebbb45e4b06f07f8bb62bd/t/53559340e4b058b6b2212 d98/1398117184845/DPCMM+White+Paper_Revised+April+2014.pdf

NDSA, 2013. The NDSA Levels of Digital Preservation: An Explanation and Uses, version 1 2013. National Digital Stewardship Alliance. Disponibile al seguente link: http://www.digitalpreservation.gov/ndsa/working_groups/documents/NDSA_Levels_Archiving_ 2013.pdf 


\section{Strategie istituzionali}

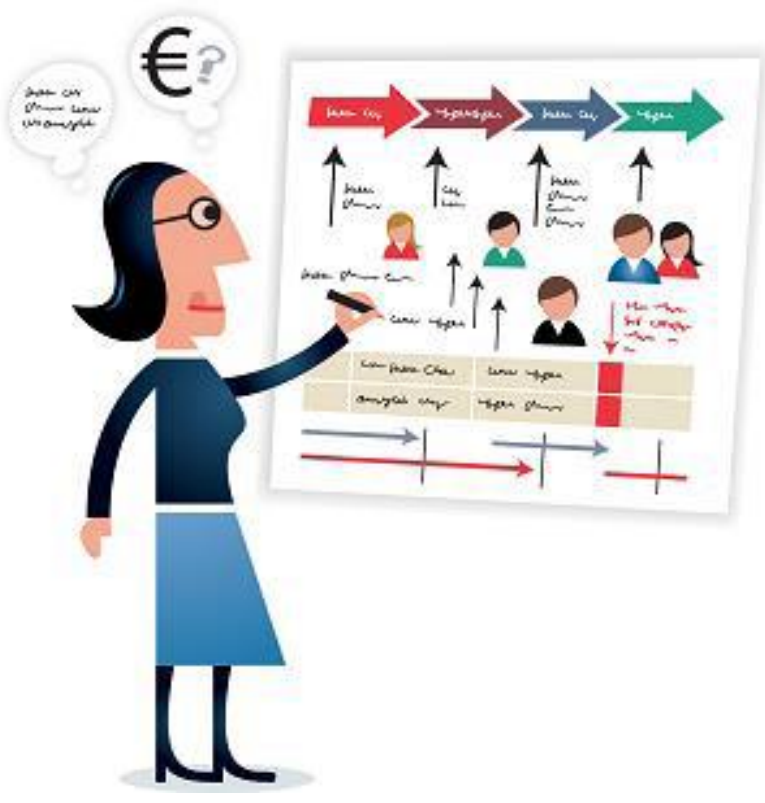

Immagine: digitalbevaring.dk CC BY 2.5 Denmark

\section{A chi è rivolto?}

Sia agli amministrativi senior (DigCurV Executive Lens) che ai direttori operativi (DigCurV Manager Lens) all'interno delle istituzioni, e anche a fornitori di servizi terzi esistenti o potenziali.

Requisiti minimi di conoscenza

Intermedio (conoscenza di base, qualche esperienza pratica).

\section{Obiettivo}

- Creare le basi per sviluppi futuri di politiche e strategie adeguate alle singole istituzioni.

- Garantire degli esempi reali di buone pratiche che potrebbero servire da modelli.

- In questa sezione si presentano delle strategie che sono state utilizzate con buoni risultati dalle istituzioni nella prospettiva di sviluppo di approcci legati alla conservazione digitale. Ogni sotto sezione descrive l'approccio, i suoi potenziali vantaggi e svantaggi, e poi se ne danno degli esempi aggiungendo letture integrative sull'argomento. Strategie come queste costituiranno un elemento centrale delle politiche di sviluppo aziendale nel campo della conservazione digitale. Una solida politica di sviluppo in combinazione con pratiche lavorative e procedure efficaci (si veda Attività organizzative) sono requisiti essenziali per un adeguato programma di conservazione digitale. 


\section{Politiche istituzionali e strategie}

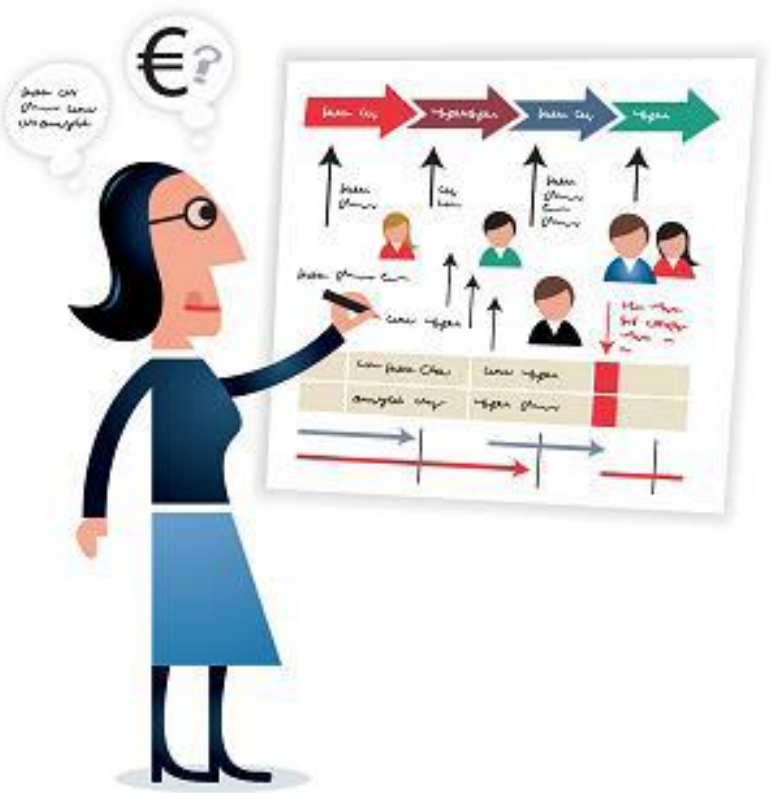

Illustrazione di Jørgen Stamp digitalbevaring.dk CC BY 2.5 Denmark

\section{Introduzione}

Lo scopo di questa sezione è quello di aiutare le istituzioni a capire, sviluppare e implementare delle politiche e delle strategie di conservazione digitale. Queste ultime aiuteranno un'organizzazione a impostare degli obiettivi di conservazione digitale, delle priorità e dei meccanismi che supporteranno, in aggiunta, la gestione del ciclo di vita e la disseminazione delle risorse digitali.

Politiche e strategie sono termini che spesso si usano intercambiabilmente o in una differente sequenza gerarchica in diverse situazioni. Per coerenza, il Manuale definisce con 'politiche' i documenti di più alto livello e con 'strategie' i documenti e le procedure che supportano l'implementazione delle politiche. In linea di principio lo sviluppo delle politiche precede lo sviluppo delle strategie. D'altra parte, le strategie potrebbero essere sviluppate o riesaminate con frequenza regolare, mentre le politiche potrebbero prevedere un ciclo di revisioni più lungo. Di conseguenza le politiche supportano le necessità organizzative, mentre le strategie individuali diverse unità o divisioni aziendali.

I documenti strategici di programmazione forniscono una base su cui tutte le attività che si occupano di gestione delle risorse digitali si fondano. Quando sono ben fatti e consultabili, garantiscono alti livelli sia di consensi che di conformità nelle attività quotidiane per la gestione di risorse digitali. A sua volta, ciò garantisce anche che le risorse digitali siano gestite in modo appropriato e con i migliori risultati. I documenti strategici di programmazione costituiscono anche la base per la pianificazione dei costi e per le richieste di finanziamento. Le strategie 
possono essere utilizzate come uno strumento flessibile sia per adattare le situazioni al cambiamento che per dar prova che ciò che è stato appreso è stato applicato.

All'interno di ogni istituzione ci sarà un numero di stakeholder con una quota di partecipazione nella gestione del ciclo di vita delle risorse digitali. Questi ultimi possono contribuire alla gestione di queste risorse, creare o gestire i metadati associati ad esse, o avere delle responsabilità di gestione per le collezioni. Anche gli utenti finali rappresentano degli stakeholder importanti poiché le loro richieste determinano cosa è importante per la conservazione. Le visioni degli stakeholder e i loro ruoli rispetto alla gestione delle risorse digitali devono essere considerate sia a livello di politica che di strategie.

Potrebbe essere utile applicare il metodo di gestione iterativa a quattro fasi del Pianifica-FaiControlla-Realizza come modello per un costante miglioramento, uno sviluppo efficace e un'implementazione e revisione della politica e delle strategie.

\section{Politiche di conservazione digitale come parte di un contesto organizzativo più ampio}

Se si sta progettando, o pensando di progettare, la messa a punto di politiche di conservazione digitale per la propria organizzazione è necessario iniziare partendo dall'indagine sul contesto in cui ci si trova ad operare.

È probabile che un'ampia gamma di documenti strategici esistano nella propria organizzazione e coprano una grande varietà di questioni legate ad esempio al personale, alla tecnologia informatica, alla valutazione del rischio, e all'aspetto finanziario. Potrebbero anche esserci delle politiche relative a situazioni più specifiche come le gestioni di record e collezioni che potrebbero essere rilevanti per le attività di conservazione digitale. Diventa fondamentale, perciò, prendere in considerazione sia il contenuto che la forma stabilita, nonché la struttura di tutte le politiche rilevanti all'interno dell'organizzazione così come il modo in cui la politica di conservazione digitale si collocherà nel panorama politico più ampio. Nessun singolo documento strategico può avere vita autonoma, perciò, per riuscire ad avere il supporto e l'implementazione di una politica di conservazione digitale è fondamentale integrarla in un contesto di politica più ampio.

\section{Importanti considerazioni nello sviluppo di politiche e strategie}

Un importante aspetto inerente alle politiche di sviluppo è la valutazione dei bisogni specifici della propria organizzazione e dei suoi principali attori. L'allineamento con gli operatori assicura che anche le strategie e la loro implementazione siano in linea con le esigenze aziendali. I documenti strategici di programmazione dovrebbero rendere chiaro il collegamento con e tra politiche e strategie rilevanti ed esistenti, nonché basarsi su prassi vigenti. La collaborazione, la condivisione e la consultazione con gli stakeholder sono dei processi fondamentali nello sviluppo di politica e strategie.

Le politiche di conservazione digitale sono idealmente neutrali dal punto di vista tecnologico, vale a dire che non dipendono da nessuna piattaforma tecnologica o sistema. Tuttavia, in realtà, questo potrebbe non essere del tutto vero, e le politiche di conservazione digitale dovrebbero essere concentrate sui principi, scopi e obiettivi che la tecnologia da utilizzare può supportare. 
Al fine di elaborare una documentazione e definire dei processi chiari, coerenti e solidi, è importante conformarsi a una metodologia impostata e stabilire un piano per la sua revisione cosicché politica e strategie possano rimanere rilevanti e attuali.

1. Stabilire un obiettivo. II primo passaggio è stabilire il principale obiettivo della politica di conservazione digitale, il suo ambito e le principali finalità. Ciò permetterà di focalizzare i contenuti delle politiche da adottare. In questa fase bisognerebbe prestare attenzione a come verrà utilizzata la documentazione, sia come strumento per la promozione che per guidare la creazione e implementazione delle strategie.

2. Ricerca. Come precedentemente menzionato, è essenziale capire il contesto organizzativo in cui le politiche avranno valore. Dovrà essere dedicato tempo all'analisi di politiche esistenti, alla comprensione di business drivers dell'organizzazione e dei bisogni dei principali gruppi di stakeholder. Questa fase comprenderà anche l'indagine delle migliori prassi per le politiche e strategie di conservazione digitale. Sono disponibili diverse risorse con dei suggerimenti su cosa includere nelle proprie scelte politiche e strategiche sulla conservazione digitale (si veda Risorse).

3. Identificare gli elementi e sviluppare la struttura. In base alla ricerca portata avanti nella fase precedente, si dovrebbero selezionare poi gli argomenti e le problematiche principali delle politiche e delle strategie. Creare una struttura chiara per i documenti è essenziale per far sì che questi risultino utili nella pratica e per facilitare semplici aggiornamenti e revisioni. La struttura dovrebbe riflettere gli standard e le migliori prassi esistenti.

4. Creare il contenuto. II contenuto politico dovrebbe essere di alto livello e definire delle finalità e degli obiettivi generali. Dovrebbe poi evitare di riconoscere le specifiche come dettagli legati a soluzioni tecnologiche proprietarie, sebbene sia possibile includere dei riferimenti a quanto necessario a livello organizzativo. Le informazioni sull'applicazione pratica delle politiche verranno definite da documenti di strategia rilevanti. II contenuto potrebbe anche essere anche una dichiarazione di intenti, ma bisogna prestare attenzione a non fissare degli obiettivi irraggiungibili. Sono disponibili varie risorse (si veda Risorse) e dei suggerimenti su come affrontare situazioni specifiche nell'ambito delle proprie politiche e strategie.

5. La revisione degli stakeholder. È essenziale ottenere il consenso di vari gruppi di stakeholder per far sì che sia le politiche che le strategie siano adatte all'obiettivo e ricevano in futuro il supporto da parte di tutta l'organizzazione. La presentazione dei progetti di documenti ai principali gruppi di stakeholder rappresenta una parte importante del processo di progettazione, e ogni riscontro che ne viene dato dovrebbe essere attentamente preso in considerazione. Questa è anche un'importante fase nella promozione della conservazione digitale all'interno della propria organizzazione, e può permettere agli stakeholder di sentirsi coinvolti nel processo e di comprendere quanto le attività di conservazione digitale siano correlate al loro lavoro (si veda Sostegno).

6. Ottenere l'approvazione. La maggior parte delle organizzazioni stabiliranno che i nuovi documenti strategici di programmazione siano ratificati ufficialmente dal proprio consiglio di amministrazione. Bisogna assicurarsi di essere al corrente del processo dell'organizzazione e di ogni necessità che dovrà essere soddisfatta. Una volta ratificate, le scelte avranno più valore e, conseguentemente, sarà più facile implementarle come parte di una strategia in corso. 
7. Revisioni periodiche. I documenti strategici di programmazione non dovrebbero essere statici, ma devono essere sensibili ai cambiamenti che avvengono nei bisogni degli stakeholder, nel contesto organizzativo più ampio e negli aggiornamenti legati alle buone pratiche. Dovrebbero essere previsti cicli di revisione periodica, ma che potrebbero essere anche attivati da significativi cambiamenti in una qualsiasi delle aree precedentemente menzionate.

8. Implementazione. Stabilire un piano di implementazione per concretizzare politica e strategie nelle operazioni giornaliere. Ma si tenga a mente che esse sono un mezzo per raggiungere un fine, e non un fine in sé.

\section{Risorse}

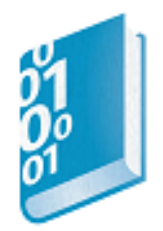

\section{Digital Preservation Policies Study}

http://www.webarchive.org.uk/wayback/archive/20140615022334/http://www.jisc.ac.uk/media/ documents/programmes/preservation/iiscpolicy p1finalreport.pdf

Questo studio finanziato da JISC e pubblicato nel 2008 ha rappresentato un modello di riferimento per le politiche di conservazione digitale e ha accompagnato le clausole d'adempimento basate sull'analisi di politiche di conservazione digitale esistenti. Nonostante sia incentrato più su settori di istruzione superiore e di formazione continua britannici, questo studio tratta in modo generale le politiche e gli adempimenti in tutti gli altri settori e Paesi.

Tra i risultati ottenuti vi è un insieme di mappature associative relative alla conservazione digitale con le strategie istituzionali principali nelle università del Regno Unito e scuole superiori con lo scopo di supportare gli istituti e il loro staff a sviluppare politiche e clausole di conservazione digitale per impostarle in un contesto di strategie istituzionali più ampio. (60 pagine).

\section{Digital Preservation Policies: Guidance for archives}

http://www.nationalarchives.gov.uk/documents/information-management/digital-preservationpolicies-guidance-draft-v4.2.pdf

Pubblicata dal National Archives nel 2011, questa guida spiega le caratteristiche principali di una strategia di conservazione digitale. Spiega il perché ci sia bisogno di una strategia politica e di come questa supporti la conservazione digitale. II pubblico di riferimento della guida sono gli archivi a finanziamento pubblico. (16 pagine).

Analysis of Current Digital Preservation Policies: Archives, Libraries and Museums http://www.digitalpreservation.gov/documents/Analysis\%20of\%20Current\%20Digital\%20Prese rvation\%20Policies.pdf?loclr=blogsig

Questo report, pubblicato nel 2013 da Madeline Sheldon, un Junior Fellow di NDIIPP alla Library of Congress, dibatte sullo stato attuale delle strategie politiche della conservazione digitale all'interno di istituti di patrimonio culturale. L'insieme delle politiche di conservazione digitale, 
nuove o recentemente aggiornate, pubblicate durante il 2008 e 2013, ha condotto a un livello alto di analisi dei contenuti all'interno di quei documenti. Una panoramica di sentesi riferita ai risultati è stata resa disponibile su un post del blog The Signal. (23 pagine).

\section{APARSEN D35.1 Exemplar good governance structures and data policies \\ http://www.alliancepermanentaccess.org/wp- content/uploads/sites/7/downloads/2014/06/APARSEN-REP-D35 1-01-1 0 incURN.pdf}

Questo report fa il punto sul livello di preparazione per una gestione interoperabile e per le politiche in materia di dati. Si conclude con una serie di raccomandazioni selezionate che dovrebbero essere seguite nel momento in cui si definiscono delle politiche in materia di dati sulla conservazione digitale. (2014, 43 pagine).

\section{SCAPE Catalogue of Preservation Policy Elements}

http://wiki.opf-labs.org/display/SP/Catalogue+of+Preservation+Policy+Elements

AI Progetto europeo SCAPE (2011 - 2014) fu affidato il compito di cercare le politiche e produrre un catalogo degli elementi riferiti alle politiche per fornire supporto a chi era incaricato di redigere queste ultime. Questo wiki fornisce alcune informazioni sul background del lavoro di policy e presenta delle pagine per ognuno degli elementi che il progetto SCAPE ha indicato alle organizzazioni di tenere in conto nel momento in cui dovevano redigere le politiche, con un focus particolare sulla pianificazione e monitoraggio delle attività. Vi è anche un report finale relativo a questo lavoro reso pubblicamente disponibile a Febbraio del 2014 sul sito web di SCAPE.

\section{Published Preservation Policies \\ http://wiki.opf-labs.org/display/SP/Published+Preservation+Policies}

Un'ampia directory web realizzata dal progetto SCAPE nel 2015 che elenca le politiche della conservazione digitale pubblicamente disponibili online per le biblioteche, gli archivi, i centri dati e enti vari.

\section{Practical Policy Recommendations}

https://www.rd-alliance.org/group/practical-policy-wg/outcomes/practical-policy

Elementi di politiche attuabili dalla Research Data Alliance, compresi esempi delle versioni leggibili a mano e a macchina.

\section{Casi di studio}

\section{A Digital Preservation Policy for Parliament} http://www.parliament.uk/documents/upload/digitalpreservationpolicy1.0.pdf

Lo scopo di questa policy, pubblicata nel 2009, è di affermare e comunicare i principi che guidano le attività del Parlamento britannico nel garantire la conservazione delle sue risorse informative digitali. I documenti strategici di alto livello, le procedure, gli standard e le guide, verranno realizzati in futuro per riferirsi agli aspetti specifici legati alla strategia. (17 pagine) 


\section{Hampshire Archives and Local Studies (HALS) Digital Preservation Policy}

http://documents.hants.gov.uk/archives/HRODigitalPreservationPolicy.pdf

Per far fronte al rischio di perdere risorse digitali, HALS ha creato una serie di strategie politiche di conservazione digitale. Le politiche delineano l'approccio adottato dal Record Office per la conservazione digitale; le strategie mirano, d'altra parte, a descrivere più nel dettaglio questo approccio, includendo le specifiche tecniche, ove opportuno.

\section{DPC case note: Cabinet papers - policy as a measure of commitment}

\section{http://www.dpconline.org/component/docman/doc download/449-casenotecabinetpapers.pdf}

Questo caso di studio del National Archives approfondisce l'analisi delle relazioni esistenti tra politiche e le pratiche nell'ambito della conservazione digitale. Le organizzazioni di supporto finanziario dovrebbero richiedere copie, dal momento che queste rappresentano l'impegno da parte dell'organizzazione a un accesso a lungo termine. II National Archives ha digitalizzato consistenti volumi dei Documenti di Governo britannico e ha attentamente tenuto in conto il sistema di politiche per una gestione delle risorse digitali a lungo termine. Maggio 2010 (3 pagine).

DPC case note: Welsh journals online: effective leadership for a common goal http://www.dpconline.org/component/docman/doc download/450-casenotewelshjournals.pdf Questo caso di studio finanziato da JISC ha analizzato il complesso progetto di digitalizzazione della National Library of Wales, un esempio di istituto in cui sono richiesti diversi stakeholder e diverse competenze. La nomina di un solo membro senior dello staff come incaricato principale della conservazione digitale e la concessione di lavorare all'interno di diversi settori dell'ente hanno fatto in modo che non ci fossero molti dubbi sull'assunzione di responsabilità per azioni legate alla conservazione. Giugno 2010 (3 pagine).

\section{British Library Digital preservation strategy 2013-2016}

\section{http://www.bl.uk/aboutus/stratpolprog/collectioncare/digitalpreservation/strategy/dpstrategy.ht}

\section{$\underline{\mathrm{ml}}$}

La strategia della British Library prevede quattro priorità, ognuna delle quali è seguita da una serie di azioni. Queste priorità sono allineate all'approccio generale della biblioteca riguardo alla collezione e ai suoi cinque principi di gestione sostenibile: predire, proteggere, dare priorità, conservare, e concedere.

Priorità Strategica 1: Assicurarsi che il proprio repository digitale riesca ad archiviare e conservare a lungo termine le collezioni

Priorità Strategica 2: Gestire i rischi e le sfide legate alla conservazione digitale durante tutto il ciclo di vita della collezione digitale

Priorità Strategica 3: Integrare la sostenibilità digitale come un principio organizzativo per la pianificazione della biblioteca digitale e del suo sviluppo

Priorità Strategica 4: Trarre vantaggio dalla collaborazione con altri enti nazionali e internazionali sulle iniziative legate alla conservazione

Per ognuna delle priorità si possono trovare maggiori dettagli nella versione completa disponibile al seguente pdf (16 pagine). 


\section{Wellcome Library's Preservation Policy}

http://wellcomelibrary.org/what-we-do/library-strategy-and-policy/preservation-policy/

II fine della strategia di conservazione della Wellcome Library è di offrire una spiegazione completa sulla conservazione delle collezioni della suddetta biblioteca, occupandosi di tutte le risorse in tutti i formati. La policy è costituita da tre parti che, a loro volta, comprendono una dichiarazione generale, la gestione delle risorse fisiche e la gestione di quelle digitali. (25 pagine).

\section{UK Data Archive Preservation Policy \\ http://data-archive.ac.uk/media/54776/ukda062-dps-preservationpolicy.pdf}

Questa policy è stata pubblicata nel 2014 e definisce i principi che sono alla base delle principali attività della UK Data Archive, la conservazione attiva delle risorse digitali per il loro utilizzo e riutilizzo all'interno di una specifica comunità di utenti. Da un punto di vista relativo alla conservazione, questa policy è generalmente in linea con il modello di riferimento OAIS, con alcune integrazioni e modifiche specifiche per le risorse che sono all'interno dell'Archivio. L'Archivio presenta una serie di requisiti vincolanti per le sue attività di conservazione digitale. Questi requisiti sono indicati all'interno della policy, così come vengono forniti ulteriori dettagli sulla modalità in cui questi ultimi possono essere soddisfatti in relazione a delle richieste normative, buone pratiche archivistiche, sicurezza delle informazioni e finanziamenti disponibili. (16 pagine).

\section{Digital Preservation Strategies for a Small Private College}

http://files.archivists.org/pubs/CampusCaseStudies/CASE-16-MegMiner-Final.pdf

II progetto POWRR (2011 - 2014) ha analizzato, valutato e raccomandato delle soluzioni scalabili di conservazione digitale per le biblioteche che dispongono di piccole quantità di dati e/o di poche risorse. Le 'buone pratiche' consolidate nella conservazione digitale si occupano in misura minore di ciò che concerne repository, i quali, a loro volta, non possono allocare fondi o personale flussi di lavoro della conservazione digitale. Meg Miner, dell'Università Wesleyan, Illinois, parla di cosa si può fare per garantire una buona gestione dei record nativi digitali o digitalizzati a livello istituzionale prima che venga messo in atto un sistema generale di conservazione. 2015 (13 pagine).

\section{University of Edinburgh - Developing a Digital Preservation Policy}

http://www.dpconline.org/component/docman/doc download/1321-making-progress-hsbc-nov-

\section{4-lee}

Un'ottima presentazione, e un caso di studio, da parte di Kirsty Lee presso l'Università di Edimburgo, durante un workshop della DPC 'Making Progress in Digital Preservation' a Ottobre del 2014, sulla metodologia da lei utilizzata per lo sviluppo di una policy di conservazione digitale a Edimburgo. (14 pagine) 


\section{Collaborazioni}

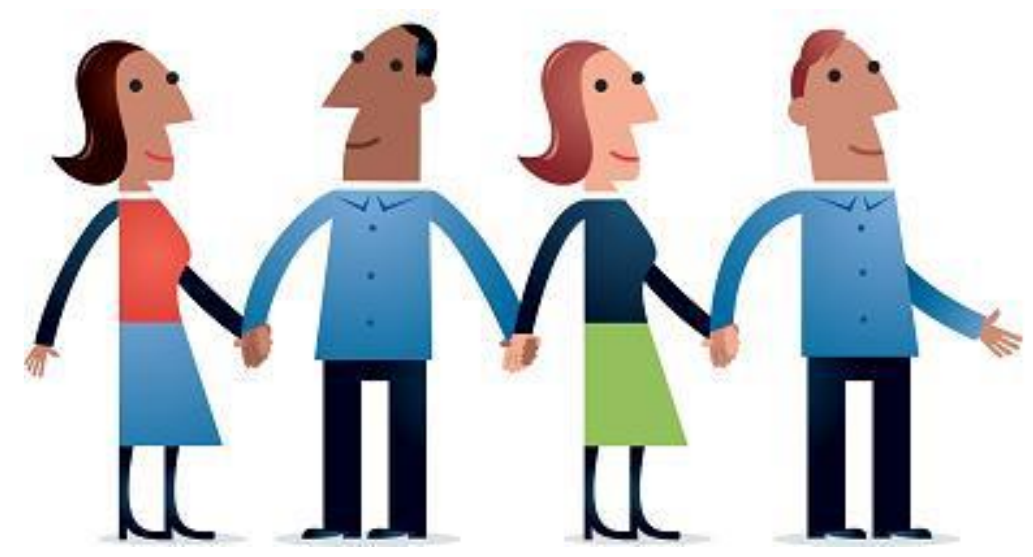

Illustrazione di Jørgen Stamp digitalbevaring.dk CC BY 2.5 Denmark

\section{Introduzione}

Esistono motivazioni stringenti e, in taluni casi, pressioni politiche, quando si vuole avviare una collaborazione all'interno e tra le organizzazioni per confrontarsi in maniera efficace e superare gli ostacoli relativi alla conservazione digitale. L'insieme di competenze richieste a tale scopo presuppone una flessibilità all'interno delle strutture organizzative che agevoli il lavoro nei gruppi interdisciplinari.

Esiste una considerevole sovrapposizione nelle problematiche legate alla conservazione digitale riscontrate da tutte le organizzazioni in tutti i settori, perciò ha senso mettere insieme competenze ed esperienze. La comunicazione con i principali stakeholder, utilizzando termini e un linguaggio comune a tutti sarà importante per la costruzione e il mantenimento delle collaborazioni.

\section{Collaborazioni interne}

Si è soliti pensare che le collaborazioni siano esterne. Tuttavia, nell'immediato futuro, la maggior parte delle biblioteche e degli archivi si troveranno a gestire una combinazione di risorse cartacee e digitali e avranno bisogno di preparare la loro organizzazione per gestire i bisogni delle due risorse. La sfumatura dei confini e i cambiamenti dei cicli di vita generati dalla conservazione digitale implicano che le sezioni e i comparti strutturalmente separati di una stessa organizzazione debbano cooperare al fine di integrare la conservazione e la gestione delle risorse digitali con altre risorse.

Questa cooperazione e lavoro congiunto potrebbe anche dimostrarsi impossibile a meno che non ci siano dei meccanismi messi in atto che possano fare in modo che ciò avvenga e non ci sia un chiaro consenso esecutivo e una sponsorizzazione dei decisori a promuovere l'attività. 
A livello strategico, una combinazione di commissioni con potere di sanzioni disciplinari e un gruppo di progetto incaricato dello sviluppo e supervisione degli obiettivi sono uno dei mezzi per poter assicurare che tutti i settori della stessa amministrazione collaborino. A livello operativo, bisognerà concentrarsi sul definire quali attività specifiche sono richieste e dove certe responsabilità di logica risiedono. Uno dei mezzi utilizzati è anche la creazione di gruppi di lavoro che analizzano situazioni specifiche. Altrettanto importanti saranno una buona comunicazione e promozione (si veda Sostegno).

Mentre la necessità di una politica o di una strategia riferita alla conservazione digitale potrebbe essere ben percepita all'interno dell'équipe di archiviazione, il motore verso una collaborazione interna è tipicamente una risposta a una problematica specifica che l'organizzazione ha di fronte a sé.

Vantaggi

- La collaborazione interna fa buon uso delle capacità disponibili e competenze, e riconosce che la conservazione digitale è un argomento istituzionale e non appartiene esclusivamente all'archiviazione.

- Promuove un lavoro tra vari team migliorando la comprensione di obiettivi comuni e delle persone coinvolte.

- Prima la conservazione digitale diventerà parte del lavoro quotidiano di un'organizzazione e dei suoi impiegati, meglio sarà per la loro transizione e preparazione al mondo sempre più digitale.

- Riconosce la varietà di capacità richieste per l'ambiente digitale in generale e per quello della conservazione digitale in particolare.

- Tende maggiormente ad essere mirato e conforme a finalità e priorità istituzionali.

- Mantiene un alto profilo per il tipo di lavoro.

\section{Svantaggi}

- Potrebbe diventare frustrante e richiedere un dispendio di tempo nel breve periodo.

- Inizialmente, la comunicazione potrebbe essere difficile - per esempio, ci sono alcune discussioni sulla terminologia legate al termine 'archivi' che assume significati differenti per archivisti e persone del settore IT.

- I dirigenti potrebbero non essere inclini a rischiare una perdita di controllo percepita.

- Le persone potrebbero non sentirsi a loro agio con nuove modalità di lavoro.

- Le strutture organizzative potrebbero non essere sufficientemente flessibili nell'agevolare collaborazioni effettive tra diverse sezioni.

\section{Collaborazioni esterne}

Diversi sarebbero i motivi per incentivare le collaborazioni esterne. Ad esempio, la volontà da parte di singoli specialisti di lavorare con altri colleghi professionisti e cercare una convalida esterna per le loro idee o per le strade intraprese. D'altra parte c'è il riscontro riferito alle opportunità di finanziamenti esterni, in un panorama in cui i finanziatori puntano molto sulla 
collaborazione. Alcuni esempi di tipi di collaborazione esterna nel settore della conservazione digitale sono:

- Collaborazione su un problema specifico per rendere più semplice e più gestibile l'avanzamento dei lavori. La Digital Preservation Coalition nel Regno Unito ne è un esempio. I membri sono incoraggiati a impegnarsi e collaborare su una serie di diverse situazioni legate alla conservazione digitale sia a livello alto che su argomenti specifici. Un altro esempio è la Section for Archives and Technology of the Archives and Records Association che unisce membri di organismi professionali per analizzare aspetti specifici del lavoro e condividere le basi delle attività correnti.

- Collaborazione su uno standard. Un esempio di questo tipo di collaborazione potrebbe essere la richiesta fatta nel 2015 di collaborazione su una revisione del modello di riferimento OAIS. In un'iniziativa coordinata dalla DPC, i professionisti che lavoravano in quel campo erano incoraggiati a impegnarsi e a inserirsi negli obiettivi comuni. (si veda http://wiki.dpconline.org/index.php?title=OAIS Community).

- Collaborazione su una parte specifica di un software o sistema. Un esempio di questa collaborazione potrebbero essere i gruppi di utenti che affrontano soluzioni software di conservazione digitale, sia commerciali che open source. Quando si scopre una parte di software per la prima volta, essere capaci di condividere le esperienze e imparare dagli altri è una cosa di immenso valore.

- Collaborazione entro un'area geografica precisa. Ci sono molti esempi di organizzazioni che collaborano in base alla loro vicinanza geografica e alla facilità che questo comporta nel lavorare insieme. Un esempio è the Digital Preservation Group

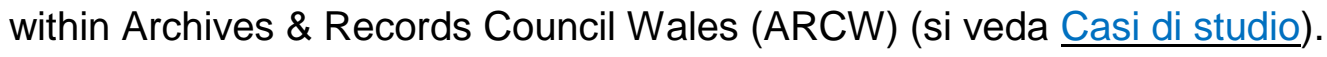

Vantaggi

- Impegno organizzativo e autorità.

- Accordi formali che garantiscono una precisa assegnazione di responsabilità tra i partner.

- Comprensione avanzata di problematiche complesse.

- Maggiori benefici pratici ottenuti dall'unione di risorse e competenze.

- Una reputazione migliore grazie alla realizzazione di successo di un progetto o per il fatto di essere in grado di gestire la conservazione digitale.

- Prospettive migliori per future collaborazioni reciprocamente vantaggiose.

\section{Svantaggi}

- Difficoltà nella determinazione di accordi chiari che possono essere accettati da tutte le parti.

- Tempo che si impiega nella decisione dei team o di un sistema di collaborazione.

- Difficoltà nella comunicazione tra i diversi contesti professionali e organizzativi.

- Limiti burocratici potenziali. 
La collaborazione esterna può avvenire su base informale o formale e tutti coloro che lavorano nel settore hanno sempre condiviso esperienze, e spesso la collaborazione informale costituisce parte dell'avanzamento continuativo professionale del singolo.

Le collaborazioni più grandi e complesse tendono a basarsi su un accordo formale di partenariato che potrebbe essere utile per definire la portata e i limiti della relazione di lavoro e per attribuire delle responsabilità precise.

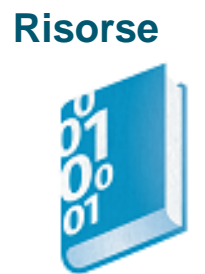

Benefits from Research Data Management in Universities for Industry and Not-for-Profit Research Partners

Esegue una mappatura degli stakeholder usando il KRDS Benefits Framework per analizzare i benefici della gestione dei dati associati alle collaborazioni di ricerca tra Accademia-Industria e Accademia-Organizzazioni No-Profit presso l'Università di Bath. Presenta ai diversi stakeholder un elenco riepilogativo dei vantaggi che possono provenire dalla gestione dei dati della ricerca e di conservazione in queste collaborazioni.

WWW

Aligning National Approaches to Digital Preservation Conference Proceedings 2012 http://educopia.org/publications/anadp

Questa pubblicazione contiene una raccolta di saggi revisionati realizzati dai panel e dai partecipanti alla conferenza. Ha come obiettivo quello di definire un insieme di punti sui quali avviare un processo di allineamento generale sulle iniziative riferite alla conservazione digitale, e ribadisce la necessità di collaborazioni strategiche internazionali per supportare la conservazione della nostra memoria culturale collettiva (342 pagine).

\section{North West Region Digital Preservation Group}

https://nwrdpg.wordpress.com/

II North West Region Digital Preservation Group è un esempio di collaborazione informale geografica che coinvolge autorità locali, accademici e archivisti professionisti. I risultati sono delle linee guida per i detentori, un workbook per gli archivisti e degli studi pilota sull'archiviazione web e sugli archivi di e-mail. 




DPC case note: Welsh journals online: effective leadership for a common goal https://www.dpconline.org/component/docman/doc download/450-casenotewelshjournals.pdf Questo caso di studio finanziato da JISC ha analizzato il complesso progetto di digitalizzazione della National Library del Wales, un esempio di istituto in cui sono richiesti diversi stakeholder e diverse competenze. La nomina di un solo membro senior dello staff come incaricato principale della conservazione digitale e la concessione di lavorare all'interno di diversi settori dell'ente hanno fatto sì che non ci fossero troppi dubbi sull'assunzione di responsabilità per azioni legate alla conservazione. Giugno 2010 (3 pagine).

\section{DPC case note: Freeze Frame preservation partnerships}

http://www.dpconline.org/component/docman/doc download/434-casenotefreezeframe.pdf

Questo caso valuta il rapporto che esiste tra il progetto di durata relativamente breve 'Freeze Frame' presso lo Scott Polar Research Institute e il repository istituzionale che aveva garantito dei servizi di conservazione a lungo termine per permettere un accesso alla fine del progetto. Lo studio dimostra che le piccole organizzazioni non hanno necessariamente bisogno di definire delle infrastrutture sofisticate di conservazione quando iniziano il processo di digitalizzazione. La collaborazione può recare vantaggi inaspettati ad entrambe le parti, ma deve essere attentamente gestita e documentata. Aprile 2010 (4 pagine).

\section{Community Action via UK LOCKSS Alliance}

http://www.slideshare.net/edinadocumentationofficer/ukla-dpc-final

Questa è la presentazione da parte di Adam Rusbridge alla Digital Preservation Coalition on Getting Started in Digital Preservation, il 28 Febbraio 2011. Si discute sul ruolo della LOCKSS Alliance britannica e sulla collaborazione nella conservazione delle riviste elettroniche.

\section{Archives \& Records Council Wales Digital Preservation Working Group \\ http://www.nationalarchives.gov.uk/documents/Cloud-Storage-casestudy Wales 2015.pdf}

Questo studio sul National Archives presenta l'esperienza di un gruppo di lavoro trasversale degli archivi Welsh che coopera per testare il dispiegamento di diversi servizi e sistemi per una verifica concettuale relativa all'archiviazione cloud. Chiarisce il contesto organizzativo, la diversa natura dei loro requisiti e gli approcci alla conservazione digitale e la loro esperienza nella selezione, utilizzo e verifica della conservazione digitale nel cloud. Gennaio 2015 (10 pagine). 
A collaborative infrastructure for permanent access to digital heritage in The Netherlands http://www.ncdd.nl/wp-content/uploads/2016/03/Network Digital Heritage Netherlands.pdf

Nel 2014 venne istituito, da parte di un gruppo di organizzazioni nazionali nei Paesi Bassi, il Network Digital Heritage (NDE). Questo network presentava una strategia per lo sviluppo di infrastrutture nazionali e multi dominio di servizi del patrimonio digitale. Uno dei programmi si concentra sulla conservazione digitale (patrimonio digitale sostenibile). Lo scopo di questo programma è di lavorare in un'ottica di condivisione trasversale, di utilizzo, e di aumento dei servizi per la conservazione sostenibile e per l'accesso, prestando attenzione ai costi di gestione e alle ripartizioni di responsabilità. Questo programma è portato avanti dalla NCDD, National Coalition for Digital Preservation. (3 pagine).

\section{The SPRUCE project}

http://wiki.opf-labs.org/display/SPR/Home

II progetto Sustainable PReservation Using Community Engagement (SPRUCE) (2011-2013) ha cercato di ispirare, guidare e far sì che HE, FE e altri enti culturali potessero colmare le lacune esistenti nella conservazione digitale e utilizzare la preparazione acquisita da questa attività riferita a un caso aziendale per la conservazione digitale. Questa collaborazione multiistituzionale ha unito archivisti ed esperti informatici mediante eventi di mashup e un hackaton. Tra i due risultati principali del progetto ci sono il Business Case Toolkit(http://wiki.dpconline.org/index.php?title=Digital Preservation Business Case kit) e COPTR (Community Owned digital Preservation Tools Registry) (si veda http://coptr.digipres.org/Main Page).

\section{Filling the Digital Preservation Gap Case Study \\ http://digital-archiving.blogspot.co.uk/2015/12/research-data-spring-case-study-for.html}

Una collaborazione tra le Università di Hull e York. Lo scopo del progetto era di colmare una lacuna esistente nelle infrastrutture di gestione di dati della ricerca sulla conservazione attiva dei dati. Sia Hull che York disponevano di repository digitali e di archiviazione sufficienti, ma non avevano, d'altra parte, sistemi e flussi di lavoro che potessero far fronte alla conservazione attiva dei dati. 


\section{Sostegno}

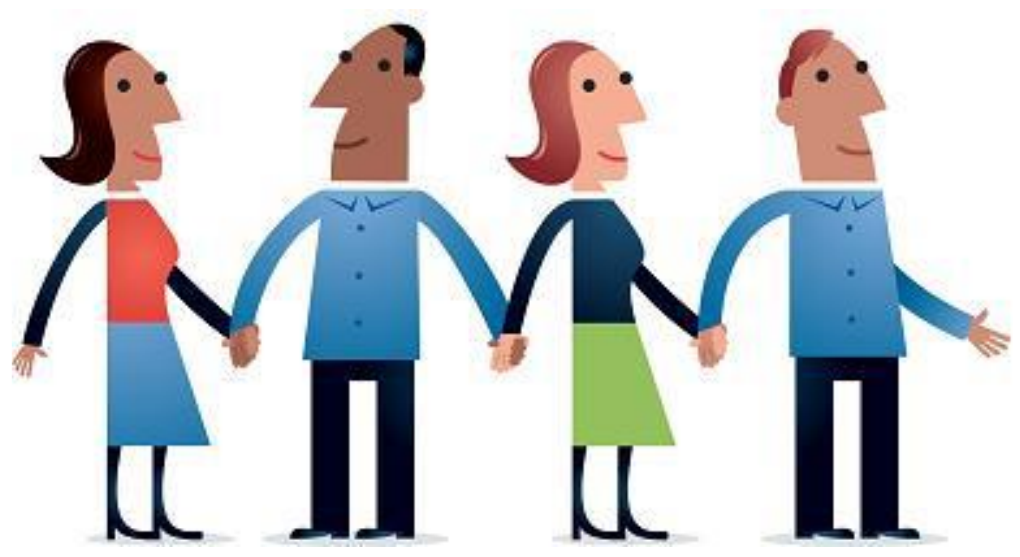

Illustrazione di Jørgen Stamp digitalbevaring.dk CC BY 2.5 Denmark

\section{Introduzione}

La conservazione digitale si basa su un insieme complesso di competenze e servizi, per cui i dirigenti della conservazione hanno bisogno di coordinare molteplici competenze, politiche, strumenti e servizi a partire da risorse disparate. Per alcune organizzazioni la conservazione digitale è una pratica completamente nuova e si troveranno a dover organizzare le risorse per la prima volta. Anche i programmi predefiniti si troveranno di fronte a nuove sfide e, quindi, anche l'insieme di strumenti e servizi richiesti potrebbe costantemente variare. Dunque, l'abilità di comunicare con lo staff, le strutture, e le organizzazioni è diventata una delle competenze principali per i responsabili della conservazione digitale di successo.

Dal momento che la tecnologia e il personale continuano a subire cambiamenti, la comunicazione e l'attività di sostegno devono rappresentare un percorso continuo anziché sporadico.

Nei primi periodi della conservazione digitale, la comunicazione e l'attività di sostegno non erano soliti usare mezze misure nell'affermazione dell'impatto sociale ed economico della perdita dei dati e dell'obsolescenza. Con l'arrivo di nuove soluzioni i messaggi sono diventati più velati.

L'attività di sostegno è diventata una pratica sempre più rivolta ad identificare gli stakeholder e ad aiutarli a capire:

- come le loro scelte facciano diventare le collezioni digitali più o meno resilienti;

- i benefici che possono derivare da una gestione attiva di risorse digitali ben strutturate e accessibili;

- la necessità dell'investimento - sia di tempo, denaro o altre risorse - e fino a dove è richiesto il raggiungimento di questi benefici. 
In un contesto istituzionale, ciò significa conoscere tutti gli agenti coinvolti nel ciclo di vita di un oggetto digitale, aiutarli a dare delle priorità e supportare le azioni che rendono e mantengono le collezioni solide, e scoraggiare quelle che mettono le collezioni a rischio.

\section{Analisi degli stakeholder}

L'analisi degli stakeholder inizia dal capire chiaramente le finalità della conservazione digitale di un'organizzazione prima di identificare gli stakeholder esterni e interni che possono influire su questi obiettivi. Dopo averle identificate è possibile sviluppare un piano che veicolerà le specifiche finalità per poi impiegarle nel processo di conservazione digitale. Portare avanti questo processo seguendo una chiara metodologia produrrà dei risultati migliori e coinciderà con una serie di altre attività di conservazione digitale come lo sviluppo di politiche e strategie (si veda Politiche e strategie istituzionali), realizzando uno studio della fattibilità (si veda Costi, benefici, impatti e studi di fattibilità) e identificando degli standard pertinenti e buone pratiche (si veda Standard e migliori pratiche).

I passaggi seguenti aiuteranno a facilitare un'analisi a tutto raggio degli stakeholder:

1. Identificare ciò a cui si aspira mediante le attività di conservazione digitale. Ciò implica esplicare le principali collezioni coinvolte e i principali obiettivi e finalità, così come $i$ benefici potenziali (si veda Studi di fattibilità, costi, benefici e impatti) che si raccoglieranno. Ciò fornirà un riferimento chiaro e un focus riferito alle attività di sostegno e si potrà poi adattare a diversi pubblici target.

2. Identificare i gruppi e le persone che possono inibire o consentire le attività di conservazione digitale. Questi possono essere interni o esterni all'organizzazione, e ciascuno degli stakeholder può assumere diversi ruoli. Ad esempio, si potrebbe identificare il personale dell'area informatica come un gruppo principale che poi potrebbe a sua volta includere un dirigente dei servizi IT, programmatori e personale di supporto. Alcuni di questi potrebbero essere facilmente reperibili all'interno della propria struttura, altri invece far parte delle strutture di dirigenza, e alcune figure professionali essere completamente esterne. Ciò significa che si potrebbe aver bisogno di includere altri dirigenti o proprietari di servizi all'interno della propria analisi di stakeholder.

3. Strutturare i gruppi di stakeholder e le singole persone in pubblico di riferimento che incidono sugli obiettivi e sulle priorità. II pubblico selezionato probabilmente rispecchierà i metodi professionali della propria organizzazione e/o l'approccio alla conservazione digitale, facendo forse riferimento a parti specifiche della struttura organizzativa (ad es., Dirigenza, IT, Responsabili dell'informazione) o attraverso il suo ruolo in riferimento al processo di conservazione digitale (ad es., Finanziatori, Detentori, Utenti).

4. Stabilire delle relazioni di collaborazione solide con $i$ principali gruppi di stakeholder che si sono identificati al fine di sostenere il percorso verso le finalità decise nello Step 1. Capire i bisogni, le priorità e i limiti degli stakeholder interni o esterni potrebbe dare precedenza alle informazioni che danno evidenza direttamente dei propri progetti e migliorano la propria consapevolezza rispetto a cosa gli stakeholder vogliono dalle attività di conservazione digitale. Gli stakeholder potrebbero essere ostacolati dal budget e/o dai limiti legislativi di cui bisognerà essere consapevoli. D'altra parte 
potrebbero avere un tipo di competenze rilevanti o risorse che possono essere impiegate nelle attività di conservazione digitale. Inoltre, capire il linguaggio e la terminologia che usano gli stakeholder potenzia le strategie di comunicazione efficaci e può aiutare ad evitare delle difficoltà che emergono quando gli stakeholder comprendono un termine o un concetto in modi diversi. Generalmente, la capacità di utilizzare il linguaggio degli stakeholder fa sì che i colleghi e collaboratori accettino i progetti di riferimento. Se gli stakeholder hanno degli interessi contrastanti, allora bisogna fare da mediatori.

5. Investire sull'impegno reciproco, definire in modo chiaro quale informazione condividere con questi gruppi aiuterà ad assicurare il loro coinvolgimento. Ciò dovrebbe includere:

- Messaggi chiave basati sulle proprie finalità e obiettivi. Dovrebbero essere delle affermazioni semplici e dirette scritte in un linguaggio chiaro per far sì che possano essere comprese da un elevato numero di utenti non specialisti. Dovrebbero poi idealmente essere allineati con strategie e finalità più ad ampio raggio.

- I benefici e le convinzioni che gli stakeholder maturano dalla partecipazione/supporto alle attività di conservazione digitale. Ad esempio, un dirigente IT potrebbe voler ridurre i costi dell'archiviazione cancellando o deduplicando archivi in esubero. Una chiara strategia di conservazione digitale può aiutare gli stakeholder a ridurre le loro richieste di archiviazione distinguendo le collezioni che devono essere mantenute da quelle che non sono più richieste.

- Cosa sarà loro richiesto per assicurare il successo. Per esempio, si potrebbero avanzare delle richieste di metadati chiari per i detentori o volere delle stime per il proprio reparto IT riferite alle quantità di archivi e larghezza di banda che saranno necessarie e su quando lo saranno.

- Quali limiti/pregiudizi sulla conservazione digitale si potrebbero dover affrontare. Ad esempio, la conservazione viene spesso confusa con il fatto di disporre solamente delle copie di backup. Ci si potrebbe trovare a dover adattare linguaggi e terminologia a specifici pubblici. Per esempio, alcuni termini, come 'archiviazione', hanno significati diversi in altri settori come l'IT.

6. Realizzare un piano per coinvolgere ciascun gruppo di stakeholder basandosi sulla propria conoscenza delle loro priorità, competenze e limiti, e utilizzando i vari messaggi precedentemente identificati. Si potrebbero dover usare diverse metodologie per i vari gruppi, personalizzando la propria modalità di comunicazione per riuscire ad adattarsi al meglio al pubblico e ai messaggi da trasmettere. Ciò potrebbe includere una serie di canali di comunicazione, tra cui presentazioni, documenti informativi e gruppi di lavori di stakeholder, così come sviluppare una serie di progetti e risorse come studi di fattibilità (si veda Studi di fattibilità, costi, benefici e impatti), politiche (si veda Politiche e strategie istituzionali), e registri dei rischi (si veda Gestione dei rischi e dei cambiamenti). 
Conservazione digitale nel mondo dei media

Stranamente la conservazione digitale non riceve molte attenzioni dai principali mezzi di comunicazione. Tra i clichés relativi alla conservazione digitale si trovano le terribili storie sulla perdita dei dati e sull'incombente 'epoca buia digitale' o affermazioni stereotipate sull'archivio indistruttibile.

Ma la realtà dei fatti è molto più banale e impercettibile. Non si presta molta attenzione alle esigenze pratiche, specifiche e facilmente raggiungibili che forniscono un accesso a lungo termine, come quelle descritte in questo Manuale, ignorando che possono portare dei veri benefici alle istituzioni e alle loro comunità di utenti finali.

In alcuni contesti di attività di sostegno potrebbe essere utile riferirsi a un vocabolario comune per supportare la spiegazione di termini e concetti nella conservazione digitale. Alcuni esempi sono riportati nella sezione seguente delle Risorse.

La grande comunità della conservazione digitale ha realizzato dei corti d'animazione per le attività di sostegno come quelli selezionati di seguito in Risorse. Sono corti, d'intrattenimento, e spesso sono un tramite per dei messaggi importanti sulla conservazione digitale verso il grande pubblico dei non esperti.

\section{Risorse}



Team di conservazione digitale e disastro nucleare: un cartone animato https://www.youtube.com/watch?v=pbBa6Oam7-w

Un cartone animato divertente sull'importanza dei repository digitali affidabili, dei metadati e dei mezzi di informazione digitali aggiornati (3 minuti 18 secondi).

Team di conservazione digitale e il disastro dell'aeroplano

https://www.youtube.com/watch?v=EKnsZZzuUr4

Un cartone animato divertente che descrive gli effetti dell'obsolescenza e l'importanza del processo di migrazione (3 minuti 37 secondi).

Team di conservazione digitale e l'avventura artica

https://www.youtube.com/watch?v=PGFOZLecjTc

Un cartone animato divertente sull'importanza del piano di conservazione (4 minuti 22 secondi).

Team di conservazione digitale e il fatale dilemma criptico

https://www.youtube.com/watch?v=Yun9hkPPF9M

Un cartone animato divertente sull'importanza della rappresentazione delle informazioni (4 minuti 9 secondi). 
Increasing Participation in Internal RDM Training Sessions

http://www.dcc.ac.uk/resources/developing-rdm-services/increasing-participation-training

Questo caso di studio riguarda gli approcci adottati dai due progetti Jisc MRD per garantire un buon livello di partecipazione alle loro sessioni di formazione sulla gestione dei dati della ricerca (RDM). 2013 (4 pagine).

Defining and Formalizing a Procedure for Archiving the Digital Version of the Schedule of Classes at the University of Michigan

http://files.archivists.org/pubs/CampusCaseStudies/Case2Final.pdf

Nancy Deromedi dell'Università del Michigan descrive la formazione di una collaborazione con un'unità amministrativa principale che fino a quella data non era stata partner attivo all'interno dell'università, e la sensibilizzazione sugli aspetti archivistici nel momento in cui l'unità transitava da un sistema ibrido (digitale e analogico) a un processo esclusivamente digitale. (8 pagine). 


\section{Acquisizione e servizi di parti terze}



Illustrazione di Jørgen Stamp digitalbevaring.dk CC BY 2.5 Denmark

\section{Introduzione}

In questa sezione si presenteranno gli aspetti principali e le linee guida per la selezione e utilizzo di servizi di parti terze per la conservazione digitale. I modi in cui si può acquisire un servizio spesso variano a seconda del settore o del Paese. Le singole organizzazioni devono identificare e seguire le loro politiche d'acquisto in conformità alle disposizioni legali e normative in vigore per garantire che i servizi siano acquistati utilizzando le corrette procedure.

Vengono messe a disposizione tre tabelle come parte della guida: Personale per le attività di acquisizione; Questioni e potenziali vantaggi e svantaggi nell'utilizzo dei servizi di parti terze nelle attività di conservazione digitale; e una Lista di controllo per la valutazione della preparazione all'archiviazione per la conservazione digitale poiché l'acquisizione rappresenta solitamente un elemento chiave per implementare l'archiviazione (si veda Archiviazione e https://www.dpconline.org/handbook/technical-solutions-and-tools/cloud-services). La sezione

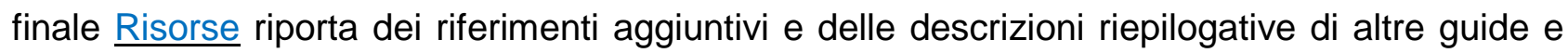
casi studio.

Sarà prestata molta attenzione, ovviamente, ai costi nel decidere se esternalizzare o meno la conservazione digitale, ma ci sono anche altri fattori da tenere in considerazione nonché i vantaggi e gli svantaggi di ciascuno che dovranno essere bilanciati rispetto alla missione generale dell'ente. Questi fattori includono il contratto, l'accordo sul livello dei servizi, le funzionalità e le qualità dei servizi offerti, l'integrazione con i processi e l'ambiente dell'ente, il ripristino di emergenze e il piano di continuità operativa, la capacità di interrompere il servizio se necessario, e la modalità con la quale il servizio possa essere monitorato e misurato. Per esempio, i requisiti legali per la privacy dei dati o la confidenzialità potrebbero influire sul fatto 
che l'esternalizzazione sia o non sia appropriata data la competenza del fornitore del servizio e su dove si trova fisicamente il servizio.

Esternalizzare compiti o servizi specifici non rappresenta affatto un fenomeno nuovo. I repository hanno esternalizzato per decenni alcune delle loro operazioni. Questo è infatti un ambito in cui le lezioni apprese dall'esternalizzazione in altri servizi possono avere un peso. Uno dei principali insegnamenti che può essere applicato direttamente all'ambito digitale è il fatto importante di disporre di risorse umane sufficienti e di possedere una conoscenza informatica per essere in grado di preparare delle specifiche efficaci.

\section{II personale per procedure di acquisizione}

La misura in cui i potenziali vantaggi dell'utilizzo di servizi di parti terze possano essere massimizzati, minimizzando gli svantaggi, dipenderà in maniera decisiva dalla quantità di risorse umane disponibili per le seguenti attività:

Personale per incarichi professionali

Definire i compiti organizzativi e una corretta gestione quando si selezionano i servizi di parti terze:

- Sostenere il concetto di conservazione digitale.

- Coinvolgere stakeholder interni adeguati.

- Sviluppare una strategia comunicativa non solo per il proprio gruppo d'acquisizione, ma per il più ampio gruppo di stakeholder.

- Mantenere un registro dei rischi aggiornato per le procedure di acquisizione.

- Utilizzare le competenze proprie dell'organizzazione: ad esempio, c'è una sezione specifica per l'acquisizione di beni e servizi?

Definire le competenze organizzative e amministrative adeguate nella selezione dei servizi di parti terze:

- Allineare i casi aziendali a specifiche necessità all'interno dell'organizzazione. Ciò potrebbe richiedere del tempo, ma è fondamentale per ottenere un buon risultato.

- Creare un ambiente in cui gli stakeholder possano contribuire al discorso e percepire che hanno apportato un contributo.

- Imparare dalle esperienze degli altri nella comunità di conservazione digitale: ciò potrebbe includere visite ai siti di riferimento e/o condivisione di documenti e osservazioni.

- Definire quali funzioni di conservazione debbano essere inserite, es., ingest; archiviazione, piano di conservazione, trattamento e attività di gestione. Tutte queste attività devono essere esternalizzate a fornitori di servizi terzi o solo in parte?

- Distinguere tra funzionalità essenziali e funzionalità a "valore aggiunto".

- Definire richieste non ambigue e quantificabili che possono essere utilizzate per verificarne la loro soddisfazione con il contraente. 
Chiarire le richieste legali:

- Seguire richieste e processi d'acquisizione istituzionali e normativi.

- Devono essere tenuti in considerazione la protezione dei Dati, la FOI, e altro contenuto sensibile o soggetto a copyright.

- Vale la pena dedicare del tempo in anticipo alla negoziazione dei propri contatti e accordi con i fornitori di parti terze. Le incomprensioni sono uno spreco di tempo.

- Se sono necessarie delle modifiche nel contratto o accordo proposto, è fondamentale definirle prima della sottoscrizione e accertarsi della sua validità legale definendo eventualmente delle clausole di riesame.

- Assicurarsi della presenza della clausola di non acquisizione obbligatoria.

- Assicurarsi della possibilità di rescissione senza clausole vessatorie ma con la semplice giustificazione dell'inadempienza contrattuale con l'obbligo di corresponsione solo del maturato.

- Capire in modo chiaro quali servizi e prodotti si stanno acquistando e quali sono quelli suscettibili di futuri aumenti di costo.

- Rendere disponibili ai contraenti tutte le clausole contrattuali compresa quella relativa al foro competente.

Mantenere una buona comunicazione tra il contraente e l'ente:

- Accordo sul livello dei servizi per identificare ruoli e responsabilità di ogni parte.

- Accesso solo alle infrastrutture tecnologiche o anche l'utilizzo di personale esterno /supporto allo sviluppo?

- In questo contesto sono anche importanti le capacità di creare dei rapporti più flessibili con i venditori.

- Esiste una comunità di utenti attivi per il sistema scelto che fornisce dei riscontri e una buona forma di interazione con il contraente?

Effettuare controlli di garanzia di qualità

- Stabilire rispettivamente responsabilità e funzioni come ad esempio controllo dell'integrità.

- Far coincidere i controlli di garanzia di qualità con le richieste quantificabili propriamente specificate nel contratto e assicurare che il fornitore stia soddisfacendo le richieste o cambiando/correggendo le loro pratiche per poterle soddisfare.

- Audit/conformità con le responsabilità legali.

Sviluppare e monitorare il contratto:

- Ciò potrebbe sembrare prematuro, ma una strategia d'uscita dovrebbe essere individuata in anticipo. La funzione della conservazione digitale sopravvivrà ai fornitori di servizi commerciali e infrastrutture tecnologiche correnti.

- Essere attenti al mercato e ai modelli finanziari usati dai venditori. Si potrebbe dover pensare fuori dagli schemi poiché questi modelli potrebbero non corrispondere con il modello finanziario prevalente nella propria organizzazione (spesa in conto capitale vs entrate/spese è un dilemma frequente).

- Consapevolezza di qualsiasi cambiamento dell'ambiente tecnologico per i fornitori di parti terze.

- Tenersi aggiornati con il mercato dopo che si sono concluse le proprie acquisizioni. Ė importante conoscere l'affidabilità in termini commerciali del proprio venditore. Aggiornare periodicamente la verifica della dovuta diligenza.

Questi costi dovranno essere aggiunti ai costi di contratto totali nel momento in cui si calcoleranno i costi-benefici nell'utilizzo dei servizi di parti terze per la conservazione digitale, 
tenendo a mente che la maggior parte di questi costi sarà o dovrebbe essere sostenuta anche se la conservazione non viene esternalizzata.

Aspetti e potenziali vantaggi e svantaggi nell'utilizzo di servizi di parti terze nelle attività di conservazione digitale

\begin{tabular}{|c|c|c|}
\hline Problematica & $\begin{array}{l}\text { Vantaggio potenziale } \\
\text { nell'uso di servizi di parti } \\
\text { terze }\end{array}$ & $\begin{array}{l}\text { Svantaggio potenziale nell'uso di } \\
\text { servizi di parti terze }\end{array}$ \\
\hline $\begin{array}{l}\text { Personale, competenze ed } \\
\text { esperienze limitati }\end{array}$ & $\begin{array}{l}\text { - Garantisce delle } \\
\text { competenze } \\
\text { specialistiche ed } \\
\text { esperienze che } \\
\text { potrebbero non essere } \\
\text { disponibili all'interno } \\
\text { dell'organizzazione. }\end{array}$ & $\begin{array}{l}\text { - Senza avere qualche } \\
\text { esperienza pratica e } \\
\text { competenza sarà difficile } \\
\text { stipulare e monitorare dei } \\
\text { contratti efficaci, così come } \\
\text { sarà complicato capire e } \\
\text { comunicare in modo efficace } \\
\text { le richieste } \\
\text { dell'organizzazione (o } \\
\text { valutare se queste siano } \\
\text { tecnicamente praticabili o } \\
\text { no). }\end{array}$ \\
\hline Costi & $\begin{array}{l}\text { - Fa sì che non si } \\
\text { debbano sviluppare } \\
\text { infrastrutture costose } \\
\text { (aspetto rilevante per } \\
\text { le piccole istituzioni). } \\
\text { - Se vi sono economie } \\
\text { di scala, esternalizzare } \\
\text { potrebbe essere meno } \\
\text { dispendioso. }\end{array}$ & $\begin{array}{l}\text { - L'analisi comparativa non è } \\
\text { molto definita, si tratta } \\
\text { tuttavia di un'area nuova. } \\
\text { - Rischio di fallimento } \\
\text { aziendale. } \\
\text { - Fino a che il mercato evolve } \\
\text { si può dipendere } \\
\text { eccessivamente da un solo } \\
\text { contraente. }\end{array}$ \\
\hline Velocità d'implementazione & $\begin{array}{l}\text { - Fa sì che si possa } \\
\text { agire nel breve e } \\
\text { medio termine, in } \\
\text { attesa di sviluppi } \\
\text { dell'infrastruttura. }\end{array}$ & $\begin{array}{l}\text { A meno che non ci siano } \\
\text { delle strategie di uscita } \\
\text { adeguate, si potrebbe } \\
\text { rimanere vincolati da un } \\
\text { contratto di } \\
\text { esternalizzazione più lungo } \\
\text { del previsto. }\end{array}$ \\
\hline Principali competenze & $\begin{array}{l}\text { - Permette all'istituzione } \\
\text { di concentrarsi su altri } \\
\text { aspetti della fornitura } \\
\text { di servizi. }\end{array}$ & $\begin{array}{l}\text { Pericolo sia di non } \\
\text { sviluppare sia di perdere } \\
\text { delle competenze di base e } \\
\text { specialistiche. C'è ancora } \\
\text { bisogno di saper prendere } \\
\text { decisioni consapevoli. }\end{array}$ \\
\hline
\end{tabular}




\begin{tabular}{|c|c|c|}
\hline $\begin{array}{l}\text { Considerazioni } \\
\text { sull'accesso }\end{array}$ & $\begin{array}{l}\text { - Il monitoraggio } \\
\text { dell'utilizzo potrebbe } \\
\text { essere più efficiente } \\
\text { (partendo dall'assunto } \\
\text { che il contraente è } \\
\text { capace di fornire delle } \\
\text { significative statistiche } \\
\text { sull'utilizzo). } \\
\text { - } \quad \text { i potrebbero essere } \\
\text { delle sinergie e dei } \\
\text { risparmi di spesa } \\
\text { nell'esternalizzazione } \\
\text { sia di accessi che di } \\
\text { conservazione. }\end{array}$ & $\begin{array}{l}\text { Difficoltà nel controllare i } \\
\text { tempi di risposta che } \\
\text { potrebbero a loro volta } \\
\text { essere inaccettabilmente } \\
\text { ridotti e/o più dispendiosi, } \\
\text { soprattutto per alcuni oggetti } \\
\text { ad alto uso. } \\
\text { - Potrebbe essere difficile } \\
\text { prevedere delle esigenze } \\
\text { future in questo ambito. }\end{array}$ \\
\hline Gestione dei diritti & $\begin{array}{l}\text { - Evita ciò che spesso } \\
\text { per l'istituzione } \\
\text { rappresenta un } \\
\text { elevato impiego di } \\
\text { risorse. }\end{array}$ & $\begin{array}{l}\text { Potrebbe aumentare in } \\
\text { modo significativo il costo } \\
\text { del contratto e/o di } \\
\text { negoziazioni complicate con } \\
\text { i detentori di diritti di parti } \\
\text { terze. }\end{array}$ \\
\hline Sicurezza & $\begin{array}{l}\text { - Il contratto può } \\
\text { garantire degli accordi } \\
\text { di sicurezza richiesti } \\
\text { dall'istituzione. }\end{array}$ & $\begin{array}{l}\text { Perdita di controllo, } \\
\text { soprattutto per risorse } \\
\text { riservate. }\end{array}$ \\
\hline Controllo qualità & $\begin{array}{l}\text { - Verrà redatto un } \\
\text { contratto inattaccabile } \\
\text { sulla base di stringenti } \\
\text { richieste di controllo } \\
\text { qualità. }\end{array}$ & $\begin{array}{l}\text { Rischio di perdita o } \\
\text { distorsione che potrebbe } \\
\text { essere inaccettabilmente } \\
\text { elevato per risorse } \\
\text { altamente importanti e/o } \\
\text { riservate. }\end{array}$ \\
\hline Archiviazione & $\begin{array}{l}\text { - Accedere a } \\
\text { disposizioni di } \\
\text { archiviazione } \\
\text { conosciute e gestite } \\
\text { professionalmente con } \\
\text { una facile replicazione } \\
\text { del contenuto e } \\
\text { controllo dell'integrità. }\end{array}$ & $\begin{array}{l}\text { - Questioni legate alla fiducia } \\
\text { e a considerazioni legali } \\
\text { quando si archiviano dati } \\
\text { sensibili. } \\
\text { - Difficoltà nell'anticipare i } \\
\text { costi effettivi di alcuni servizi } \\
\text { come il cloud storage e } \\
\text { computing, perché spesso } \\
\text { l'organizzazione non sa } \\
\text { esattamente di quanti servizi } \\
\text { ha bisogno. A questo livello } \\
\text { di incertezza si può ovviare } \\
\text { con l'esperienza. }\end{array}$ \\
\hline
\end{tabular}




\section{Liste di controllo per la selezione e il confronto di fornitori di servizi}

Le liste di controllo e gli standard possono rappresentare dei preziosi punti di partenza nella considerazione e valutazione dell'uso dei servizi di parti terze trattandosi di liste già elaborate che possono essere facilmente adottate o adattate per rispondere alle esigenze di ciascuno. In particolare, le liste di controllo supporteranno l'identificazione di ciò che si potrebbe dimenticare di tenere in conto così da indicare chiaramente questioni e necessità.

Le liste di controllo sono efficaci quando vengono associate a un modello di maturità. Ad esempio, i livelli di conservazione NDSA fanno in modo che le liste di controllo siano create per verificare quanto bene un fornitore svolga le attività per ogni livello.

Un'organizzazione identifica di quale livello di maturità necessita sia allo stato attuale che in futuro e poi cerca dei fornitori di servizi con livelli allineabili.

Le liste di controllo e gli standard per i servizi di repository sono punti di partenza importanti perché si possono selezionare e scegliere le parti delle liste di controllo che si adatterebbero a specifici servizi d'interesse. Esempi di liste di controllo e standard sono disponibili nella sezione Risorse, e se ne discuterà più nel dettaglio nella sezione del Manuale Audit e certificazione.

Si riporta qui di seguito una lista di controllo del Manuale per valutare la preparazione di archiviazione per la conservazione digitale:

Liste di controllo: domande per i propri fornitori di servizi relativi all'archiviazione e alla conservazione

$\square$ Qual è il livello di ridondanza dato dal sistema di archiviazione? In quante postazioni, fisicamente parlando, vengono tenute le risorse digitali? Qual è la distanza geografica tra queste ultime?

Esistono diversi tipi di tecnologia di archiviazione utilizzati per mitigare/diffondere il rischio? Per esempio archiviazioni online e off-line.

$\square$ Se un file è stato danneggiato o involontariamente alterato, come lo si può rilevare e quando avviene questa rilevazione? Le tracce di controllo o altre forme di registrazione dimostrano se sono stati effettuati controlli d'integrità dei dati e mostrano i risultati?

- Qual è la strategia di Disaster Recovery (DR), ad esempio, se un sistema di archiviazione non funziona, oppure ci si ritrova di fronte a una catastrofe naturale nel luogo in cui si trova l'archiviazione, come vengono recuperate le risorse digitali? Qual è stata l'ultima volta che questa strategia DR è stata testata?

- Qual è la strategia di migrazione di archiviazione quando si parla di obsolescenza tecnica? Cosa succede quando un sistema arriva alla fine del ciclo di vita e il contenuto ha bisogno di essere migrato verso un nuovo sistema? II contenuto rimane comunque accessibile durante questo processo? 


\begin{tabular}{|l|}
\hline$\square$ Qual è la strategia di uscita quando si usa un determinato tipo di archiviazione (ad es., in \\
loco, cloud), ad esempio cosa succede se un fornitore del sistema di archiviazione \\
fallisce?
\end{tabular}

\section{Risorse}



OAIS: Open Archival Information Systems: Reference Model for an Open Archival Information System. Recommended practice

https://public.ccsds.org/pubs/650x0m2.pdf

Offre una terminologia condivisa molto utile e un modello funzionale nell'identificazione dei requisiti per acquisire servizi di conservazione digitale da parti terze. (135 pagine).

\section{Core Trust Seal}

https://www.coretrustseal.org/

II Core Trust Seal rappresenta il primo passaggio, in un contesto di riferimento su scala globale, per la certificazione di repository affidabili. Questa valutazione del repository è costituita da 16 punti e può essere utilizzata come strumento di autovalutazione o revisione. 
IS016363: 2012 Audit and certification of trustworthy digital repositories http://www.iso16363.org/

ISO 16363 è un audit framework basato sulle evidenze per la conservazione digitale e consiste in più di 80 criteri che possono essere utilizzati per un'autoverifica o verifica esterna. I criteri usati nello standard sono riferiti all'intera organizzazione e non soltanto al sistema tecnico in cui il contenuto della collezione è archiviato. La versione pre stampa del Libro CCSDS Magenta dello standard è gratuitamente accessibile allindirizzo http://public.ccsds.org/publications/archive/652x0m1.pdf.

DIN 31644 Information and documentation - Criteria for trustworthy digital archives http://files.dnb.de/nestor/materialien/nestor mat 17 eng.pdf

La versione estesa del processo di autovalutazione degli archivi digitali è una lista utile che è stata realizzata dai NESTOR ${ }^{21}$ sulla base di DIN 31644 Information and documentation - Criteria for trustworthy digital archives standard (44 pagine).

The NDSA Levels of Digital Preservation: An Explanation and Uses

http://www.digitalpreservation.gov/ndsa/working groups/documents/NDSA Levels Archiving 2013.pdf

I Livelli di Conservazione della US National Digital Stewardship Alliance (NDSA) sono ampiamente utilizzati allinterno del Manuale e risultano utili per diverse aree della conservazione digitale. AVPreserve ha fornito dei mapping riferiti ai livelli di conservazione della NDSA con i profili di venditori dell'archiviazione cloud. (7 pagine).

\section{Where to keep research data DCC Checklist for Evaluating Data Repositories http://www.dcc.ac.uk/sites/default/files/documents/publications/Where\%20to\%20keep\%20rese arch\%20data.pdf}

Una lista di supporto della Digital Curation Centre su dove mantenere i dati al sicuro, include i livelli di maturità Service Level Agreement. Si basa principalmente sui repository esterni di parti terze che offrono un tipo di servizio gestito alla comunità di ricerca britannica. (20 pagine).

\section{The National Archives Cloud Storage Guidance}

http://www.nationalarchives.gov.uk/archives-sector/digital-collections.htm

Offre informazioni sull'acquisizione nei contesti di cloud computing per scopi relativi alla conservazione, compresi i casi di studio di diversi enti (si veda in basso). È nota per le sue considerazioni sugli aspetti legali.

21 Network of Expertise in Long-Term Storage of Digital Resources 
DPC procuring preservation event

http://www.dpconline.org/events/previous-events/1150-procuring-preservation-writing-andunderstanding-requirements-in-digital-preservation

Per una panoramica su alcuni degli elementi di requisiti di individuazione degli obiettivi si vedano le presentazioni nell'elenco, Requirements analysis, e Procuring Preservation: hoops, hurdles and processes sono tra le più rilevanti.

\section{Casi di studio}

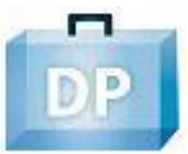

Archives \& Records Council Wales Digital Preservation Working Group

http://www.nationalarchives.gov.uk/documents/Cloud-Storage-casestudy Wales 2015.pdf

Questo studio sul National Archives presenta l'esperienza di un gruppo di lavoro trasversale degli archivi Welsh che coopera per testare il dispiegamento di una serie di servizi e sistemi per una verifica concettuale per l'archiviazione cloud. Chiarisce il contesto organizzativo, la diversa natura dei loro requisiti e approcci di conservazione digitale, e la loro esperienza nella selezione, utilizzo e verifica della conservazione digitale nel cloud. II caso di studio ha analizzato il software open source Archivematica con Microsoft Windows Azure; Archivematica con CloudSigma; Preservica Cloud Edition e ha iniziato a testare Archivematica con Arkivum 100. Gennaio 2015 (10 pagine).

\section{Tate Gallery}

http://www.nationalarchives.gov.uk/documents/Cloud-Storage-

\section{casestudy Tate Gallery 2015.pdf}

Questo studio presenta l'esperienza relativa allo sviluppo di un archivio digitale condiviso da quattro postazioni fisiche della Tate alimentate dal sistema di archiviazione commerciale Arkivum. Spiega il contesto organizzativo, la natura dei loro requisiti di conservazione digitale e gli approcci, e la loro motivazione circa la scelta di Arkivum come soluzione in loco, "Arkivum/OnSite", rispetto ad altre proposte basate sul cloud. Si conclude con una lezione chiave, e discute di piani per futuri sviluppi. Gennaio 2015 (7 pagine).

\section{Dorset History Centre}

http://www.nationalarchives.gov.uk/documents/Cloud-Storage-case-

study Dorset $2015 \% 281 \% 29$.pdf

Questo caso di studio si riferisce al Dorset History Centre, un servizio archivistico locale governativo. Chiarisce il contesto organizzativo dell'archivio, la natura dei suoi requisiti di 
conservazione e gli approcci, il suo progetto pilota di due anni con l'utilizzo di Preservica Cloud Edition (un servizio di conservazione digitale basato sul cloud), il caso aziendale e il finanziamento per il progetto pilota. Si conclude con una lezione importante appresa e sui piani futuri. Gennaio 2015 (9 pagine).

\section{Parliamentary Archives}

http://www.nationalarchives.gov.uk/documents/Cloud-Storage-

\section{casestudy Parliament 2015.pdf}

Questo caso di studio si riferisce agli Archivi Parlamentari e alla loro esperienza relativa all'attività di acquisizione mediante il sistema G-Cloud. Per una resilienza extra/strategia d'uscita hanno scelto due fornitori di servizi cloud con diverse infrastrutture sottostanti di archiviazione. Questo è un esempio di un archivio che utilizza un sistema ibrido di soluzioni di archiviazione (una parte cloud pubblica, e una parte installata localmente) per la conservazione digitale visto che l'archivio ha un sistema di conservazione installato localmente (Preservica Enterprise Edition) che è integrato con un archivio cloud e locale e archivia risorse confidenziali localmente, non nel cloud. Gennaio 2015 (6 pagine).

Partnering with IT to Identify a Commercial Tools for Capturing Archival E-mail of University Executives at the University of Michigan

http://files.archivists.org/pubs/CampusCaseStudies/CASE-14-FINAL.pdf

Aprille Cooke McKay, Bentley Historical Library, University of Michigan, analizzano le sfide e le opportunità del partenariato con IT per le problematiche legate alla Request for Proposal (RFP) per i software di archiviazione di e-mail commerciali. 2013 (53 pagine).

\section{University of Sheffield Procurement Case Study}

https://www.sheffield.ac.uk/library/digitalpreservation/casestudy

Una sintesi del processo di acquisizione di un sistema di conservazione digitale presso l'Università di Sheffield. (2 pagine). 


\section{Audit e certificazione}

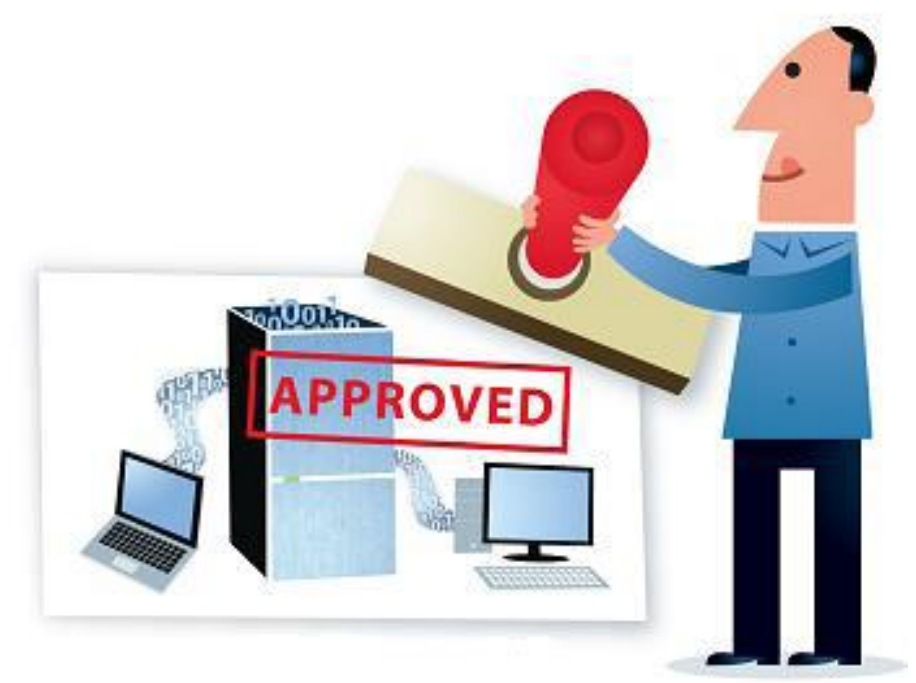

Illustrazione di Jørgen Stamp digitalbevaring.dk CC BY 2.5 Denmark

\section{Introduzione}

Le organizzazioni sono sempre più attente alla verifica delle loro infrastrutture digitali rispetto a un sistema di valutazione, e audit; certificazione e autovalutazione sono temi rilevanti nella conservazione digitale. Vale la pena quindi dedicare uno spazio all'analisi della differenza tra i processi di autovalutazione e l'audit.

Audit e certificazione si riferiscono a un processo formale che generalmente viene portato avanti e garantito da soggetti terzi. Spesso è un procedimento che richiede tempo con delle elevate richieste che dimostrano a un pubblico esterno che ci si sta conformando a standard specifici.

L'autovalutazione è un antesignano, o un'alternativa, per un intero audit e viene effettuata tipicamente dal personale interno all'organizzazione, e i risultati hanno generalmente un valore estremamente importante per l'organizzazione che si sta valutando (rispetto a un pubblico esterno). Le autovalutazioni possono essere utili per identificare delle procedure che richiedono dei miglioramenti, in particolare se un'organizzazione è interessata a portare avanti audit e certificazione completi in un momento successivo.

Si possono riportare molti benefici nell'assicurare che un repository possa essere considerato affidabile. Attualmente, il concetto di un repository digitale affidabile e attendibile è un aspetto ravvisato su vasta scala nella comunità di conservazione digitale. La sezione che segue descrive il lavoro svolto negli ultimi 10-15 anni necessario per arrivare fino a questo punto.

Quadro generale dello sviluppo di controlli e sistemi di certificazioni

I metodi di audit e certificazione per l'implementazione della conservazione digitale sono in fase di sviluppo da almeno un buon decennio all'interno di diverse organizzazioni che in parallelo 
implementano diverse metodologie. In Europa si trovano in coalescenza con l'European Framework for Audit and Certification of Digital Repositories.

II modello di riferimento OAIS (https://www.dpconline.org/handbook/institutionalstrategies/audit-and-certification) (si veda Standard e buone pratiche) ha influito sullo sviluppo di diverse metodologie, a partire dalla pubblicazione dei Trusted digital repositories: Attributes and responsibilities (RLG/OCLC, 2002). È stato poi perfezionato come una pubblicazione bozza 'Una lista di controllo audit per la certificazione di repository digitali affidabili' (RLG-NARA, 2005) prima di essere completata come TRAC (Trustworthy Repositories Audit \& Certification: Criteria and Checklist) ( $\underline{\mathrm{CRL}, 2007)}$.

Sia i Paesi Bassi che la Germania hanno portato avanti un'attività simile. II processo di autovalutazione, Data Seal of Approval sviluppato da DANS (Data Archiving and Networked Services), è stato presentato nel 2008. Nel frattempo, sulla base delle raccomandazioni di un gruppo di lavoro di NESTOR, la German Standards Committee (DIN) ha adottato DIN 31644 Information and documentation - Criteria for trustworthy digital archives.

In coda alla loro pubblicazione dello standard OAIS, e poi all'adozione di OAIS come standard ISO, nel settembre 2011 la Consultative Committee for Space Data Systems ha presentato la raccomandazione su "Audit and certification of trustworthy digital repositories". Successivamente, questo è stato adottato e pubblicato come ISO 163632012 Audit and certification of trustworthy digital repositories (ISO, 2012b).

Attuali modelli di valutazione e il Sistema Europeo per l'Audit e la Certificazione (European Framework for Audit and Certification)

L'apparente proliferazione di standard di repository audit, è stata spesso ritenuta un deterrente per la partecipazione. Di conseguenza, la Commissione Europea ha ospitato una serie di incontri per discutere dell'approccio europeo su vasta scala, e ora si ha a disposizione il Memorandum of Understanding per definire lo European Framework for Audit and Certification of Digital Repositories. Questo protocollo crea in effetti un approccio multilivello alla certificazione permettendo una valutazione a livello base e una revisione tra pari basata sulla CoreTrustSeal (in precedenza, Data Seal of Approval), un'autovalutazione più completa (basata su DIN 31644 o ISO 16363), e un audit esterno su vasta scala basato sulla ISO 16363.

\section{Sigillo di approvazione dei dati}

II Sigillo di approvazione dei dati (Data Seal of Approval DSA, 2008) è un processo di autovalutazione per gli archivi digitali, rivolto in modo specifico a quegli archivi che detengono dati. Nonostante sia richiesto un investimento di tempo per l'applicazione del DSA, è pur sempre meno oneroso dell'ISO 16363 poiché ha solo sedici linee guida sulle quali l'organizzazione viene valutata. Le linee guida sono basate sui seguenti quattro criteri:

- I dati possono essere trovati su Internet;

- I dati sono accessibili (diritti definiti e licenze);

- I dati sono in un formato utilizzabile; 
- I dati sono affidabili;

I dati vengono identificati in modo unico e persistente al fine di potervi fare riferimento, nonostante la DSA sia in apparenza un controllo interno, questa autovalutazione viene revisionata prima che venga assegnato un sigillo, aggiungendo così un livello di autorevolezza al processo. Vengono favorite l'apertura e la trasparenza e alle istituzioni viene chiesto di rendere disponibili le proprie testimonianze in forma gratuita online (fondamentalmente la documentazione, le politiche e le procedure). A differenza dell'audit in linea con lo standard ISO 16363, la revisione non deve controllare l'istituzione per verificare se le politiche e le procedure sono messe in pratica, quindi questo processo si basa molto sulla fiducia.

La DSA si trova nelle fasi finali della revisione di modifiche proposte alle Linee guida DSA come risultato del lavoro con il World Data System attraverso la Research Data Alliance. I dettagli sul quando e sul come la transizione alle nuove linee guida sarà gestita verranno pubblicati a tempo debito, ma nel frattempo l'attuale sigillo è stato adottato per tutto il 2017.

2. DIN 31644 Informazioni e documentazione - Criteri di attendibilità degli archivi digitali La DIN Standards Committee in Germania ha adottato la DIN 31644 Information and documentation - Criteria for trustworthy digital archives basata sulle raccomandazioni del gruppo di lavoro del network di competenza in Germania per la conservazione digitale (NESTOR). Lo standard si concentra sulla necessità di disporre di un repository digitale attendibile strutturato in tre sezioni:

II framework organizzativo, prevede che:

- II repository abbia definito gli obiettivi per la sezione di risorse digitali e accetti la responsabilità di conservarle sul lungo periodo;

- II repository abbia identificato una comunità per la quale saranno garantiti l'accesso e la capacità di interpretare le risorse digitali;

- Siano osservate le regole legali e contrattuali tra i creatori di dati e il repository digitale;

- Siano garantite strutture organizzative sufficienti in termini di personale, risorse finanziarie, pianificazione sul lungo termine e continuità del servizio;

- I processi e le responsabilità siano definiti e documentati.

La gestione dell'oggetto, prevede che:

- L'integrità e l'autenticità delle risorse digitali vengano mantenute;

- Venga messo in atto un piano strategico di attività di conservazione digitale;

- Vengano definiti i pacchetti di informazione per ingest, archiviazione e disseminazione;

- Sia fornita una documentazione adeguata che include gli identificatori permanenti e i diritti strutturali e tecnici e modifiche relative ai metadati;

- Le risorse digitali e i metadati corrispondenti siano messi insieme per una conservazione permanente. 
L'infrastruttura e la sicurezza, prevede che:

- L'infrastruttura IT possa occuparsi delle risorse digitali in modo adeguato e che sia sicura.

DIN 31644 è tedesca, ma è presente una traduzione in inglese offerta da NESTOR sul loro sito web.

Il processo di certificazione ampliato e avviato da NESTOR dura circa tre mesi. La guida su questo processo (NESTOR Certification Working Group, 2013) è disponibile sul loro sito web.

Questo processo di certificazione non dovrebbe essere confuso con un audit completamente esterno - quest'ultimo richiede un riconoscimento formale in linea con la ISO 16363.

\section{ISO 16363 Controllo e certificazione dell'attendibilità degli archivi digitali}

Lo standard ISO 16363 è un framework di audit basato su elementi probatori che usa il termine 'repository' in riferimento all'organizzazione che si occupa della conservazione digitale piuttosto che solo all'infrastruttura tecnica che viene usata per l'archiviazione. I criteri utilizzati nello standard si riferiscono a tutta quanta l'organizzazione e non solo al sistema tecnico in cui il contenuto della collezione viene archiviato. Le metriche vengono suddivise in tre aree:

- Infrastruttura organizzativa: include l'amministrazione, la struttura organizzativa, il personale, le responsabilità procedurali, il quadro strategico, la sostenibilità finanziaria e i contratti, la concessione di licenze e le responsabilità;

- Gestione dell'oggetto digitale: acquisizione e ingest, piano di conservazione, creazione e conservazione dell'Archival Information Packages (AIPs), e gestione delle informazioni e degli accessi;

- Gestione dell'infrastruttura e del rischio per la sicurezza: tra queste, infrastruttura tecnica, gestione del rischio e gestione del rischio per la sicurezza.

La terminologia utilizzata all'interno dello standard ISO 16363 viene esplicitamente allineata a quella dell'OAIS e lo standard si interfaccia direttamente sia con i pacchetti informativi dell'OAIS che con le aree funzionali. Perciò, una comprensione di base dell'OAIS è utile per coloro che cercano di capire lo standard ISO 16363 e offrire una valutazione di confronto.

Oltre 100 metriche vengono distribuite sulle tre aree tanto che effettuare un audit di ISO 16363 o valutazione è un impegno significativo che è simile a molti altri standard ISO applicati all'interno delle organizzazioni. Un numero relativamente piccolo di organizzazioni ha usato lo standard ISO 16363 fin dalla sua pubblicazione. Alcune hanno richiesto la certificazione da parte di revisori contabili esterni, mentre altre hanno avviato delle auto-valutazioni. Houghton (2015) ha fatto presente che sebbene l'autovalutazione non sia un audit è comunque un'attività importante che dovrebbe essere commisurata alle situazioni organizzative.

La ISO 16363 segue le prassi per la certificazione ISO che prevedono che chi avvia l'audit sia certificato. Vi sono altri due standard ISO che vi fanno riferimento: 
- ISO 16919 Requirements for bodies providing audit and certification of candidate trustworthy digital repositories (ISO, 2011) stabilisce quali sono i requisiti per qualsiasi organizzazione che certifica i revisori per ISO 16363;

- ISO 17021 Requirements for bodies providing audit and certification of management systems (ISO, 2012a) fornisce un meccanismo per gli organismi di accreditamento di audit.

Un'agenzia chiamata PTAB (Primary Trustworthy Digital Repository Authorisation Body) offre una formazione per i revisori contabili e per coloro che si preparano per l'audit. Altre agenzie che includono il Center for Research Libraries stanno fornendo audit secondo questi standard.

\section{Altri framework e strumenti per l'autovalutazione}

Un'utile risorsa di livello base è il Levels of Digital Preservation di NDSA (NDSA, 2013), in particolare per quelle istituzioni che sono appena agli inizi e può essere usata per i passaggi iniziali di riferimento. I livelli NDSA sono ampiamente utilizzati nel Manuale (si veda Guida introduttiva, Fixity and checksum, Sicurezza delle informazioni, e Archiviazione). Anche i quadri di valutazione del rischio e gli strumenti possono contribuire alle valutazioni di audit (si veda Gestione del rischio e dei cambiamenti).

\section{Quale opzione di audit o valutazione scegliere?}

II Memorandum of Understanding del 2010, già precedentemente menzionato, identifica in effetti un approccio multilivello della certificazione. L'entità dello sforzo richiesto per ogni livello aumenta, nonostante lo faccia anche il modello dell'output. Scegliere un sistema di valutazione per qualsiasi organizzazione dovrebbe, quindi, prevede i seguenti aspetti:

\begin{tabular}{|l|l|}
\hline \multicolumn{2}{|l|}{ Selezionare un quadro di valutazione } \\
\hline Cosa si vuole ottenere dal proprio audit? & $\begin{array}{l}\text { Quale livello di affidabilità si sta cercando di } \\
\text { raggiungere? Si cerca una certificazione da } \\
\text { un'autorità esterna o basta l'autovalutazione? }\end{array}$ \\
\hline $\begin{array}{l}\text { Quanto sforzo o quanti finanziamenti sono } \\
\text { disponibili per garantire la valutazione? }\end{array}$ & $\begin{array}{l}\text { La ISO 16363 è una grande impresa che prevede } \\
\text { diversi sforzi atti a mettere insieme le prove a } \\
\text { disposizione per avviare l'audit; il CoreTrustSeal } \\
\text { presenta meno metriche e può essere } \\
\text { completato in un periodo molto più breve. La DIN } \\
31644 \text { ha due alternative di valutazione che } \\
\text { richiedono sforzi variabili. }\end{array}$ \\
\hline $\begin{array}{l}\text { Che tipo di contenuto ha la propria } \\
\text { istituzione? }\end{array}$ & $\begin{array}{l}\text { Attualmente, il CoreTrustSeal è stato } \\
\text { principalmente sviluppato per repository che } \\
\text { contengono dati, mentre sia la DIN che la ISO } \\
16363 \text { sono indipendenti dal contenuto dei dati. }\end{array}$ \\
\hline
\end{tabular}


Quale sistema, se previsto, avrà più peso nella propria organizzazione o con i propri stakeholder esterni?
Esiste a livello nazionale una preferenza per un sistema di riferimento o ce n'è uno che viene solitamente usato da organizzazioni simili tra loro che bisognerebbe utilizzare?

La scelta di sistemi di valutazione non dovrebbe essere fatta in forma indipendente e spesso può essere direttamente influenzata dal valore che una valutazione potrebbe avere per altre sezioni dell'organizzazione. II fatto di discutere sulle alternative a livello aziendale e amministrativo può rappresentare un passaggio iniziale utile per far sì che venga selezionata l'opzione corretta e che il supporto venga protetto da altre aree dell'organizzazione sin dal principio.

\section{Come avviare un audit o autovalutazione}

Una volta scelta una metodologia appropriata, un modo chiaro di procedere è quello di sviluppare l'iniziativa come un progetto e creare un piano di progetto.

I suggerimenti sulla pianificazione progettuale che si trovano online sono diversi e dovrebbero essere consultati qualora la propria organizzazione non disponesse di un processo concordato per la gestione del progetto.

Se invece dispone di questo processo, allora è bene acquisirne familiarità e pianificare il proprio progetto seguendo questa metodologia (o assicurare il supporto di un progetto amministrativo locale). Il piano dovrebbe includere almeno le seguenti sezioni:

- Ambito: che contenuto sarà valutato?

- Termini: quando avverrà la valutazione e quando verranno dati i risultati?

- Stakeholder: chi consegnerà la valutazione? chi si deve consultare?

- Amministrazione: quale gruppo gestirà la valutazione e i risultati?

- Comunicazioni: come verranno comunicati agli stakeholder il processo e il risultato?

- Prossimi passaggi: come verranno implementati i risultati?

Se si sta avviando una valutazione ISO 16363 si dovrebbero consultare le raccomandazioni su ISO 16363 Primary Trustworthy Digital Repository Authority Body website. La pagina di preparazione di audit mostra i passaggi che dovrebbero essere seguiti nell'avvio di un audit completo, che possono essere poi adattati per un'autovalutazione. Allo stesso modo, il sito web della CoreTrustSeal offre un sistema di autovalutazione online che guiderà nella valutazione. Sono anche disponibili le versioni PDF o HTML delle linee guida sulla valutazione manuale. 


\section{Risorse}

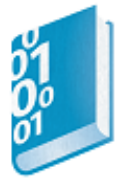

\section{APARSEN Report on Peer Review of Digital Repositories}

http://www.alliancepermanentaccess.org/wp-

content/uploads/sites/7/downloads/2014/06/APARSEN-REP-D33 1B-01-1 1 incURN.pdf

Le lezioni apprese nel processo di certificazione di repository affidabili sono state riprese dal progetto APARSEN in questo report che suggerisce che, sebbene si siano verificati importanti progressi, probabilmente le procedure di audit non sono ancora assestate e permangono alcune questioni sia per i revisori che per i repository. (2012, 50 pagine).

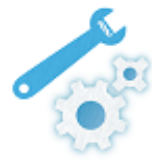

\section{Digital Preservation Management Tools: Principles}

\section{http://dpworkshop.org/workshops/management-tools/principles}

Per le organizzazioni coinvolte per diventare dei Trusted Digital Repositories (TDR ${ }^{22}$ ), un passaggio formativo per lo sviluppo di un processo di conservazione digitale sostenibile e di un programma di curation è quello di adattare e adottare un insieme di principi basati sugli standard come base di partenza. I principi, infatti, forniscono una struttura al proprio programma e il loro utilizzo è un aspetto positivo (e si spera facile) da cui partire.

\section{Digital Preservation Management Tools: Model document http://dpworkshop.org/workshops/management-tools/policy-framework}

Ogni Trusted Digital Repository deve avere un documento strategico di alto livello che dichiari esplicitamente l'ambito, lo scopo, gli obiettivi, i principi operativi, il contesto del piano di conservazione e curation dell'organizzazione. II team DPM che si occupa del workshop ha realizzato questo modello di documento per aiutare le organizzazioni a raggiungere questo obiettivo. Un modello di documento identifica le sezioni raccomandate dal sistema di politiche della conservazione digitale contenenti descrizioni ed esempi.

\section{Digital Preservation Management Tools: Self-assessment and peer review audit} http://dpworkshop.org/workshops/management-tools/self-assessment

Lo strumento di revisione TRAC (Trustworthy Repository Audit and Certification) sviluppato per il workshop DPM.

\footnotetext{
22 Un TDR è un'istituzione la cui finalità principale consiste nel permettere l'accesso sicuro e garantito nel
} lungo periodo alle risorse digitali affidategli, nel corso del tempo, da una comunità. 
The Open Archival Information System (OAIS) Reference Model: Introductory Guide (2nd Edition)

http://dx.doi.org/10.7207/twr14-02

Questo DPC Technology Watch Report del 2014 offre una breve e accessibile guida allo standard OAIS. La terminologia utilizzata all'interno della 16363 è allineata direttamente con quella dell'OAIS. II report aiuterà gli utenti a capire i principi di base di OAIS, utili per comprendere la ISO 16363 e realizzare un'analisi comparativa.

WWW

Digital Preservation Capability Maturity Model (DPCMM)

http://www.securelyrooted.com/dpcmm/

DPCMM è un modello per valutare il livello di maturità strettamente in linea con lo standard OAIS. Permette alle organizzazioni di valutare la loro preparazione nell'ambito della conservazione digitale sulla base di 5 livelli per 15 elementi.

\section{CoreTrustSeal}

https://www.coretrustseal.org/

II Core Trust Seal rappresenta il primo passaggio per la certificazione di repository affidabili. Questa valutazione del repository comprende una lista di 16 punti che può essere utilizzata per un'autovalutazione o revisione.

\section{European Framework for Audit and Certification of Digital Repositories}

http://www.trusteddigitalrepository.eu/

Nel 2010 venne istituita la European Framework for Audit and Certification of Digital Repositories come forma di collaborazione tra la certificazione Data Seal of Approval (DSA), il Repository Audit, il Certification Working Group del CCSDS e il German Standard (DIN 31644) Working Group on Trustworthy Archives Certification, con lo scopo di dare supporto nella creazione di un sistema integrato per operazioni di audit e di certificazione di repository affidabili digitali che sono costituiti da una sequenza di tre livelli, nella prospettiva di migliorare l'affidabilità.

Casi di studio

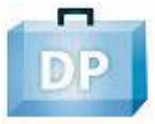

Preserving the H-Net Academic Electronic Mail Lists

http://files.archivists.org/pubs/CampusCaseStudies/Case11Final.pdf

Lisa M. Schmidt, Michigan State University, presenta un'analisi dello stato dell'arte sulla conservazione per le liste di e-mail $\mathrm{H}-\mathrm{Net}$, utilizzando i principi teorici della conservazione digitale dei sistemi di valutazione Trusted Repositories Audit \& Certification: Criteria and 
Checklist (TRAC). Fornisce indicazioni e un sistema di supervisione sull'implementazione dei progressi per rendere $\mathrm{H}$-Net un repository digitale affidabile. Garantire l'autenticità rappresenta un aspetto fondamentale della conservazione. 2009 (15 pagine).

\section{ADS and the Data Seal of Approval - case study for the DCC}

http://www.dcc.ac.uk/resources/case-studies/ads-dsa

Jenny Mitcham e Catherine Hardman, dell'Archaeology Data Service, descrivono l'esperienza di ADS nella richiesta del Data Seal of Approval (DSA), identificando le informazioni pratiche del processo. Inoltre, presentano delle problematiche che ADS ha affrontato durante il processo e i vantaggi potenziali ravvisati dall'auto-certificazione DSA. 2011.

Self-assessment of the Digital Repository at the State and University Library, Denmark a Case Study

https://ipres-conference.org/ipres14/sites/default/files/upload/iPres-Proceedings-final.pdf

In questo articolo di iPres del 2014 gli autori descrivono il processo e i benefici ottenuti nell'esecuzione di un sistema audit basato sull'autovalutazione e su ISO 16363 per il repository digitale della Biblioteca di Stato e Universitaria in Danimarca. (p.272-279 di 385).

\section{TRAC Audit: Lessons}

http://blog.dshr.org/2014/08/trac-audit-lessons.html

La terza di una serie di post sul blog di David Rosenthal riguardo l'audit TRAC del CRL dell'archivio CLOCKSS. I post precedenti avevano anticipato la pubblicazione del report di certificazione e descritto il processo di audit. Questo post presenta le lezioni CLOCKSS, gli altri utenti possono imparare dalle loro esperienze nel processo di audit.

Trustworthiness: Self-assessment of an Institutional Repository against ISO 16363-2012 http://www.dlib.org/dlib/march15/houghton/03houghton.print.html

Nel 2013, la Deakin University Library ha eseguito un'autovalutazione confrontandosi con i criteri dell'ISO 16363. Quest'esperienza ha portato al report che fornisce una valutazione dell'ISO 16363, del processo di analisi, e delle indicazioni per chi ha intenzione di intraprendere una simile avventura.

\section{Managing an ISO 16363 Self-Assessment: A How-To Guide}

http://www.dcc.ac.uk/sites/default/files/documents/IDCC16/18 Managing ISO16363.pdf

Un breve poster presentato durante l'International Digital Curation Conference (IDCC) nel 2016 da Maureen Pennock e Caylin Smith della British Library.

\section{Riferimenti}

CRL, 2007. Trustworthy Repositories Audit \& Certification: Criteria and Checklist. Disponibile al seguente link: http://www.crl.edu/sites/default/files/d6/attachments/pages/trac 0.pdf

DIN, 2012, DIN 31644 Information and documentation - Criteria for Trusted Digital Repositories. Disponibile al seguente link: http://www.nabd.din.de/cmd?level=tpl-art- 
detailansicht\&committeeid=54738855\&artid=147058907\&languageid=de\&bcrumblevel=3\&sub committeeid $=112656173$

Houghton, B., 2015. Trustworthiness: Self-assessment of an institutional repository against ISO 16363-2012. DLib Magazine, 21(3/4). Disponibile al seguente link: http://www.dlib.org/dlib/march15/houghton/03houghton.html

ISO, 2011. ISO 16919:2011 - Space data and information transfer systems - Requirements for bodies providing audit and certification of candidate trustworthy digital repositories. Disponibile al seguente link:

http://www.iso.org/iso/iso catalogue/catalogue tc/catalogue detail.htm?csnumber=57950

ISO, 2012a. ISO 14721:2012 - Space Data and Information Transfer Systems - Open Archival Information System (OAIS) - Reference Model, 2nd edn. Geneva: International Organization for Standardization. Disponibile al seguente link: http://www.iso.org/iso/iso catalogue/catalogue tc/catalogue detail.htm?csnumber=57284

ISO, 2012b. ISO 16363:2012 - Space data and information transfer systems - Audit and certification of trustworthy digital repositories. Geneva: International Organization for Standardization. Disponibile al link: http://www.iso.org/iso/iso_catalogue/catalogue_tc/catalogue_detail.htm?csnumber=56510

NDSA, 2013. The NDSA Levels of Digital Preservation: An Explanation and Uses, version 1 2013. National Digital Stewardship Alliance. Disponibile al seguente link: http://www.digitalpreservation.gov/ndsa/working groups/documents/NDSA Levels Archiving 2013.pdf

nestor Certification Working Group, 2013. Explanatory notes on the nestor Seal for Trustworthy Digital Archives, nestor Materials 17, July 2013. Disponibile al seguente link: http://files.dnb.de/nestor/materialien/nestor mat 17 eng.pdf

RLG/OCLC Working Group on Digital Archive Attributes, 2002. Trusted digital repositories: Attributes and responsibilities, Mountain View, California. Disponibile al seguente link: http://www.oclc.org/content/dam/research/activities/trustedrep/repositories.pdf

RLG-NARA Task Force on Digital Repository Certification, 2005. An audit checklist for the certification of trusted digital repositories, Mountain View. Disponibile al seguente link: https://web.archive.org/web/20051126181100/http://www.rlg.org/en/pdfs/rlgnararepositorieschecklist.pdf 


\section{Conformità giuridica}

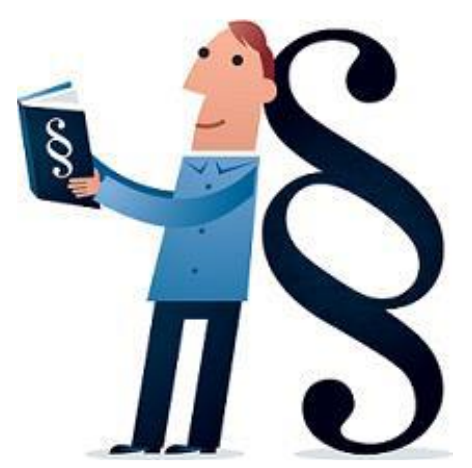

Illustrazione di Jørgen Stamp digitalbevaring.dk CC BY 2.5 Denmark

\section{Introduzione}

In questa sezione vengono date informazioni specificamente relative alle questioni legali che emergono dai vari aspetti dell'archiviazione digitale e dalla conservazione, ma non rappresenta un parere giuridico. Non si cerca, quindi, di offrire una guida sugli aspetti generali giuridici che impattano sulle operazioni delle biblioteche, degli archivi e di altri repository essendo argomenti già trattati in altri lavori di riferimento. Questa sezione è realizzata a partire da una prospettiva inglese e la legislazione in questo ambito cambierà da Paese a Paese. Nonostante siano prettamente riferiti al contesto UK e ad aspetti legali europei, molti degli argomenti sono applicabili in termini generali ad altre giurisdizioni.

Un rapporto consulente-cliente non si base sulle informazioni fornite. Se si necessita di dettagli che riguardano i propri diritti e obblighi, o di un parere legale sull'azione da intraprendere, è bene contattare un consulente legale o un avvocato.

\section{Aspetti giuridici}

'those engaged in digital preservation must work within the law as it stands. This requires both a good general knowledge of what the law is, and a degree of pragmatism in its application to preservation work. Such knowledge enables the archivist to avoid the pitfalls of overcautiousness and undue risk aversion, and to more accurately assess the risks and benefits of taking on the preservation of new iterations of digital work.'(Charlesworth, 2012, p.3)

Diritti di Proprietà intellettuale e conservazione

I Diritti di Proprietà intellettuale (Intellectual property rights (IPR)) sono una sezione del diritto inglese che include al suo interno brevetti, marchi di fabbrica, copyright e diritti associati - come i diritti morali dell'autore (si veda Stakeholder, contratti e condizioni relative alle convenzioni) e i diritti sulle prestazioni. Spesso la conservazione di risorse digitali richiede l'uso di una serie di strategie, e da ciò provengono gli aspetti IPR che probabilmente risultano più complessi e significativi per le risorse digitali piuttosto che per media analogici. Se non vengono affrontati, questi possono pregiudicare o impedire le attività di conservazione. 


\section{Quali sono le differenze riguardo al copyright e agli archivi digitali?}

Tra i diversi IPR, il copyright è di importanza significativa quando si considerano le azioni di conservazione digitale. La legge sul copyright inglese è stata sviluppata avendo come modello l'analogico. Le risorse analogiche tradizionali sono relativamente stabili, e vengono implementati quadri di riferimento normativi e organizzativi già definiti per la conservazione. L'ambito normativo relativo all'attività di conservazione sulle risorse digitali non è ben sviluppato e le pratiche per una corretta conservazione non vengono sempre riconosciute, o consentite, da disposizioni esistenti all'interno della normativa corrente.

Il copyright distingue tra la proprietà della testimonianza fisica del lavoro, come ad esempio un libro o un'opera d'arte, e il diritto individuale di riprodurlo (il diritto a copiare). Per natura, le risorse digitali non si rifanno a questa distinzione e possono creare confusione quando vengono applicate a questo ambito. Nel caso delle risorse digitali, le principali attività di repository, come garantire l'accesso agli utenti e rendere sistematiche le attività di conservazione, spesso implicano la creazione deliberata o accidentale di copie. Senza una chiara specificazione del copyright, licenze o eccezioni legali, queste copie potrebbero costituire delle infrazioni al copyright. Dunque, le risorse digitali definiscono aspetti diversi per i repository che hanno questo tipo di contenuto. Inoltre, a differenza delle risorse fisiche, quelle digitali presuppongono un'attenzione alle dipendenze come hardware e software con le loro specifiche proprietà intellettuali.

Una seconda importante differenza si può rilevare nella durata di vita commerciale e tecnologica relativamente breve delle risorse digitali. La durata dell'IPR nelle risorse digitali va oltre sia il periodo di vita commerciale e, in più o meno tutti i casi, la tecnologia dalla quale dipendono. Tutto ciò crea una situazione triplice: in termini di licenza da procurare per replicare il contenuto, di licenze per software che permettono di accedere al contenuto, e di gestione dei diritti delle risorse digitali "abbandonate", e a tutto ciò si affianca l'urgenza di avviare queste operazioni.

\section{Eccezioni al copyright}

Nonostante il Copyrights, Design and Patent Act (1988) limiti le possibili azioni di conservazione per le risorse digitali, sono state introdotte delle eccezioni per gli archivi, le biblioteche e musei affinché possano gestire le condizioni per la loro conservazione. Dal punto di vista della conservazione, la più importante disposizione (Intellectual Property Office, 2014) è il diritto di produrre un numero qualsiasi di copie richieste al fine di conservare le risorse digitali. Un'altra eccezione importante è quella che permette che una copia digitale (ovvero, una delle copie create sotto eccezione relativa alla conservazione) sia resa disponibile su un terminale dedicato accessibile a utenti esterni. Queste disposizioni si estendono solo a quegli elementi che sono tenuti in forma permanente nelle collezioni. L'eccezione permette alle istituzioni coperte da esenzioni di tenere copie di file in vari formati e perciò conformarsi a ciò che viene considerata una corretta attività di conservazione, sempre in osservazione della legge. Si tenga presente che le disposizioni relative all'eccezione di copyright non annullano i diritti morali dell'autore che devono essere comunque considerati nel momento in cui si avvia l'attività di conservazione. 
Tuttavia, l'esenzione a copiare per la conservazione non prende in considerazione la natura interconnessa delle risorse digitali, e le dipendenze da software di parti terze può ancora costituire un limite alle attività di conservazione. In particolare, questa problematica è frequente nelle strategie di conservazione che si basano in modo decisivo sul mantenimento dell'ambito tecnico di oggetti digitali in questione ad ampio raggio. Ad esempio, l'emulazione come strategia di conservazione prevede l'uso di sistemi operativi originali e software esterni alla collezione permanente del repository (si veda Azione di conservazione). attenzione ai costi aggiuntivi e al tempo impiegato per mantenere dei rapporti con i titolari di diritti di parti terze che derivano dalle necessità non incluse nelle eccezioni.

\section{Opere orfane}

Per le istituzioni che mirano a pubblicare copie digitali di risorse analogiche, l'Orphan Works Licensing Scheme gestito dall'Intellectual Property Office (UK) così come la EU Orphan Works Exception potrebbero avere un impatto determinante sulla digitalizzazione del lavoro e della pianificazione. II Licensing Scheme permette sia la digitalizzazione commerciale (è il caso dei patrimoni istituzionali) che quella non commerciale di qualsiasi tipo di risorsa per cui non è stato possibile tracciare i titolari di diritti della risorsa, seguendo una 'ricerca diligente'. La licenza è un sistema di retribuzione limitato a un periodo di sette anni e per un uso esclusivo nel Regno Unito. D'altra parte, la EU Orphan Works Exception è limitata solo alle opere basate sul testo o audiovisive (e opere artistiche sempre finché ricomprese nelle prime), e a musei, biblioteche, archivi, istituti scolastici e emittenti pubbliche. In questo caso il vantaggio è che le ricerche accurate sono autocertificate e la conservazione della copia di lavoro, creata sotto eccezione relativa alla conservazione, può, ad esempio, essere messa on line per utilizzi non commerciali supportando, così, notevolmente le attività di digitalizzazione.

\section{Accesso e sicurezza}

Una difficoltà in più che si riscontra nell'ambito del copyright si riferisce al fatto che le risorse digitali vengono anche facilmente copiate e ridistribuite. I titolari dei diritti, quindi, sono particolarmente preoccupati circa i controlli degli accessi e le potenziali violazioni del copyright. Le tecnologie Digital Rights Management (DRM), sviluppate per occuparsi di questi aspetti e garantire delle misure di copyright, come ad esempio il software di protezione di copie per i file e gli errori intenzionali fisici ai CD/DVD, possono ostacolare o evitare delle azioni necessarie per la conservazione. Anche le tecnologie DRM sono soggette a obsolescenza. Le questioni legate all'accesso e alla violazione devono essere comprese dalle organizzazioni che conservano risorse digitali nel momento in cui si negoziano contratti di depositi con i titolari dei diritti, e prese in considerazione da entrambe le parti quando si negoziano diritti e procedure per la conservazione. Disporre di procedure chiare in relazione ai depositi può mitigare questioni future di accesso (si veda Diritti di negoziazione).

\section{Archivi web e deposito legale}

Lo status legale degli archivi web e i processi legati al deposito legale elettronico cambiano da Paese a Paese: alcuni governi hanno approvato la normativa relativa al deposito legale ma con accesso limitato solamente alle sale di lettura delle biblioteche. In altri non c'è normativa di 
deposito e le collezioni sono costruite unicamente su basi selettive e di permessi o sono custodite nei 'dark archives' che sono inaccessibili al pubblico. Nel Regno Unito le biblioteche con deposito legale hanno il diritto di raccogliere e garantire l'accesso alle copie di tutti i siti web pubblicati nel dominio inglese. Tuttavia, l'accesso alla collezione è limitato alle sale di lettura della biblioteca (si veda Milligan, 2015). Parallelamente, i Web Archives gestiti dal National Archive (UK) operano su ambiti più piccoli relativi alle pubblicazioni governative e a poteri conferiti dalla legge più chiari che provengono dalla normativa relativa a documenti pubblici (si veda Altre disposizioni normative). La US-based Internet Archive, probabilmente l'archivio web più grande e più utilizzato, non ha autorizzazione normativa esplicita per raccogliere i siti web o pubblicarli. Opera sotto forma di un approccio 'silenzio-assenso' eliminando dalle collezioni ogni sito web che un proprietario richiede venga rimosso. Al contrario, la Library of Congress opera su autorizzazioni, ciò significa che deve esplicitamente richiedere l'approvazione dei titolari di copyright prima di raccogliere o pubblicare i loro contenuti.

\section{Altri obblighi stabiliti dalla legge}

Potrebbero essere applicate anche altre disposizioni normative che, a loro volta, potrebbero condizionare la conservazione delle risorse digitali.

I requisiti riferiti alla normativa relativa ai documenti pubblici e le aspettative riposte nel Freedom of Information Act si applicano ai documenti governativi, anche quelli in forma digitale. I periodi di conservazione normativa e regolamentare si applicano a molti documenti digitali (ad es., per la contabilità e tasse). Nonostante abbiano spesso una durata limitata, si noti che in alcuni settori (ad esempio, industrie farmaceutiche, assistenza sociale e documenti sanitari) le richieste di conservazione di documenti digitali hanno una durata sempre più lunga nel tempo. In questi casi, le strategie di conservazione a lungo termine si applicheranno seguendo i cambiamenti tecnologici che sicuramente incideranno sull'accesso a tali record.

Le informazioni possono essere condizionate dalle norme di protezione dei dati e dalla legislazione pertinente relativa alla privacy che protegge l'informazione detenuta dai singoli. Nel Regno Unito, l'Information Commissioner's Office supervisiona la conformità alla protezione dei dati e alla privacy.

Inoltre, le informazioni possono essere soggette ad accordi di riservatezza. L'attenzione alla privacy e alla confidenzialità potrebbe avere un impatto sulla modalità in cui le risorse digitali possono essere gestite all'interno del repository da parti terze, e rese accessibili per un loro utilizzo. La normativa sulla protezione dei dati incide anche sui movimenti dei dati al di fuori dell'Europa - una considerazione importante per le organizzazioni che investono su un server all'estero.

Le sentenze inglesi sui diritti dei singoli nell'ottenere la rimozione delle loro informazioni personali dai motori di ricerca su Internet in certe circostanze hanno un impatto rilevante sulle attività delle organizzazioni che lavorano con contenuti digitali provenienti dal web (Koops. 2011). L'obbligo di evitare il danneggiamento dei singoli individui nel processo di salvataggio 
dei loro dati su lunghi periodi di tempo si riflette nel principio del diritto all'oblio attraverso l'implementazione dell'Articolo 12 of Direction 95/46/EC nell'ordinamento giuridico di diverse nazioni europee.

\section{Stakeholder, contratti e condizioni di finanziamento}

Alcune risorse digitali sono il risultato di sostanziali investimenti finanziari da parte di finanziamenti pubblici (ad es., consigli della ricerca) e/o editori, e investimenti intellettuali da parte di singoli ricercatori e autori. Ognuno di questi stakeholder potrebbe essere interessato alla conservazione; l'organizzazione che si occupa della conservazione dovrà acquisire delle autorizzazioni dagli stakeholder per proteggere e massimizzare l'investimento economico o il valore intellettuale e culturale dell'opera per le generazioni future. Questi interessi potrebbero concretizzarsi in contratti, licenze e sovvenzioni, o attraverso delle disposizioni normative come i 'diritti morali' per gli autori.

\section{Investimento nelle risorse depositate in repository}

I titolari delle risorse nel corso di molti decenni avranno sicuramente bisogno di investire in risorse per la creazione dei metadati e di una documentazione aggiornata, nonché di nuove versioni delle stesse se si dovrà preservarne l'accesso. In questa nuova forma di investimento, dovranno essere anticipati gli aspetti integrativi nell'IPR e presi in considerazione i reimpieghi futuri di queste risorse. Quando un depositante o titolare di licenza si riserva il diritto di ritirare delle risorse dall'archivio e su queste risorse potrebbero essere previsti nel tempo degli investimenti significativi da parte dell'ente finanziatore, potrebbero essere definiti all'interno degli accordi di deposito e dei canoni di ritiro per la compensazione di qualsiasi investimento (si veda Diritti di negoziazione).

\section{Gestione dei diritti}

Così come descritto nella sezione Aspetti legali, è importante che le questioni legate alle licenze, copyright e ogni altro diritto di proprietà intellettuale sulle risorse digitali che devono essere conservate, vengano chiaramente individuate e le condizioni di accesso concordate con il depositante e/o i titolari dei diritti. Se la titolarità giuridica di questi diritti non è resa chiara 0 risulta eccessivamente frammentata, potrebbe diventare non facile conservare le risorse, così come può risultare difficile l'accesso da parte degli utenti. La gestione dei diritti, quindi, dovrebbe essere trattata come parte dello sviluppo delle collezioni e delle procedure di acquisizione, e dovrebbe essere integrata alle strategie istituzionali di conservazione. II livello di controllo o l'ambito di applicazione della negoziazione da parte delle istituzioni sui diritti varieranno, ma nella maggior parte dei casi le strategie istituzionali in questo ambito saranno di supporto per l'iter operativo. Ciò sarà importante per qualsiasi metadato di conservazione (si veda Metadati $\underline{\text { e documentazione) }}$ e gestione dell'accesso (si veda $\underline{\text { Accesso }}$ ).

\section{Negoziazione dei diritti}

Visto l'aumento del volume delle risorse digitali, della complessità dei diritti, e del numero dei titolari dei diritti nei media digitali, una negoziazione ad hoc tra le agenzie di conservazione e i detentori, e tra gli stessi titolari dei diritti, diventa più complessa e meno efficiente. Questo è 
particolarmente problematico per ogni organizzazione inglese o attività che non è coperta da deroghe al copyright.

Lo sviluppo di modelli di lettera per i diritti di compensazione degli impiegati, accordi, modello di deposito, e licenze modello e clausole che coprano le relative attività di conservazione, aiutano a snellire e semplificare tali negoziazioni. Le istituzioni dovrebbero cercare l'assistenza di un consulente legale per la stesura di una bozza di questi modelli e per fornire una guida per il personale sull'implementazione o modifiche ammissibili nelle negoziazioni con i titolari dei diritti.

Diverse istituzioni hanno realizzato dei modelli che possono essere adottati e adattati a specifiche istituzioni e condizioni. Le procedure di seguito riportate sono una sintesi delle buone pratiche attuali.

\section{Procedure raccomandate}

- Usare un supporto legale per inquadrare la propria politica di gestione dei diritti e per realizzare i documenti.

- Realizzare modelli di lettera per la gestione dei diritti, modelli di accordi di deposito, modelli per licenze e clausole per le attività di conservazione.

- Se si stanno autorizzando delle risorse da parti terze, è bene assicurarsi che queste si siano occupate dell'accesso futuro a risorse a cui si è abbonati e abbiano delle solide prassi per supportare questo processo.

- Preparare confronti motivati e spiegazioni sulle proprie attività di conservazione adatte per gli stakeholder esterni, ad esempio i titolari dei diritti che avranno bisogno di essere convinti di questa necessità e persuasi che i loro interessi saranno salvaguardati. Tenere a mente che per questi ultimi le questioni di conservazione potrebbero essere di poco conto.

- Conservare registri dettagliati delle negoziazioni sui diritti. Creare un programma che identifichi in modo chiaro la lista di risorse depositate e protette da licenza. Questo garantirà che tutto ciò che si ritiene sia stato inviato dal depositante sia stato ricevuto e potrebbe creare le basi della ricezione della comunicazione.

- Conservare le informazioni relative ai diritti e alle autorizzazioni per tutte le proprie risorse digitali. II trattamento delle licenze, dei programmi, e i diritti alla corrispondenza come record istituzionali principali devono essere conservati in ambienti ignifughi e sicuri.

\section{Sintesi degli aspetti legati alle licenze e accordi di deposito}

Di seguito si riporta una breve lista di controllo e sintesi degli aspetti legali che si dovrebbero tenere in considerazione relativamente alle licenze per la conservazione o agli accordi di deposito per le risorse digitali. Le necessità varieranno a seconda delle istituzioni, settori e paesi, e la lista dovrebbe poi essere adattata a richieste individuali. Questa lista non costituisce un parere legale, per i casi specifici personali si deve ricercare un consulente legale.

\section{IPR e la conservazione digitale}

Una clausola dovrebbe essere redatta al fine di coprire i seguenti aspetti: 
- Permessi necessari per il contenuto.

- Permessi necessari per il software associato.

- Permessi necessari per la copia ai fini della conservazione. (Sezione che è applicabile a risorse o organizzazioni non protette da eccezioni al copyright)

- Permessi necessari per migrare in futuro il contenuto verso nuovi formati ai fini della conservazione. (Sezione che è applicabile a risorse o organizzazioni non protette da eccezioni al copyright)

- Permessi necessari per l'emulazione ai fini della conservazione.

- Permessi rispetto ai meccanismi di protezione del copyright.

\section{Accesso}

- Permessi e condizioni rispetto all'accesso alle risorse.

\section{Aspetti normativi e contrattuali}

- Permessi normativi e obblighi legali relativi al deposito rispetto alle risorse digitali.

- Concessioni e obblighi contrattuali rispetto alle risorse digitali.

- Condizioni, diritti e interessi appropriati degli autori, editori e altri finanziatori.

- Informazioni confidenziali e protezione della confidenzialità degli individui e delle istituzioni.

- Protezione dell'integrità e reputazione dei creatori dei dati o di altri stakeholder.

Investimenti da parte di un'agenzia di conservazione

- IPS in ogni valore aggiunto dall'agenzia di conservazione

- Clausole di recesso (e pagamenti associati).

\section{Risorse}

La normativa britannica viene periodicamente aggiornata. Per essere certi che si stia accedendo agli ultimi aggiornamenti, si prega di visitare direttamente l'indirizzo http://www.legislation.gov.uk/

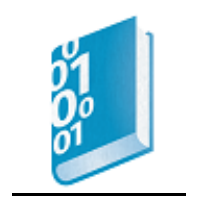

\section{Exceptions to Copyright: Libraries, Archives and Museums}

https://www.gov.uk/government/uploads/system/uploads/attachment data/file/375956/Librarie

\section{$\underline{s \text { Archives and Museums.pdf }}$}

Questo opuscolo di orientamento pubblicato dall'Intellectual Property Office nel 2014 definisce le eccezioni che possono essere applicate alle biblioteche, agli archivi e ai musei. È importante per chiunque lavori all'interno di archivi o con archivi, musei o biblioteche nel Regno Unito, o per i titolari di copyright il cui contenuto è gestito da tali istituti. Si interessa di due importanti cambiamenti nel sistema normativo britannico che riguardano le biblioteche, gli archivi e i musei. 
II primo si riferisce alle copie delle opere che si realizzano nella prospettiva di una loro conservazione per generazioni future. II secondo permette una libertà maggiore nel copiare le opere per chi porta avanti ricerche non commerciali e studi privati.

\section{Intellectual Property Rights for Digital Preservation \\ http://dx.doi.org/10.7207/twr12-02}

Questo report sull'osservazione dello sviluppo tecnologico della DPC venne reso pubblico nel 2012 da parte di Andrew Charlesworth. II documento non copre la normativa recente (come ad esempio, The Legal Deposit Libraries (Non-Print Works) Regulations 2013 e 2014 Copyright Exceptions for Libraries, Archives and Museums), tuttavia rappresenta un importante lavoro introduttivo. Come utenti finali principali vi sono i detentori, gli archivisti e i ricercatori/riutilizzatori delle opere digitali. II diritto di proprietà intellettuale, rappresentato in massima parte dal copyright e dai suoi rispettivi diritti, finora ha costituito uno dei principali effetti legali sulla conservazione digitale, spesso non trattabile. È fondamentale per chi ha a che fare con la conservazione digitale per essere in grado di identificare e implementare strategie pratiche e pragmatiche nella gestione dei rischi legali relativi ai diritti di proprietà intellettuale per il raggiungimento di obiettivi legati alla conservazione e all'accesso. (54 pagine).

\section{Aligning National Approaches to Digital Preservation \\ https://educopia.org/wp-}

\section{content/uploads/2018/06/Aligning National Approaches to Digital Preservation.pdf}

Questi Atti del 2012 comprendono due articoli sui seguenti aspetti legali: Legal Alignment di Adrienne Muir, Dwayne Buttler, e Wilma Mossink (pp. 43-74); e Legal Deposit and Web Archiving di Adrienne Muir (pp. 75-88). Il primo articolo si concentra sugli aspetti principali del deposito legale, sulle eccezioni al copyright per la conservazione e l'accesso, sul il lavoro con diversi partner e transfrontaliero e sulla gestione dei diritti; il secondo descrive le sfide legate all'adeguamento del deposito legale da un meccanismo pensato per la pubblicazione su stampa a uno per pubblicazioni destinate all'ambiente digitale. Vengono identificati gli approcci nazionali relativi agli aspetti centrali alla struttura di riferimento di deposito legale e alle problematiche legali che emergono dagli approcci non regolamentari per la collezione di pubblicazioni digitali nella prospettiva di una conservazione a lungo termine. (342 pagine).

\section{Cloud Storage Guidance Appendix Table 3 - Legal Issues}

http://www.nationalarchives.gov.uk/documents/CloudStorage-Guidance March-2015.pdf

La Tabella 3, presente nella sezione 7 come appendice alla TNA Cloud Storage Guidance, pubblicata nel 2015, elenca nel dettaglio una serie di aspetti legali per ognuna delle tre categorie principali:

- Qualsiasi requisito legale riferito alla gestione, conservazione e accesso posto sugli archivi e le loro società madre, da parte dei loro donatori e finanziatori mediante contratti e accordi o legislazioni governative (ad es., accessibilità, disponibilità, sicurezza delle informazioni, conservazione, audit e conformità, Public Records Act, etc.); 
- Gli obblighi legali relativi ai diritti di parti terze nei/sui dati che devono essere archiviati (ad es. copyright, protezione dei dati); e

- Gli elementi legali del rapporto esistente tra un archivio e un fornitore o fornitori di servizi cloud (ad es. termini di servizio dei contratti e accordi sul livello di servizio).

\section{Archives and Copyright: Risk and Reform, CREATe Working Paper No.3}

http://www.create.ac.uk/wp-content/uploads/2013/04/CREATe-Working-Paper-No-3-v1-1.pdf Le pagine 6-18 di questo paper di 58 pagine del 2013 di R. Deazley and V. Stobo riguardano il Copyright e il settore archivistico nell'ambito britannico.

\section{E-ARK Legal Issues Report: European Cultural Preservation in a Changing Legislative Landscape \\ http://www.eark-project.com/resources/project-deliverables/33-d22-legal-issues-report- european-cultural-preservation-in-a-changing-legislative-landscape/file}

Questo report del 2017 di David Anderson della Brighton University e del progetto E-ARK si interessa della legislazione Europea e del suo impatto sulla Conservazione Digitale. La parte principale del report riporta i requisiti previsti nella Direttiva 95/46/EC che è stata implementata dagli Stati Membri con una serie di strumenti legislativi a partire dall'attuazione della Direttiva nel 1995. Questi ultimi sono stati impostati sulla stregua delle proposte del General Data Protection Regulation (GDPR), oggetto di discussione tra la Commissione, il Consiglio dei Ministri e Parlamento Europeo. Non essendo stato raggiunto un accordo sulla versione finale del testo al momento della stesura, alcune delle conclusioni interne a questo report risultano, per forza di cose, provvisorie. (130 pagine).

\section{Legalities Life Cycle Management}

https://www.timbusproject.net/portal/domain-tools/334-lehalities-lifecycle-management-tools/ Uno strumento sviluppato dal progetto TIMBUS che si occupa della conservazione digitale dei processi aziendali. Le aree trattate sono IPR, contrattualistica IT, Protezione dati e altri requisiti regolamentari.

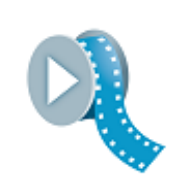

\section{Mass Digitization of Cultural Heritage: Can Copyright Obstacles Be Overcome? http://livestream.com/unc-sils/iPres-Pamela-Samuelson/videos}

Un Keynote iPRES del 2015 di Pamela Samuelson, professoressa di Diritto e Informazione presso la University of California, Berkeley. Samuelson è autrice di una serie di pubblicazioni su IPR e Cyberlaw. In questa presentazione considera il ruolo dell'"utilizzo corretto" quando si 
tratta di affrontare delle sfide poste dal Copyright. Parla da una prospettiva giuridica americana, ma molte delle sue considerazioni si possono applicare al contesto britannico. (2015) 56 minuti.

\section{Riferimenti}

Charlesworth, A.J., 2012. Intellectual Property Rights for Digital Preservation, DPC Technology Watch Report 12-02. Disponibile al seguente link: http://dx.doi.org/10.7207/twr12-02

Intellectual Property Office, 2014. Exceptions to Copyright: Libraries, Archives and Museums. Disponibile al seguente link:

https://www.gov.uk/government/uploads/system/uploads/attachment data/file/375956/Librarie $\underline{\mathrm{s} \text { Archives and Museums.pdf }}$

Koops, B., 2011. Forgetting Footprints, Shunning Shadows. A Critical Analysis of the "Right To Be Forgotten" In Big Data Practice. SCRIPTed, 8:3, 229-256. Disponibile al seguente link: http://script-ed.org/wp-content/uploads/2011/12/koops.pdf

Milligan, I., 2015. Web Archive Legal Deposit: A Double-Edged Sword. Disponibile al seguente link: http://ianmilligan.ca/2015/07/14/web-archive-legal-deposit-a-double-edged-sword/ 


\section{Gestione del rischio e dei cambiamenti}

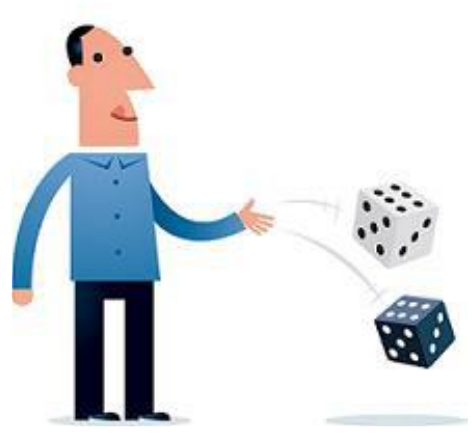

Illustrazione di Jørgen Stamp digitalbevaring.dk CC BY 2.5 Denmark

\section{Introduzione}

La conservazione digitale non si occupa semplicemente di rischi, ma crea anche delle opportunità e, proteggendo le risorse digitali, fa in modo che da queste ne derivi un valore nuovo o aggiunto. È facile essere circondati da rischi, perciò conviene essere chiari, sin dal principio, riguardo a quali opportunità vadano protette o create. Ci sono diversi fattori che mettono le proprie risorse digitali a rischio, tra cui i cambiamenti nella propria organizzazione o nelle tecnologie. Se questi rischi non vengono gestiti, avranno un impatto significativo sulla capacità di portare avanti le attività di conservazione digitale, sulle più ampie funzioni aziendali, o sulla conformità alla normativa.

Per gestire la conservazione digitale, si deve capire quali sono gli aspetti specifici e i rischi della propria organizzazione. Lo si può fare eseguendo un'analisi valutativa dei rischi e delle opportunità. Questa valutazione evidenzierà i rischi specifici riguardo alla continuità delle proprie risorse digitali e le opportunità che possono essere create a partire dalla mitigazione di questi rischi.

\section{Gestione del rischio}

È comprovato che nel momento in cui ci si occupa di risorse digitali i rischi siano sottili e svariati. Questi rischi includono (ma non sono limitati a):

- Fusione, chiusura o trasferimento di funzioni tra organizzazioni.

- Cambiamenti nella direzione strategica o nell'area finanziaria, e nelle funzioni supportate dalle organizzazioni.

- Variazione dei ruoli tra i singoli leader o esperti.

- Analisi dei dati di rilevamento delle risorse come conseguenza della difficoltà riscontrata nel recupero dei dati.

- Perdita del copyright o di altre informazioni legali causando incertezza sui diritti e sugli obblighi.

- Esternalizzazioni senza prendere in considerazione future necessità di conservazione.

- Obsolescenza del formato dei file, e ciò significa che processare i dati risulta o costoso o impossibile. 
- Obsolescenza dei media che rende il recupero dei dati costoso o impossibile.

- Degrado dei media, con ciò si intende che i dati sono danneggiati o modificati.

- Perdita delle informazioni contestuali che provocano una perdita di significato.

- Perdita della provenienza delle informazioni o fixity riguardo ai documenti, implicando una perdita di autenticità.

- Interruzione del controllo della versione che rende difficile l'identificazione delle istanze autoritative di un documento.

- Errore umano che porta a una cancellazione involontaria.

- Il livello di utilizzo. Un dark archive è più a rischio di un altro molto utilizzato. Se alle risorse digitali si accede non molto di frequente, l'impatto di fallimento è meno visibile in forma immediata.

- Catastrofi naturali che hanno effetti sugli edifici o sulle infrastrutture.

La perdita dei dati potrebbe portare diverse conseguenze nel mondo reale a seconda del contesto. Ad esempio, nel contesto di un processo, l'autenticità di un documento potrebbe rappresentare un importante aspetto legale; mentre per dati della ricerca altamente strutturati, la catena di custodia potrebbe essere meno importante rispetto all'accesso al contesto informativo che permette la riproducibilità di un esperimento. In molti contesti potrebbe essere tecnicamente possibile recuperare le collezioni digitali, ma laddove un'organizzazione non abbia semplicemente gli strumenti o le capacità necessarie per ripristinare un dataset, ne risulta una forma di obsolescenza tecnica e la perdita dei dati. È probabile che questo accada nel momento in cui aumentano la quantità e la complessità delle collezioni digitali.

I rischi legati al contenuto digitale solitamente sono un aspetto da tenere in conto a causa delle conseguenze che questi ultimi hanno nella realtà. Anche in questo caso il contesto gioca un ruolo importante, ma possono capitare una serie di cose tra cui:

- La perdita della reputazione.

- Risorse inadeguate per un compito difficile.

- Incapacità di aiutare gli utenti nelle loro attività.

- Insuccesso nello svolgimento di funzioni legali o regolamentari.

- Incapacità nello sfruttare e riutilizzare i dati.

- Perdita della memoria identitaria e aziendale.

Tipicamente, si tende a dare priorità ai rischi calcolando il 'punteggio di rischio' basato su probabilità, impatto e imminenza: un rischio imminente con una forte probabilità e un impatto negativo esteso necessita di interventi immediati.

A seconda della natura del rischio si possono prendere iniziative per ridurre la probabilità di un rischio emergente, ridurre l'impatto se dovesse sussistere il rischio, o prendere tempo per mitigare i passaggi che devono essere implementati.

La valutazione del rischio rappresenta un processo costante che può essere sviluppato ed esteso nel tempo. Può aiutare a riunire differenti stakeholder e, visto che la gestione del rischio 
è un aspetto trattato dai dirigenti, anche a facilitare una forma di investimento. Anche una valutazione del rischio minima evidenzierà quelle priorità che servono a chiunque si approcci alla conservazione digitale.

Infine, si noti anche che la conservazione digitale è specifica nel lungo periodo e la maggior parte delle metodologie riferite al rischio è generalmente incentrata sul breve periodo. Per la conservazione digitale si deve essere consapevoli che sul lungo periodo sarà più probabile che capitino eventi improbabili e che si debba prestare particolare attenzione a quelli con conseguenze significative.

\section{Piano di continuità aziendale}

\section{Motivazione}

'Interested parties and stakeholder require that organizations proactively prepare for potential incidents and disruptions in order to avoid suspension of critical operations and services, or if operations and services are disrupted, that they resume operations and services as rapidly as required by those who depend on them.' (ISO/PAS 22399:2007).

II piano e l'attività di continuità aziendale sono ben consolidati nelle professioni IT, tuttavia questo Manuale non vi dedica uno spazio particolarmente dettagliato. Ciononostante, rappresentano delle componenti importanti per assicurare un minimo di conservazione e per dare un contributo significativo alla conservazione digitale. II progetto TIMBUS mette a disposizione diversi webinar sul tema della continuità aziendale e sulla conservazione digitale (si veda $\underline{\text { Risorse)}}$.

Lo sviluppo e l'uso del piano di continuità aziendale, basato su solidi principi, approvato dai dirigenti e attivato da personale formato, ridurranno decisamente la probabilità e la gravità dell'impatto di disastri e incidenti.

Tra i modelli vi è il piano realizzato dalla Data Archive e descritto nella Nota della DPC sulla continuità aziendale. Le organizzazioni potrebbero anche voler considerare l'uso di servizi cloud (si veda Servizi Cloud) come parte della loro pianificazione.

\section{Requisiti}

- Sviluppare un piano di continuità aziendale.

- Assicurarsi che tutto il personale più importante venga formato nelle procedure di continuità aziendale.

- Archivio delle copie sugli standard del settore o altri approvati dai media attuali.

- Archivio delle copie all'interno e all'esterno. Le copie remote dovrebbero essere archiviate a distanza sicura dalle copie in loco al fine di garantire che queste non vengano danneggiate per motivi naturali o per mano umana con conseguenze sulle copie interne.

- Considerare i dati e le competenze come beni e compilarne dei registri.

- Fare in modo che i ruoli e le responsabilità siano identificati e vengano mantenuti. 


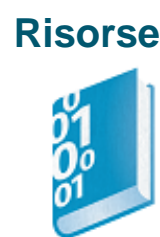

ISO/PAS 22399:2007 Societal security - Guideline for incident preparedness and operational continuity management

http://www.iso.org/iso/catalogue detail?csnumber=50295

Questo standard offre una guida generale ad ogni organizzazione per la definizione dei propri criteri di prestazione in riferimento alla preparazione nella gestione degli incidenti e continuità operativa, e alla progettazione di un adeguato sistema di gestione.

ISO/IEC 27001:2013 Information technology -- Security techniques -- Information security management systems -- Requirements

http://www.iso.org/iso/catalogue detail?csnumber=54534

Questo standard descrive i requisiti per la definizione, implementazione, mantenimento e miglioramento costante di un sistema di gestione della sicurezza delle informazioni. I requisiti sono generici e hanno lo scopo di essere applicati a tutte le organizzazioni.

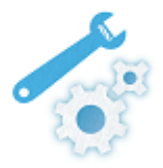

\section{Disaster Preparedness for Digital Content \\ http://dpworkshop.org/workshops/management-tools/disaster-preparedness}

II sito web del workshop del Digital Preservation Management rimanda a un insieme di 4 documenti (politiche sui piani d'emergenza, piani di comunicazione, piani di formazione, ruoli e responsabilità). Insieme, questi ultimi forniscono una documentazione completa e vengono aggiornati per rispecchiare le politiche attuali sulla preparazione alle situazioni di emergenza.

\section{National Archives Risk Assessment Tools}

http://www.nationalarchives.gov.uk/information-management/manage-information/policy-

process/digital-continuity/risk-assessment/

II National Archives offre due strumenti di autovalutazione in formato excel che si ricollegano alla sua guida sulla continuità digitale e sistema di riferimento delle soluzioni e dei servizi. Lo strumento di autovalutazione $(0.4 \mathrm{Mb})$ suddivide la valutazione del rischio in tre parti: capire la continuità digitale e i ruoli e le responsabilità; requisiti riferiti alle informazioni e dipendenze tecniche; gestione.

Lo strumento di valutazione del rischio sui contenuti delle informazioni $(0.26 \mathrm{Mb})$ aiuta nell'identificazione dei rischi per la continuità di ogni asset specifico di informazioni digitali e rileva dove questa continuità sia stata persa. Offre, inoltre, delle raccomandazioni sul mantenimento e ripristino della continuità come supporto allo sviluppo di un piano d'azione della continuità digitale. 


\section{DRAMBORA (Digital Repository Audit Method Based on Risk Assessment) Toolkit}

http://www.repositoryaudit.eu

Un toolkit disponibile online per il digital repository audit. Guida gli utenti al processo di audit, a partire dalla definizione dello scopo e dell'ambito dell'audit fino all'identificazione e gestione dei rischi per il repository. DRAMBORA fornisce una lista di più di 80 esempi di rischi potenziali per i repository digitali, considerati in relazione a delle possibili conseguenze.

\section{SPOT}

http://www.dlib.org/dlib/september12/vermaaten/09vermaaten.html

SPOT (Simple Property-Oriented Threat) è un modello semplice di valutazione del rischio, incentrato sulla protezione alle minacce di sei proprietà degli oggetti digitali, fondamentali per la loro conservazione: disponibilità, identità, persistenza, rappresentazione, comprensibilità, e autenticità. II modello si riferisce alle minacce in termini di potenziali impatti su queste proprietà e presenta una serie di esempi per ognuna. L'articolo descrive il modello e include anche una comparazione di altri modelli di minacce per la conservazione digitale.

WWW

Managing digital continuity guidance from The National Archives

http://www.nationalarchives.gov.uk/information-management/manage-information/policyprocess/digital-continuity/

Comprende un'utile valutazione del rischio con diverse correlazioni alle strategie di gestione del rischio per la pianificazione della continuità aziendale.

Assess and manage risks to digital continuity

http://www.nationalarchives.gov.uk/information-management/manage-information/policy-

process/digital-continuity/step-by-step-guidance/step-3/

II National Archives ha realizzato uno strumento di autovalutazione per il settore di pubblico più ampio che si ricollega alla sua guida sulla continuità digitale e sistema di riferimento per le soluzioni e servizi.

Assess risks to digital continuity factsheet

http://www.nationalarchives.gov.uk/documents/information-management/assess-dc-risks$\underline{\text { factsheet.pdf }}$

(2 pagine)

Risk assessment handbook

http://www.nationalarchives.gov.uk/documents/information-management/Risk-Assessment-

Handbook.pdf

(35 pagine) 


\section{The Atlas of Digital Damages}

\section{https://www.flickr.com/groups/2121762@N23/}

Uno spazio in cui raccogliere esempi visivi delle sfide relative alla conservazione digitale, alle visualizzazioni non riuscite, ai danni riferiti alla codifica, ai dati corrotti, e alle evidenze visive che documentano i \#FAILs di ogni banda. È possibile aggiungere semplicemente un'immagine, raccontarne la storia che vi è dietro, o condividere il file originale (o insieme di file), così che gli sviluppatori dello strumento possano capire il danno digitale e fare una prova del loro codice programma.

TIMBUS project: Business Continuity Management 1 - Intro, Life Cycle, Planning, Scope https://www.youtube.com/watch?v=25EhtuE3XkE

Uno dei quattro webinar di Business Continuity Management e Digital Preservation of Processes all'interno del progetto Timbus finanziato dall'UE. Probabilmente è l'introduzione più accessibile agli utenti principianti (pubblicata nel 2013. 13 minuti).

\section{Casi di studio}

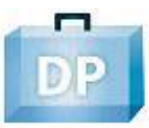

DPC case note: Business continuity procedures - UK Data Archive, University of Essex https://www.dpconline.org/blog/1094-business-continuity-procedures-uk-data-archiveuniversity-of-essex

II Data Archive è il centro dati nazionale britannico per le Scienze Sociali finanziato dall'Economic and Social Research Council (ESRC). L'Archivio detiene la certificazione ISO 27001, lo standard internazionale per la sicurezza delle informazioni, che prevede una continuità della sicurezza delle informazioni da integrare nei sistemi di gestione della continuità aziendale di un'organizzazione. II sistema di archiviazione digitale del Data Archive, per finalità legate alla sicurezza, è basato su archivi e accessi separati e distribuiti. La continuità aziendale presso il Data Archive è basata sulla resilienza data dalla creazione di copie multiple dei dati e da procedure specifiche di recupero, insieme alla prevenzione in forma anticipata di guasti. Ogni file di ciascun dataset ha almeno tre copie. L'Archivio crea anche una copia d'archivio di sola lettura per ogni studio e ogni aggiornamento che viene reso disponibile dal sistema.

\section{Riferimenti}

ISO, 2007. ISO/PAS 22399:2007. Societal security - Guideline for incident preparedness and operational continuity management. Disponibile al seguente link: http://www.iso.org/iso/catalogue detail?csnumber $=50295$ 


\section{Formazione del personale e sviluppo}

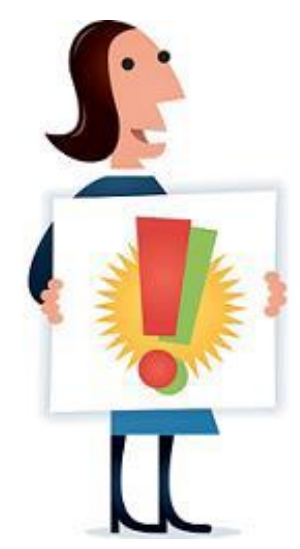

Illustrazione di Jørgen Stamp digitalbevaring.dk CC BY 2.5 Denmark

\section{Introduzione}

Un personale efficiente e competente può rappresentare uno dei maggiori valori di un'organizzazione. Tuttavia, la diligenza e l'attenzione non sempre mirano a offrire una formazione e una crescita adeguate e a esortare alla loro diffusione. Inoltre, lo sviluppo e il mantenimento di un programma di conservazione digitale possono sembrare scoraggianti sotto diversi punti di vista, e questo, in particolare, spesso avviene a causa di un divario esistente nelle competenze del personale. Ciò potrebbe essere dovuto all'ambiente di lavoro che è caratterizzato da:

- Cambiamenti rapidi e costanti.

- Confini offuscati all'interno e tra istituzioni.

- Incertezza in termini di capacità di previsione, in modo sicuro, degli andamenti e delle esigenze future.

- Ruoli e responsabilità non chiari e/o in cambiamento.

- Enfasi accentuata sulla collaborazione e lavoro di squadra.

- Enfasi accentuata sulla responsabilità.

La formazione del personale attentamente progettata e le attività di sviluppo continuo professionale (CPD) possono contribuire in modo decisivo a realizzare efficacemente la transizione dal modello tradizionale delle biblioteche e degli archivi al digitale o al modello ibrido. Un tipo di formazione e un tipo di crescita adeguati possono rappresentare un fattore importante per favorire lo sviluppo di un senso di fiducia e di competenza tra i membri del personale, nonché per minimizzare il livello di ansia relativo alla natura variabile del lavoro nelle istituzioni che si occupano di conservazione. Un approccio attento alla formazione e allo sviluppo (opposto a quello basato sul 'mandare le persone ai corsi' solamente) potrebbe fare molta differenza:

- Aiutare il personale a utilizzare in modo efficiente le tecnologie e a migliorare la qualità complessiva del servizio.

- Aumentare il livello individuale di soddisfacimento lavorativo e l'impegno, portando a una fidelizzazione del personale migliore. 
- Migliorare le prospettive strategiche per l'organizzazione vista come un insieme.

Le organizzazioni dovrebbero attuare un approccio strategico per la formazione e lo sviluppo, considerando in modo ponderato le capacità che sono necessarie così come i ruoli e le responsabilità nuove ed emergenti. Questo punto dovrebbe essere chiarito esplicitamente in tutte le politiche pertinenti alla conservazione digitale, alla strategia e pianificazione e al budget per le attività di sostegno e sviluppo delle competenze che dovrebbero essere parte integrante della pianificazione per il lavoro di conservazione digitale.

\section{Un'ampia gamma di competenze}

Una conservazione digitale di successo prevede l'impiego di una vasta gamma di competenze, da quelle specifiche riferite ad aree come la conoscenza degli standard sui metadati e sui sistemi di audit, fino alle competenze più generali come la pianificazione progettuale e la gestione del rischio. Perciò, far sì che tutti i membri del personale siano in possesso di competenze adeguate in riferimento al ruolo che assumono nel processo, quelle specifiche della conservazione digitale rappresentano solo uno degli aspetti della formazione prevista per preparare questi ultimi alla massimizzazione del potenziale della tecnologia digitale. È molto poco probabile che una persona presenterà tutte le competenze richieste per portare avanti l'insieme completo di attività di conservazione digitale, per cui la collaborazione rimarrà un fattore importante per la riuscita. Una formazione professionale può sviluppare capacità e competenze personali, ma può altresì potenziare il processo di acquisizione di nuove previste per un progetto collaborativo di successo.

Una serie di diverse iniziative ha cercato di chiarire quali competenze e capacità siano richieste per l'attività di conservazione digitale e quali i potenziali ruoli previsti per il personale a seconda dei diversi gradi di anzianità:

\section{DPOE}

La Library of Congress's Digital Preservation Outreach and Education programme (DPOE) ha definito tre livelli relativi ai ruoli del personale (o fasi della carriera) all'interno dei suoi modelli di formazione per la conservazione digitale:

- Esecutivi - ruoli nella gestione istituzionale dirigenziale.

- Dirigenziali - ruoli che gestiscono i programmi e i servizi di conservazione digitale.

- Pratici - professionisti che lavorano attivamente con le risorse digitali e con le soluzioni di conservazione.

\section{DigCurV}

II progetto DigCurV ha adattato i tre livelli del DPOE all'attività relativa all'individuazione delle competenze principali necessarie per la conservazione digitale. II progetto DigCurV ha esaminato una serie di questioni riferite alla digital curation e alla formazione nell'ambito della conservazione, alle competenze e crescita, creando una molteplicità di risorse utili tra cui un database di opportunità di formazioni disponibili e una struttura curriculare. Descrivendo le competenze necessarie per ognuno dei tre livelli nel modello DPOE, attraverso un insieme di 
obiettivi, la struttura curriculare del DigCurv rappresenta una risorsa molto utile per l'identificazione di un quadro completo riferito alle capacità e competenze necessarie per la conservazione digitale. In particolare, il contesto curriculare DigCurV può aiutare gli utenti a descrivere e comparare i corsi, sviluppare nuove risorse di formazione e mappare le competenze e la consapevolezza di una persona o di un team per l'identificazione di qualsiasi divario esistente nelle competenze.

Ogni obiettivo viene suddiviso in quattro sezioni che comprendono:

- Consapevolezza e Proprietà intellettive

- Qualità personali

- Condotta professionale

- Gestione e Garanzia di qualità

Ognuna di queste poi contiene altre sotto sezioni che riportano dei bilanci generali sulle competenze individuali. Questi bilanci sono predisposti per essere generici al fine di divenire applicabili su vasta scala, nonostante siano disponibili sul sito web del DigCurV esempi di standard specifici o di strumenti riferiti alle competenze.

\section{DigCCurr}

II progetto DigCCurr (Preserving Access to Our Digital Future: Building an International Digital Curation Curriculum) ha generato una matrice a sei dimensioni per l'identificazione e organizzazione delle risorse da trattare per il contesto di conservazione digitale. Questa matrice - Matrix of Digital Curation Knowledge and Competencies - rappresenta un approccio alternativo che potrebbe essere particolarmente utile per le organizzazioni più piccole.

\section{Ruoli e Responsabilità per la Formazione e lo Sviluppo}

I ruoli e le responsabilità devono essere definiti in modo chiaro. II successo della formazione e dei programmi di sviluppo sarà condizionato dal livello entro cui i diversi ruoli e responsabilità si inseriscono. Diviene fondamentale che ognuno degli stakeholder coinvolto nel processo sia soddisfatto pienamente delle varie responsabilità che potrebbero essergli richieste per assicurare una creazione e uno sviluppo efficace, nonché una formazione articolata e un programma di crescita. 
Ruoli e responsabilità degli stakeholder

Ruoli e responsabilità dell'Istituzione:

- Sviluppare una strategia informativa che integri la formazione IT con la missione complessiva dell'istituzione.

- Identificare, consultando il personale di riferimento, un audit di competenze per determinare quali specifiche competenze sono necessarie per raggiungere gli obiettivi organizzativi, inclusa l'esamina delle prospettive per nuove ed emergenti competenze, attività e responsabilità.

- Stabilire un equilibrio tra un'implementazione di competenze specifiche e uno sviluppo efficace delle abilità.

- Garantire delle risorse adatte per la formazione e la crescita.

- Assicurarsi che il personale abbia accesso a strumenti adeguati.

- Assicurare l'accesso a una formazione ed esperienza pratica.

- Considerare strategie come il distaccamento nel breve periodo presso un ente che potrebbe avere più esperienza in un ambito specifico.

- Coinvolgere il personale nella progettazione di programmi di formazione e crescita.

- Agevolare le comunicazioni efficaci interdisciplinari.

- Prendere in considerazione in modo esteso ciò che costituisce la formazione e la crescita (ovvero, la combinazione di corsi formali, sia generici che personalizzati, la formazione informale all'interno dell'organizzazione, il trasferimento di competenze all'interno dell'organizzazione, il networking, etc.).

Ruoli e responsabilità delle Associazioni Professionali:

- Capacità di risposta alla formazione attuale e alle necessità di crescita.

- Abilità a lavorare con le istituzioni per sviluppare pacchetti di formazione al fine di soddisfare le loro richieste.

\section{Ruoli e Responsabilità dei singoli}

- Saper tollerare frequenti cambiamenti.

- Capacità di essere flessibili.

- Capacità di lavorare in team.

- Capacità nel comunicare (e ascoltare) in modo efficace tra i gruppi del personale e all'interno dell'organizzazione dall'alto e dal basso.

- Capacità di seguire in modo attivo la crescita professionale personale attraverso una serie di meccanismi.

- Saper condividere capacità e competenze.

- Capacità di acquisire nuove competenze.

- Capacità di applicare nuove competenze.

\section{Avvio della valutazione delle competenze}

Un punto di partenza utile per ogni organizzazione è quello relativo all'attuazione di una valutazione delle competenze adattate alle esigenze della specifica istituzione. Tale processo aiuterà nell'identificazione di qualsiasi divario esistente nelle competenze e permetterà di prendere delle decisioni aggiornate sulla formazione e sulla crescita, nonché di evidenziare, potenzialmente, dei compiti integrativi che potrebbero richiedere nuovo personale o nuove responsabilità (e nuove descrizioni lavorative) per quelli che si trovano già a svolgere l'attività. Oltre ad essere un ottimo punto di partenza per migliorare la crescita del personale, potrebbe 
anche essere utile per incorporare degli elementi del processo all'interno di quelli relativi alla crescita e alla valutazione professionale del personale.

II contesto curricolare DigCurV o la Matrix of Digital Curation Knowledge and Competencies possono rappresentare degli strumenti utili quando si avvia una valutazione delle competenze, in questo caso come risorsa che presenta valori di riferimento. Sarà necessario adattare la valutazione alle attività di crescita del personale e ai processi delle singole organizzazioni, tuttavia si devono considerare i seguenti passaggi:

1. Identificare i ruoli all'interno dell'organizzazione con responsabilità di conservazione digitale. L'analisi dei flussi di lavoro può essere d'aiuto in questo processo, come anche mappare questi ultimi a modelli come l'OAIS Reference Model o il Digital Curation Centre Curation Lifecycle Model.

2. Mappare i ruoli agli obiettivi rilevanti del contesto DigCurV.

3. Lavorare con profili specifici per mappare le competenze agli obiettivi rilevanti. Ciò può essere ottenuto in diversi modi tra cui mediante l'autovalutazione e le attività di gruppo. Potrebbe anche essere utile assegnare un punteggio.

4. Analizzare i risultati per individuare scarti, necessità di formazione e altri ruoli richiesti.

\section{Opzioni di Formazione e Sviluppo}

La mancanza di una formazione definita e di opportunità di crescita era considerata in precedenza come un limite significativo per chi volesse avvicinarsi all'ambito della conservazione digitale. Nonostante ci siano ancora difficoltà, anche per chi ha competenze di alto livello, a trovare delle opportunità adatte, ad oggi sono stati istituiti sia corsi brevi che progammi completi per i livelli che vanno da principiante a intermedio che comprendono diverse opportunità di formazione rivolte ad aree specifiche di interesse. Oggi rappresentano un limite il tempo e la spesa che comporta la partecipazione in presenza ai corsi di formazione, anche se si stanno offrendo sempre di più delle alternative di apprendimento a distanza in modo che questo impedimento possa sparire nel tempo.

I corsi di conservazione digitale sono stati spesso criticati in passato soprattutto riguardo al fatto che si dava più risalto alla teoria che alla pratica. Anche questo aspetto sta cambiando, vengono offerte più attività pratiche e demo di strumenti che sono inseriti come parte della formazione. La conservazione digitale comunque rimane una disciplina nella quale molto può essere appreso dalla pratica, le attività di apprendimento tra colleghi e la propensione a sporcarsi le mani possono indurre ad avere risultati migliori. La condivisione delle informazioni e brevi scambi di personale con organizzazioni affini possono fornire un metodo particolarmente efficace per la crescita e l'apprendimento del personale. 


\section{Risorse}



\section{DPOE Training Needs Assessment Survey}

http://www.digitalpreservation.gov/education/2014 Survey Report-Final.pdf

Un'analisi sullo stato attuale delle procedure di conservazione digitale e della capacità di conservare il contenuto digitale delle organizzazioni degli Stati Uniti con lo scopo di definire divari esistenti ed esigenze formative. (13 pagine).

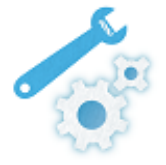

\section{DigCurV, A Curriculum Framework for Digital Curation}

http://www.digcurv.gla.ac.uk/

II DigCurV Curriculum Framework fornisce uno strumento per identificare, valutare e pianificare una formazione che possa soddisfare i requisiti del personale impegnato nella conservazione digitale. II team DigCurV ha portato avanti una ricerca multinazionale nel settore del Patrimonio Culturale per capire quali siano le competenze di chi lavora nella conservazione digitale, e quali siano le figure richieste dai datori di lavoro in questo settore. II framework definisce uno spettro di competenze separate per far combaciare le specifiche esigenze di tre diversi tipi di destinatari: Executives, Managers, e Practitioners.

- Le competenze definite con Executive Lens consentono una conservazione digitale professionale per il mantenimento di una visione strategica.

- Le competenze definite con Manager Lens permettono a un professionista di pianificare e monitorare l'esecuzione dei progetti di conservazione digitale, di scegliere e supportare i team di progetto e di coordinarsi con una serie di figure interne ed esterne al settore del patrimonio culturale.

- Le competenze definite con Practitioner Lens permettono a un professionista di pianificare e portare avanti una serie di attività tecniche, sia individualmente che come parte di un team interdisciplinare.

\section{Matrix of Digital Curation Knowledge and Competencies}

http://ils.unc.edu/digccurr/digccurr-matrix.html

II progetto DigCCurr (Preserving Access to Our Digital Future: Building an International Digital Curation Curriculum) ha realizzato una matrice a 6 dimensioni per l'identificazione e l'organizzazione del materiale che deve essere trattato in un percorso di curation digitale.

\section{Digital Preservation Outreach and Education}

http://www.digitalpreservation.gov/education/curriculum.html 
La linea di partenza del programma di formazione sulla conservazione digitale della Library of Congress per gli archivisti e il personale addetto alla gestione delle collezioni. II corso completo è rivolto agli archivisti e ad altre figure professionali nel campo della conservazione digitale con un approccio basato sul concetto del 'formare il preparatore', in modo da supportare una futura disseminazione del sapere all'interno del gruppo dei colleghi. I video sono disponibili online.

\section{DPC Training}

https://www.dpconline.org/knowledge-base/training

Un ruolo che viene considerato importante per la DPC è legato al potenziamento e allo sviluppo della forza-lavoro dei suoi membri. Per far ciò, la DPC favorisce la formazione, supporta attività, crea risorse mirate ai professionisti del campo ed eventi per tutto l'anno. Questi comprendono: DPC Leadership Programme, Digital Preservation Roadshow, e Member Briefing Days and Invitational Events.

\section{Digital Preservation Training Programme (DPTP)}

https://dptp.london.ac.uk/

Una serie di corsi di formazione sulla conservazione digitale nel Regno Unito. I corsi del DPTP sono organizzati in due o tre giornate e si svolgono regolarmente durante tutto l'anno.

\section{Digital Preservation Management: Implementing Short-Term Strategies for Long-Term} Solutions

http://dpworkshop.org/

Un ottimo tutorial gratuito disponibile online che introduce ai principi di base della conservazione digitale. Particolarmente indicato per bibliotecari, archivisti, curatori, direttori, ed esperti tecnici. Comprende definizioni, concetti chiave, raccomandazioni pratiche, esercizi, riferimenti aggiornati. Il tutorial è disponibile in inglese, francese e italiano.

\section{UK University post-graduate degree courses}

http://www.dpconline.org/knowledge-base/training

La DPC gestisce una lista che sarà utile per chiunque sia interessato a lauree specialistiche con un focus sulla conservazione digitale. Comprende corsi universitari in presenza nel campus o forme di apprendimento a distanza. Alcune università offrono anche dei crediti formativi sulle tematiche riferite alla conservazione digitale.

\section{Education and Training in Audio-visual Archiving and Preservation http://www.arsc-audio.org/etresources.html}

Opportunità formative in Australia, Europa e USA per chi lavora con risorse di suono e immagini in movimento.

\section{Connecting to Collections: Caring for Audio-visual Material}

http://www.connectingtocollections.org/av/

Corso di autoformazione che comprende webinar registrati, dispense, proiezioni, e letture aggiuntive consigliate per singoli studenti che si occupano di risorse audiovisive. Tratta i principi 
di base, una storia dei formati e delle sfide per la loro conservazione, un'identificazione dei formati, gli aspetti legati all'accesso e una panoramica degli standard e dei modelli esistenti. È scritto in inglese da un team di archivisti americani, conservatori, ed esperti di conservazione digitale.

How the DPC makes a difference to your staff

https://vimeo.com/45433968

Brevi interviste con 5 candidati su finanziamento della Digital Preservation Coalition per partecipare alla Digital Futures Academy a Londra nel Marzo del 2012. Discutono della loro esperienza e di come la partecipazione alla DPC abbia portato vantaggi alle loro organizzazioni. (2 minuti 40 secondi) 


\section{Standard e buone pratiche}

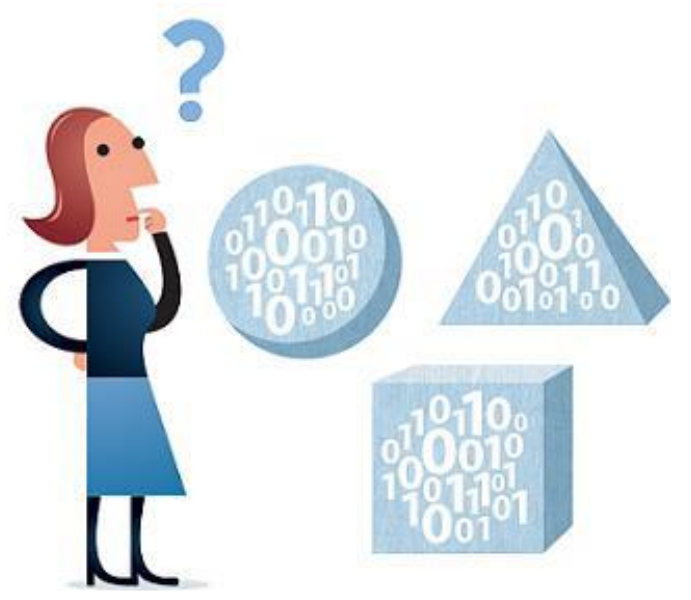

Illustrazione di Jørgen Stamp digitalbevaring.dk CC BY 2.5 Denmark

\section{Introduzione}

L'utilizzo e lo sviluppo di standard affidabili hanno per molto tempo rappresentato un caposaldo nell'industria dell'informazione. Si facilita l'accesso, la scoperta e la condivisione di risorse digitali, come anche la conservazione a lungo termine. Esistono sia standard generici applicabili a tutti i settori che possono supportare le attività di conservazione digitale che standard specifici per contesti industriali, per i quali potrebbe essere previsto un processo di adattamento. L'uso degli standard pertinenti per il contesto istituzionale digitale è di supporto alla conformità organizzativa e all'interoperabilità tra diversi sistemi sia all'interno e che oltre il settore d'interesse. L'aderenza agli standard permette anche alle organizzazioni di essere valutate e certificate.

\section{Standard Operativi}

Ci sono diversi standard che supportano lo sviluppo di un modello operativo per la conservazione digitale.

Mantenere il controllo della custodia delle risorse digitali prevede una serie di procedure per gestire la loro migrazione verso un ambiente di conservazione digitale. Ciò può includere l'identificazione e la quantificazione delle risorse che devono essere trasferite, valutando i costi per conservarle e identificando le esigenze relative alla futura autenticazione e confidenzialità.

ISO 20652: Space Data and Information Transfer Systems - Producer-Archive Interface Methodology Abstract Standard (ISO, 2006) è uno standard internazionale che fornisce un quadro metodologico per la creazione di procedure per il passaggio formale delle risorse digitali dal creatore all'ambiente di conservazione digitale. Gli obiettivi, le azioni e i risultati attesi vengono individuati in quattro fasi - negoziazioni iniziali con il creatore (Fase preliminare), definizione delle necessità (Fase di definizione formale), attraverso il passaggio delle risorse digitali nell'ambiente di conservazione digitale (Fase del passaggio) e con l'assicurarsi che le 
risorse digitali e i loro metadati di accompagnamento siano in linea con quanto concordato (Fase di valutazione).

ISO 14721:2012 Space Data and Information Transfer Systems - Open Archival Information System - Reference Model (OAIS) (ISO, 2012b) fornisce un quadro sistematico per la comprensione e per l'implementazione dei concetti riferiti a un archivio necessari per la conservazione delle informazioni digitali a lungo termine e per il loro accesso, e per la descrizione e la comparazione di architetture e operazioni di archivi esistenti e futuri. Lo standard descrive i ruoli, i processi e i metodi per la conservazione nel lungo periodo. OAIS è stato creato dalla Consultative Committee for Space Data Systems (CCSDS) e venne per la prima volta pubblicato nel 1999, esercitò grande influenza su molti sviluppi nella conservazione digitale sin dagli inizi del 2000. Una guida introduttiva utile allo standard è disponibile come DPC Technology Watch Report (Lavoie, 2014).

Un OAIS è 'un archivio che consiste in un'organizzazione fatta di persone e sistemi che ha accettato la responsabilità di conservare le informazioni e renderle disponibili per una 'Comunità designata' definita'. Un 'archivio OAIS' potrebbe distinguersi da altri usi del termine 'archivio' perché esso accetta e risponde a una serie di specifiche responsabilità. OAIS definisce queste responsabilità come:

- Negoziare e accettare informazioni adeguate dai produttori dell'informazione;

- Riuscire ad avere un controllo sufficiente dell'informazione per raggiungere obiettivi di conservazione a lungo termine;

- Definire l'ambito della comunità di utenti dell'archivio;

- Assicurarsi che le informazioni conservate siano comprensibili in forma indipendente per la comunità di utenti, nel senso che le informazioni possono essere comprese dagli utenti senza il supporto del produttore di queste ultime;

- Seguire delle politiche e strategie documentate per far sì che l'informazione venga conservata a dispetto di ogni ragionevole eventualità, e che non si verifichino cancellazioni ad hoc.

- Rendere l'informazione conservata disponibile per la comunità di utenti, e permettere la diffusione di copie autenticate dell'informazione conservata nella sua forma originale, 0 in una forma che può essere riconducibile all'originale.

L'OAIS identifica inoltre il modello dell'informazione che si deve adottare, che include non solo le risorse digitali, ma anche i metadati usati per descrivere o gestire le risorse e qualsiasi altra informazione di supporto chiamata Rappresentazione delle Informazioni.

II modello funzionale OAIS è ampiamente utilizzato per la determinazione di flussi di lavoro e implementazioni tecniche. Esso definisce un'ampia serie di funzioni di conservazione digitale che includono l'ingest, l'accesso, l'archiviazione, il piano di conservazione, la gestione dei dati e l'amministrazione. Queste offrono un insieme comune di concetti e definizioni che possono facilitare la discussione tra i settori e gruppi professionali, e agevolare la specificazione degli archivi e dei sistemi di conservazione digitale. 
L'OAIS fornisce una struttura di alto livello e un linguaggio utile condiviso per la conservazione digitale, ma per molti anni è stato difficile inquadrare il concetto di 'conformità all'OAIS'. Sebbene il termine sia stato frequentemente utilizzato negli anni immediatamente successivi alla pubblicazione dello standard, si è basato sulla capacità di potersi misurare solo con le sei responsabilità obbligatorie e di alto livello. Un approfondimento sulla 'conformità a OAIS' può essere trovato nel Technology Watch Report.

ISO/TR 18492:2005 Long-term preservation of electronic document-based information (ISO/TR, 2005) offre una metodologia pratica per la conservazione continua e per il recupero di informazioni elettroniche autentiche basate sui documenti, tra cui una guida sull'aggiornamento dei media, sulla migrazione, sulla qualità, sulla sicurezza e sul controllo ambientale. La guida è stata sviluppata per garantire l'autenticità dei record oltre il ciclo di vita dei sistemi originali di conservazione delle informazioni.

ISO 15489:2001 Information and documentation -- Records management (ISO, 2001) può anch'esso rappresentare uno standard utile per la definizione di compiti, processi e metodi per l'implementazione della conservazione digitale laddove l'interesse sia sulla gestione dei record sul lungo periodo. Questo standard riporta il contesto delle buone pratiche per la gestione dei documenti aziendali per assicurare che essi siano curati e documentati per tutto il loro ciclo di vita rimanendo autorevoli e accessibili.

ISO 16175:2011 Principles and functional requirements for records in electronic office environments $(\underline{\text { ISO }, 2011})$ si riferisce ai sistemi di gestione documentale così come ai sistemi di gestione del contenuto di imprese. Anche se non comprende delle necessità specifiche per la conservazione digitale, riconosce però il bisogno di mantenere dei record nel tempo e che le questioni riferite all'obsolescenza del formato debbano essere tenute in considerazione nella specificazione di questi sistemi elettronici.

Esistono anche altri standard internazionali che si occupano più in generale della gestione aziendale e che potrebbero essere pertinenti alla conservazione digitale.

- Certificazione ISO 9001 Quality management systems (ISO, 2015) dimostra quanto un'organizzazione sappia offrire e migliorare prodotti e servizi consistenti.

- Certificazione ISO/IEC 27001 Information technology -- Security techniques -Information security management systems (ISO/IEC, 2013) evidenzia che le risorse digitali sono gestite in modo sicuro garantendone l'autenticità, affidabilità e usabilità.

- ISO/IEC 15408 The Common Criteria for Information Technology Security Evaluation (ISO/IEC, 2009) fornisce una struttura standardizzata per la specificazione di requisiti funzionali e di garanzia per la sicurezza IT e una valutazione rigorosa di questi.

Ci sono diversi modi per certificare l'implementazione della conservazione digitale. Si va dai metodi di certificazione light touch peer review, come ad esempio il Sigillo di approvazione dei dati, passando per metodi interni più completi del DIN 31644 Information and documentation Criteria for trustworthy digital archives (DIN, 2012), fino all'esaustivo standard internazionale 
ISO 16363:2012 Audit and certification of trustworthy digital repositories (ISO, 2012a) (si veda Audit e certificazione).

\section{Standard Tecnici}

Gli standard presentano dei vantaggi in riferimento agli aspetti pratici di un programma di conservazione digitale, principalmente rispetto ai metadati e ai formati dei file.

Insieme agli standard descrittivi riferiti ai metadati, PREMIS e METS di fatto sono standard che miglioreranno un programma di conservazione digitale. PREMIS (PREservation Metadata: Implementation Strategies) è uno standard ospitato dalla Library of Congress e venne pubblicato per la prima volta nel 2005. II dizionario dei dati e gli strumenti di supporto sono stati specificatamente sviluppati per supportare la conservazione di risorse digitali. METS (Metadata Encoding and Transmission Standard) è uno standard con codifica XML che fa in modo che le risorse digitali siano confezionate con informazioni d'archivio (si veda Metadati e documentazione).

Ci sono anche degli standard relativi ai formati dei file. Scegliere i formati dei file non proprietari e basati su standard di formati aperti fa sì che un'organizzazione abbia delle buone basi per un programma di conservazione digitale. ISO/IEC 26300-1:2015 Open Document Format for Office Applications (ISO/IEC, 2015) fornisce uno schema XML per la conservazione di documenti ampiamente utilizzati, come ad esempio documenti testuali, fogli di lavoro, presentazioni. ISO 19005 Electronic document file format for long-term preservation (ISO, 2005) stabilisce gli elementi validi per un PDF/A che assicura che questi siano autonomi e si mostrino in modo consistente sui diversi dispositivi.

Anche gli aspetti relativi a JPEG-2000 e TIFF sono trattati dagli standard ISO. (si veda Formati dei file e standard).

\section{Ostacoli nell'utilizzo degli standard}

Un approccio basato sugli standard per la conservazione digitale è importante, ma esistono anche dei fattori che ostacolano il loro utilizzo come strategia di conservazione digitale, tra cui:

- II ritmo del cambiamento è così veloce che gli standard già formalmente adottati - un processo che solitamente prevede anni - saranno inevitabilmente indietro rispetto agli sviluppi e potrebbero persino essere sostituiti.

- Le pressioni competitive tra fornitori stimolano lo sviluppo di estensioni proprietarie, o le implementazioni degli standard che possono ridimensionare i vantaggi sulla consistenza e sull'interoperabilità della conservazione.

- Gli standard stessi si adattano e cambiano rispetto a nuovi contesti tecnologici, portando a diversi cambiamenti dello standard originale che potrebbe o non potrebbe essere interoperabile nel lungo periodo anche se retrocompatibile nel breve periodo.

- La lettura degli standard può risultare difficile e la loro implementazione richiederebbe molte risorse.

- In un contesto così variabile e distribuito, la regolamentazione non sempre è possibile da seguire. 
Questi fattori implicano che gli standard dovranno essere visti come parte di un pacchetto di strategie di conservazione piuttosto che come strategia principale.

L'ambiente digitale non tende ad essere limitato da regole rigide e un programma di conservazione digitale può spesso essere una combinazione di standard e buone pratiche sufficientemente flessibili e adattate per conformarsi meglio alle necessità dell'organizzazione, alle sue situazioni specifiche e alle risorse digitali che vengono gestite.

\section{Standard, migliori pratiche e buone pratiche}

Negli ultimi anni la guida relativa alle migliori pratiche e ai casi di studio è stata pubblicata da archivi nazionali, biblioteche nazionali e altre organizzazioni culturali. La conservazione digitale è un argomento ben trattato anche all'interno di blog e social media che spesso forniscono informazioni in tempo reale relativamente alla teoria e alla pratica provenienti da tutto il mondo. Gli articoli delle conferenze come iPres, l'International Digital Curation Conference (IDCC) e il Preservation and Archiving Special Interest Group (PASIG) possono rappresentare delle risorse utili per ricevere aggiornamenti nel campo accademico e da professionisti nella conservazione digitale.

Gli standard dovrebbero essere concepiti come una descrizione formale e di ricognizione di ciò che una comunità di esperti considererebbe come migliori pratiche. Gli standard, e le migliori pratiche da cui essi derivano, possono intimorire e c'è il rischio per chi inizia l'attività nella conservazione digitale che 'le cose migliori diventino nemiche di quelle buone'. Perciò, nell'adottare o raccomandare gli standard dovrebbe essere sempre chiaro che alcune azioni sono quasi sempre migliori dell'inattività. La conservazione digitale è un business complicato che fa emergere sfide inattese. Dunque, è quasi sempre vero che sono preferibili gli standard mal implementati rispetto all'attesa di raggiungere la perfezione.

\section{Requisiti per Settori Specifici}

Alcune industrie sono diventate attive nello sviluppo di standard per la conservazione all'interno di un contesto in cui nascono nuovi e particolari tipi di contenuti e casi di studio che si sovrappongono e aumentano il numero degli standard. II fatto che gli standard di conservazione digitale siano incorporati negli standard di settori specifici porta con sé dei benefici per il loro impiego, ma potrebbe rappresentare una sfida per il coordinamento delle attività. Tre esempi:

1. Le Risorse audiovisive rappresentano un caso particolare di conservazione digitale (si veda Immagini in movimento e suono). Le raccomandazioni relative alle registrazioni audio e video vengono fornitr nell'ambito dell'International Association of Sound and Audio-visual Archives (come ad esempio IASA- TC04, 2009), mentre una serie di organismi industriali e detentori di contenuti tra cui la BBC, RAI, ORF e INA hanno creato la PrestoCenter per far andare avanti la ricerca e lo sviluppo degli standard di conservazione in questo campo. https://www.prestocentre.org/

2. L'industria aerospaziale prevede dei requisiti particolari per la gestione del ciclo di vita dei prodotti e degli scambi informativi che hanno dato vita a una serie di grandi iniziative industriali per standardizzare gli approcci al fine di allineare e condividere i disegni CAD 
per l'ingegneria. I membri PROSTEP hanno creato l'ISO 10303 'Standard for Exchange of Product Model Data' riprodotto poi nello standard LOTAR (http://www.lotarinternational.org/lotar-standard/overview-on-parts.html). LOTAR non è incompatibile con OAIS, ma visto che rientra nel protocollo di scambio di dati importanti per l'industria, gli ingegneri aerospaziali sono più predisposti a usare LOTAR invece che OAIS.

3. Anche la Storage Network Industry Association ha iniziato a fare progressi nella creazione di una serie di standard. Un gruppo di lavoro SNIA sulla conservazione dei dati sul lungo periodo è responsabile sia della conservazione fisica e logica, sia della creazione di architetture di riferimento, di servizi e di interfacce per la conservazione. Inoltre, un gruppo di lavoro sull'archiviazione cloud potrebbe diventare particolarmente influente per la conservazione. Le architetture cloud cambiano il modo in cui le organizzazioni considerano i repository e come queste ultime accedono ai servizi per la loro gestione. Ad esempio, non è molto chiaro come si dovrebbe misurare il successo di un repository digitale affidabile che era basato su un fornitore cloud.

\section{Risorse}



Seeing Standards; A visualisation of the metadata universe

http://iennriley.com/metadatamap/

Nell'ambito del patrimonio culturale aumenta sempre più il numero degli standard sui metadati, così come delle loro interrelazioni. Questa mappa visiva del panorama relativo ai metadati vuole supportare coloro che pianificano la selezione e l'implementazione di standard sui metadati. Ognuno dei 105 standard elencati è valutato sulla base della possibilità che venga applicato a categorie specifiche in ognuno dei seguenti quattro assi: comunità, dominio, funzione e scopo. (2010, 1 pagina).

\section{Dlib Magazine}

http://www.dlib.org/dlib.html

Dlib Magazine pubblica regolarmente diversi articoli e casi di studio sull'implementazione pratica di standard e buone pratiche sulla conservazione digitale.

WWW

Core Trust Seal

https://www.coretrustseal.org/

\section{PREMIS}

http://www.loc.gov/standards/premis/

Library of Congress, 2015 


\section{The Digital Curation Centre}

\section{http://www.dcc.ac.uk/}

II Digital Curation Centre rende disponibili le ricerche e i casi di studio riferiti alla conservazione di dati della ricerca. Pubblica anche le registrazioni dei proceedings delle conferenze internazionali che organizza annualmente sulla conservazione digitale.

\section{The Signal}

http://blogs.loc.gov/digitalpreservation/

The Signal è un blog sulla conservazione digitale pubblicato dalla Library of Congress.

\section{IPRES}

http://www.ipres-conference.org/

IPRES, la Conferenza Internazionale sulla conservazione digitale, pubblica un sito web e i proceedings dell'evento annuale che si interessa di diverse tematiche nell'ambito della conservazione digitale.

\section{The Digital Preservation Coalition Wiki}

http://wiki.dpconline.org/index.php?title=Main Page

Wiki della Digital Preservation Coalition che offre uno spazio collaborativo per gli utenti di OAIS, delle valutazioni del formato dei file della British Library, e altre risorse.

\section{Digital Preservation Matters}

\section{http://preservationmatters.blogspot.co.uk/}

II blog Digital Preservation Matters è un account personale di esperienze relative alla Conservazione Digitale.

\section{Riferimenti}

DIN, 2012. DIN 31644 Information and documentation - Criteria for trustworthy digital archives.

Disponibile al seguente link: http://data-archive.ac.uk/curate/trusted-digitalrepositories/standards-of-trust?index $=3$

IASA-TC04, 2009. Guidelines in the Production and Preservation of Digital Audio Objects: standards, recommended practices, and strategies: 2nd edition, edited by Kevin Bradley. Disponibile al seguente link: http://www.iasa-web.org/tc04/publication-information

ISO, 2001. ISO 15489:2001 Information and documentation -- Records management. Geneva: International Organization for Standardization. Disponibile al seguente link: http://www.iso.org/iso/home/store/catalogue tc/catalogue detail.htm?csnumber=31908

ISO, 2005. ISO 19005-1:2005. Document management -- Electronic document file format for long-term preservation. Geneva: International Organization for Standardization. Disponibile al seguente link:

http://www.iso.org/iso/home/store/catalogue tc/catalogue detail.htm?csnumber=38920 
ISO, 2006. ISO 20652:2006 Space Data and Information Transfer Systems - Producer-Archive Interface - Methodology Abstract Standard. Geneva: International Organization for Standardization. Disponibile al link: http://www.iso.org/iso/iso catalogue/catalogue tc/catalogue detail.htm?csnumber=39577

ISO, 2011. ISO 16175:2011 Principles and functional requirements for records in electronic office environments. Geneva: International Organization for Standardization. Disponibile al seguente link:

http://www.iso.org/iso/home/store/catalogue tc/catalogue detail.htm?csnumber=55791

ISO, 2012a. ISO 16363:2012 Audit and certification of trustworthy digital repositories. Geneva: International Organization for Standardization. Disponibile al seguente link: http://www.iso.org/iso/home/store/catalogue tc/catalogue detail.htm?csnumber=56510

ISO, 2012b. ISO 14721:2012 Space Data and Information Transfer Systems - Open Archival Information System (OAIS) - Reference Model. Geneva: International Organization for Standardization. Disponibile al link: http://www.iso.org/iso/home/store/catalogue tc/catalogue detail.htm?csnumber=57284

ISO, 2015. ISO 9001:2015 Quality management systems. Geneva: International Organization for Standardization. Disponibile al seguente link: http://www.iso.org/iso/home/store/catalogue tc/catalogue detail.htm?csnumber=62085

ISO/IEC, 2009. ISO/IEC 15408:2009 The Common Criteria for Information Technology Security Evaluation. Geneva: International Organization for Standardization. Disponibile al seguente link: http://www.iso.org/iso/home/store/catalogue tc/catalogue detail.htm?csnumber=50341

ISO/IEC, 2013. ISO/IEC 27001:2013 Information technology -- Security techniques -Information security management systems. Geneva: International Organization for Standardization. Disponibile al link: http://www.iso.org/iso/home/store/catalogue tc/catalogue detail.htm?csnumber=54534

ISO/IEC, 2015. ISO/IEC 26300-1:2015 Information technology -- Open Document Format for Office Applications (OpenDocument) v1.2 -- Part 1: OpenDocument Schema. Geneva: International Organization for Standardization. Disponibile al seguente link: http://www.iso.org/iso/home/store/catalogue ics/catalogue detail ics. htm?csnumber=66363

ISO/TR, 2005. ISO/TR 18492:2005 Long-term preservation of electronic document-based information. Geneva: International Organization for Standardization. Disponibile al seguente link: http://www.iso.org/iso/home/store/catalogue tc/catalogue detail.htm?csnumber=38716

Lavoie, B., 2014. The Open Archival Information System (OAIS) Reference Model: Introductory Guide (2nd Edition). DPC Technology Watch Report 14-02. Disponibile al seguente link: http://dx.doi.org/10.7207/twr14-02 
Library of Congress, 2015. METS Metadata Encoding and Transmission Standard. Disponibile al seguente link: http://www.loc.gov/standards/mets/ 


\section{Casi aziendali, benefici, costi e impatto}

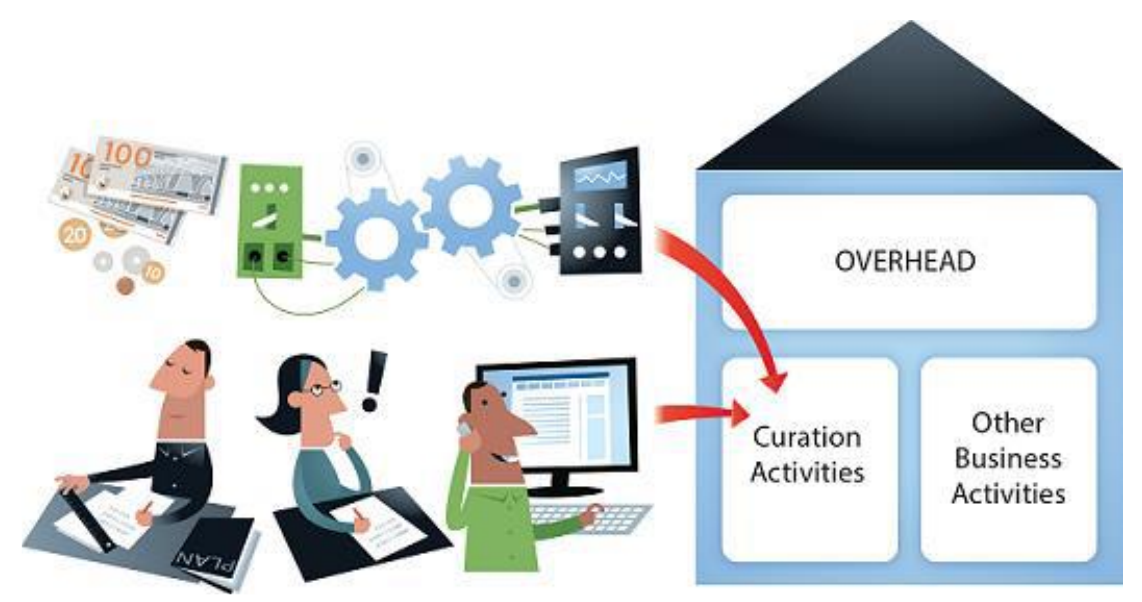

Illustrazione di Jørgen Stamp digitalbevaring.dk CC BY 2.5 Denmark

\section{Introduzione}

Ogni cambiamento che avviene all'interno del contesto economico può far sì che ciascuna organizzazione sia chiamata a ridurre la spesa complessiva e a massimizzare i rendimenti. Allo stesso tempo, le organizzazioni stanno conservando volumi sempre più grandi di risorse digitali. II riutilizzo di modelli può essere parte della risposta a questa sfida. La gestione nel lungo periodo - conservazione - delle risorse digitali è un'attività costosa e complessa, non può essere portata avanti in modo attendibile senza investimenti.

Le sfide per un'organizzazione sono quelle relative alla creazione di modelli aziendali che:

- Possano aiutare a definire i benefici e i risultati, e convincere i principali decisori che si tratta di uno sforzo conveniente;

- Supportino le finalità o gli obiettivi più generali dell'organizzazione produttrice;

- Diano, nel futuro, supporto economico-sostenibile alle loro risorse digitali e aiutino a conciliare le differenze tra gli impegni di investimento/cicli di investimento nel breve e nel medio periodo e gli obiettivi di conservazione nel lungo periodo.

Ci sono organizzazioni che hanno già creato dei modelli per i casi aziendali e per il calcolo dei costi e benefici, per cui il riutilizzo di alcuni o di parti di questi modelli non solo può fare risparmiare del tempo, ma può considerarsi una motivazione per l'adozione di strategie specifiche.

\section{Casi aziendali}

II caso aziendale è uno strumento che supporta e assicura che un investimento venga motivato in termini di direzione strategica dell'organizzazione e di vantaggi che esso apporta. Generalmente, fornisce un contesto, dei vantaggi, dei costi e una serie di opzioni per i decisori principali e per i finanziatori. Può anche definire la modalità con cui valutare il successo per far sì che vengano raggiunti i miglioramenti attesi. 
È fondamentale che qualsiasi modello aziendale o proposta supporti le finalità e gli obiettivi più generali dell'organizzazione produttrice. Allo stesso modo, è importante che i principali stakeholder, come i titolari dei bilanci, vengano consultati e diano delle prospettive preliminari sui programmi e sulla possibilità di fornire commenti e contributi. Un'anticipata presentazione dei programmi può mitigare, per certi versi, quelle situazioni in cui i piani potrebbero altrimenti essere totalmente rifiutati.

Tuttavia, presentare un caso aziendale per la conservazione di qualsiasi risorsa in forma anticipata non garantisce che esso venga accettato. Sebbene non ci sia un modello definito, alcuni o tutti dei seguenti passaggi potrebbero risultare utili qualora il piano venisse rifiutato. All'interno di un'organizzazione potrebbe esserci un insieme di procedure e politiche che riguardano la realizzazione e la presentazione di casi aziendali che dovrebbero essere seguiti. Una comunicazione anticipata di pianificazione aziendale potrebbe essere d'aiuto per l'identificazione di tematiche o aree che potrebbero presentare dei problemi nel momento in cui viene formalmente presentato il piano.

\section{Identificare le opzioni ed essere pragmatici}

Il punto su cui si basa la pianificazione aziendale è rappresentato dall'ambizione e dalla realizzazione di servizi e prodotti che abbiano un valore e dei vantaggi. Non tutti intravedono dei benefici nella conservazione sul lungo periodo in cui i costi rappresentano una questione costante o le risorse devono essere impegnate per il lungo periodo. La pianificazione aziendale è spesso un esercizio di pragmatismo. Potrebbe essere più efficace generare una serie di più piani aziendali a dimensioni ridotte e mirati piuttosto che una singola grande proposta. L'autore del piano aziendale, grazie alla conoscenza di un'organizzazione deve garantire che ogni piano sia realistico e che rientri negli strumenti dell'organizzazione. La pianificazione strategica offre un contesto entro cui i piani aziendali vengono stilati. Qualsiasi obiettivo strategico può essere raggiunto in diverse modalità, ad esempio con meno denaro ma più tempo, personale più ridotto ma un lasso di tempo più lungo, ecc. Una risposta pragmatica offre ai decisori l'opzione migliore e la ragione per cui la si preferisce insieme ad alcune opzioni alternative nel caso aziendale. Può essere anche utile includere 'costi/svantaggi dell'inattività' come un'opzione rispetto alla quale si possono valutare altre azioni.

Se non si hanno esiti positivi all'inizio

Collaborate con gli stakeholder per individuare le ragioni per cui il piano aziendale è stato rifiutato. Parlate con le persone coinvolte nel processo decisionale e cercate un riscontro specifico. L'elemento di costo era troppo caro? I piani erano troppo ambiziosi? II caso aziendale è stato considerato mal compilato o mal presentato? II lasso di tempo non rientrava nei piani organizzativi?

Risposta: Collaborate con gli stakeholder per occuparvi delle questioni principali. Siate chiari nel trattare ogni aspetto. Spiegate le ragioni per cui è stato presentato un piano aziendale e quali sono gli obiettivi che si prefigge di raggiungere. Concentratevi sui benefici, specialmente quelli che riguardano i principali obiettivi strategici dell'organizzazione produttrice. Concentratevi 
sui benefici del piano aziendale sia sul breve che sul più lungo periodo. Un approccio è quello di generare dei piani aziendali che siano 'SMART', cioè Specific, Measurable, Achievable, Realistic e Timely.

\section{Quando cambiano le circostanze}

La parte difficile nella pianificazione aziendale è arrivare al punto in cui il piano è stato accettato. Tuttavia, le circostanze possono cambiare. Se un piano aziendale non è implementato o se i finanziamenti precedentemente previsti vengono ritirati, le implicazioni possono essere gravi. Per cui, si ribadisce che le comunicazioni con gli stakeholder principali sono fondamentali e possono rivelare il perché qualcosa è cambiato.

Risposta: Parte della pianificazione aziendale implica il fatto di disporre di un insieme di opzioni che possono venire offerte nel momento in cui possono sorgere dei problemi relativamente ai finanziamenti di un'opzione prescelta. Disporre di un piano aziendale ben strutturato da cui le proposte possono essere eliminate può aiutare a creare un piano alternativo per implementazioni progressive e diverse che necessitano di meno risorse. In questo caso, un piano aziendale potrebbe essere rapidamente redatto ancora una volta in termini più accettabili e le risorse essere rese disponibili. Concentrarsi sul perché non erano state rese disponibili offre l'opportunità per un caso aziendale di essere nuovamente presentato dando più risalto ai benefici e all'impatto positivo.

\section{Creazione di casi aziendali}

Quando si redige e si consegna un piano aziendale si dovrebbero tenere in considerazione i seguenti passaggi:

\begin{tabular}{|c|l|}
\hline \multicolumn{2}{|l|}{ Creazione di un caso aziendale } \\
\hline $\begin{array}{l}\text { 1. Valutare le proprie risorse digitali e } \\
\text { dare la priorità al lavoro richiesto }\end{array}$ & $\begin{array}{l}\text { Valutare le proprie risorse digitali. Analizzare i } \\
\text { rischi e le opportunità per le proprie risorse } \\
\text { digitali. Utilizzare le proprie analisi per dare } \\
\text { priorità alle aree di lavoro e assegnare loro dei } \\
\text { responsabili. }\end{array}$ \\
\hline 2. È il momento giusto? & $\begin{array}{l}\text { Cosa si sta già facendo? È il momento giusto per } \\
\text { avviare nuove attività per conto proprio? Eे } \\
\text { possibile collaborare con altri? }\end{array}$ \\
\hline 3. Analisi istituzionale & $\begin{array}{l}\text { In che termini è pronta l'istituzione per dei } \\
\text { cambiamenti relativi ai contenuti e ai processi? }\end{array}$ \\
\hline Analisi degli stakeholder e advocacy & $\begin{array}{l}\text { Chi se ne occuperà e chi utilizzerà le risorse } \\
\text { digitali? Chi prende le decisioni sui } \\
\text { finanziamenti? È bene impegnarsi con questi } \\
\text { ultimi usando un linguaggio e dei termini che } \\
\text { comprenderanno. }\end{array}$ \\
\hline
\end{tabular}




\begin{tabular}{|c|l|}
\hline $\begin{array}{l}\text { 5. Obiettivi: ambito degli obiettivi, } \\
\text { attività, piano e costi }\end{array}$ & $\begin{array}{l}\text { Mappare quello che si farà, chi farà cosa, quanto } \\
\text { costerà, e quando avverrà. }\end{array}$ \\
\hline $\begin{array}{l}\text { 6. Mappare i benefici alla strategia } \\
\text { organizzativa }\end{array}$ & $\begin{array}{l}\text { Assicurarsi di rendere espliciti i vantaggi del } \\
\text { proprio caso aziendale in un modo comprensibile } \\
\text { per i propri finanziatori. }\end{array}$ \\
\hline 7. Cos'altro è necessario? & $\begin{array}{l}\text { Si deve aggiungere un'analisi costi-benefici o } \\
\text { una lista di opzioni basate sulle spese e i risultati? }\end{array}$ \\
\hline $\begin{array}{l}\text { 8. Validare e perfezionare il caso } \\
\text { aziendale }\end{array}$ & $\begin{array}{l}\text { Riesaminare e testare il proprio piano aziendale } \\
\text { rispetto alle buone pratiche e individuare cos'altro } \\
\text { è necessario. }\end{array}$ \\
\hline $\begin{array}{l}\text { 9. Consegnare il piano aziendale } \\
\text { 10. Condividere un caso aziendale } \\
\text { modificato }\end{array}$ & $\begin{array}{l}\text { Si dispone di un campione da usare } \\
\text { nell'organizzazione? Ricordarsi che è possibile } \\
\text { doverlo realizzare nuovamente. }\end{array}$ \\
\hline
\end{tabular}

Per un modello di caso aziendale per la conservazione digitale e per ulteriori informazioni si veda il Digital Preservation Business Case Toolkit.

\section{Benefici}

I benefici sono associati ai costi e anche ai rischi (si veda Gestione del rischio e del cambiamento). Se i rischi vengono mitigati possono diventare un tipo di benefici. II fine dell'acquisizione di qualsiasi risorsa digitale è che queste ultime vengano utilizzate. Gli usi che si fanno delle risorse digitali rappresentano un vantaggio per gli utenti. Se un'organizzazione ha bisogno di comprendere i costi associati alle risorse digitali, essa dovrà prendere in considerazione anche i benefici. I benefici possono essere utilizzati per giustificare i costi attraverso le creazioni di piani aziendali.

Calcolare i vantaggi spesso rappresenta una sfida, in particolare quando questi benefici non sono facilmente decifrabili in termini quantitativi. Spesso verrà richiesta una combinazione di approcci per l'analisi sia qualitativa che quantitativa dei risultati e per presentare le differenze che sono state create. Per supportare le istituzioni, il progetto Keeping Research Data Safe ha creato un KRDS Benefits Framework e un Benefits Analysis Toolkit (KRDS, 2011). Questi hanno come fine quello di aiutare le istituzioni a identificare la portata complessiva dei benefici a partire dalla gestione e conservazione dei dati della ricerca fino alla loro presentazione in modo sintetico a un gruppo di diversi stakeholder (ad es., quando si crea un caso aziendale o attività di sostegno). Il toolikit è anche facilmente applicabile ai benefici della conservazione digitale per gli altri tipi delle risorse digitali. 


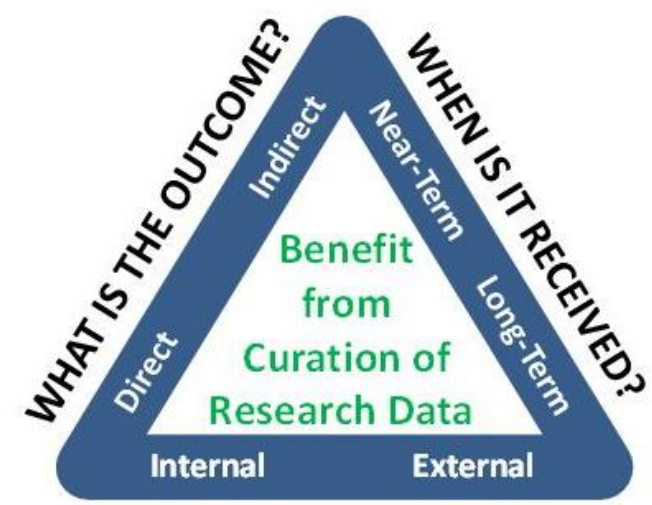

WHO BENEFITS?

II KRDS Benefits Framework utilizza tre dimensioni per far luce sui vantaggi che possono essere potenzialmente generati dagli investimenti. Queste dimensioni funzionano come una struttura ad alto livello entro cui i benefici possono essere organizzati e modellati su valori più mirati attraverso l'utilizzo del Toolkit. Aiuta a individuare quali cambiamenti si stiano cercando di attuare, quali i risultati, chi ne beneficia, e quanto tempo sarà necessario per realizzare questi benefici.

\section{Costi}

Un caso aziendale di regola non solo guarderà ai costi di elaborazione per le soluzioni di conservazione digitale, ma ai costi complessivi tra cui la gestione dei costi di progetti/programmi e altre attività che si sono intraprese per supportare l'implementazione, come quella relativa alla formazione e alla pubblicità. Tuttavia, i costi di conservazione digitale rappresentano spesso un elemento critico.

\section{Perché comprendere i costi della conservazione digitale?}

Esistono diverse motivazioni sul perché un'organizzazione potrebbe voler valutare i costi di conservazione digitale:

- Pianificare e mettere in bilancio per costruire da zero un nuovo repository o per ampliarne uno esistente.

- Aggiungere una nuova risorsa digitale al proprio repository e decidere se può essere sostenuta nell'immediato o sul lungo periodo.

- Offrire una piattaforma per la comparazione con le organizzazioni tipo e un'opportunità di utilizzare dei rendimenti che sono stati individuati da altri.

- Decidere se esternalizzare le attività o svolgerle all'interno.

- Decidere quanto investire per fornire un servizio di conservazione digitale ai clienti.

- Capire dove vengono usate le risorse o se non sono sufficientemente utilizzate, e le aree in cui il posizionamento di risorse aggiuntive potrebbe recare dei vantaggi. 
Cos'è la modellazione del costo del ciclo di vita?

Diversi progetti di ricerca e sviluppo hanno voluto modellare i costi di conservazione digitale lungo il ciclo di vita dalla creazione e ingest fino alla conservazione, e infine all'accesso. Un ampio numero di progetti fa luce su questo lavoro presentando quali sono i risultati più applicabili a situazioni specifiche, scegliendo un modello e mettendo in pratica una sfida importante. II 4C Project ha indagato, analizzato e valutato questo lavoro e offre una guida su come ottenerne il massimo:

- Starting out with curation costs - offre un'introduzione ai concetti.

- Using cost models - descrive il modo in cui selezionare un modello di costo adeguato alla propria organizzazione.

- Cost Concept Model and Gateway Specification - offre più dettagli tra cui una guida per generare un modello rispetto ai propri bisogni in riferimento a concetti come 'rischio', 'valore', 'qualità' e 'sostenibilità'.

\section{Sfide nella modellazione del costo}

La modellazione del costo è vista come un'attività particolarmente difficile che presenta una serie di aspetti complessi, tra cui:

- Definizione dei driver o delle finalità per i costi della conservazione digitale.

- La conservazione digitale è un target in cambiamento, determinato da tecnologie in cambiamento e necessità istituzionali in evoluzione.

- Livello del dettaglio: a livelli alti, la modellizzazione diviene meno utile poiché generalmente si riferisce a una sezione trasversale di diversi contesti di conservazione. Modellare su livello basso diventa rapidamente molto complesso, rendendo i modelli difficili da generare, mantenere e mettere in atto.

- Le organizzazioni sono restie a condividere i dati di contabilità con cui i modelli potrebbero essere creati e validati.

- Persino laddove siano stati condivisi i dati di contabilità, spesso è difficile mappare tra i diversi modelli con cui essi sono associati.

- La separazione dei costi di conservazione digitale da altri costi aziendali è complicata e a volta inutile (esempio: digitalizzare le collezioni in un modo che aiuti l'ingest del contenuto all'interno della conservazione - si tratta di conservazione digitale o di digitalizzazione dei costi?).

Per questa ragione, modellare i costi di conservazione digitale lungo il ciclo di vita rappresenta un'attività che dovrebbe essere trattata con attenzione. Modellare sarà sempre un'approssimazione, perciò si dovrà definire la quantità di tempo da dedicarvi per riuscire ad ottenere un risultato quanto meno approssimativo.

\section{Gestione dei costi}

È possibile gestire i costi mediante un'attenta pianificazione. Uno dei modi per farlo è rappresentato da una buona progettazione dei processi. Le modalità con cui vengono create 0 acquisite, gestite e diffuse le risorse digitali, comportano dei costi che dipendono dall'organizzazione e possono essere gestiti. II processo end-to-end dall'acquisizione alla 
diffusione deve essere progettato in un modo tale da minimizzare la necessità di risorse mentre si massimizza l'efficienza. Se i rendimenti lavorano meglio su scale, un processo efficiente non deve essere un processo a grandi dimensioni. L'automatizzazione di passaggi sistematici può far anche risparmiare del tempo e creare dei processi consistenti efficaci. I costi iniziali della progettazione e implementazione di processi possono essere controbilanciati da ritorni sul periodo più lungo.

\section{Impatto}

Generalmente, si considera l'impatto come calcolo dei benefici, riferiti in particolar modo a un pubblico e società più generali e ottenuti dopo la realizzazione di un progetto di caso aziendale.

Per progetti a dimensioni più ridotte e casi aziendali, l'impatto potrebbe consistere solamente in un semplice insieme di misure come i download o il numero di richieste di siti web rispetto alle quali si può facilmente valutare il successo.

Per i progetti e programmi di dimensioni più grandi, l'impatto può essere parte di una valutazione più complessiva al fine di giustificare le risorse spese. Può comprendere un mix di misure quantitative e qualitative e di solito può essere effettuato da specialisti esterni che lavorano con il personale del repository. Questi utilizzano dei metodi specifici dell'ambito economico, della gestione e dell'informatica, per esempio l'analisi dei costi-benefici o analisi contingenti, e metodi tradizionali di scienze sociali come ad esempio interviste, indagini e focus group.

Le misurazioni spesso implicano la scelta di metriche o indici e prevedono un'attenta pianificazione e accordo su cosa misurare e come misurare. Le metriche spesso utilizzano elementi facilmente misurabili come ad esempio download, scale metriche non sono propriamente numeriche, scale di valutazione o categorie di variabili. Generalmente, esiste una relazione inversa tra ciò che idealmente dovrebbe essere misurato (ad esempio, utenti e utilizzo) e approssimazioni che sono facili da acquisire e misurare (ad esempio, "visitatori unici" e download web).

\section{Risorse}

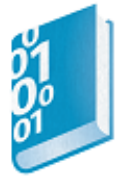

Sustainable Economics for a Digital Planet: Ensuring Long-Term Access to Digital Information

\section{http://brtf.sdsc.edu/biblio/BRTF Final Report.pdf}

La Blue Ribbon Task Force ha indagato sulla conservazione digitale sostenibile e sull'accesso da un punto di vista economico. II report finale identifica i problemi intrinseci a tutte le risorse digitali conservate, e propone delle azioni che possono intraprendere gli stakeholder per affrontare le sfide legate alla sostenibilità. Redige agende operative che sono rivolte ai gruppi 
maggiori di stakeholder e a strategie di conservazione digitale per specifici domini. (2010, 116 pagine).

The Value and Impact of Data Sharing and Curation: A synthesis of three recent studies of UK research data centres

http://repository.jisc.ac.uk/5568/1/iDF308 -

Digital Infrastructure Directions Report\%2C Jan14 v1-04.pdf

Questa sintesi riepiloga e riflette sui risultati complessivi di una serie di indagini indipendenti sul valore e sull'impatto di tre noti centri di dati della ricerca o servizi britannici (Economic and Social Data Service, Archaeology Data Service, e British Atmospheric Data Centre). Gli approcci utilizzati in questi studi investigano sul valore e sugli impatti dei servizi di dati della ricerca e la condivisione e archiviazione dei dati che essi hanno concesso. La collezione di dati impiegata è incentrata sulle indagini relative all'utente e depositante, e ai dati finanziari e operativi dei centri di dati (ad es., registrazione utente, depositi di dataset e download), accompagnati da interviste di approfondimento. Non tutti gli impatti vengono rilevati e quantificati, per cui questi approcci economici sono stati affiancati da altri, come il KRDS Benefits Framework, per mostrare i vantaggi più generali. (2014, 26 pagine).

Jisc Guide: Digitising your collections sustainably

https://www.jisc.ac.uk/guides/digitising-your-collections-sustainably

Presenta un punto di partenza per la considerazione degli aspetti necessari alla creazione di un modello aziendale che farà da supporto alla digitalizzazione e alle collezioni digitali.

\section{C Project Collaboration to Clarify the Costs of Curation https://www.4cproject.eu/}

L'Unione Europea ha finanziato il progetto 4C per aiutare le organizzazioni in Europa a investire in modo più efficace nella conservazione e curation digitale. Sono stati realizzati diversi report e risorse che sono disponibili nella pagina del progetto outputs and deliverables. Questi comprendono il Digital Curation Sustainability Model, Evaluation of Cost Models and Needs \& Gaps Analysis, Report on Risk, Benefit, Impact and Value, e Draft Economic Sustainability Reference Model.

La valutazione del report sui modelli di costo analizza dieci modelli di costo che includono KRDS e LIFE. Un altro risultato rilevante è il Curation Costs Exchange (CCEx), una piattaforma di proprietà collettiva che aiuta le organizzazioni di qualsiasi tipo nella valutazione dei costi delle attività di curation attraverso metodi comparativi e analitici. La CCEx ha lo scopo di fornire una formazione veritiera sui costi per facilitare la creazione di investimenti aggiornati sulla conservazione digitale. La CCEx è stata pubblicata nel 2014 da 4C e adesso è gestita e diretta dalla Digital Preservation Coalition (DPC) con l'aiuto del NESTOR e della Netherlands Coalition for Digital Preservation (NCDD). 


\section{Digital Preservation Business Case Toolkit}

http://wiki.dpconline.org/index.php?title=Digital Preservation Business Case Toolkit

Questo Toolkit offre una guida approfondita per redigere un piano aziendale incentrato sulle attività di conservazione digitale. Rivolto ai professionisti (e ai loro direttori) che lavorano con risorse digitali e che vorrebbero ottenere finanziamenti per sviluppare le loro attività nella conservazione digitale. Si rivolge principalmente a coloro che cercano altri finanziamenti all'interno delle loro organizzazioni, ma potrebbe anche dare delle informazioni utili a chi propone fondi di progetto da parte di un ente finanziatore esterno. Comprende una guida passo dopo passo per la costruzione di un caso aziendale e di un Template per questa realizzazione. Creato da JISC e finanziato da SPRUCE Project nel 2013, il wiki del toolkit è ospitato dalla DPC.

\section{Keeping Research Data Safe (KRDS) Benefits Toolkit}

http://www.beagrie.com/krds/

Keeping Research Data Safe (KRDS) sono studi sui costi/benefici, strumenti e metodologie che si concentrano sulle sfide presenti nella valutazione dei costi e benefici della conservazione e curation dei dati della ricerca. Sebbene il focus sia sui dati della ricerca, gli strumenti possono essere facilmente personalizzati per essere utilizzati in diverse aree della conservazione digitale. I risultati che sono disponibili comprendono KRDS Factsheet, KRDS User Guide, KRDS Activity Cost Model, e KRDS Benefits Analysis Toolkit, così come materiali supplementari e report. I progetti KRDS tra il 2008 e 2011 sono stati finanziati da JISC.

\section{Cost Questions for Digital Preservation}

http://www.metaarchive.org/public/publishing/ma 20costquestions final.pdf?thumblink

The MetaArchive Cooperative ha realizzato un set di 20 domande per 'supportare gli istituti nella Ioro analisi comparativa di diverse soluzioni di conservazione digitale'. Questo lavoro segna un passaggio dallo sviluppo di modelli di costo predittivi dettagliati verso un approccio più generale che cerca di identificare e capire i principali driver di costo piuttosto che i costi effettivi stessi.

\section{DSHR's Blog}

http://blog.dshr.org/search/label/storage\%20costs

http://blog.dshr.org/search/label/cloud\%20economics

David Rosenthal è un blogger che tratta di frequente l'argomento relativo ai costi di archiviazione, spesso considerando l'impatto dell'evoluzione delle tecnologie di archiviazione sui costi di conservazione e sull'archiviazione cloud.

\section{A Digital Asset Sustainability and Preservation Cost Bibliography}

http://blogs.loc.gov/digitalpreservation/2012/06/a-digital-asset-sustainability-and-preservation-

\section{cost-bibliography/}

Una serie di riferimenti bibliografici che 'spaziano ampiamente dagli articoli sulla "valutazione contingente", "valutazione dell'ecosistema" e i "costi" generali della conoscenza, per chi si occupa direttamente di aspetti legati ai costi associati alla conservazione digitale e all'amministrazione'. 


\section{Digital Preservation and Data Curation Costing and Cost Modelling}

http://wiki.opf-labs.org/display/CDP/Home

Una lista di modelli di costo della conservazione digitale e di iniziative sul modellamento dei costi.

\section{The Cost of Inaction Calculator Rationale}

\section{https://coi.weareavp.com/rationale}

È un ottimo video informativo di AVPreserv sul costo dell'inattività e sulla logica del caso aziendale per la conservazione digitale. Si concentra sul materiale audiovisivo, ma vale la pena ascoltare e riflettere sulla logica sottostante qualsiasi tipo di risorsa digitale si possieda. (8 minuti 41 secondi)

\section{Casi di studio}

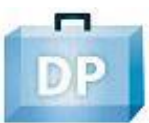

\section{KRDS Benefits Toolkit case studies}

Ci sono 4 casi di studio che forniscono esempi pratici di schede di lavoro complete dei partner di progetto:

\section{Archaeology}

http://www.ukoln.ac.uk/events/i2s2-krds/presentations/catherine-hardiman-krds-benefit-

framework-2011-07-v2.ppt

II background di questo caso di studio viene offerto dalla presentazione del workshop di disseminazione Archaeology Data Service (ADS). Sono disponibili esempi pratici dell'ADS Benefits Framework Worksheet (PDF) ADS Value-chain and Impact Worksheet (Excel 972003).

\section{Health: Population Cohort Studies}

http://www.ukoln.ac.uk/events/i2s2-krds/presentations/dipak-kalra-krds-benefits-2011-07.ppt II background di questo caso di studio viene offerto dalla presentazione del workshop di disseminazione Medical Research Council Cohort Studies. Come esempio pratico, vi è quello del Cohort Studies Value-chain and Impact Worksheet (Excel 97-2003).

\section{Research Data Citation: SageCite}

http://www.ukoln.ac.uk/events/i2s2-krds/presentations/monica-duke-krds-sagecite-benefits2011-07.ppt 
II background di questo caso di studio viene offerto dalla presentazione del workshop di disseminazione SageCite. Come esempio pratico, vi è quello del SageCite Benefits Framework Worksheet (PDF).

\section{Social Sciences: UK Data Archive (UKDA)}

http://www.ukoln.ac.uk/events/i2s2-krds/presentations/matthew-woollard-krds-benefits-2011-

\section{7.ppt}

II background di questo caso di studio viene offerto dalla presentazione del workshop di disseminazione UKDA. Come esempio pratico, vi è quello del UKDA Benefits Impact Worksheet (PDF).

Digital Preservation Business Case Toolkit

Ci sono quattro casi di studio provenienti dalle attività portate avanti come parte dello SPRUCE Project Awards:

\section{Bishopsgate library case study}

http://wiki.dpconline.org/index.php?title=Bishopsgate library case study

Un audit di collezioni e un caso aziendale incentrato sui primi passi nell'ambito della conservazione digitale.

\section{Institute of education case study}

http://wiki.dpconline.org/index.php?title=Institute of education case study

Una riesamina dell'approccio e della generazione del caso aziendale per il mantenimento dei documenti digitali amministrativi.

\section{Northumberland estates case study}

http://wiki.dpconline.org/index.php?title=Northumberland estates case study

Valutazione delle soluzioni di repository digitale e un caso aziendale associato per la conservazione digitale.

\section{Lovebytes case study}

http://wiki.dpconline.org/index.php?title=Lovebytes case study

Un trial di stabilizzazione dei media e della conservazione del contenuto insieme a un caso aziendale nella prospettiva di un passaggio a uno status produttivo.

\section{Riferimenti}

Keeping Research Data Safe (KRDS), 2011. Digital Preservation Benefits Analysis Toolkit. Accessibile al link: http://beagrie.com/krds-i2s2.php 


\section{Attività organizzative}

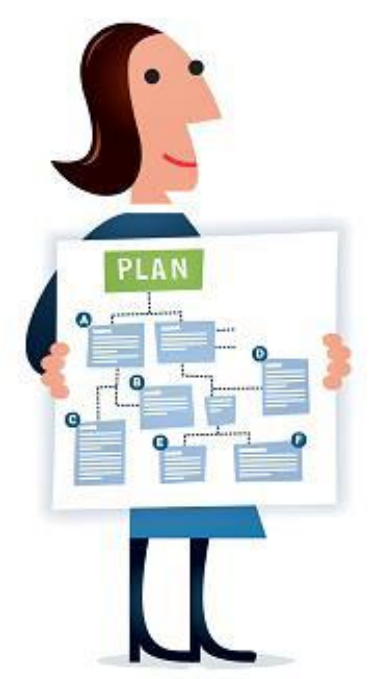

Illustrazione di Jørgen Stamp digitalbevaring.dk CC BY 2.5 Denmark

\section{A chi è rivolto?}

Ai creatori ed editori delle risorse digitali, fornitori di servizi di parti terze, dirigenti (DigCurV Manager Lens) e personale (DigCurV Practitioner Lens), responsabili dell'implementazione di attività istituzionali di rilevanza per la conservazione digitale. Si suppone che tra questi vi sia a) personale proveniente da parti dell'organizzazione separate e b) un'ampia gamma di conoscenze relative alla conservazione digitale, dal livello principiante al livello avanzato; c) prospettive sia tecniche che non tecniche; d) un'ampia serie di attività funzionali con collegamenti diretti o indiretti alle attività di conservazione digitale.

\section{Requisiti minimi di conoscenza}

Ad ampio raggio: da principianti ad avanzati.

\section{Obiettivi}

- Fornire dei riferimenti per le fonti attendibili e una guida che mira a favorire l'uso di atteggiamenti corretti nella creazione e gestione di risorse digitali. L'importanza riferita alla figura del creatore nel facilitare il processo di conservazione digitale viene più volte evidenziata all'interno del Manuale, ma in particolare nella sezione Creazione delle risorse digitali. Le buone pratiche nella digitalizzazione e nella creazione di risorse digitali sono essenziali per disporre di una fattibilità costante delle risorse digitali.

- Accrescere la consapevolezza sui fattori che devono essere tenuti in conto quando si creano o si acquisiscono le risorse digitali.

- Fornire dei riferimenti alle fonti utili attendibili e una guida sia per utenti principianti che per quelli che hanno già iniziato a considerare le implicazioni della tecnologia digitale sulle loro attività operative. 


\section{Creazione di risorse digitali}

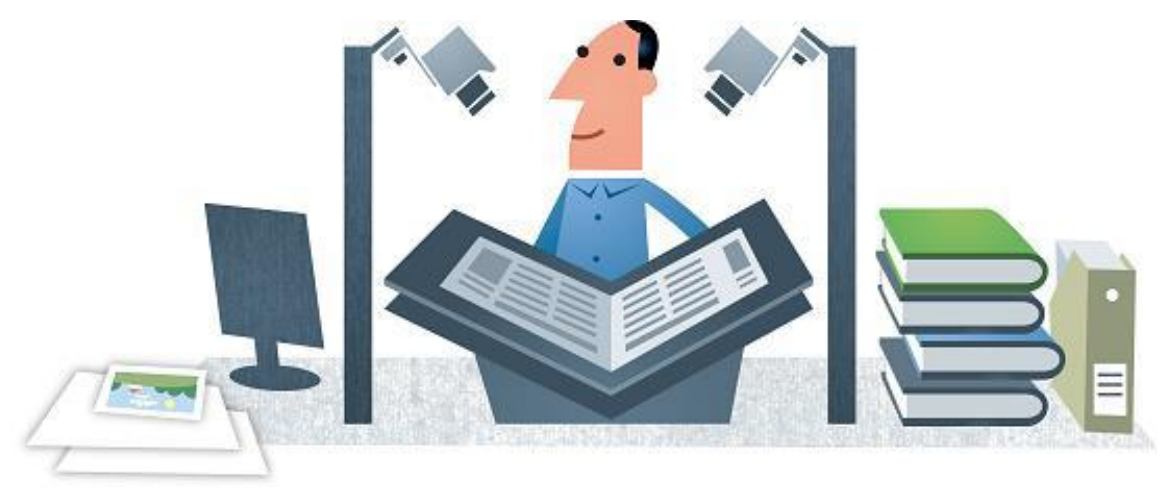

Illustrazione di Jørgen Stamp digitalbevaring.dk CC BY 2.5 Denmark

\section{Introduzione}

"The first line of defense against loss of valuable digital information rests with the creators, providers and owners of digital information." (Waters and Garrett, 1996)

La Task Force on Archiving of Digital Information ha prima fra tutte sottolineato il ruolo fondamentale rivestito dai creatori nell'aiuto che danno per garantire l'accesso nel lungo periodo alle risorse digitali create. Da allora questa prospettiva è stata ripresa più volte in molti altri documenti.

Ovviamente, non ci si aspetta che la maggior parte dei creatori sia impegnata a lungo termine nella conservazione del contenuto digitale creato al di là delle loro esigenze aziendali. Ogni risorsa digitale ha un ciclo di vita che comprende/include diversi stakeholder e interessi. Tuttavia, nell'ambito della conservazione nel lungo periodo, il dialogo tra i repository a lungo termine e i creatori è un aspetto auspicabile e realizzabile. Ed è spesso nell'interesse del creatore stesso che il contenuto generato sia ben strutturato, completo, corretto e utilizzabile per obiettivi esistenti e futuri.

II creatore dei contenuti digitali riveste un ruolo importante nel processo di attuazione della conservazione nel breve e nel medio periodo, spesso per un intervallo equivalente a decenni, e per lo meno nell'agevolare la conservazione dal medio al lungo periodo, risulta fondamentale esortare gli utenti ad assumere comportamenti corretti (e anche sostenuti dai repositories).

Questa sezione si concentrerà solamente su comportamenti corretti da adottare nella creazione di risorse digitali che saranno di supporto per la loro longevità di utilizzo attivo, gestione futura e conservazione. Per le relative attività e guida si consultino le altre sezioni pertinenti del Manuale.

II nostro focus rimane legato alle implicazioni generali della conservazione digitale nel processo di digitalizzazione (copie digitali) o quello delle risorse native digitali. 
La creazione di risorse native digitali o di tipi di file nativi digitali specifici di dominio, come ad esempio documenti elettronici, dati della ricerca, o informazioni personali digitali, disporrà di eccellenti fonti di ulteriore assistenza e guida. I riferimenti principali sono all'interno della sezione Risorse e casi di studio.

\section{Creazione di risorse native digitali}

La conservazione digitale si occupa di una serie di attività che sono indispensabili per garantire un accesso alle risorse digitali costante per tutto il tempo necessario. Tra queste attività ci sono quelle comprese nella creazione di risorse native digitali necessarie per venire incontro ad esigenze attuali del creatore originale.

Spesso, molte di queste attività necessarie per l'accesso continuativo nel lungo periodo vanno a sovrapporsi a quelle buone pratiche adattate alle necessità aziendali immediate. Infatti, molte organizzazioni e singoli individui creano risorse digitali ora e avranno bisogno di utilizzarle per molti anni. Probabilmente non considererebbero quello che stanno facendo come un'attività di conservazione digitale, e altri termini come 'continuità digitale' sono usati di frequente per identificare l'effetto che queste attività hanno su di loro che non sono istituti di conservazione della memoria come musei, biblioteche, o archivi.

È importante che i creatori capiscano che se non lavorano attivamente per assicurare continuità, le loro risorse digitali possono divenire facilmente inutilizzabili. Si tratta di assicurarsi che le informazioni siano complete, disponibili, e, quindi, utilizzabili per i bisogni aziendali.

Le proprie informazioni saranno utilizzabili se si è in grado di:

- Trovarle quando se ne ha bisogno;

- Aprirle quando se ne ha bisogno;

- Lavorare con esse nel modo necessario;

- Capire cosa sono e di cosa trattano;

- Fidarsi che le cose stiano come le stesse affermano.

Ciò permette di lavorare in modo responsabile, legale, effettivo ed efficace. Aiuta a proteggere la propria reputazione, prendere delle decisioni consapevoli, ridurre i costi e produrre dei servizi migliori. Per altre informazioni sui primi passi nella conservazione digitale si veda la sezione Guida introduttiva.

La tabella di seguito offre un orientamento sugli aspetti principali e sulle azioni da considerare quando si creano le risorse digitali al fine di assicurarne la longevità dell'utilizzo attivo e potenziale per una conservazione a lungo termine. 


\section{Conservare le risorse native digitali}

\section{Software e formati}

Scegliere il software meglio supportato e che crei file che possano essere letti da diversi programmi.

Per una guida si veda la sezione del Manuale Formati dei file e standard.

\section{$2 \quad$ Nomi dei file}

Usare un nome dei file corto e descrittivo riferito al contenuto e a una data che fornisca un contesto e che possa essere facilmente compresa sia da utenti che da computer nel momento presente e nel futuro.

Non usare spazi o caratteri speciali (a parte - o__), ciò eviterà possibili fraintendimenti da parte del sistema hardware e software del computer.

Porre l'informazione riferita alla data seguendo il formato standard contenuto in ISO 8601:2004, YYYY-MM-DD. Ciò rappresenta una modalità consistente di tracciamento delle versioni. Si tenga a mente che ogni azione successiva può cambiare in automatico i metadati separati riferiti alla data dei file generati dai sistemi.

Usare un metodo consistente per mostrare le versioni dei file: la data, integrata se richiesto con ad esempio un numero di versione, $\mathrm{v} 1$, v2, v_final, etc.

\section{$3 \quad$ Archiviazione e backup}

Per un'ulteriore guida, si vedano i Principi per utilizzare i sistemi di archiviazione IT per la conservazione digitale nella sezione del Manuale Archiviazione.

\section{$4 \quad$ Conoscere i propri obblighi e le buone pratiche rilevanti}

Per una guida al riguardo, si veda la sezione del Manuale Conformità giuridica. Molti obblighi e buone pratiche saranno specifici per progetti o settori - per un esempio si veda l'inserto di seguito Creare Dati della ricerca.

\section{$5 \quad$ Piano per le istituzioni}

Alcune transizioni si possono prevedere e pianificare, altre invece potrebbero essere impreviste, ma comunque mitigate da una buona pianificazione e procedure corrette. Per una guida di riferimento, si vedano di seguito le sezioni del Manuale Risorse e casi di studio, Piano di conservazione e Gestione del rischio e dei cambiamenti.

\section{Creazione di copie digitali}

L'attenzione rivolta alla digitalizzazione in questa sezione riflette la sua importanza concreta dal momento che molte istituzioni si stanno avviando verso la digitalizzazione di parti delle loro collezioni. È importante sottolineare che questo Manuale non sta valutando il potenziale della digitalizzazione come uno strumento di riformattazione della conservazione. L'enfasi è sulla conservazione delle risorse native digitali, o sui prodotti della digitalizzazione (copie digitali), non sulla conservazione degli originali analogici.

Un'eccezione importante è relativa alle risorse audiovisive. Le risorse audio e video necessitano della digitalizzazione per la sopravvivenza del loro contenuto, a fronte dell'obsolescenza del sistema di riproduzione e decadimento e danneggiamento di elementi fisici, che siano analogici o digitali (si veda Immagini in movimento e suono). 
Molti progetti di digitalizzazione hanno come obiettivo principale quello di un accesso migliore, un obiettivo perfettamente legittimo, ma che, a meno che non si presti attenzione alle modalità con cui l'accesso può essere mantenuto nel tempo, potrebbe avere breve vita. È poco probabile che tutte le iniziative relative alla digitalizzazione si stiano attuando con rispetto alla sostenibilità nel lungo periodo delle copie digitali che si stanno creando, perciò è utile favorire l'adozione di buone pratiche nella creazione di risorse digitali e di affidarsi a guide esistenti.

Una buona parte di ciò che ora si sta digitalizzando nasce come contenuto nativo digitale. Poi è stato convertito nel formato analogico come stampa su carta prima che ci fosse bisogno di un accesso digitale e che fosse considerata importante la ridigitalizzazione. Questo ciclo ha bisogno di essere rapidamente cambiato per semplificare la gestione di più contenuti nativi digitali.

\section{Conservare le copie digitali: considerazioni sulla conservazione digitale}

$1 \quad$ Valutazione della necessità di digitalizzare

Le risorse sono state già digitalizzate? Se sì, lo si è fatto in linea con uno standard appropriato $\mathrm{e}$ sono rapidamente accessibili dal proprio pubblico?

\section{Cercare finanziamenti per il progetto}

Che politiche di archiviazione esistono, sia da parte dell'agenzia di finanziamento (se finanziata esternamente) che dell'istituzione principalmente responsabile del progetto?

$3 \quad$ Pianificazione del progetto e attribuzione delle risorse

Necessità di mettere da parte i finanziamenti ricorrenti per il mantenimento delle copie digitali così come i fondi una tantum per la conversione.

Assicurare che tutti gli stakeholder di pertinenza siano al corrente del progetto (ad esempio, se ci si aspetta che un'altra parte dell'organizzazione o un'agenzia esterna mantengano le risorse, queste avranno bisogno di essere coinvolte a un certo punto del processo, se non prima).

Identificare una strategia per portare avanti le attività del progetto in modo sostenibile dopo che questo è arrivato ai suoi deliverables. Questa strategia potrebbe comportare l'inges ${ }^{23}$ delle attività del progetto in un catalogo di collezioni dell'organizzazione produttrice, o nell'incarico di un'istituzione partner per la ricezione di questi asset.

$4 \quad$ Selezione delle risorse

Copyright. Sarà necessario fare in modo che vengano rilasciati permessi sia per digitalizzare gli originali che per fare le copie della copia digitale ai fini della conservazione e rilascio. Per altre informazioni si veda Conformità giuridica.

Condizioni e completezza dell'originale: è in grado di essere ri-scannerizzata in un momento successivo se la copia digitale viene persa?

5 Determinare quanto il contenuto informativo debba essere organizzato

${ }^{23}$ [...] per identificare ciò che i bibliotecari chiamano accessione e gli archivisti acquisizione, ma che nella sostanza coincide in entrambi i casi con l'ingresso di una risorsa informativa nel sistema documentario (OAIS, Digltalia, p. 36) 
(per esempio, database di testo consultabili e/o immagine di pagine di documento)

Selezione dei formati dei file adeguati e di un tipo di archiviazione sia per le copie master/d'archivio che per i derivati, si vedano le sezioni Formati dei file e standard, Metadati e documentazione e Archiviazione.

6 Decidere il metodo di digitalizzazione adeguato all'originale analogico e agli obiettivi del progetto.

Preparare gli originali per la digitalizzazione. I dettagli del metodo di digitalizzazione devono essere documentati e allegati ai record dei metadati per permetterne una gestione futura.

$7 \quad$ Preparare gli originali per la digitalizzazione

II National Archives fornisce degli standard e una guida sulla preparazione dei documenti per la digitalizzazione dei registri (The National Archives, 2015).

Gli originali saranno mantenuti? Non si devono scartare gli originali finché non venga stabilito che a) la versione elettronica sia giuridicamente consentita e/o b) che la versione elettronica permetta una conservazione a lungo termine.

Decidere se mantenere o no gli originali dopo la digitalizzazione di certo non rappresenterà un problema per i progetti che prevedono una digitalizzazione di opere di valore all'interno di una collezione, ma la questione centrale sarà se l'originale è o meno troppo fragile per essere scannerizzato nuovamente in un momento successivo se la copia digitale venisse perduta. In ognuno di questi casi, se la copia digitale diventa il principale mezzo di accesso, sarà soggetta alle stesse disposizioni delle risorse native digitali.

\section{Conversione}

La documentazione delle caratteristiche tecniche.

L'algoritmo di compressione (se utilizzato); un minimo di profondità richiesta; risoluzione della scannerizzazione, ecc. Creare delle copie di backup non appena viene avviata la conversione.

\section{$9 \quad$ Controlli della garanzia di qualità}

II surrogato digitale deve avere una qualità di conservazione accettabile.

Se si usano servizi di parti terze, bisogna assicurarsi che la documentazione chiarisca le responsabilità per la garanzia di qualità.

10 Indicizzazione finale e catalogazione

I metadati per la presentazione delle risorse e per la gestione e conservazione della copia digitale.

11 Caricare i dati nei sistemi informatici

Le richieste di accesso all'archiviazione documentale e alle copie di conservazione (se differenti).

Rendere adatte le copie di backup.

12 Implementare strategie di archiviazione e conservazione o affidarsi a un'agenzia di conservazione

Standard richiesti per i formati, supporti di archiviazione, documentazione, e procedure di trasferimento. Archiviazione di master e copie di backup.

Strategie relative all'aggiornamento dei media e ai cambiamenti avvenuti in ambito tecnologico. 


\section{Risorse}

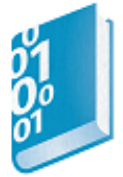

\section{Digitisation at The National Archives}

http://nationalarchives.gov.uk/documents/information-management/digitisation-at-the-nationalarchives.pdf

Questo documento definisce gli standard TNA e i requisiti per la digitalizzazione di documenti analogici nella sua collezione. Viene anche raccomandato ai settori governativi britannici che desiderano digitalizzare qualunque loro record: l'intero processo di digitalizzazione a partire da una scannerizzazione iniziale fino alla riproduzione delle immagini per la conservazione, incluse la specificazione di immagine scannerizzata del National Archives, la scannerizzazione dei record in cui le immagini risultanti diventeranno il documento pubblico a livello legale per la conservazione permanente, e la scannerizzazione dei record in cui le immagini diventeranno delle copie digitali con i record dei paper originali che sono conservati e che rimangono come documento pubblico legale. (Luglio 2015, 56 pagine).

Koninklijke Bibliotheek/National Library of the Netherlands: Metamorfoze preservation imaging guidelines

https://www.metamorfoze.nl/sites/default/files/publicatie documenten/Metamorfoze Preservati on Imaging Guidelines 1.0.pdf

Metamorfoze è il programma nazionale olandese di conservazione del patrimonio documentario. Le linee guida sono redatte per la digitalizzazione di risorse bidimensionali, come manoscritti, archivi, libri, articoli di giornale e riviste. Possono essere anche applicate alle fotografie, ai quadri e ai disegni tecnici. Le linee guida si riferiscono esclusivamente alla qualità dell'immagine e ai metadati del file di conservazione master da cui possono essere generati tutti gli output destinati alla stampa o al web. (2012, 44 pagine).

\section{Preparing Collections for Digitisation}

Questo libro di Anna E. Bulow and Jess Ahmon del 2010 fornisce una guida pratica che riguarda il processo end-to-end della digitalizzazione delle collezioni e può essere usato come un manuale di riferimento, sul modello di 'come fare per', per i direttori delle collezioni che si stanno avviando verso un progetto di digitalizzazione o che si stanno occupando di un progetto esistente. Tratta anche alcuni degli aspetti più generali, come l'uso delle copie per la conservazione, e la sostenibilità a lungo termine dell'accesso digitale. (208 pagine).

\section{InterPARES 2 Creator Guidelines Making and Maintaining Digital Materials}

http://www.interpares.org/ip2/display file.cfm?doc=ip2(pub)creator guidelines booklet.pdf Questo opuscolo fornisce indicazioni per chi crea risorse digitali nel corso delle proprie attività professionali e personali, ed è di supporto per garantire la loro conservazione. (10 pagine). 
InterPARES 2 Preserver Guidelines Preserving Digital Records: Guidelines for Organisations

http://www.interpares.org/public documents/ip2(pub)preserver guidelines booklet.pdf

Questo opuscolo fornisce indicazioni ad ogni organizzazione responsabile per la conservazione a lungo termine dei documenti digitali (10 pagine).

WWW

Jisc Digital Media resources

Intellectual property rights in a digital world

https://www.jisc.ac.uk/guides/intellectual-property-rights-in-a-digital-world

Digitising your collections sustainably

https://www.jisc.ac.uk/guides/digitising-your-collections-sustainably

Federal Agencies Digitization Guidelines Initiative

http://www.digitizationguidelines.gov/

Frutto della collaborazione delle agenzie federali americane per la definizione di linee guida comuni, metodi e procedure per la digitalizzazione di contenuti storici. Ne fanno parte due gruppi di lavoro che stanno studiando gli aspetti specifici di due ambiti principali, Fermo Immagine e Audiovisivo.

\section{Future Proof - Protecting our digital future \\ http://futureproof.records.nsw.gov.aul}

Future Proof è un'iniziativa dello State Records avviata dal New South Wales State Government in Australia. II sito web e il blog riguardano i prodotti e i progetti della State Records che interessano nello specifico i documenti digitali. I link alle categorie forniscono degli accessi utili a diverse risorse che sono all'interno del sito.

\section{The Curation Reference Manual}

http://www.dcc.ac.uk/resources/curation-reference-manual

Questa risorsa gestita dal Digital Curation Centre comprende indicazioni, informazioni approfondite e critiche sulle tecniche attuali della curation digitale e sulle buone pratiche. II Manuale è un progetto in corso collaborativo, che coinvolge i membri della comunità DCC, i quali suggeriscono tematiche, scrivono ogni numero e eseguono revisioni. Ciascun numero è progettato per aiutare i custodi dei dati, i produttori e gli utenti a capire meglio le sfide che affrontano e i ruoli che assumono nella creazione, gestione e conservazione delle informazioni digitali nel tempo.

\section{UK Data Archive: Consent for data sharing}

https://www.ukdataservice.ac.uk/manage-data/legal-ethical/consent-data-sharing

Collezionare, usare e condividere i dati nella ricerca prevede che gli obblighi etici e legali vengano rispettati. Leggi come il Data Protection Act, Freedom of Information Act e Statistics and Registration Services Act gestiscono anch'esse l'utilizzo di alcuni tipi di dati. Questa guida 
offre supporto su come i dati della ricerca possano essere condivisi senza violare alcuna responsabilità etica o giuridica.

\section{An Elevator Pitch for File Naming Conventions}

http://acrl.ala.org/techconnect/post/an-elevator-pitch-for-file-naming-conventions

Questo post sul blog Association of College and Research Libraries (ACRL) TechConnect afferma la necessità di adottare un approccio coerente quando si denominano i file o gli elementi software, dimostrandone gli effetti qualora non lo si faccia (2013).

\section{Digital Continuity guidance from The National Archives UK}

http://www.nationalarchives.gov.uk/information-management/manage-information/policy-

\section{process/digital-continuity/}

Una guida completa sulla continuità digitale del National Archives. Particolarmente utili sono Understanding Digital Continuity, un'introduzione all'argomento (2011, 20 pagine); e Managing Digital Continuity, che descrive un processo a 4 fasi: 1) piano d'azione 2) definizione dei requisiti di continuità digitale. 3) valutazione e gestione dei rischi per la continuità digitale. 4) mantenimento di una continuità digitale.

\section{NCDCR Digital Preservation Best Practices and Guidelines - Create Digital Files} http://digitalpreservation.ncdcr.gov/

Pubblicato per la prima volta nel 2010 da parte del Digital Information Management Program della State Library del North Carolina, e la Digital Services Section dello State Archives del North Carolina, questo sito ha ricevuto nel 2012 il riconoscimento National Digital Stewardship Alliance Innovation Award. Lo scopo è quello di fornire informazioni pratiche e introduttive sulla conservazione digitale e guidare i visitatori alle risorse di volta in volta accessibili.

\section{Digital Preservation Management Tools and Techniques}

\section{http://dpworkshop.org/workshops/management-tools}

I workflow di contenuto del workshop DPM comprendono un diagramma ad alto livello suddiviso in diagrammi di livello inferiore per la gestione del contenuto fisico, per la transizione attraverso la digitalizzazione, e per la gestione del contenuto nativo digitale e digitalizzato. L'idea è di offrire un workflow comune per tutti i contenuti in qualsiasi contesto, e poi sviluppare diversi casi d'uso per mettere in evidenza le eccezioni a contenuti specifici con diversi tipi di requisiti.

\section{Part 1: Why is File Naming Important?}

https://www.youtube.com/watch?v=Hi A4Ywn4VU

Questo breve video è la prima parte di quattro tutorial sulla denominazione dei file. Si concentra sul perché sia importante scegliere oculatamente il nome dei propri file. Rivolto a un pubblico generale, è parte delle serie 'Inform U' della State Library del North Carolina (2012, 3 minuti 19 secondi). 


\section{Casi di studio}

DPC case note: ULCC assessing long term access from short term digitization projects http://www.dpconline.org/component/docman/doc download/534-

\section{casenoteassessingpreservationindigitization.pdf}

I progetti di digitalizzazione sono per lo più finanziati su un periodo di tempo breve, perciò, quali sono le tecniche per rendere solida la digitalizzazione nel lungo periodo? II caso di studio finanziato da Jisc descrive il lavoro intrapreso dalla University of London Computer Centre nell'analisi di piani a lungo termine di 16 progetti di digitalizzazione e fornisce uno strumento investigativo di base per aiutare sia finanziatori che direttori del progetto a riflettere sui loro piani di conservazione a lungo termine. Novembre 2010 (4 pagine).

\section{The British Library 'Save our Sounds' project}

http://www.bl.uk/projects/save-our-sounds

Partito nel 2015, il programma della British Library 'Save our Sound' è volto alla conservazione attraverso la digitalizzazione del Sound Archive nazionale, una collezione di più di 6.5 milioni di registrazioni di discorsi, musica, natura e ambiente, dal 1880 ad oggi. II progetto ha lo scopo di garantire sia una conservazione appropriata dell'archivio esistente che sistemi adatti per l'acquisizione di produzioni sonore future nel Regno Unito.

\section{Digital Curation Centre case studies}

http://www.dcc.ac.uk/resources

Nel 2013 la DCC avviò una serie di casi di studio per integrarli nella nuova guida DCC 'How to Develop Research Data Management Services'. Questi casi di studio riguardano specifici elementi del servizio di Research Data Management d'interesse per i ricercatori e gestori dei dati.

\section{Society of American Archivists campus case studies}

http://www2.archivists.org/publications/epubs/Campus-Case-Studies

I Campus Case Studies sono dei report degli archivisti universitari americani che hanno realizzato soluzioni operative. Trattano una varietà di argomenti, alcuni dei quali specificamente incentrati sulla conservazione digitale e creazione di documenti digitali. L'attualità dei casi di studio varia dal 2008 ad oggi.

\section{Why metadata matters}

\section{https://cbaileymsls.wordpress.com/2013/09/29/metadata/}

Questo post presenta degli esempi utili relativi al perché una scelta non ottimale dei nomi dei file e delle descrizioni di metadati quando si crea un file possa ostacolare le ricerche future, il reperimento delle informazioni e il riutilizzo. 


\section{Riferimenti}

The National Archives, 2015. Digitisation at The National Archives. Disponibile al seguente link: http://nationalarchives.gov.uk/documents/information-management/digitisation-at-the-nationalarchives.pdf

Waters, D and Garrett, J., 1996. Preserving Digital Information: Report of the Task Force on Archiving of Digital Information commissioned by the Commission on Preservation and Access and the Research Libraries Group. Washington, DC: Commission on Preservation and Access. Disponibile al seguente

link:

https://www.oclc.org/content/dam/research/activities/digpresstudy/final-report.pdf 


\section{Acquisizione e valutazione}

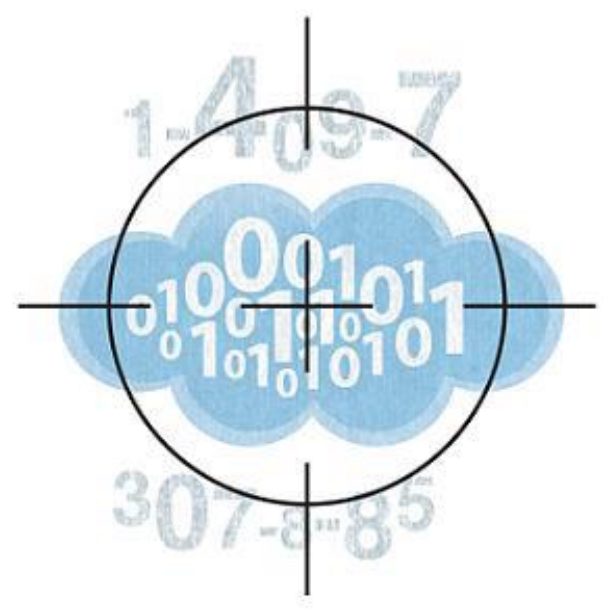

Illustrazione di Jørgen Stamp digitalbevaring.dk CC BY 2.5 Denmark

\section{Introduzione}

In un ambiente digitale le decisioni prese riguardo alla creazione e alla selezione hanno delle implicazioni significative per la conservazione. Il legame tra l'accesso e la conservazione è molto più esplicito rispetto alla carta o altre risorse tradizionali, dal momento che si può perdere l'accesso alle risorse digitali in uno spazio di tempo relativamente breve se non si intraprendono misure attive per il mantenimento (ovvero, conservazione) sin dall'inizio. Come descritto nell'Albero Decisionale interattivo, se non è né fattibile né auspicabile conservare una risorsa digitale a causa dei cambiamenti nella tecnologia, la sua acquisizione dovrebbe essere rivalutata. Mentre molti degli stessi principi specifici del processo di conservazione tradizionale possono essere opportunamente impiegati, le politiche e le procedure avranno bisogno di essere adattate all'ambiente digitale.

Nella stampa le dimensioni fisiche di un archivio implicano delle motivazioni di scelta piuttosto chiare, mentre la decisione di conservare può essere presa abbastanza indipendentemente e entro un arco di tempo di diversi decenni. Al contrario, le risorse digitali possono aumentare e la valutazione può rappresentare un compito arduo. Inoltre, queste risorse possono diventare inaccessibili in tempi relativamente rapidi, per cui le decisioni sulla selezione e conservazione per le collezioni digitali potrebbero dover essere prese simultaneamente.

Se da una parte questo può comportare una selezione delle risorse digitali fatta con maggior rigore rispetto alle risorse su stampa o altre risorse analogiche, dall'altra eviterà i costi che altrimenti si presenterebbero dal momento che la conservazione retrospettiva delle risorse digitali non è un'operazione raccomandata.

Nell'ambiente digitale è anche importante avere una documentazione precisa che non solo fornirà dei dettagli essenziali per la gestione delle risorse nel tempo, ma anche delle informazioni 
sul contesto, senza le quali ci sarebbero poche ragioni per la conservazione dell'oggetto digitale in sé anche se è una operazione tecnicamente fattibile. Nella sezione di accompagnamento Alberi decisionali, si suggerisce che l'acquisizione venga rivalutata qualora la documentazione risultasse inadeguata.

Nel caso di risorse digitali interconnesse, fornire l'accesso a una risorsa non necessariamente prevede che questa possa essere fisicamente inserita nella collezione; il concetto di acquisizione è abbastanza diverso dalle collezioni tradizionali. Ci sono una serie di opzioni disponibili per garantire l'accesso o per costruire delle 'collezioni virtuali'. Per esempio, fare delle copie/accessi speculari fornendo degli hyperlink ad una risorsa, a cataloghi online e cercando supporti.

In alcuni casi, un ente potrebbe essere restio ad assumersi la responsabilità principale per le risorse se percepisce che l'interesse nella loro conservazione non sia così ampiamente condiviso al punto che potrebbe rappresentare un onere eccessivo per le istituzioni. Ciò enfatizza la necessità di stabilire degli accordi equi per degli sforzi condivisi ove opportuno. Negli ultimi anni sono comparsi diversi servizi, come il Keepers Registry per le riviste elettroniche - il loro impegno a conservare le risorse potrebbe essere d'interesse generale. La sezione di accompagnamento Alberi decisionali per la valutazione e la selezione è basata sul concetto che le risorse non sono ancora state acquisite e indica una serie di punti rispetto ai quali dovranno essere tenute in considerazione le implicazioni di costo prima di avviare il processo di acquisizione. Si suggerisce anche che in riferimento a questi punti si potrebbero dover compiere delle scelte difficili per quanto riguarda la validità delle risorse rispetto ai costi 0 la scelta di proseguire con l'acquisizione.

\section{Sviluppo delle politiche e procedure}

Prima di imbarcarsi nell'acquisizione e ingest delle collezioni digitali, potrebbe essere necessario stabilire se le politiche attuali (ad es., lo sviluppo di collezioni) e le procedure siano ancora adeguate agli obiettivi da raggiungere (si veda Politiche e strategie istituzionali). A seconda della struttura e del testo dei documenti le revisioni possono includere qualunque cosa da piccoli cambiamenti per aumentare il campo d'applicazione così da includere gli oggetti digitali fino alla creazione di nuovi documenti strategici che si occupano specificatamente di collezioni digitali. Aggiunte o modifiche alle policy potrebbero comprendere le descrizioni dei tipi di oggetti che saranno acquisiti, in relazione al formato e/o contenuto, così come potrebbero occuparsi di altri aspetti tra cui i diritti di proprietà intellettuale, dati sensibili e considerazioni

sull'accesso. È fondamentale che qualsiasi cambiamento venga sancito da commissioni specifiche di gestione all'interno della propria organizzazione per garantire il supporto e l'acquisto.

\section{Politiche di Valutazione/Conservazione}

La sezione Alberi decisionali che segue può essere utilizzata come strumento per creare o testare la selezione o la valutazione/conservazione della policy della propria organizzazione. 
La valutazione di oggetti nativi digitali dovrebbe comprendere una valutazione accurata del loro valore rispetto all'organizzazione produttrice nel contesto delle sfide della conservazione a lungo termine e della modalità di accesso continuo. Queste sfide possono comprendere la capacità di un'organizzazione di leggere o aprire la versione di un file master, la capacità di rendere sicuri i diritti sufficienti a gestire e garantire l'accesso alle versioni presenti e future dei file, o semplicemente di garantire il personale e le risorse di finanziamento. Dunque, le organizzazioni dovrebbero concentrarsi inizialmente sull'equilibrio tra l'acquisizione di oggetti digitali di grande valore e questi obblighi curatoriali a lungo termine. Si dovrebbe tenere a mente che le organizzazioni possono garantire l'accesso alle risorse che hanno acquisito senza posizionarle in flussi di lavoro specifici di conservazione o mantenimento. Un documento strategico articolato che individua chiaramente le risorse digitali più importanti (sia dal punto di vista del formato che del contenuto) può orientare la valutazione di oggetti digitali destinati per questi percorsi. Per acquisizioni digitali di valore inferiore, che spesso risultano raggruppate insieme ad acquisizioni di valore superiore, potrebbe essere sufficiente delineare il livello di accesso della conservazione che un'organizzazione garantirà loro. Questo scenario dovrebbe comprendere un piano di conservazione adatto a questo tipo di contenuto, e ciò potrebbe significare, ove opportuno, includere anche un piano di scarto o una politica di selezione.

\section{Accordi e guida per i depositari - formati dei file, documentazione richiesta}

Una volta stabilite le policy, diversi documenti di supporto saranno richiesti per agevolare il processo di acquisizione e valutazione. Insieme ai documenti di processo standard, è possibile che un'organizzazione voglia creare una serie di accordi standard a favore di detentori e di licenze per supportare i processi di negoziazione. Ciò sarà particolarmente utile affinché siano garantiti i diritti di proprietà intellettuale necessari per la conservazione e le autorizzazioni minime. Un'organizzazione senza degli accordi di licenza sufficienti potrebbe trovarsi di fronte a delle collezioni digitali di cui non detiene i diritti necessari a preservarle o a garantirne l'accesso (si veda Conformità giuridica). Ci saranno integrazioni di note tecniche per i detentori che stabiliscono i requisiti per le risorse da trasferire e una documentazione di accompagnamento.

\section{Standard per l'acquisizione e trasferimento}

I fatti dimostrano che il trasferimento da un produttore a un archivio può essere tortuoso, e perciò ogni strumento che velocizzi il processo potrebbe essere utile per entrambe le parti. Si sono intraprese due iniziative per standardizzare l'interfaccia tra Produttori e Archivi in un processo consistente e ben concepito, favorendo un'intesa tra produttori e archivi relativamente ai loro rispettivi ruoli: Producer-Archive Interface - Methodology Abstract Standard (PAIMAS, ISO 20652:2006) e il Producer-Archive Interface Standard (PAIS, ISO 20104:2015).

PAIMAS offre una descrizione standardizzata delle interazioni tra i produttori e un archivio, segmenta il processo di trasferimento in diverse fasi, fornendo una descrizione dettagliata dei risultati previsti per ogni step e le azioni necessarie per arrivare a questo risultato. Le quattro fasi principali - Fase Preliminare, Fase della Definizione Formale, Fase di Trasferimento, Fase di Validazione - servono da base per l'identificazione di aree interne all'interfaccia ProduttoreArchivio che trarrebbe benefici da standard più mirati, raccomandazioni, e buone pratiche, e 
forniscono un riferimento per lo sviluppo di processi automatici e strumenti software per supportare il processo di trasferimento delle informazioni.

PAIS fornisce una metodologia standard per definire formalmente gli oggetti informativi digitali che devono essere trasferiti da un Produttore informatico a un Archivio e per confezionare efficacemente questi oggetti sotto forma di Pacchetti di Versamento. Lo scopo è quello di sostenere delle definizioni più precise degli oggetti digitali, supportare i processi degli archivi e validare gli oggetti ricevuti durante la sottomissione.

\section{Workflow di acquisizione}

\section{Negoziazione}

La negoziazione dei termini riferiti al deposito dovrebbe avvenire prima che qualsiasi record sia stato trasferito. Nella politica d'acquisizione dell'organizzazione si dovrebbero trattare molti aspetti dell'accordo di deposito, ma i dettagli a proposito di ogni deposito, specialmente per gli archivi locali e specialistici, potrebbero essere richiesti a livello della collezione. II depositante dovrebbe riportare se ci sono delle limitazioni in riferimento a cosa e quando i record possono venire pubblicati, per esempio: c'è qualche risorsa che può essere immediatamente aperta mentre altre possono esserlo solo alla morte del depositante o dopo un determinato periodo di tempo?

Una considerazione importante è riferita al diritto che l'organizzazione ha nell'alterazione del record a fini di conservazione, ad esempio migrare verso un formato che può essere conservato nel lungo periodo o a cui si può avere accesso.

Se il trasferimento comprende un contenuto che è essenziale alla conoscenza dei record, ma non costituisce esso stesso un record, ci dovrebbe essere un accordo che faccia in modo che l'organizzazione possa rimuovere quei file nel momento in cui viene acquisito il loro contenuto per utilizzarlo altrove (ad esempio, i metadati per i record).

\section{Trasferimento}

Molti enti avranno bisogno di sviluppare delle procedure e dei documenti per sostenere un trasferimento adeguato delle risorse digitali dai fornitori alle loro collezioni.

Quando si spostano gli oggetti nativi digitali in uno spazio IT dell'organizzazione si dovrebbe tenere in considerazione come questa operazione avviene in modo da garantire la sicurezza e la completezza del trasferimento. Per le organizzazioni a dimensioni più ridotte potrebbe bastare avere dei file digitali su un supporto o un sistema simile hardware, e verificare i loro contenuti rispetto al file descrittivo manifest.

In alternativa, le organizzazioni potrebbero voler trasferire i file usando un FTP interno, o una soluzione a pagamento di parti terze (come un cloud basato sul servizio di condivisione dei file), al fine di garantire una catena di custodia. 
La tabella riportata di seguito mostra quali sono le opzioni per il trasferimento e la selezione delle risorse digitali. Le decisioni riguardanti i formati dei file, e se rilevanti dei supporti di archiviazione (si vedano le sezioni Archiviazione, Supporti obsoleti, e Formati dei file e standard), saranno di supporto e interdipendenti con questo processo.

\begin{tabular}{|c|c|c|}
\hline Opzioni & Problema & Requisiti \\
\hline Tutte & & $\begin{array}{l}\text { - Policy su Formati dei file e } \\
\text { standard. } \\
\text { - } \frac{\text { Piano di conservazione e }}{\text { monitoraggio tecnologico }} \\
\text { sugli sviluppi } \\
\text { nell'Archiviazione e sui } \\
\text { formati di Supporti obsoleti. }\end{array}$ \\
\hline $\begin{array}{l}\text { Una serie limitata di } \\
\text { formati dei file ricevuti } \\
\text { Una serie limitata di media } \\
\text { ricevuti (la maggior parte } \\
\text { delle opzioni con rapporto } \\
\text { costo/efficacia a lungo } \\
\text { termine) }\end{array}$ & $\begin{array}{l}\text { - Semplifica la } \\
\text { gestione e riduce i } \\
\text { costi complessivi. } \\
\text { - I detentori } \\
\text { potrebbero non } \\
\text { avere risorse o } \\
\text { competenze a cui } \\
\text { adeguarsi. } \\
\text { - Un'ampia varietà di } \\
\text { formati dei file } \\
\text { impiegati e } \\
\text { estensioni } \\
\text { proprietarie per } \\
\text { standard aperti. } \\
\text { - I supporti di } \\
\text { archiviazione fisici } \\
\text { usati per il } \\
\text { trasferimento } \\
\text { potrebbero essere } \\
\text { dei portatori } \\
\text { temporanei e il } \\
\text { contenuto potrebbe } \\
\text { essere trasferito in } \\
\text { un tipo di } \\
\text { archiviazione nel } \\
\text { lungo periodo. }\end{array}$ & $\begin{array}{l}\text { - Linee guida sui formati dei } \\
\text { file preferiti. } \\
\text { - Livello di influenza sul } \\
\text { deposito. } \\
\text { - Strategie di Sostegno e } \\
\text { Collaborazioni per } \\
\text { raggiungere gli obiettivi } \\
\text { desiderati. } \\
\text { - Linee guida sul } \\
\text { trasferimento dei media } \\
\text { prescelti e delle procedure. }\end{array}$ \\
\hline $\begin{array}{l}\text { Si accettano i formati dei } \\
\text { file per come ricevuti ma si }\end{array}$ & $\begin{array}{l}\text { - Semplifica la } \\
\text { gestione e riduce i } \\
\text { costi complessivi. }\end{array}$ & - $\frac{\text { Conformità giuridica, }}{\text { autorizzazioni di copyright o }}$ \\
\hline
\end{tabular}




\begin{tabular}{|c|c|c|}
\hline $\begin{array}{l}\text { convertono nel formato dei } \\
\text { file standard } \\
\text { Si accettano i supporti di } \\
\text { archiviazione per come } \\
\text { ricevuti ma si trasferiscono } \\
\text { i contenuti all'archivio } \\
\text { standard utilizzato }\end{array}$ & $\begin{array}{l}\text { - La conversione } \\
\text { verso un formato di } \\
\text { file standard } \\
\text { potrebbe non } \\
\text { essere } \\
\text { tecnicamente } \\
\text { fattibile. } \\
\text { - Sarà necessario } \\
\text { controllare che non } \\
\text { si sia verificata una } \\
\text { perdita accidentale } \\
\text { dei dati. }\end{array}$ & $\begin{array}{l}\text { diritti di conservazione } \\
\text { regolati dalla legge. } \\
\text { - Risorse e competenze } \\
\text { tecniche nell'istituto } \\
\text { competente. } \\
\text { - Documentazione dei formati } \\
\text { nativi per permettere la } \\
\text { conversione. } \\
\text { Controlli d'integrità per il } \\
\text { processo di conversione. }\end{array}$ \\
\hline $\begin{array}{lr}\text { Si accettano e si } \\
\text { archiviano per come } \\
\text { ricevuti (opzione meno } \\
\text { economica nel lungo } \\
\text { termine nonostante } & \text { costo } \\
\text { iniziale più basso) } & \end{array}$ & $\begin{array}{l}\text { Complica la } \\
\text { gestione e } \\
\text { aumenta i costi di } \\
\text { gestione delle } \\
\text { risorse nel tempo. } \\
\text { - Opzione ad alto } \\
\text { rischio, in } \\
\text { particolare se si } \\
\text { stanno } \\
\text { raccogliendo } \\
\text { grandi numeri di } \\
\text { risorse digitali. } \\
\text { È possibile } \\
\text { scegliere i formati } \\
\text { dei file. La scelta } \\
\text { potrebbe non } \\
\text { essere la più } \\
\text { adatta per la } \\
\text { conservazione. } \\
\text { La qualità dei } \\
\text { supporti di } \\
\text { archiviazione e } \\
\text { l'adeguatezza per } \\
\text { la conservazione } \\
\text { nel lungo periodo } \\
\text { potrebbero non } \\
\text { essere resi noti. } \\
\text { I formati } \\
\text { potrebbero essere } \\
\text { obsoleti o non } \\
\text { supportati } \\
\text { all'interno dell'ente. }\end{array}$ & $\begin{array}{l}\text { - Priorità chiaramente definite } \\
\text { per la conservazione sia nel } \\
\text { breve che nel lungo periodo. } \\
\text { - Capacità di occuparsi di } \\
\text { argomenti come la } \\
\text { decrittazione, software } \\
\text { proprietari, ecc., in relazione } \\
\text { agli oggetti ricevuti. } \\
\text { - Capacità di assicurare un } \\
\text { accesso futuro alle } \\
\text { informazioni contenute } \\
\text { nell'oggetto. }\end{array}$ \\
\hline
\end{tabular}

\section{Validazione}

Una volta che ha avuto luogo il trasferimento, i file dovrebbero essere messi al sicuro, isolati, fatto un backup dell'ambiente operativo e avviata prontamente una verifica nel processo. Avendo trasferito e ospitato una copia di una collezione nativa digitale, un'organizzazione 
risponde quindi di certe responsabilità legali come Freedom of Information Requests se ci si trova nel settore pubblico. A seguito di ciò, dovrebbe essere inviata a colui che ha effettuato la donazione una lettera di comunicazione della ricezione. In una fase iniziale è importante che non vengano date istruzioni per la distruzione delle copie originali.

Alla prima occasione possibile si dovrebbe verificare la presenza di virus nei record (per assicurarsi che le risorse non siano state infettate da malware o virus). Se si è riscontrata qualche anomalia, l'ente depositario dovrebbe essere avvisato e i media dovrebbero essergli restituiti (se non ne hanno una copia) o formattati e restituiti o distrutti secondo le preferenze del depositante. Una volta che nei record non vengono riscontrati virus, si dovrebbe avviare una verifica per assicurarsi che risultino presenti tutti i record e che non siano danneggiati. II metodo più affidabile per farlo è verificare i file rispetto al manifest.

Si suggerisce la creazione dei checksum per i file e la comparazione con quelli elencati nel manifest prima del trasferimento. Se i checksum combaciano si può essere sicuri che i record non sono stati danneggiati o involontariamente alterati durante il trasferimento dal depositante fino all'arrivo nell'organizzazione, potrebbe essere impossibile verificare complessivamente l'integrità dei file e una visione manuale di un campione di file potrebbe essere necessaria per dare qualche indicazione di completezza e qualità. In questo caso, dovrebbe essere generato un manifest verificabile per permettere un controllo successivo della fixity.

Si spera che in questa fase i record saranno designati come completi (in linea con il manifest), conservando la loro integrità (esattamente ciò che il depositante ha offerto) e che non presenteranno virus o malware. Potranno quindi essere acquisiti nel sistema di conservazione digitale.

I metadati che descrivono le risorse depositate saranno di supporto per assicurare la fixity (si veda Fixity e checksum) delle risorse durante il processo di trasferimento così come della conseguente conservazione e accesso. Ciò potrebbe comprendere:

Un manifest verificabile che consiste in un elenco di file e nomi di cartelle e valori di checksum/fixity per ogni file

La dimensione dei file (con il volume totale)

Un elenco di formati dei file

Una dichiarazione che fornisce dettagli su qualsiasi IPR associato ai record

Ove possibile, l'onere a fornire delle informazioni sull'IPR dei record (dovrebbe rimanere al depositante).

\section{Processo di Ingest}

II periodo che intercorre tra il trasferimento verso l'ente e il processo di ingest nel repository dell'ente o nel campo della conservazione digitale può essere molto importante. Questa fase di selezione può essere prolungata nello specifico per le collezioni native digitali, a volte si tratta di anni, ma è durante questa fase che può essere avviata una valutazione qualitativa degli 
oggetti. I componenti vengono esaminati, poi vengono raccolti i loro metadati tecnici, e quelli descrittivi vengono migliorati, i processi generali di acquisizione dell'organizzazione sono applicabili alla sostituzione di qualsiasi oggetto.

È durante questo periodo di valutazione, a volte dilatato, che gli elementi vengono considerati per l'ingest, o rifiutati se nell'analisi non sembrano essere adatti per l'acquisizione o per il profilo della collezione dell'organizzazione, per la specificazione del formato dei file stabilita nei documenti di supporto, o per qualsiasi altra ragione. Potrebbe venire imposta una moratoria su dati particolarmente sensibili, come ad esempio le informazioni personali, informazioni commerciali sensibili, o dati che infrangono le leggi sulla diffamazione. In questi casi, è importante determinare chiaramente il periodo di chiusura dei file.

Pratiche di ingest per preparare i dati e la documentazione all'archiviazione e alla conservazione

Numerazione univoca

A ogni risorsa digitale acquisita da un ente dovrebbe essere assegnato un identificatore univoco. Questo numero identificherà la risorsa nel catalogo dell'Ente e sarà usato per riferirsi o individuare i supporti fisici e la documentazione. Se per una ragione o per un'altra una risorsa è stata disassegnata, questo numero univoco non dovrebbe essere riattribuito. Si veda Identificatori persistenti per dei suggerimenti se si utilizza lo schema di identificatore persistente.

Linee guida sulla gestione e sul trasferimento

Le linee guida sulla gestione e sul trasferimento rivolte al responsabile della selezione dovrebbero essere sviluppate sul modello IT e seguendo le raccomandazioni dei responsabili di conservazione sulle buone pratiche relative a diversi supporti di archiviazione e trasferimento di file su sistemi di archiviazione a lungo termine (si veda Supporti obsoleti, Informatica forense, e Archiviazione)

Riformattazione dei formati dei file

Laddove i formati dei file utilizzati per spostare le risorse non siano adatti alla conservazione nel lungo periodo, l'ente potrebbe riformattare le risorse nei formati prescelti dei file. Oltre ai formati d'archivio, anche le versioni in altri formati adatti ad essere presentati agli utenti possono essere generate a partire dall'originale (si veda Formati dei file e standard, e Archiviazione).

\section{Copia}

Diverse copie di backup di un oggetto potrebbero essere generate durante la selezione come parte dell'archiviazione dell'ente e di policy di conservazione, e per consentire le pratiche di ripristino di emergenza (si veda Archiviazione).

\section{Sicurezza}

Le strategie di sicurezza fisica e dei sistemi dovrebbero essere messe in atto per consentire il trattamento e l'integrità degli oggetti durante la selezione. Questi dovrebbero essere generati dalle politiche e pratiche istituzionali sulla sicurezza e poi rispecchiarle (si veda Sicurezza delle informazioni).

Modifica e controllo della tiratura 
Le modalità di aggiornamento e controllo del numero di copie di qualsiasi risorsa digitale dinamica aggiunta (ad es., istantanee annuali dei database che si stanno regolarmente aggiornando) o il controllo della versione degli oggetti aggiunti ove opportuno (ad es., gli oggetti aggiunti sotto diversi formati o per i quali sono stati generati diversi formati per la conservazione e l'accesso).

\section{Catalogazione e standard di documentazione}

I metadati e la documentazione ricevuta o creata durante il trasferimento, la validazione e l'ingest sono fondamentali per scambiare in modo efficace le informazioni e i documenti tra le piattaforme e i singoli individui. Quanto meno si dovrebbero fornire le informazioni riguardo alla provenienza degli oggetti e all'iter amministrativo (includendo ogni elaborazione di dati coinvolti sin dalla loro realizzazione), al contenuto, alla struttura e ai termini e condizioni allegati, alla loro conseguente gestione e utilizzo, inclusi i diritti IPR e al periodo a cui si riferiscono (si veda Metadati e documentazione). Dovrebbero essere abbastanza dettagliati per supportare:

- La ricerca delle risorse (ad es., il posizionamento di una risorsa che è stata almeno brevemente descritta insieme a molte altre risorse).

- La valutazione delle risorse (ad es., il processo con il quale un utente determina se si ha necessità di accedere a quella risorsa).

- L'ordine delle risorse (ad es., quelle informazioni che istruiscono l'utente sui termini e sulle condizioni allegate alla risorsa e sui processi o altri mezzi con cui l'accesso a una determinata risorsa potrebbe essere ottenuto).

- L'utilizzo delle risorse (ad es., l'informazione amministrativa essenziale alla gestione e conservazione delle risorse come parte di una collezione più ampia, includendo informazioni come la posizione, il controllo della versione, ecc).

Tempi di elaborazione

Idealmente, i traguardi dovrebbero essere impostati e monitorati per il tempo massimo che intercorre tra l'acquisizione e la catalogazione al fine di evitare che ci siano arretrati di risorse che si generano durante il processo di selezione, risorse non processate e potenzialmente a rischio.

\section{Competenze, risorse e capacità}

Le organizzazioni dovrebbero tenere in considerazione il fatto di disporre o meno di sufficienti risorse tecniche e di personale per acquisire le collezioni digitali. Tuttavia, queste informazioni potrebbero non essere evidenti all'inizio del processo di acquisizione, poiché è soltanto con il tempo che potrebbero emergere diversi problemi relativi alla conservazione di specifiche collezioni digitali. Perciò, le organizzazioni dovrebbero definire un piano per lo sviluppo della conoscenza, competenza e colmare i divari esistenti tra il personale, e, ove possibile, occuparsi di questi ultimi mediante formazioni, assunzioni o attraverso servizi di conservazione digitale professionali.

Laddove non bastassero le risorse, spesso una buona soluzione potrebbe essere quella di offrire dei percorsi all'interno dell'organizzazione di sviluppo delle competenze (si veda Formazione del personale e sviluppo e Acquisizione e servizi di parti terze).

\section{Costi di acquisizione e ingest}

È sempre problematico stabilire dei costi indicativi per le attività di conservazione digitale. Questi ultimi non dovrebbero solo includere l'archiviazione (il più ovvio), ma dovrebbero anche riguardare il costo del tempo del personale necessario per gestire la selezione e l'ingest per 
ognuno degli oggetti nativi digitali, un processo speculare al percorso di acquisizione degli artefatti fisici. Altri costi previsti potrebbero comprendere i processi di curation come la normalizzazione, l'analisi dei metadati, una maggiore solidità dell'archivio, un ripristino di emergenza, ecc.

Nonostante un'organizzazione debba trovare delle soluzioni più valide relative ai costi associati al ciclo di vita, bisogna riconoscere che l'investimento richiesto per garantire un solido percorso di conservazione, che può salvaguardare il nostro patrimonio digitale, può essere importante $\mathrm{e}$ che certi processi devono essere rispettati a prescindere dalla natura dell'oggetto acquisito. Tutto ciò dovrebbe essere tenuto in considerazione nel momento di acquisizione di collezioni native digitali. (si veda $\underline{\text { Casi aziendali, benefici, costi e impatto) }}$

\section{Sintesi delle raccomandazioni}

Acquisizione e valutazione - raccomandazioni, liste di controllo

Accordi e Istruzioni per i detentori

$\square$ Creare una suite di accordi e licenze standard per i detentori

$\square$ Creare istruzioni appropriate per i detentori

\section{Procedure di trasferimento}

$\square$ Fornire una documentazione per guidare e supportare il trasferimento di risorse digitali dai fornitori

$\square$ Stabilire quanto le proprie procedure di trasferimento possano essere sviluppate per sostenere al meglio le proprie politiche di archiviazione e conservazione

\section{Procedure di validazione}

$\square$ Verifica dei media, del contenuto e della struttura

Procedure per preparare i dati e la documentazione all'archiviazione e alla conservazione

$\square$ Numerazione univoca per ogni oggetto integrato

$\square$ Linee guida sulla gestione e trasferimento dell'archiviazione su diversi media

$\square$ Riformattazione dei formati dei file se richiesto in maniera conforme alle linee guida concordate

$\square$ Generazione di diverse copie di un oggetto come parte dell'archiviazione dell'ente e della politica di conservazione

$\square$ Politica di sicurezza fisica e dei sistemi e procedure relative agli oggetti durante la l'acquisizione.

\section{Procedure per la catalogazione e documentazione}

- Uno standard minimo delle informazioni necessarie per la catalogazione, incluse le informazioni IPR

$\square$ Linee guida per la documentazione retrospettiva o potenziamento del catalogo

- Procedure per l'aggiornamento e gestione delle versioni o tirature di un oggetto

- Procedure per l'aggiornamento dei database di gestione della collezione

$\square$ Selezione di standard di catalogazione e documentazione

$\square$ Obiettivi per le attività di selezione e tempistiche per il loro completamento 
Analisi delle procedure

- La valutazione delle linee guida e dei programmi dovrebbe avvenire idealmente ogni anno o tutte le volte che sarà necessario per stare al passo delle necessità di sviluppo di un'organizzazione e con le politiche di sviluppo delle collezioni

Formazione del personale

- Pianificazione delle conoscenze, competenze e divari nel personale e, ove possibile, occuparsi di questi ultimi mediante formazioni, assunzioni o impiego di servizi specialistici di parti terze

Costi

$\square$ Valutare e pianificare i costi del ciclo di vita delle acquisizioni.

\section{Risorse}

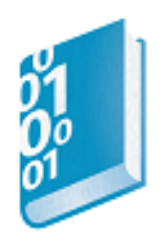

ISO 20104:2015 Space data and information transfer systems -- Producer-Archive Interface Specification (PAIS)

CCSDS 651.1-B-1, Producer-Archive Interface Specification (PAIS)

(2014)

RECOMMENDED STANDARD CCSDS 651.1-B-1 BLUE BOOK February 2014

https://public.ccsds.org/Pubs/651x1b1.pdf

II Blue Book è una prestampa a libero accesso dello standard ISO 20104:2015. Lo standard PAIS ha lo scopo di fornire una metodologia standard per la definizione formale degli oggetti digitali da trasferire attraverso un Produttore informatico a un Archivio e per la messa a punto efficace di questi oggetti sotto forma di Pacchetti di versamento Submission Information Packages (SIPS). Ciò supporta il trasferimento e la verifica effettiva dei dati SIP (104 pagine).

\section{What is appraisal?}

http://www.nationalarchives.gov.uk/documents/information-management/what-is-appraisal.pdf

Questa guida del National Archives si applica ai record pubblici britannici in qualsiasi formato, inclusi gli articoli, formati digitali, audio, film o modelli di formati così come definito dal Public Records Act del 1958, e a tutte le organizzazioni responsabili di tali record (2013, 7 pagine).

\section{Preserving eBooks, DPC Technology Watch Report 14-01 July 2014}

http://dx.doi.org/10.7207/twr14-01

Questo report analizza gli attuali sviluppi e le questioni che le biblioteche pubbliche, nazionali, e di istruzione superiore, gli editori, gli aggregatori, e gli istituti di conservazione devono affrontare per assicurare l'accesso a lungo termine al contenuto eBook, e che influenzano l'acquisizione così come la conservazione (31 pagine). 
Preservation, Trust and Continuing Access for e-Journals, DPC Technology Watch Report 13-04 September 2013

http://dx.doi.org/10.7207/twr13-04

Questo report analizza gli attuali sviluppi e le questioni che le biblioteche, gli editori, gli intermediari e i fornitori di servizi stanno affrontando nell'ambito della conservazione digitale, della fiducia e dell'accesso continuo alle riviste elettroniche. Non si concentra solamente sulla tecnologia, ma si interessa anche degli aspetti legali, economici e di servizio rilevanti nell'ottenimento dell'accesso a risorse digitali interconnesse, nonché le sfide uniche di conservazione. (43 pagine).

The UNESCO/PERSIST Guidelines for the selection of digital heritage for longterm preservation

https://unesdoc.unesco.org/ark:/48223/pf0000244280

II progetto UNESCO/PERSIST (Platform to Enhance the Sustainability of the Information Society Transglobally) ha pubblicato queste linee guida sulla selezione del patrimonio documentario per la conservazione a lungo termine nel marzo 2016. Lo scopo delle linee guida è quello di garantire un punto di partenza generale per le biblioteche, gli archivi, i musei e altri istituti di conservazione del patrimonio nel momento in cui redigono le proprie politiche sulla selezione del patrimonio documentario per la conservazione digitale sostenibile a lungo termine. (19 pagine).

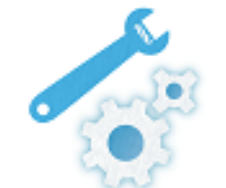

Community Owned digital Preservation Tools Registry COPTR

http://coptr.digipres.org/Main Page

COPTR descrive gli strumenti che si prestano per la conservazione digitale a lungo termine e si può considerare principalmente come uno strumento di individuazione e valutazione che aiuta $i$ professionisti a ricercare gli strumenti di cui hanno bisogno per conservare i dati digitali. COPTR identifica i dettagli di base, concreti di uno strumento, scopre cosa fa lo strumento e la modalità per individuare maggiori informazioni (URL rilevanti) e riferimenti alle esperienze degli utenti con gli strumenti. L'ambito fa riferimento all'accezione più generale del termine 'conservazione digitale'. In altre parole, se uno strumento è utile nell'esecuzione delle funzioni della conservazione digitale, come quelle descritte nel modello OAIS o nel modello del ciclo di vita DCC, allora rientra nell'ambito di questo registry. Ė possibile utilizzare la POWRR Tools Grid per vedere quali strumenti in COPTR possono supportare l'acquisizione, l'ingest o altre funzioni.

\section{Keepers Registry}

https://keepers.issn.org/

II Keepers Registry fa da monitoraggio globale alle disposizioni archivistiche per le riviste elettroniche. Comprende tre obiettivi principali: permettere ai bibliotecari e ai decisori politici di identificare chi è che sta cercando una determinata rivista elettronica, come e con quali termini d'accesso; mettere in risalto quali sono le riviste elettroniche ancora 'a rischio di essere perdute'; 
presentare le organizzazioni (custodi) che fungono da scaffali digitali per l'accesso sul lungo periodo. Con la caratteristica Title List Comparison si può scoprire lo status dell'archivio relativo a una lista di titoli seriali d'interesse: si riportano quelli che si stanno archiviando e quelli 'a rischio'.

MediaRIVERS (Media Research and Instructional Value Evaluation and Ranking System) https://github.com/IUMDPI/MediaSCORE

Un Software creato dall'Indiana University in collaborazione con AVPreserve, guida a una valutazione strutturata del valore di ricerca ed educativo per le collezioni su supporti. La versione gratuita open source prevede l'installazione e la configurazione su un server, mentre l'applicazione hosted è disponibile con iscrizione mensile.

\section{Practical E-Records: software and tools for archivists http://e-records.chrisprom.com/}

Le pagine create da Chris Prom per Transfer Guidelines, E-Records Deposit Policy, and Submission Agreement Form forniscono dei modelli di template che si possono modificare e/o dare ai produttori di record che il proprio repository desidererebbe acquisire. La licenza di modifica e di ripubblicazione di queste linee guida di trasferimento è rilasciata sotto la Creative Commons Attribution 3.0 United States License.

WWW

\section{Archaeology Data Service Guidelines for Depositors}

http://archaeologydataservice.ac.uk/advice/guidelinesForDepositors

Le linee guida ADS per i detentori offrono una guida su come preparare in modo corretto i dati e compilare i metadati per depositare questi ultimi con ADS, e descrivono le modalità in cui $i$ dati possono essere depositati. Vi sono inoltre una serie di schede riepilogative più brevi e liste di controllo che riguardano: la gestione dei dati; la selezione e il mantenimento; i formati dei file preferiti e i metadati. Altre risorse per l'uso di detentori potenziali comprendono una serie di Guides to Good Practice, che si integrano con le linee guida ADS e forniscono un tipo di informazione più dettagliata sui tipi di dati specifici.

\section{Selecting and transferring records}

http://www.nationalarchives.gov.uk/information-management/manage-information/selectionand-transfer/

Queste pagine presentano una guida sulla selezione e sul trasferimento dei record. Gli istituti britannici che trasferiscono i record al National Archives o a spazi di depositi sulla base del Public Records Act del 1958 dovrebbero seguire questo processo per i record in tutti i formati e supporti, compresi i record su carta e digitali. Comprende sei passaggi:

Step 1: Valutazione dei propri record

Step 2: Selezione dei propri record

Step 3: Revisioni dei record selezionati per identificare i dati sensibili

Step 4: Catalogazione e preparazione dei record 
Step 5: Pianificazione e modalità di presentazione dei record Step 6: Selezione dei propri record

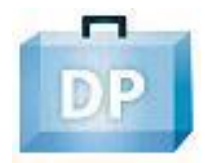

The Work of Appraisal in the Age of Digital Reproduction

http://archival-integration.blogspot.co.uk/2015/06/the-work-of-appraisal-in-age-ofdigital.htm|\#pii

II progetto Bentley Historical Library's ArchivesSpace-Archivematica-DSpace Workflow Integration mette in evidenza le tecniche attuali di valutazione degli archivi digitali utilizzate dalla Bentley, molte delle quali si spera saranno integrate all'interno di Archivematica (Giugno 2015).

\section{Acquisition \& management of digital collections at the Library of Congress}

http://www.slideshare.net/NASIG/acquisition-management-of-digital-collections-at-the-libraryof-congress-34244613

La Library of Congress, essendo una biblioteca nazionale e punto di riferimento della US Copyright Office, è pienamente coinvolta nell'acquisizione e gestione digitale. Questo breve e informativo powerpoint di Ted Westervelt presenta le esperienze della Library of Congress e le lezioni apprese. (2014, 30 slide).

\section{Trust Me, I'm an Archivist: Experiences with Digital Donors}

http://www.ariadne.ac.uk/issue65/hilton-et-al

Questo articolo del 2010 dello staff della Wellcome Trust Library presenta quattro scenari che sembrano rappresentare nuovi limiti per il trasferimento delle risorse digitali: Lack of Long-term Planning; IT vs Records Management; Duplication and Abundance; and The Fear of Digital. Si conclude con una considerazione relativa al fatto che ciò che bisogna cambiare è il modo in cui si presenta l'informazione, in cui si lavora con le risorse digitali e in cui si possono supportare e assistere i donatori. II livello di impegno, pratica comune nella gestione di record su stampa, non è abbastanza per risorse native digitali: l'interazione con i detentori sarà idealmente ancora più stretta e più frequente, dal momento che questi ultimi verranno supportati non solo nelle sfide tecniche, ma anche in una serie di questioni riferite a competenze trasversali, a preconcetti e a una serie di legami esistenti. 


\section{Alberi decisionali}

La chiara definizione di strategie selettive consentirà risparmi economici in termini di tempo dedicato a stabilire se si debba o meno avviare un processo di selezione e anche di costi potenziali che si sosterranno per l'eventuale rivalutazione delle risorse digitali che rischiano sia di diventare inaccessibili o che già lo sono.

Questi Alberi decisionali possono essere utilizzati come strumenti per realizzare o testare una policy del genere per la propria organizzazione. II processo decisionale rappresentato negli alberi dovrebbe essere oggetto della propria strategia di selezione delle risorse digitali sul lungo periodo.

Partendo dal presupposto che le risorse digitali vengono prese in considerazione per la fase di selezione, le questioni e le scelte qui discusse supporteranno la decisione finale di accettare o rifiutare la responsabilità della conservazione nel lungo periodo. II flusso delle domande rappresenta un ordine logico di valutazione. Se la risposta alle prime domande non è favorevole ha poco senso accettare la responsabilità della conservazione per la risorsa o continuare la sua valutazione, ad esempio se il contenuto non è adatto alla politica della collezione, allora la risposta alle domande sul formato tecnico sarà irrilevante. La struttura degli alberi mira a rispecchiare questo processo.

Si tenga presente che...

Quando viene messa in atto una policy, perché questa sia effettiva deve anche essere:

- Approvata dai dirigenti

- Emanata attivamente per tutta l'organizzazione

- Analizzata ad intervalli regolari

- Destinataria di risorse appropriate

Si vada alla sezione Valutazione interattiva degli alberi decisionali.

La versione interattiva richiede che venga abilitato sul proprio computer javascript.

Valutazione interattiva

Valutazione interattiva: Selezione delle risorse digitali per la conservazione a lungo termine 


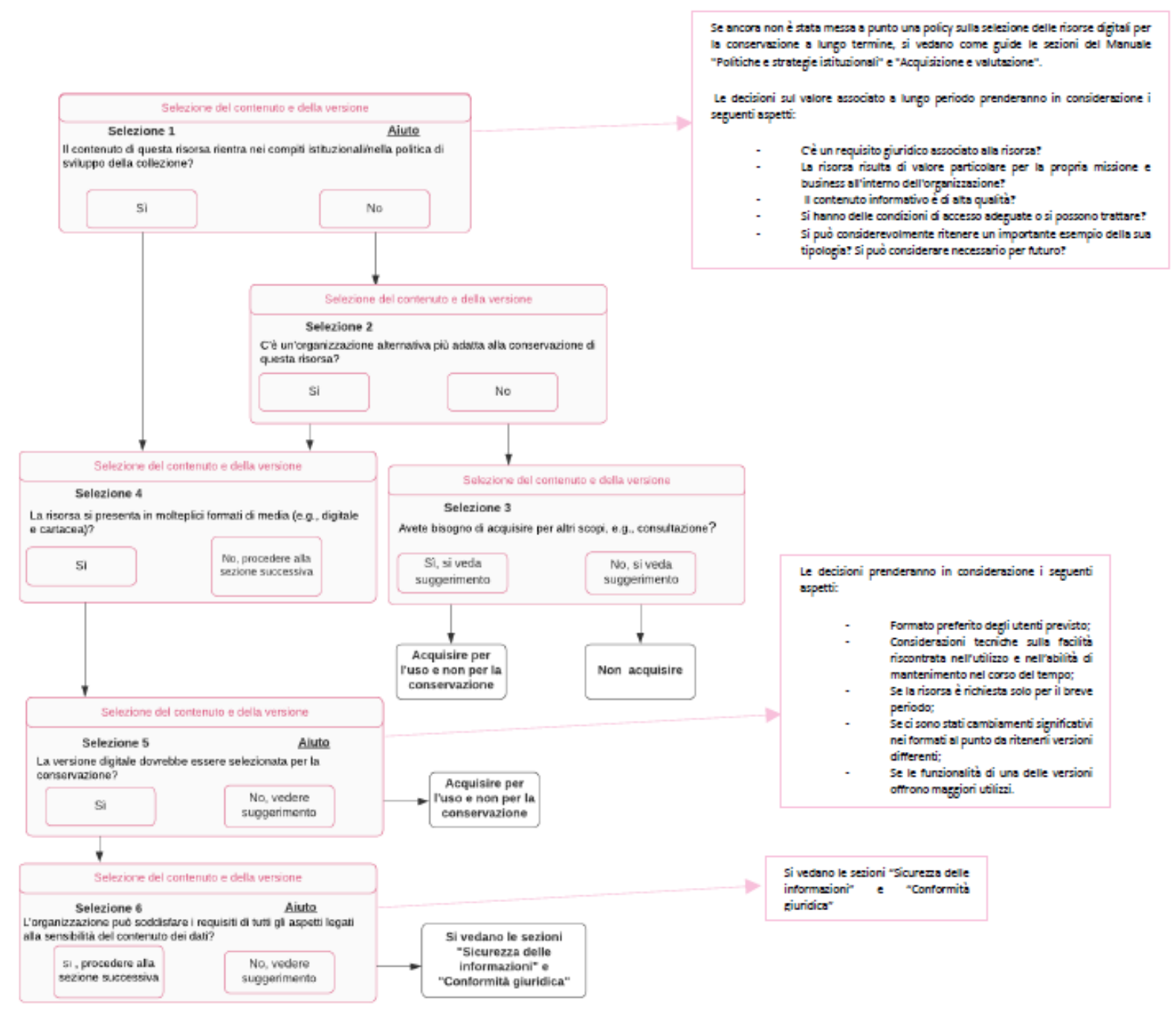




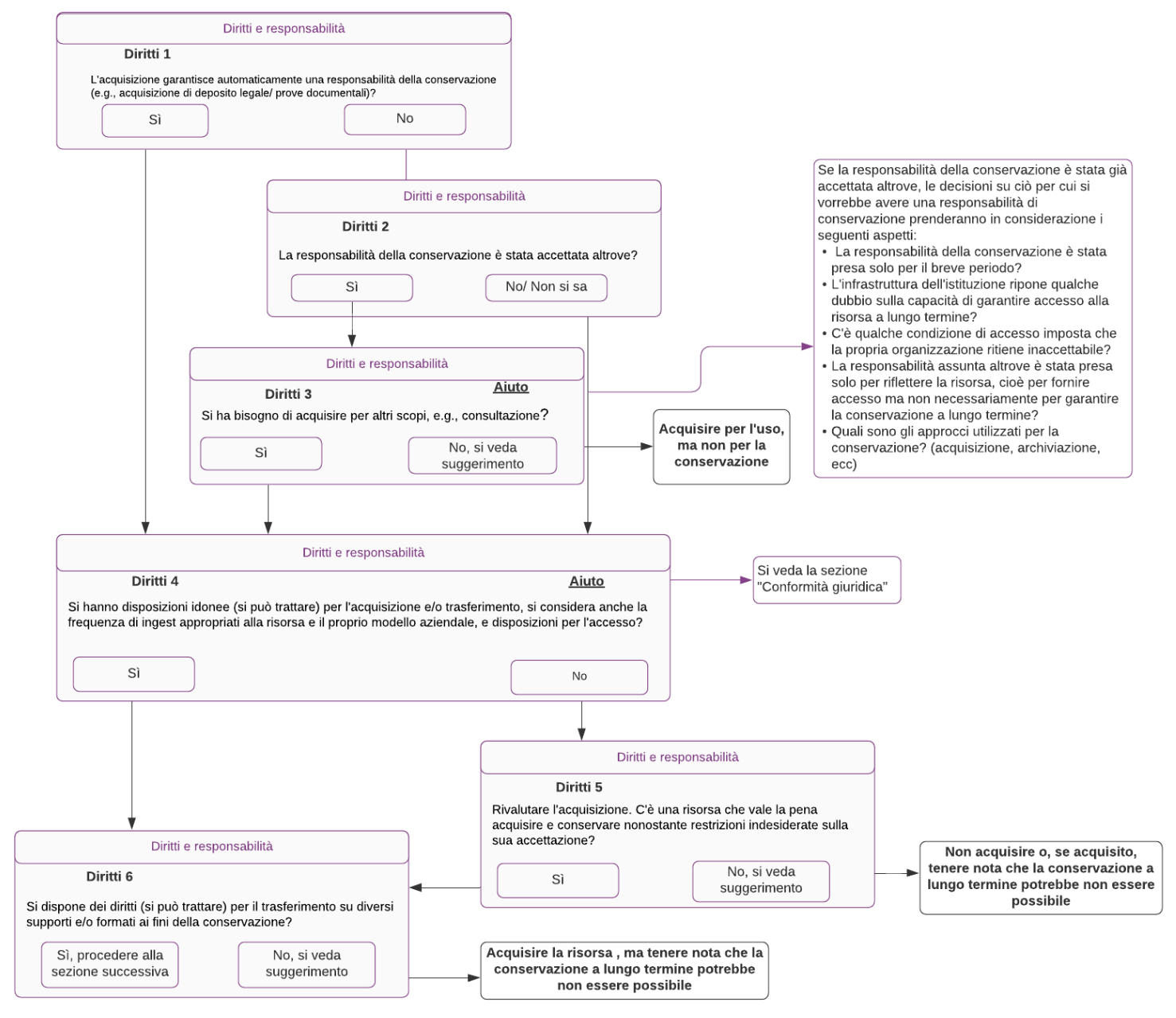




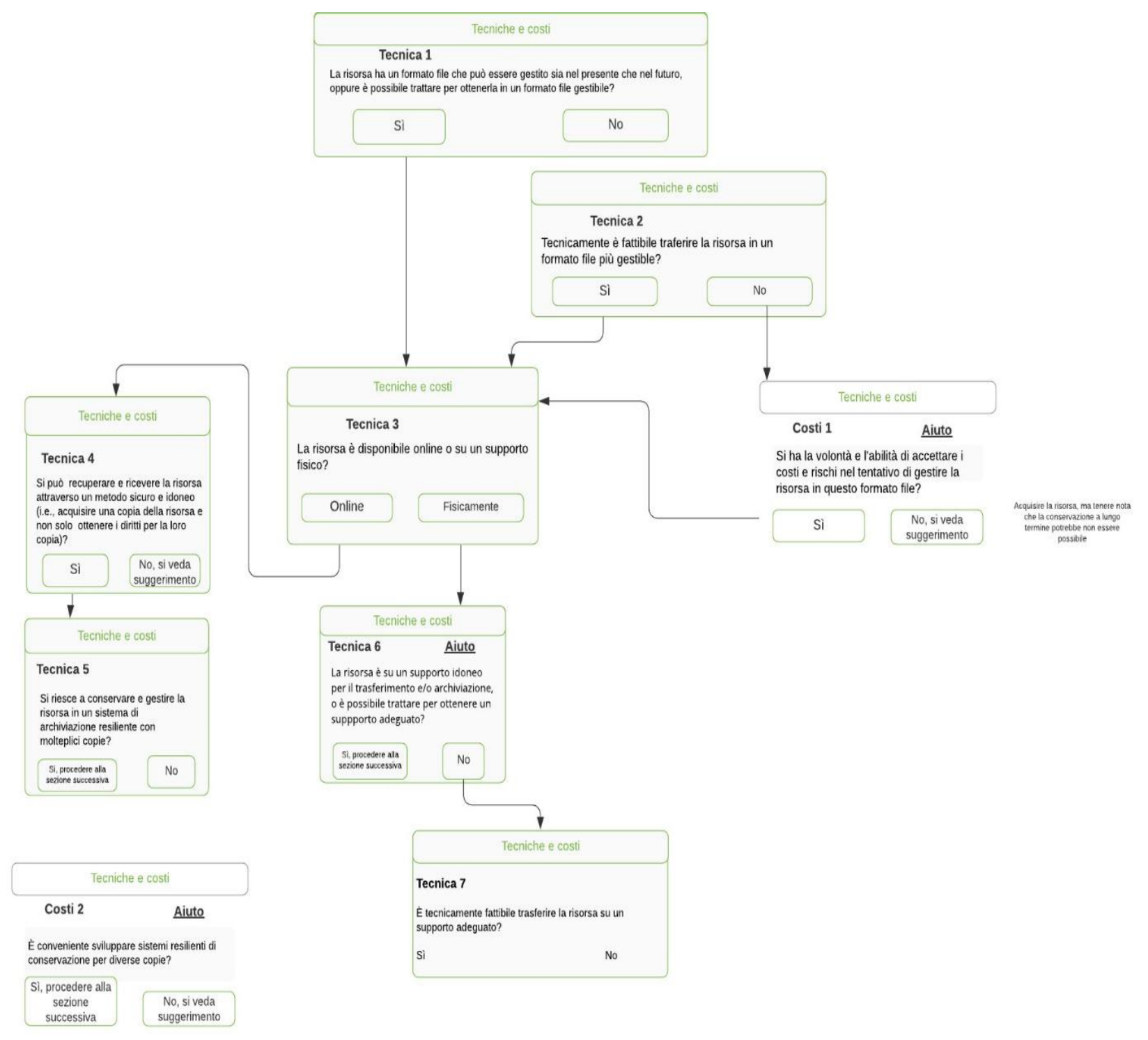




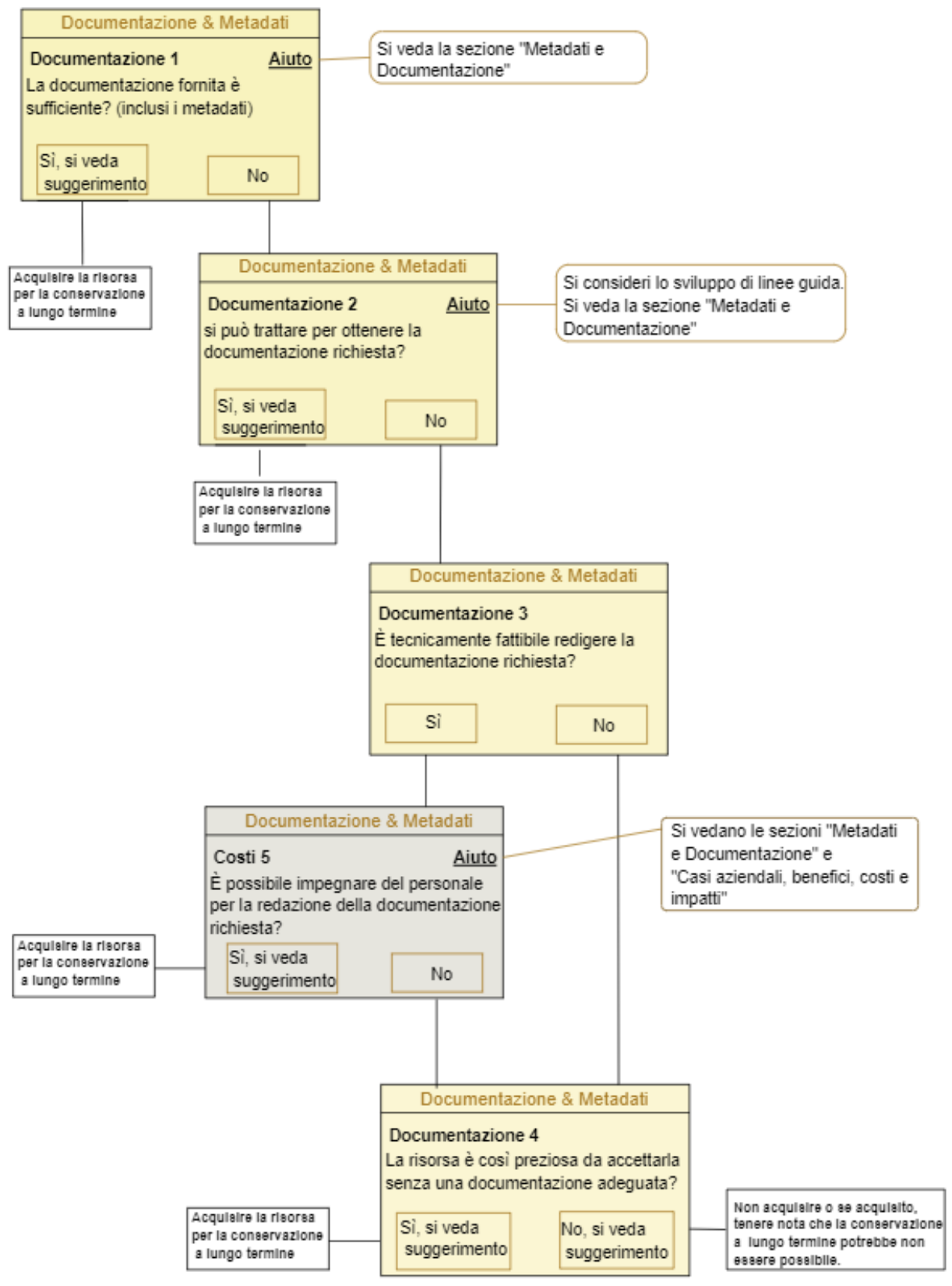




\section{Conservazione e revisione}

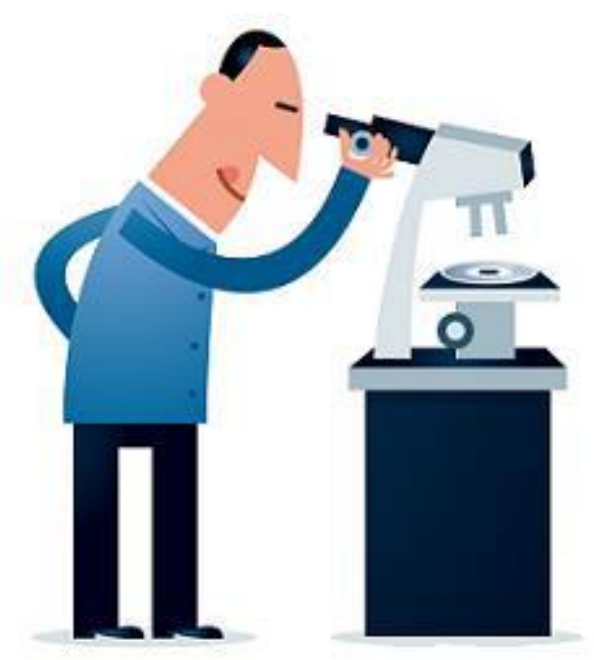

Illustrazione di Jørgen Stamp digitalbevaring.dk CC BY 2.5 Denmark

La scelta della conservazione a lungo termine generalmente si verifica nella fase di acquisizione, ma può essere un processo iterativo che si presenta in fasi successive una volta che un oggetto si trova già all'interno delle collezioni. I termini conservazione e revisione vengono utilizzati in questo Manuale per il processo iterativo. II processo decisionale rispecchia i passaggi inclusi nelle sezioni Alberi decisionali e Acquisizione e valutazione e gli alberi possono essere adattati a questo scopo.

Adottare dei criteri di valutazione e pratiche di selezione per tutte le possibili acquisizioni digitali assicura che venga attentamente data priorità allo sviluppo delle collezioni e che questo sia reso sostenibile. Con questi criteri e procedure si dovrebbero ridurre la frequenza e il bisogno della conservazione e di decisioni valutative, poiché le acquisizioni vengono accuratamente valutate e giustificate prima che queste entrino nelle collezioni. Le organizzazioni potrebbero anche avere bisogno di conservare certi record interni e risorse digitali per necessità normative, legali, operative e finanziarie. Queste dovrebbero essere gestite altrettanto attivamente per preservare la fattibilità, l'autenticità e l'accessibilità.

Gli oggetti digitali acquisiti nel tempo, e prima dell'implementazione di politiche e procedure istituzionali, generalmente comporteranno una fase di valutazione. Quest'ultima può essere effettuata nei primi passaggi che intraprende un ente nell'implementazione della politica di conservazione digitale: quantificazione della sua collezione digitale attuale e valutazione dei rischi di conservazione (si veda Guida introduttiva).

Gli archivi utilizzano il concetto di serie per il contenuto dei record che condividono caratteristiche simili. Generalmente, molte serie durano diversi decenni. Tuttavia, la portata e la copertura di una serie digitale potrebbero variare nel tempo e sicuramente potrebbero cambiare 
delle considerazioni sulla tecnologia, e sarebbe opportuno fare un'accurata valutazione dal momento che ogni acquisizione viene trasferita agli archivi.

Nel corso del tempo ci potrebbe essere necessità di revisionare le collezioni e che le politiche delle collezioni riflettano le necessità e le circostanze cambiate. La necessità di prendere decisioni preliminari sulla selezione della conservazione nell'ambito digitale (senza possibilità di agire a posteriori, cosa che spesso avviene in ambito analogico) potrebbe comportare l'esigenza di una futura revisione nel ciclo di vita della conservazione delle risorse digitali.

Nelle biblioteche digitali in cui vengono usati i livelli delle collezioni, le risorse digitali ad ogni categoria di livello della collezione possono essere soggette a una revisione periodica, riassegnate da un livello a un altro, ritirate, o dis-integrate se richiesto per aderire ai requisiti e alle circostanze che sono cambiate. Tuttavia, per gli oggetti selezionati per la conservazione permanente viene previsto che la revisione e la selezione si verifichino in circostanze rare e strettamente monitorate. Per altri livelli delle collezioni, come le risorse speculari o autorizzate, i criteri di revisione possono comprendere:

- Un crollo costante di utilizzo al di sotto di un livello accettabile.

- La disponibilità del contenuto da altre parti a un livello più alto di qualità o a un costo considerevolmente basso.

Qualora il contenuto venga sostituito o non risulti sufficientemente accurato per giustificarne il mantenimento in forma attiva, potrebbe essere conservato insieme al numero di edizioni successive oppure venire ritirato.

- Scadenza o termine di una licenza o accordo di scambio di dati e ritiro/restituzione della risorsa digitale al fornitore di dati.

- Il costo per sostenere la risorsa di dati supera il valore/beneficio ricevuto.

- Deterioramento nella qualità del servizio fornito da un fornitore o nell'accessibilità del contenuto a causa di uno scarso aggiornamento dell'indicizzazione, imaging, o altre caratteristiche interne alla risorsa di dati.

Disporre di un piano di conservazione è prassi ben consolidata nelle professioni relative alla gestione documentale e all'archiviazione. I record potrebbero venire distrutti alla fine del loro periodo di conservazione, conservati per un ulteriore lasso di tempo o spostati presso un ente per la conservazione a lungo termine.

In qualsiasi ambiente relativo alle collezioni, è importante che le procedure scritte siano messe in atto per il processo di conservazione e revisione con chiare responsabilità attribuite a persone specifiche o a quelle sezioni di un'organizzazione responsabili dell'amministrazione e sviluppo delle collezioni. Gli intervalli di tempo, le circostanze e le pratiche di autorizzazione per la revisione dovrebbero essere chiaramente definite. I documenti di pianificazione della conservazione e revisione dovrebbero essere essi stessi revisionati periodicamente per stare al 
passo delle necessità emergenti di un'organizzazione. A seconda dell'ambiente aziendale, i rispettivi utenti e detentori potrebbero essere consultati come parte del processo. Qualsiasi loro suggerimento può essere sottoposto a verifica da parte dell'amministrazione e dei comitati se in linea con la dimensione e valore della risorsa.

Quando si indicano delle linee guida sulla selezione di una risorsa archiviata, dovrebbero esserci delle procedure da discutere con altri stakeholder al fine di decidere se deve avvenire il trasferimento a un'altra organizzazione. In tali casi, l'ente dovrebbe concordare delle condizioni di trasferimento che comprendano livelli accettabili di trattamento delle risorse e accesso in modo adeguato per gli utenti appartenenti al mondo dell'istruzione e della ricerca. Le limitazioni economiche non dovrebbero rappresentare un motore principale per la selezione degli oggetti digitali poiché il processo in sé comporta dei costi e potrebbe far emergere delle questioni etiche spinose. Le decisioni riguardo alla selezione dovrebbero essere guidate principalmente dalla politica di sviluppo delle collezioni.

Le risorse digitali acquisite, che non sono state conservate dopo la revisione, dovrebbero preservare il loro ingresso in qualsiasi catalogo istituzionale con dei commenti che identifichino il processo intrapreso e ogni dettaglio sul trasferimento.

Anche la pianificazione della successione da parte di un ente potrebbe essere rilevante per la conservazione e revisione. Lo standard per Audit and Certification of Trustworthy Digital Repositories (CCSDS, 2011) recommended practice 3.1.2.1 dispone che il repository dovrà avere un piano di successione adeguato, piani di contingenza e/o accordi fiduciari messi in atto se il repository cessasse di funzionare o se l'ente direttivo o finanziario cambiasse in modo sostanziale il suo campo d'applicazione.

\section{Risorse}

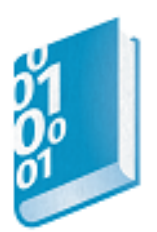

Deaccessioning and disposal: Guidance for archive services

http://www.nationalarchives.gov.uk/documents/Deaccessioning-and-disposal-guide.pdf

Questa guida è stata scritta al fine di supportare l'Archive Service Accreditation, lo standard generale britannico per i servizi di archivi. È una guida generale e si può applicare al contenuto in qualsiasi formato esso si presenti (analogico o digitale) e non comprende disposizioni speciali per le risorse digitali. Lo standard presenta l'attività di selezione come parte dello sviluppo delle collezioni e prevede che i servizi di archivio dispongano di politiche, piani e procedure da mettere in atto per le attività di sviluppo delle collezioni, inclusa la selezione. Comprende anche un albero decisionale relativo alla disposizione finale. (2015, 34 pagine). 


\section{Digital Preservation Management Tools: Digital Content Review process}

http://dpworkshop.org/workshops/management-tools/process-results

Al fine di rendere completo il processo di revisione del contenuto digitale, il team della conservazione digitale ha riunito le informazioni e le ha iterativamente accumulate come parte di un processo strutturato. I risultati delle revisioni in corso del contenuto digitale hanno portato ad avere un dataset di revisione del contenuto digitale che ha permesso alle organizzazioni di avere un sistema di pianificazione a breve e lungo termine.

The National Archives, Disposing of records

http://www.nationalarchives.gov.uk/information-management/manage-information/policy-

process/disposal/

TNA ha realizzato una guida passo dopo passo per aiutare gli utenti nel processo di scarto compresa una lista di controllo dello scarto.

The British Library's de-accessioning policy

http://www.bl.uk/aboutus/stratpolprog/coldevpol/deaccessioning/

Questa policy definisce le circostanze per cui la British Library potrebbe scartare alcune tipologie di risorse. È una policy generica che si applica ai contenuti in qualsiasi formato essi si presentino (analogici o digitali) e non comprende disposizioni speciali per le risorse digitali.

\section{Riferimenti}

Consultative Committee for Space Data Systems (CCSDS), 2011. Audit and Certification of Trustworthy Digital Repositories Recommended Practices CCSDS 652.0-M-1 Magenta Book September 2011 [this was subsequently published as ISO 16363: 2012]. Disponibile al seguente link: https://public.ccsds.org/pubs/652x0m1.pdf 


\section{Archiviazione}

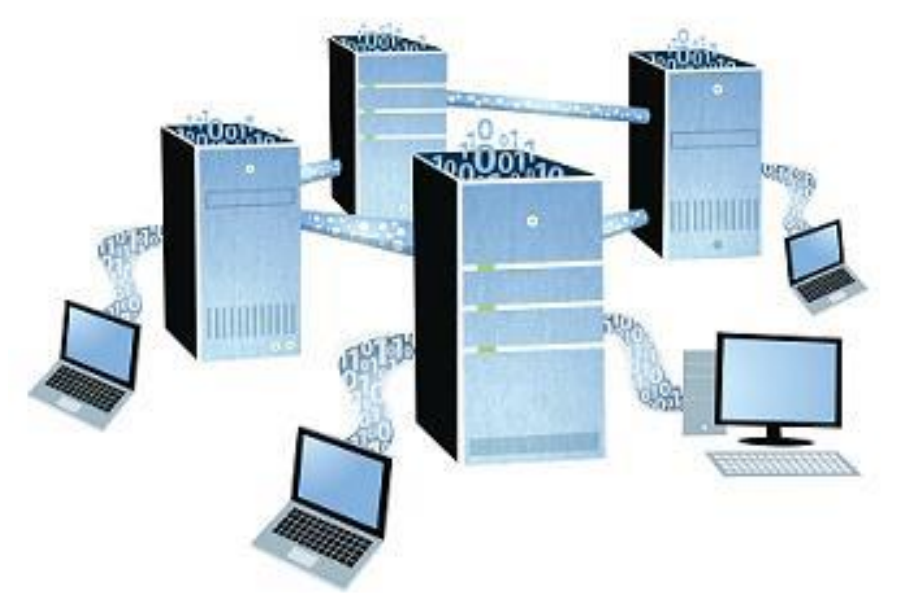

Illustrazione di Jørgen Stamp digitalbevaring.dk CC BY 2.5 Denmark

\section{Introduzione}

In questa sezione si descriverà la pratica emergente dell'uso di sistemi di archiviazione per la conservazione digitale. Si presenteranno gli aspetti generici, mentre quelli più specifici associati all'archiviazione cloud sono trattati separatamente (si veda la sezione Servizi Cloud). Anche l'attività tradizionale della conservazione di media digitali, per esempio gli oggetti obsoleti all'interno di collezioni esistenti, viene specificata più nel dettaglio all'interno della sezione Supporti obsoleti.

Diverse organizzazioni avranno combinato strategie o staranno per effettuare una transizione dall'una all'altra. L'uso della tecnologia di archiviazione per la conservazione digitale è drasticamente cambiato nel corso degli ultimi venti anni. Durante questo periodo di tempo, il cambiamento è avvenuto nella pratica. Precedentemente, la norma prevedeva l'archiviazione delle risorse digitali utilizzando dei supporti multimediali statici, ad esempio i CD, le cassette, ecc., periodicamente migrati per far fronte al degrado e obsolescenza. Attualmente, è più comune l'utilizzo di sistemi di archiviazione IT per ampi volumi di risorse digitali in crescita che necessitano di essere conservate, e forse soprattutto che hanno bisogno di essere facilmente e rapidamente recuperabili in un contesto di accessi online. In questo modo, le risorse digitali si sono disgiunte dal meccanismo sottostante la loro archiviazione. Da ciò ne conseguono benefici che consentono differenti attività di conservazione che devono essere gestite indipendentemente.

\section{Sistemi di archiviazione resilienti}

Un sistema di archiviazione resiliente IT è rappresentato da archivi multimediali contenuti all'interno di un server che garantisce una resistenza intrinseca alle varie forme di errori attraverso l'utilizzo di ridondanza integrata per il recupero. Ad esempio, un sistema di archiviazione potrebbe essere un dispositivo hard disk in un Redundant Array of Independent Disks (RAID), cassette di dati in una libreria a nastro, o una combinazione di tipi di archiviazione 
in un Hierarchical Storage Management system (HSM). Ciò può comprendere un archivio in loco e/o un cloud remoto e una replicazione automatizzata delle risorse digitali in diversi luoghi e sistemi.

Questi sistemi diventeranno comunque obsoleti nel tempo e le risorse digitali dovrebbero essere migrate tra i sistemi di archiviazione nel momento in cui divengono obsoleti. La migrazione tra i sistemi di archiviazione è separata da quella tra i formati dei file e può essere ampiamente gestita come una dinamica IT, con la condizione che venga effettuata una supervisione appropriata per fare in modo che le esigenze di conservazione vengano soddisfatte. II vantaggio è che l'uso di sistemi IT per l'archiviazione dei dati può fornire un accesso più rapido, una soluzione più scalabile, una gestione più facile, costi fondamentalmente più bassi specialmente su scala.

Diventa cruciale capire la differenza tra le soluzioni standard di archiviazione IT e i bisogni aggiuntivi della conservazione a lungo termine. È essenziale essere in grado di spiegare queste differenze al proprio reparto IT o fornitore del servizio di archiviazione e di specificare queste necessità nel momento in cui si offre un sistema o un servizio. I sistemi di archiviazione standard sono progettati per gli oggetti digitali in uso corrente. Mentre le pratiche di backup di solito sono incluse, normalmente non soddisfano i requisiti più rigidi per assicurare la conservazione delle risorse digitali nel lungo periodo. II backup e la conservazione digitale non rappresentano la stessa cosa, e questo non è congeniale per diverse strutture IT o esperti del settore. I sistemi di archiviazione e conservazione prevedono un livello più alto di ridondanza geografica, un ripristino di emergenza più forte, una pianificazione a lungo termine, e, soprattutto, un monitoraggio attivo dell'integrità dei dati per rilevare cambiamenti indesiderati come il danneggiamento dei file o la loro perdita.

Esistono diversi modi per adempiere ai requisiti di archiviazione di conservazione e questi varieranno in riferimento alla dimensione e alla complessità del contesto organizzativo. Sarà necessario valutare le risorse interne e tenere in conto opzioni come l'esternalizzazione e l'archiviazione cloud. L'approccio adottato dipenderà spesso dalla dimensione della collezione e dalla sua complessità e dalle risorse che sono disponibili all'interno dell'organizzazione. È possibile soddisfare i requisiti di archiviazione di conservazione con impostazioni di base, ma dal momento che le collezioni aumentano in dimensioni, si dovrà trattare di aspetti come la scalabilità e l'automatizzazione. 
Principi per l'utilizzo di sistemi di archiviazione IT per la conservazione digitale

Di seguito i principi che dovrebbero essere rispettati nella progettazione o selezione di sistemi di archiviazione per la conservazione

\begin{tabular}{|c|c|}
\hline 1 & $\begin{array}{l}\text { Ridondanza e diversità } \\
\text { - Fare diverse copie indipendenti delle risorse digitali e archiviare in diversi luoghi } \\
\text { geografici. } \\
\text { - Usare una combinazione di sistemi di archiviazione online e di media offline. } \\
\text { - Usare tipi diversi di tecnologia di archiviazione per distribuire il rischio e } \\
\text { raggiungere un equilibrio per la sicurezza dei dati, un accesso facilitato e costi } \\
\text { gestibili. }\end{array}$ \\
\hline 2 & $\begin{array}{l}\text { Fixity, monitoraggio e riparazione } \\
\text { - Usare misure di fixity, come i checksum, per registrare e monitorare } \\
\text { regolarmente l'integrità di ogni copia della risorsa digitale. } \\
\text { - Se viene rilevato un danneggiamento o una perdita, allora usare una delle altre } \\
\text { copie per realizzare la sostituzione. } \\
\text { - Archiviare le informazioni di fixity insieme alle risorse digitali e separare anche i } \\
\text { database o i sistemi. }\end{array}$ \\
\hline 3 & $\begin{array}{l}\text { Osservazione della tecnologia e dei venditori, valutazione del rischio e migrazioni } \\
\text { proattive } \\
\text { - Rendersi conto che le tecnologie di archiviazione, prodotti e servizi hanno tutti } \\
\text { un ciclo di vita breve. } \\
\text { - Usare un sistema di monitoraggio della tecnologia per valutare il momento in cui } \\
\text { devono essere effettuati degli spostamenti. } \\
\text { - Fare attenzione alla fattibilità dell'archiviazione dei venditori o delle classi di } \\
\text { soluzioni di archiviazione. } \\
\text { - Essere proattivi nella migrazione di archiviazione prima che le risorse digitali } \\
\text { siano a rischio. }\end{array}$ \\
\hline 4 & $\begin{array}{l}\text { Consolidamento, semplicità, documentazione, provenienza e tracce di controllo } \\
\text { Minimizzare la proliferazione dei tipi di supporti obsoleti e sistemi di archiviazione usati } \\
\text { per la conservazione. } \\
\text { - Consolidare le risorse digitali sul minor numero d sistemi di archiviazione e } \\
\text { conservazione (soggetti ai requisiti di ridondanza di cui sopra). } \\
\text { - Documentare come sono state acquisite le risorse digitali e trasferite nei } \\
\text { sistemi di archiviazione e in che modo i sistemi di archiviazione e } \\
\text { conservazione sono impostati e gestiti. } \\
\text { - Usare ciò per fornire informazioni di audit sullautenticità dei dati. }\end{array}$ \\
\hline
\end{tabular}




\section{Affidabilità dell'archiviazione}

Quando si considerano delle soluzioni di archiviazione, che siano in loco o in cloud, si pone la questione di quanto siano affidabili e se qualcosa va male bisogna capire capirne le implicazioni in termini di perdita di dati. I soggetti produttori sosterranno generalmente le statistiche come l'affidabilità, durata, livelli di fallimento e di errore.

Per esempio, potrebbe trattarsi di un servizio cloud che viene progettato con una durata del 99.999\%, un Bit Error Rate (BER) di 1 su 10^16 quando si leggono i dati da un nastro di dati, o un Mean Time Between Failure (MTBF) di $1 \mathrm{M}$ di ore per l'unità rigida. Questi numeri vengono poi usati per calcolare altre misure di affidabilità, ad esempio potrebbe essere sostenuto il Mean Time To Data Loss (MTTDL) di 1,000 anni quando vengono usate le unità rigide in un array RAID6.

Questi numeri possono essere difficili da capire e devono essere interpretati con grande attenzione nel tentativo di calcolare 'quanto sicura' sarà una certa soluzione di archiviazione. Sono già stati fatti dei lavori importanti su come descrivere, calcolare e predire l'affidabilità dell'archiviazione, inclusi quelli che partono dal punto di vista della conservazione digitale. Si tratta di un argomento molto complicato e non verrà trattato in questo Manuale. Alcuni esempi di riferimenti per ulteriori suggerimenti sono Greenan et al (2010), Rosenthal (2010) and Elerath (2009). Da questo lavoro si possono trarre diverse considerazioni importanti:

- Le tecnologie di archiviazione IT sono generalmente affidabili per le funzioni che svolgono. Gli insuccessi rappresentano eventi piuttosto rari, tuttavia esistono e possono capitare. Si tende a pensare che solo perché a livello personale non si è mai andati incontro a un certo tipo di fallimento, le tecnologie di archiviazione siano in linea generale più affidabili di quanto esse siano in realtà, e questa è una condizione pericolosa. Per esempio, diversa gente sarà in possesso di dischi rigidi che hanno perfettamente funzionato per anni ed anni, ma la verità è che, in media, fino al 5-15\% i dischi rigidi si rompono nel giro di un anno (Backblaze, 2014), (Pinheiro et al, 2007).

- Dal momento che insuccessi ed errori rappresentano degli eventi rari, le statistiche di affidabilità da parte dei venditori sono generalmente basate su modelli e simulazioni e non su osservazioni a lungo termine di ciò che realmente si verifica nella pratica. Per esempio, se un produttore sostiene che il periodo di validità dei media è di 30 anni, non è dovuto al fatto che questi abbiano realmente testato i media su quel periodo di tempo. Allo stesso modo, se un venditore stima che MTTDL è di 1.000 anni, chiaramente non vuol dire che abbiano costruito un sistema e testato per tutta la durata di quell'intervallo di tempo. Per cui, le statistiche dovrebbero essere interpretate come strumenti che valutano al meglio da parte dei venditori in che modo un sistema dovrebbe comportarsi nella pratica - anche se poi potrebbe non verificarsi. Ad esempio, studi specifici hanno rilevato che la valutazione del produttore sull'affidabilità può risultare eccessivamente ottimista (Jiang et al, 2008).

- La probabilità che si verifichi una perdita di dati aumenta considerevolmente quando vengono tenute in conto le correlazioni. Le correlazioni riguardano quelle parti di un 
sistema, o diverse copie delle risorse digitali, che non possono essere considerate in modo indipendente. Se si riscontra un problema con una parte del sistema o copia delle risorse digitali, allora è probabile che si tratti di problemi relativi ad un'altra parte o alla copia. Gli esempi riguardano i difetti di fabbricazione che colpiscono tutti i dischi rigidi presenti nel server di archiviazione, errori di programmi software o firmware che danneggiano sistematicamente le risorse digitali, fallimenti da parte di un'organizzazione nel testare i suoi backup con regolarità o nell'isolare o dissociare i sistemi di archiviazione in modo che se una copia delle risorse digitali viene accidentalmente cancellata, le altre copie non vengano rimosse. I fattori di correlazione possono essere ben più significativi delle specifiche modalità di insuccesso trattate dalla statistica di affidabilità.

Questi risultati e queste osservazioni si trovano nelle seguenti raccomandazioni:

- Piano per gli insuccessi che si verificano nelle soluzioni di archiviazione IT indipendentemente da quanto ben progettate siano. Gli indici di fallimento nella pratica potrebbero essere più alti di quelli previsti dal produttore.

- La perdita dei dati può essere causata dall'insuccesso nel mettere in atto dei processi e delle procedure adeguate riguardo all'utilizzo dell'archiviazione IT, così come dalle stesse tecnologie di archiviazione. Un'adeguata valutazione del rischio rappresenta un modo per individuare e gestire questi problemi.

- La strategia migliore rimane quella di creare diverse copie indipendenti delle risorse digitali in luoghi differenti e archiviarli, ove possibile, utilizzando diverse tecnologie. Ciò dovrebbe comprendere un processo di verifica in modo attivo e regolare dell'integrità dei dati di tutte le copie in modo tale che si possano rilevare i problemi indipendentemente dal motivo e dal luogo in cui questi si riscontrano. In questo modo, i rischi vengono sia minimizzati che distribuiti, e l'affidamento non viene dato a nessuna tecnologia di archiviazione in particolare o servizio che sia completamente privo di errori.

\section{Strategie di archiviazione multi-copia}

Le tecnologie di archiviazione digitale presentano diversi rischi per la conservazione nel lungo periodo di oggetti digitali. Questi rischi possono essere ridotti grazie all'uso di una strategia di archiviazione digitale che coinvolge uno o più sistemi di archiviazione e almeno due copie dei dati.

Una strategia di archiviazione dovrebbe presentare queste caratteristiche:

(a) che esistano copie multiple indipendenti delle risorse digitali

(b) che queste copie siano geograficamente separate in luoghi diversi

(c) che le copie utilizzino diverse tecnologie di archiviazione

(d) che le copie utilizzino una combinazione di tecniche di archiviazione online e offline

(e) che l'archiviazione sia monitorata attivamente per far sì che qualsiasi problematica venga rilevata e risolta velocemente. 
Una strategia di archiviazione digitale può essere implementata a tappe, iniziando da un livello base di protezione e accesso del contenuto digitale e spostandosi poi verso un approccio più automatico e scalabile che garantisca un livello più alto di salvaguardia e sicurezza dei dati.

I rischi legati al contenuto digitale provengono da una serie di risorse e una strategia di archiviazione digitale fa da supporto al bilanciamento tra il costo dell'archiviazione digitale e la riduzione di quei rischi. Esempi di rischi da considerare comprendono l'incendio, l'inondazione, il fallimento nel promuovere o seguire dei processi o procedure idonei, attacchi malevoli, il degrado multimediale, e l'obsolescenza dei sistemi di archiviazione e di tecnologie. I rischi principali e gli strumenti per occuparsene e per mitigarli sono spesso oggetto della pianificazione della continuità aziendale in un'organizzazione (si veda Gestione del rischio e dei cambiamenti) Risulta importante comprendere che molti esempi relativi alla perdita del contenuto non sono necessariamente dovuti a difetti tecnici nella tecnologia di archiviazione (sebbene sia importante riconoscere che questi ultimi si verificano), ma possono essere provocati dall'errore umano, dalla mancanza di risorse o piani di migrazione di archiviazione, o da un fallimento relativo a un controllo regolare e a una correzione degli insuccessi che potrebbero avvenire.

In un mondo in cui l'uso di sistemi interconnessi e tecnologie di archiviazione digitale è sempre più in espansione, una copia offline delle risorse digitali assume un significato importante. Essa può fornire un 'tagliafuoco' rispetto a problematiche legate a sistemi online che possono propagarsi automaticamente tra le diverse posizioni, ad es., cancellazione di un file in una posizione che elimina automaticamente una copia speculare in un altro punto.

Creare più di una copia delle risorse digitali risulta fondamentale per raggiungere un livello base di protezione dei dati. Usare diverse tipologie di archiviazione per ogni copia aiuta a distribuire il rischio e garantire che una problematica riferita a una tecnologia non ne colpisca altre. II modo in cui ogni copia viene archiviata può essere adattato per raggiungere un livello complessivo accettabile di costo, rischio e complessità. Ad esempio, si può gestire una copia usando un server online di archiviazione per un accesso rapido e una copia potrebbe essere messa su nastro di dati in deep archive ${ }^{24}$ a prezzo contenuto e con un livello di sicurezza relativamente alto.

II Manuale segue i livelli di conservazione della National Digital Stewardship Alliance (NDSA) (NDSA, 2013), di seguito riportati, che raccomandano quattro livelli con cui la conservazione digitale può essere supportata attraverso ridondanza geografica e di archiviazione. Un'ulteriore raccomandazione è quella di utilizzare una combinazione di copie online e offline per arrivare ad avere una buona combinazione di accesso ai dati e sicurezza dei dati:

\footnotetext{
${ }^{24}$ Un deep archive è un punto di archiviazione di dati a cui è probabile non si possa accedere ancora, ma che deve essere mantenuto nel caso di verifica di conformità o per altre motivazioni aziendali.
} 


\begin{tabular}{|c|c|c|}
\hline Livello & Approccio & Rischi riscontrati e benefici ottenuti \\
\hline 1 & $\begin{array}{l}\text { Due copie complete delle risorse } \\
\text { digitali che non sono collocate. Una } \\
\text { copia dovrebbe essere offline. } \\
\text { Per quel che riguarda le risorse } \\
\text { digitali su media eterogenei (dischi } \\
\text { ottici, dischi rigidi, ecc.) bisogna } \\
\text { togliere il contenuto dal supporto e } \\
\text { inserirlo nel proprio sistema di } \\
\text { archiviazione. }\end{array}$ & $\begin{array}{l}\text { - Capacità di base di recupero in } \\
\text { seguito a diverse problematiche } \\
\text { che includono il fallimento del } \\
\text { sistema di archiviazione. Perdita o } \\
\text { danni a una copia che possono } \\
\text { essere recuperati utilizzandone } \\
\text { un'altra. } \\
\text { Risorse digitali più facili da gestire } \\
\text { quando si trovano in un sistema } \\
\text { singolo di archiviazione. }\end{array}$ \\
\hline 2 & $\begin{array}{l}\text { - Almeno tre copie complete } \\
\text { - } \text { Almeno una copia in una posizione } \\
\text { geografica diversa } \\
\text { Documentare i(I) propri(o) sistema/i } \\
\text { di archiviazione e supporti di } \\
\text { archiviazione, e ciò di cui si ha } \\
\text { bisogno per utilizzarli. }\end{array}$ & $\begin{array}{l}\text { - Come sopra e in più una protezione } \\
\text { aggiuntiva contro disastri naturali e } \\
\text { altri eventi di forza maggiore. } \\
\text { Buon livello di accesso e sicurezza } \\
\text { delle risorse digitali. } \\
\text { - II personale dispone di politiche e } \\
\text { procedure chiare da seguire che } \\
\text { risultano così più efficaci, i costi } \\
\text { sono ridotti e i cambiamenti nel } \\
\text { personale possono essere gestiti. }\end{array}$ \\
\hline 3 & $\begin{array}{l}\text { - Almeno una copia in una posizione } \\
\text { geografica che presenta una forma } \\
\text { diversa di minaccia naturale. } \\
\text { Monitoraggio dell'obsolescenza e } \\
\text { processo di migrazione per il/i } \\
\text { proprio/i sistema/i di archiviazione e } \\
\text { media. }\end{array}$ & $\begin{array}{l}\text { - Come sopra e in pù una maggiore } \\
\text { protezione per i rischi a lungo } \\
\text { termine associati all'obsolescenza } \\
\text { tecnica. } \\
\text { - L'accesso continuo al contenuto è } \\
\text { possibile anche durante gli } \\
\text { spostamenti o i disastri. }\end{array}$ \\
\hline 4 & $\begin{array}{l}\text { - Almeno una copia in una posizione } \\
\text { geografica presenta una forma } \\
\text { diversa di minaccia naturale. } \\
\text { Disporre di un piano completo che } \\
\text { manterrà i file e i metadati su media } \\
\text { o sistemi attualmente accessibili. }\end{array}$ & $\begin{array}{l}\text { - Come sopra e in più una serie } \\
\text { completa di rischi, inclusi la perdita } \\
\text { accidentale e gli attacchi malevoli, il } \\
\text { blocco da fornitore, e l'instabilità di } \\
\text { bilancio. } \\
\text { - Il contenuto è altamente disponibile, } \\
\text { i costi prevedibili e gestibili, si ha la } \\
\text { capacità di ottenere una } \\
\text { certificazione affidabile di } \\
\text { repository. }\end{array}$ \\
\hline
\end{tabular}




\section{Gestione dell'obsolescenza dei sistemi di storage e rischi}

L'impiego di tecnologie e soluzioni di archiviazione deve essere accuratamente pianificato e gestito per rappresentare un approccio efficace di supporto alla conservazione digitale. Se fatto in modo adeguato, il risultato può raggiungere dei livelli davvero alti per la sicurezza dei dati, per l'accesso veloce al contenuto quando richiesto, e i costi sia bassi che prevedibili.

È possibile che le tecnologie di archiviazione non abbiano successo o possano danneggiare i dati, e che il ciclo di vita dei media e dei sistemi sia generalmente breve, ad esempio 3-5 anni, il che significa che le soluzioni sono diventate obsolete velocemente e che se ne richiede una migrazione per evitare che le risorse digitali possano essere a rischio.

Migrare in questo contesto significa spostare i dati da un vecchio sistema di archiviazione ad uno nuovo. Le risorse digitali stesse non cambiano, quello che cambia è la soluzione di archiviazione. Una struttura IT o un fornitore di servizi di archiviazione quando ragionerà sulla migrazione lo farà a livello di archiviazione. Ciò è in contrasto con la migrazione del formato dei file in cui è il formato dei file a subire un cambiamento mentre non viene modificato il modo in cui i file vengono archiviati.

\section{Risorse}

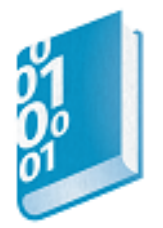

\section{NDSA Levels of Preservation}

http://www.digitalpreservation.gov/ndsa/activities/levels.html (2013)

https://ndsa.org//publications/levels-of-digital-preservation/ (Version 2.0, 2018)

La National Digital Stewardship Alliance (NDSA) "Levels of Digital Preservation" costituisce un insieme di raccomandazioni multilivello su come le organizzazioni dovrebbero iniziare a costruire o migliorare le loro attività di conservazione digitale. Mira a rappresentare un set di linee guida di uso relativamente facile non solo per chi inizia a considerare l'ipotesi di conservare i propri contenuti digitali, ma anche per gli enti che pianificano i prossimi passaggi in prospettiva di un miglioramento dei loro sistemi di conservazione digitale attuali e dei flussi di lavoro. Non è soltanto destinato alla valutazione della solidità dei programmi di conservazione digitale nella loro interezza, ma riguarda anche aspetti relativi alle politiche, al personale e al supporto organizzativo.

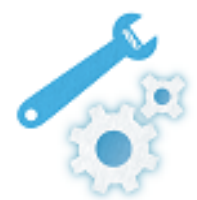

Di seguito alcuni dei più noti sistemi di archiviazione e conservazione digitale e di fornitori di sistemi/servizi di archiviazione. Vi sono diversi fornitori di prodotti di archiviazione IT, così come fornitori professionisti di servizi relativi alla conservazione digitale che possono garantire un 
sistema di archiviazione in loco o in ambiente cloud (si veda Servizi Cloud). Generalmente, questi professionisti potrebbero supportare altre funzioni di conservazione oltre all'archiviazione.

\section{Arkivum}

http://arkivum.com

Digital Preservation Network

http://www.dpn.org

DSpace

http://www.dspace.org

ePrints

http://www.eprints.org

Fedora

http://fedorarepository.org

iRods

http://irods.org

\section{LOCKSS}

http://www.lockss.org

OCLC Digital Archive CONTENTdm

http://www.oclc.org/digital-archive.en.html

\section{Portico}

http://www.portico.org/digital-preservation/

\section{Preservica}

http://preservica.com

\section{Rosetta}

https://www.exlibrisgroup.com/products/rosetta-digital-asset-management-and-preservation/

\section{Community Owned digital Preservation Tools Registry COPTR}

http://coptr.digipres.org/Main Page

Sebbene sia principalmente incentrato sugli strumenti, il registry COPTR riguarda anche una serie di sistemi e servizi di archiviazione. Rappresenta un sistema di individuazione e valutazione che supporta i professionisti del settore a trovare uno strumento di cui hanno bisogno per la conservazione dei loro dati digitali. COPTR rileva i dettagli basici e pratici di un 
tool, le funzioni del tool, e la modalità per trovare più informazioni (URL rilevanti) e riferimenti per le esperienze degli utenti con il tool.

WWW

DSHR's Blog

http://blog.dshr.org

David Rosenthal è un informatico ed è il direttore scientifico del progetto LOCKSS. II suo blog tratta di frequente temi legati allo sviluppo dell'archiviazione informatica e i trend e le implicazioni della conservazione digitale.

Casi di studio

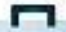

D)

The National Archives case study: Bodleian Library, University of Oxford

http://www.nationalarchives.gov.uk/documents/archives/case-study-oxford.pdf

Questo caso di studio riguarda la Biblioteca Bodleian e l'Università di Oxford e la disposizione di un'infrastruttura locale di 'cloud privato' per le loro collezioni digitali, compresi i libri elettronici, immagini e multimedia, dati della ricerca e cataloghi. Descrive il contesto organizzativo, la natura dei suoi requisiti di conservazione digitale e gli approcci, i suoi servizi di archiviazione, le infrastrutture tecniche, il caso aziendale e i finanziamenti. Si conclude con lezioni chiave apprese e progetti futuri. Gennaio 2015 (4 pagine).

The National Archives case study: Parliamentary Archives

http://www.nationalarchives.gov.uk/documents/archives/case-study-parliament.pdf

Questo caso di studio riguarda gli Archivi Parlamentari. È un esempio di un archivio che utilizza un insieme ibrido di soluzioni di archiviazione (in parte cloud pubbliche e in parte installate localmente) per la conservazione digitale dal momento che l'archivio dispone di un sistema di conservazione installato localmente (Preservica Enterprise Edition) che è integrato con l'archiviazione cloud e locale e sta archiviando localmente, non in cloud, risorse riservate. Gennaio 2015 (4 pagine).

The National Archives case study: Tate Gallery

http://www.nationalarchives.gov.uk/documents/archives/case-study-tate-gallery.pdf

Questo caso di studio descrive l'esperienza riferita allo sviluppo di un archivio digitale condiviso per le quattro postazioni della Tate alimentate da un sistema di archiviazione commerciale di Arkivum. Descrive il contesto organizzativo, la natura dei suoi requisiti di conservazione digitale e gli approcci, e la logica di selezione in loco di Arkivum, "Arkivum/OnSite", rispetto a qualsiasi altro prodotto basato sul cloud. Si conclude con lezioni chiave apprese e progetti futuri. Gennaio 2015 (4 pagine). 


\section{Riferimenti}

Backblaze, 2014. Hard Drive Reliability Update - Sep 2014. Backblaze. [blog] Disponibile al seguente link: https://www.backblaze.com/blog/hard-drive-reliability-update-september-2014/ Elerath, J., 2009. Hard-Disk Drives: The Good, the Bad, and the Ugly. Communications of the ACM. 52 (6), 38-45. Disponibile al seguente link: doi:10.1145/1516046.1516059. http://cacm.acm.org/magazines/2009/6/28493-hard-disk-drives-the-good-the-bad-and-the$\underline{\text { ugly/fulltext }}$

Greenan, K.M., Plank, J.S. \& Wylie, J.J., 2010.Mean time to meaningless: MTTDL, Markov models, and storage system reliability. Proceedings of the 2nd USENIX conference on Hot topics in storage and file systems. Disponibile al seguente link: https://www.usenix.org/legacy/event/hotstorage10/tech/full papers/Greenan.pdf

Jiang, W. et al., 2008. Are Disks the Dominant Contributor for Storage Failures? A Comprehensive Study of Storage Subsystem Failure Characteristics. Proceedings of the 6th USENIX Conference on File and Storage Technologies (FAST '08). Disponibile al seguente link: http://www.usenix.org/events/fast08/tech/jiang.html

NDSA, 2013. The NDSA Levels of Digital Preservation: An Explanation and Uses, version 1 2013. National Digital Stewardship Alliance. Disponibile al seguente link: http://www.digitalpreservation.gov/ndsa/working groups/documents/NDSA Levels Archiving 2013.pdf

Pinheiro, P., Weber, W-D. \& Barroso, L.A., 2007. Failure Trends in a Large Disk Drive Population. Proceedings of the 5th USENIX Conference on File and Storage Technologies (FAST' 07). Disponibile al link: http://static.googleusercontent.com/media/research.google.com/en//archive/disk failures.pdf

Rosenthal, D.S.H., 2010. Bit Preservation: A Solved Problem? The International Journal of Digital Curation. 5 (1) Stanford University Libraries, CA. Disponibile al seguente link: http://www.ijdc.net/index.php/ijdc/article/view/151/224 


\section{Supporti obsoleti}

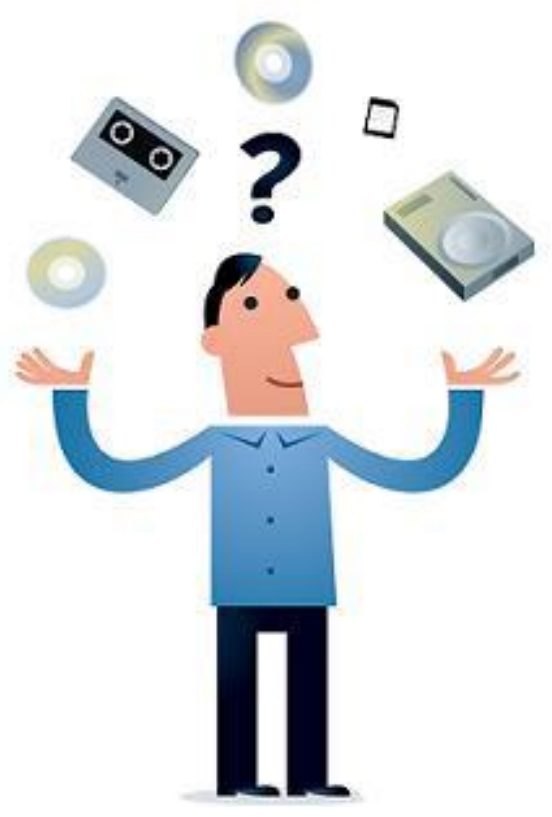

Illustrazione di Jørgen Stamp digitalbevaring.dk CC BY 2.5 Denmark

\section{Introduzione}

Molte organizzazioni si troveranno con grandi quantità di dati archiviati su supporti obsoleti, come i media magnetici ed ottici, e i dati continueranno ad essere ricevuti su vecchi supporti. In definitiva, la migliore strategia a lungo termine per la conservazione dei dati sarà la loro migrazione verso archivi basati sui file e successivamente verso una gestione attiva (si veda la sezione Archiviazione). Allo stesso tempo, i media originali saranno ancora utilizzati per diverso tempo, perciò si dovranno comprendere i loro processi di conservazione e requisiti di archiviazione. Per quelle organizzazioni che dispongono di grandi collezioni di supporti obsoleti, capire i rischi che ogni tipologia di media corre aiuterà anche a dare priorità alle collezioni per la migrazione e l'applicazione di strumenti e metodi di informatica forense (si veda la sezione Informatica forense).

Per la conservazione di media magnetici e ottici si devono considerare due aspetti - gli stessi media e i sistemi hardware e software necessari per l'interpretazione. In alcuni casi, il secondo aspetto rappresenterà quello più difficile. Considerato che la popolarità di un formato di media tende a decadere, la realizzazione hardware si interrompe e diventa più difficile da ottenere $\mathrm{e}$ mantenere.

\section{Conservare i supporti obsoleti}

Nella maggior parte dei casi il modo più semplice per ridurre le possibilità di rischi relativi ai supporti di archiviazione è quello di trasferire tutto il contenuto in un sistema di archiviazione organizzato. Ciò significa che il contenuto può essere gestito senza riferirsi al mezzo di archiviazione originario, e potrebbe essere appropriato per la maggior parte dei contenuti digitali 
per cui è necessaria la conservazione. Tuttavia, ci possono essere situazioni in cui è necessario mantenere in qualche modo i supporti originali. In alcuni casi, il mezzo di archiviazione potrebbe semplicemente essere mantenuto come un artefatto, senza aspettarsi un accesso a lungo termine, ad es., dove esso fa parte di una collezione ibrida o ha qualche tipo di valore per associazione (ad es., parte delle collezioni di un autore importante). Tuttavia, laddove è richiesto un accesso costante al contenuto, bisogna riflettere attentamente su come quest'ultimo possa essere accessibile in futuro.

Una cosa che abbiamo imparato dall'esperienza è che i tipi di supporto di archiviazione digitale cambiano di frequente nel tempo. Per esempio, la versione precedente di questo Manuale conteneva una panoramica dei supporti di archiviazione e forniva stime sulla durata di determinati tipi di supporti di archiviazione che erano in voga nella metà degli anni '90 (un manuale di conservazione digitale scritto decenni fa avrebbe dovuto includere presumibilmente delle valutazioni di schede perforate e nastri cartacei). Alla luce delle tendenze attuali nella tecnologia di archiviazione, probabilmente è meglio fornire ad oggi una struttura che possa supportare la valutazione attuale dei supporti di archiviazione, che ora potrebbero includere chiavette USB o dischi rigidi esterni. Una struttura del genere è stata offerta dal National Archives (Brown, 2008) che usa un approccio basato su scheda di punteggio, misurando determinati supporti di archiviazione in riferimento a sei criteri:

- longevità (ad es., durata operativa provata)

- capacità

- fattibilità (ad es., in termini di mantenimento dell'integrità probatoria)

- obsolescenza

- costi

- $\quad$ suscettibilità (ad es., ai danni fisici e alle diverse condizioni ambientali)

Nella pratica, comunque, questi tipi di valutazioni non arrivano che a questo punto. Ci sono sempre più elementi probatori che indicano il ruolo importante rivestito anche dalla variazione nella qualità di realizzazione nella longevità dei media (Harvey, 2011). Questa è la ragione per cui alla fine la conservazione digitale dipende dal trasferimento del contenuto dai media verso un ambiente organizzato di archiviazione.

\section{Risorse

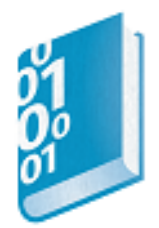

Selecting storage media for long-term preservation, TNA Digital Preservation Guidance Note 2: August 2008

https://www.nationalarchives.gov.uk/documents/selecting-storage-media.pdf

Questo documento fa parte di un insieme di note orientative realizzate dal National Archives e presenta delle raccomandazioni generali sugli aspetti relativi alla conservazione e alla gestione dei documenti elettronici. Si rivolge a chiunque sia coinvolto nella creazione di contenuti 
elettronici. Fornisce informazioni ai creatori e gestori dei documenti elettronici sulla selezione dei supporti di archiviazione fisici nell'ambito della conservazione a lungo termine. Si tenga presente che la guida è valida a partire da agosto 2008. (7 pagine).

Care, Handling and Storage of Removable media, TNA Digital Preservation Guidance Note 3: August 2008

http://www.nationalarchives.gov.uk/documents/information-management/removable-media-

care.pdf

Questo documento fa parte di un insieme di note orientative realizzate dal National Archives e presenta delle raccomandazioni generali sugli aspetti relativi alla conservazione e alla gestione dei documenti elettronici. Riguarda la cura, la gestione e l'archiviazione di supporti di archiviazione rimovibili. Si tenga presente che la guida è valida a partire da agosto 2008. (10 pagine).

You've Got to Walk Before You Can Run: First Steps for Managing Born-Digital Content Received on Physical Media

http://www.oclc.org/content/dam/research/publications/library/2012/2012-06.pdf

Una guida passo dopo passo su come recuperare le risorse native digitali dai vari supporti fisici. Il focus è sull'identificazione e stabilizzazione delle collezioni in modo che ci si possa trovare nella posizione di intraprendere altri passaggi allorquando le risorse, le competenze e il tempo lo consentiranno. II documento sul progetto POWRR Resources for Technical Steps integra ulteriori risorse per alcuni passaggi. (7 pagine).

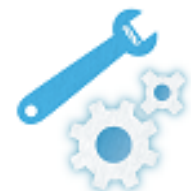

Kryoflux: Commercial tools for reading floppy disks

http://www.kryoflux.com/

KryoFlux è un dispositivo USB specificamente progettato per acquisire una lettura di basso livello attendibile idonea per la conservazione del software. È un hardware ufficiale sviluppato dalla Software Preservation Society.

WWW

Digital Preservation Management: Chamber of Horrors

http://dpworkshop.org/dpm-eng/oldmedia/disks.html

Alcuni esempi di supporti obsoleti e a rischio.

\section{Lost Formats}

http://www.experimentaljetset.nl/archive/lostformats

Pagina web della Lost Formats Preservation Society con una rassegna dei profili che permettono di avere una rapida identificazione, una breve cronologia di riferimento e caratteristiche come dimensioni e capacità di archiviazione. Tutti i profili, anziché aumentare di 
proporzioni, si presentano simili nelle dimensioni. Gli ultimi principali aggiornamenti sembrano datati 2008, ma il contenuto è sempre valido per tutti i formati, ad eccezione dei più recenti.

\section{Museum of Obsolete Media \\ http://www.obsoletemedia.org/category/format/}

Un'ottima risorsa che riguarda un grande insieme di supporti di archiviazione obsoleti audio, video, dati e film. La navigazione può essere effettuata tramite delle categorie o sulla Galleria e Timeline. Risulta utile se si sa già ciò che si sta cercando, molto proviene dalle voci di Wikipedia.

\section{Casi di studio}

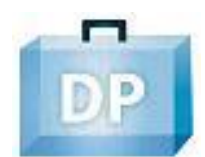

\section{A Fistful of Floppies: Digital Preservation in Action}

https://ischool.uw.edu/sites/default/files/capstone/posters/JStanley Capstone Landscape.pdf II sistema bibliotecario dell'Università di Washington dispone attualmente di un numero limitato di tesi elettroniche integrando le risorse dalla fine degli anni '80 al 2011 sui floppy disk e CDrom. Queste risorse raggiungeranno presto, o lo hanno già fatto, il limite della loro durata prevista. Questo progetto del 2015 descrive le possibilità della conservazione digitale per questa collezione di risorse utilizzando l'informatica forense come modello. (1 pagina).

Enford, D., et al 2008, Media Matters: developing processes for preserving digital objects on physical carriers at the National Library of Australia, Papers from 74th IFLA General Conference and Council

http://archive.ifla.org/IV/ifla74/papers/084-Webb-en.pdf

La National Library australiana dispone di una piccola ma importante collezione di risorse digitali su supporti fisici, comprese sia le risorse pubblicate che i manoscritti non pubblicati in forma digitale. II Digital Preservation Workflow Project ha lo scopo di realizzare un processo scalabile semi-automatico per il trasferimento dei dati da supporti fisici a sistemi di archiviazione digitali di massa per la conservazione, favorendo la mitigazione dei rischi associati ai supporti fisici. (17 pagine).

\section{Digital Preservation Planning Case Study}

http://www.dpconline.org/component/docman/doc download/863-2013-may-getting-started-

\section{london-planning-case-study-ed-fay}

Una presentazione su come iniziare una pianificazione sulla conservazione digitale, tra cui rientrano le attività di individuazione degli obiettivi, la valutazione del rischio e l'assegnazione di priorità alle proprie collezioni (inclusi gli esempi di supporti obsoleti), e i ruoli e le responsabilità del personale. 2013 (20 pagine). 


\section{Riferimenti}

Brown, A., 2008. Selecting storage media for long-term preservation. TNA Digital Preservation Guidance Note 2: August 2008. Disponibile al seguente link: https://www.nationalarchives.gov.uk/documents/selecting-storage-media.pdf

Harvey, R., 2011. Preserving Digital Materials 2nd edition. De Gruyter Saur. 


\section{Piano di conservazione}

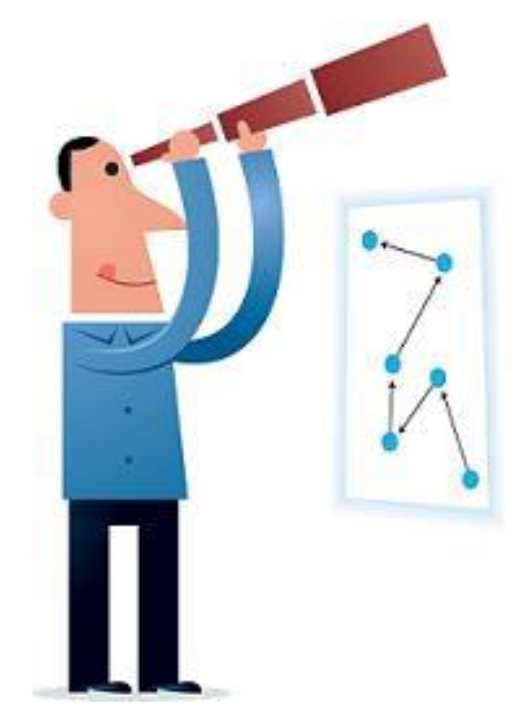

Illustrazione di Jørgen Stamp digitalbevaring.dk CC BY 2.5 Denmark

Cos'è il piano di conservazione?

Il piano di conservazione all'interno di un repository digitale serve per il monitoraggio dei cambiamenti che potrebbero incidere sulla sostenibilità delle risorse digitali presenti o sul loro accesso. Questo processo dovrebbe essere proattivo sia allo stato attuale che in prospettiva, in termini di acquisizioni e tendenze. I cambiamenti potrebbero verificarsi all'interno del repository, all'interno dell'organizzazione in cui si trova il repository o all'esterno del repository e delle organizzazioni stesse. Questi ultimi possono essere monitorati nelle seguenti aree:

- Monitoraggio tecnologico

- costruzione dei pacchetti

- archiviazione

o formati

○ strumenti

○ ambiente

- meccanismi di accesso

- Comunità designate

- necessità e aspettative degli utenti

- necessità e aspettative dei produttori

- strumenti emergenti per l'accesso ai sistemi

- riscontro formale da utenti e produttori

Il concetto di piano di conservazione viene definito nel modello funzionale dello standard $\underline{\mathrm{OAIS}}$ (CCSDS, 2012). Questa sezione si concentra soprattutto sui componenti di Monitoraggio inclusi 
nella definizione OAIS. Le funzioni OAIS 'Monitor Technology' e 'Monitor Designated Community' offrono informazioni sulle attività del piano di conservazione che segnalano al repository i cambiamenti nell'ambiente esterno e i rischi che potrebbero avere un effetto sulla sua capacità di conservare e mantenere l'accesso alle informazioni sulle quali ha il controllo, come le innovazioni nelle tecnologie di archiviazione e accesso, o cambiamenti nel campo di applicazione o aspettative della Comunità designata (si veda Lavoie, 2015,13). II piano di conservazione presenta poi delle raccomandazioni per l'aggiornamento delle politiche e procedure del repository al fine di incorporare questi cambiamenti. La funzione del piano di conservazione rappresenta una strategia di protezione per l'OAIS dall'ambiente in continua evoluzione delle tecnologie e degli utenti. Rileva i cambiamenti e i rischi che hanno un effetto sulla capacità del repository di rispettare le sue responsabilità, progetta delle strategie per occuparsene, e supporta l'implementazione di queste strategie nel sistema d'archivio.

\section{Qual è lo scopo del piano di conservazione?}

\section{Identificazione dei fattori per avviare la conservazione delle risorse digitali}

Una volta identificati i cambiamenti, si può implementare un processo di valutazione del rischio per analizzare e individuare il cambiamento che costituisce un pericolo significativo per le risorse digitali nel repository. I rischi possono poi essere affrontati e auspicabilmente mitigati seguendo un'attività relativa al piano di conservazione per decidere l'azione adeguata di conservazione. In questo caso il processo di monitoraggio e osservazione tecnologica va ad individuare i punti soglia per ulteriori analisi, per il piano di conservazione, e, laddove sia importante, per le azioni per conservare le risorse digitali.

\section{Costruzione di una base di conoscenza per comunicare le attività di conservazione} Il processo di monitoraggio di fattori interni ed esterni come parte dell'attività del piano di conservazione può fornire informazioni sulla base di conoscenza di un'organizzazione, e, così facendo, migliorare la sua capacità di portare avanti efficacemente le attività di conservazione digitale. Per esempio, la 'base di conoscenza' di un'organizzazione potrebbe essere migliorata con l'apporto di informazioni sulle prestazioni di un nuovo software o sull'obsolescenza e indisponibilità di uno strumento esistente. In qualche caso questo processo può essere eseguito al meglio in modo individuale o all'interno di un'organizzazione, ma in alternativa potrebbe essere più utile se eseguito in modo collaborativo. L'ampiezza dell'ambito della conoscenza prevista per la conservazione digitale fa optare naturalmente per l'approccio collaborativo, per mezzo del quale alcune organizzazioni sono in grado di specializzarsi in aree specifiche e far sì che le conoscenze siano aperte e condivise.

\section{Implementazione del servizio di pianificazione della conservazione}

II livello al quale risulterà necessario il monitoraggio della tecnologia varierà a seconda del grado di uniformità o controllo in base al quale i formati e i media possono essere implementati dall'istituzione. Le istituzioni con un controllo più basso sui media e sui formati ricevuti e con un elevato livello di diversità nelle loro collezioni riterranno essenziale questa funzionalità. Per la maggior parte degli enti, la strategia IS dovrebbe mirare a sviluppare degli standard aziendali in 
modo che chiunque possa utilizzare lo stesso software e le stesse versioni ed essere rinviato verso le nuove versioni nel momento in cui i prodotti vengono aggiornati.

L'insuccesso dell'implementazione di un monitoraggio tecnologico efficace e di una strategia IS che la integri potrebbe comportare una perdita di accesso alle collezioni digitali e costi più alti. Ad esempio, può capitare che si possa ristabilire l'accesso attraverso l'informatica forense (si veda Informatica forense), ma potrebbe essere costoso rispetto alle strategie di prelazione.

Un'indagine retrospettiva delle collezioni digitali (si veda Guida introduttiva) e una valutazione del rischio e un piano d'azione (si veda Gestione del rischio e del cambiamento) potrebbero essere dei primi passaggi necessari per diversi enti, preliminari all'implementazione del monitoraggio tecnologico.

Una corretta conservazione dei metadati in un catalogo digitale che individua il mezzo di archiviazione, l'hardware necessario, il sistema operativo e il software, consentirà una strategia di monitoraggio tecnologico (si veda Metadati e documentazione).

Anche i sistemi di conservazione integrati, i singoli strumenti e i registry possono supportare questa funzione (si veda Soluzioni tecniche e strumenti).

\section{Risorse}

Alcune delle principali attività di monitoraggio della conservazione sono generali, e, pertanto, già adatte ad una collaborazione, mentre altre sono specificamente circoscritte, e, perciò, non facilmente condivisibili.

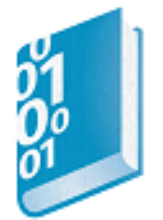

\section{DPC Technology Watch Report Series}

http://www.dpconline.org/advice/technology-watch-reports

Questi report presentano un'introduzione avanzata su aspetti specifici per chi ha il compito di definire e implementare servizi per la conservazione a lungo termine e per l'accesso. I report sono aggiornati e ne vengono aggiunti periodicamente di nuovi.

\section{Scout - a preservation watch system, OPF blog post 16th Dec 2013 \\ http://openpreservation.org/blog/2013/12/16/scout-preservation-watch-system/}

II progetto SCAPE ha ideato un dimostratore, chiamato SCOUT, per un servizio di monitoraggio automatico della conservazione. SCOUT è stato descritto dai suoi sviluppatori come uno strumento che fornisce "...una base di conoscenza ontologica per centralizzare tutte le informazioni necessarie per rilevare i rischi legati alla conservazione e le opportunità. Usa plugin per permettere un'integrazione facilitata di nuove fonti di informazioni, come ad esempio i registry dei formati dei file, strumenti per la caratterizzazione, migrazione e garanzia della qualità, politiche e conoscenza, e altro". 
Assessing file format risks: searching for Bigfoot? OPF Blog post 29th Oct 2014 http://openpreservation.org/blog/2013/09/30/assessing-file-format-risks-searching-bigfoot/

Questo post dettagliato su blog desta preoccupazione sulle sfide relative al monitoraggio automatico della conservazione.

Barbara Sierman, Paul Wheatley 2010 Evaluation of Preservation Planning within OAIS, based on the Planets Functional Model Planets Deliverable no. PP7-D6.1

http://www.planets-project.eu/docs/reports/Planets PP7-D6 EvaluationOfPPWithinOAIS.pdf

II progetto Planets ha analizzato vari aspetti dei concetti definiti all'interno della funzione OAIS 'Piano di Conservazione', ed eseguito una valutazione di OAIS basandosi su queste esperienze pratiche. 2010 (34 pagine).

\section{Community Owned digital Preservation Tools Registry COPTR}

http://coptr.digipres.org/Main Page

COPTR descrive gli strumenti utili per la conservazione digitale a lungo termine e si può considerare principalmente un mezzo di identificazione e valutazione per aiutare i professionisti del settore a trovare gli strumenti di cui hanno bisogno per la conservazione dei dati digitali. COPTR ha lo scopo di collezionare in un unico luogo la conoscenza della comunità dell'ambito della conservazione digitale sugli strumenti di conservazione. Inizialmente comprendeva registry di tipi di dati delle organizzazioni partner di COPTR, inclusi quelli gestiti dal Digital Curation Centre, Digital Curation Exchange, National Digital Stewardship Alliance, Open Preservation Foundation, e Preserving digital Objects With Restricted Resources project (POWRR). COPTR rileva i dettagli di base e pratici di uno strumento, identifica la sua funzionalità e la modalità con cui trovare più informazioni (URL rilevanti) e riferimenti per le esperienze degli utenti con il tool. L'ambito si riferisce all'accezione generale del termine 'conservazione digitale'. In altre parole, se si ritiene che uno strumento sia utile per l'esecuzione di funzionalità di conservazione digitale, come quelle descritte nel modello OAIS o nel modello del ciclo di vita DCC, allora esso rientra nell'ambito di questo registry.

Casi di studio

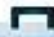

D) D

OCLC Research Report - Preservation Health Check: Monitoring Threats to Digital Repository Content

http://www.oclc.org/research/themes/research-collections/phc.html

L'attività dell'OCLC Research Preservation Health Check è stata avviata dalla Open Planets Foundation. II Pilot ha usato un campione di metadati di conservazione forniti dalla Bibliothèque Nationale de France. II report presenta i risultati preliminari della Fase 1 del Pilot e sottolinea la possibilità di utilizzare i metadati di conservazione PREMIS come principio probatorio per supportare un'attività di valutazione del rischio basata sul modello Simple Property-Oriented Threat (SPOT). 


\section{Digital Preservation Planning Case Study}

http://www.dpconline.org/component/docman/doc download/863-2013-may-getting-started-

london-planning-case-study-ed-fay

Una presentazione su come avviare un piano di conservazione digitale, comprende le attività di individuazione degli obiettivi, la valutazione del rischio e l'assegnazione di priorità alle proprie collezioni (inclusi gli esempi di supporti obsoleti), e i ruoli e le responsabilità del personale. 2013 (20 pagine).

\section{Riferimenti}

Consultative Committee for Space Data Systems, 2012. Reference model for an open archival information system (OAIS): Recommended practice (CCSDS 650.0-M-2: Magenta Book), CCSDS, Washington, DC. Disponibile al seguente link: https://public.ccsds.org/pubs/650x0m2.pdf

(Note this is a free to download version of ISO 14721:2012, Space Data and Information Transfer Systems - Open Archival Information System (OAIS) - Reference Model, 2nd edn). Lavoie, B., 2014. The Open Archival Information System (OAIS) Reference Model: Introductory Guide (2nd Edition) DPC Technology Watch Report 14-02 October 2014. Disponibile al seguente link: http://dx.doi.org/10.7207/TWR14-02 


\section{Azione di Conservazione}

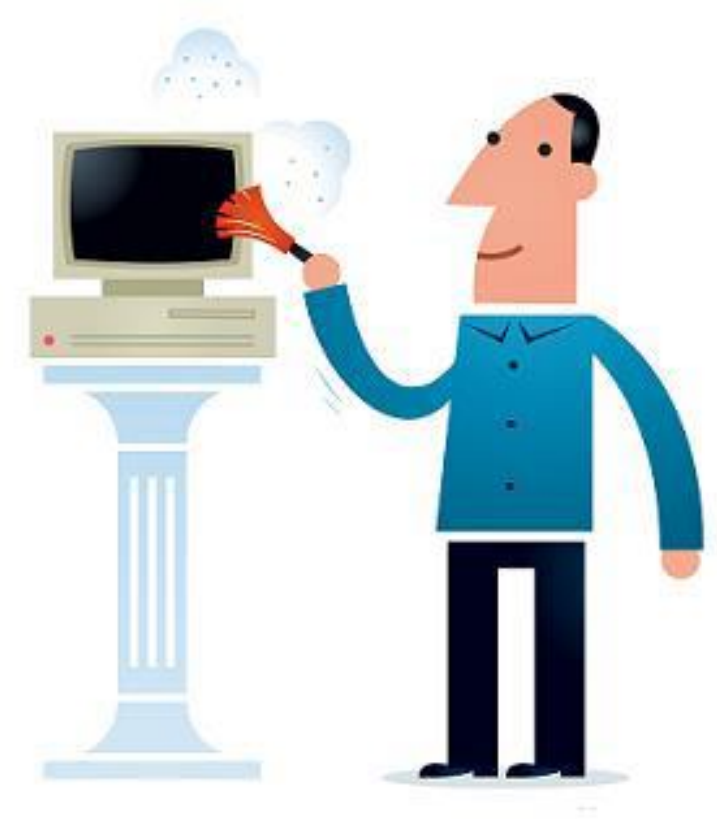

Illustrazione di Jørgen Stamp digitalbevaring.dk CC BY 2.5 Denmark

\section{Introduzione}

Fino ad adesso abbiamo imparato che la conservazione digitale consiste in una serie di sfide provenienti dall'ambito organizzativo, amministrativo, culturale e tecnico e da quello relativo alle risorse.

Questa sezione del Manuale si concentrerà, nello specifico, sulle azioni che possono essere intraprese per aiutare a mitigare le problematiche tecniche di conservazione di risorse digitali nel tempo.

\section{Obsolescenza tecnologica dei formati}

L'obsolescenza tecnologica ha da tanto tempo rappresentato un problema significativo per la conservazione digitale. Tuttavia, studi recenti hanno indicato che l'obsolescenza dei formati non è sempre così diffusa come si teme (Rosenthal 2015a, Jackson 2012), è una questione da tenere in conto e da contrastare qualora le risorse digitali debbano sopravvivere per generazioni di cambiamenti tecnologici, ma di sicuro non esiste solo questo problema. Tuttora esistono molti formati dei file consolidati, sono supportati e utilizzabili. È molto probabile che, anziché obsoleta, la maggior parte dei formati file con cui ci si potrebbe interfacciare sarà generalmente comprensibile e ben supportata.

Una definizione semplice di obsolescenza è il processo per cui si diventa datati o non più utilizzati. Quando si parla di obsolescenza tecnologica ci si riferisce a 'questo software Wordperfect 3.1 è obsoleto' o 'questo Micro computer BBC è obsoleto'. È difficile stabilire il 
momento esatto in cui si verifica l'obsolescenza, in particolare per le risorse che sono divenute obsolete solo di recente. Per esempio, soltanto perché l'applicazione originale (ad es., MS Word) non supporta più un certo formato non significa che nessun altro software che è capace di leggere il formato non sia disponibile. Allo stesso modo, un ente potrebbe continuare a usare e mantenere una parte di software obsoleti ben oltre il tempo in cui le altre istituzioni hanno già eseguito aggiornamenti delle nuove versioni. Perciò, probabilmente risulta più utile parlare di 'obsolescenza istituzionale', cioè la tecnologia in questione non viene più utilizzata o un ente in particolare non può facilmente accedervi.

L'obsolescenza rappresenta un problema perché tutti i file presentano proprie dipendenze hardware e software. Questo si verificava soprattutto agli inizi della diffusione dell'informatica. II cambiamento rappresenta un problema quando compromette il significato del contenuto o della sua interpretazione da parte di un utente. L'obiettivo principale delle azioni di conservazione digitale è quello di preservare l'integrità e l'autenticità del materiale che si sta conservando, a dispetto di questi cambiamenti generazionali nella tecnologia informatica. Nella prossima sezione ci occuperemo delle strategie comuni usate per minimizzare questi cambiamenti.

\section{Strategie di conservazione}

In questa sezione si riportano le strategie tecniche che possono essere usate per la conservazione dell'informazione digitale. Verso la fine degli anni '90 si assiste a dei progressi minimi nell'individuazione di nuove strategie, sebbene ci siano state importanti ricerche così come uno sviluppo di diverse opzioni d'implementazione e di supporto tecnologico, tra cui la garanzia di qualità, informatica forense, registry delle rappresentazioni tecniche delle informazioni. Le tecniche qui presentate sono le seguenti:

- Migrazione dei formati

- Emulazione

- Musei informatici

\section{Migrazione dei formati}

La migrazione dei formati è una delle strategie di conservazione maggiormente utilizzate e gran parte dei sistemi di conservazione digitale contiene dei dati relativi alla funzionalità e al sistema dati che prevede una soluzione di migrazione. La migrazione dei formati è diversa dalla migrazione dei supporti di archiviazione: comprende, infatti, il passaggio o la trasformazione (ovvero, migrazione) dei dati da un formato datato/obsoleto in uno nuovo, possibilmente utilizzando per ogni passaggio nuovi sistemi applicativi per interpretare le informazioni. Passare da una versione di un formato standard a un altro successivo rappresenta una tipologia di questo metodo; per esempio, passare dalla versione 6 di MS Word (dal 1993) a MS Word per Windows 2010. Per le strutture e gli strumenti considerati utili per la valutazione dell'obsolescenza tecnica dei formati dei file si veda Formati dei file e standard.

La migrazione dei formati, come ogni intervento che è in grado di poter cambiare la struttura e il contenuto dei dati, può comportare alcuni errori e una perdita delle informazioni. Perciò, è 
importante stabilire delle metriche per calcolare la possibile perdita di informazioni e utilizzarle per fare delle verifiche sulla correttezza e qualità della migrazione dei formati.

Lavori recenti sulla garanzia di qualità e sulle azioni di conservazione digitale sono quelli di AQUA, SPRUCE e i progetti SCAPE. Per calcolare i livelli di errore, è necessario stabilire metriche molto specifiche. È probabile che si debba valutare cosa si considera errore e quale sia più importante rispetto ad altri. Ciò dipende dal contesto/contenuto del registro e da quali caratteristiche della risorsa vengono considerate come talmente 'significative' da essere conservate, così come dagli strumenti di migrazione e successivi formati che vengono impiegati in un percorso di migrazione.

Alcuni aspetti pratici coinvolti in questo processo comprendono la tempistica migliore per iniziare la migrazione - è meglio farlo da generazione a generazione, o dovrebbe essere saltata qualche generazione? Si dovrebbe tenere traccia di tutte le trasformazioni, dei loro risultati e documentare le perdite rilevate di informazione in modo da mantenere evidenze di autenticità e autorevolezza. PREMIS può essere uno strumento utile per questo scopo - per ulteriori informazioni su questo standard, si veda la sezione del Manuale Metadati e documentazione. È sempre buona prassi mantenere il formato del file originale così come depositato per restituirlo qualora necessario.

\section{Emulazione}

L'emulazione rappresenta una soluzione alternativa alla migrazione che permette agli archivi di conservare e fornire l'accesso agli utenti direttamente a partire dai file originali. Questa tecnica cerca di conservare i comportamenti originari, le caratteristiche delle applicazioni, così come il contenuto informativo. Si basa sul fatto che solo il programma originario è considerato come autorità competente sul formato e ciò risulta particolarmente utile per oggetti complessi caratterizzati da molteplici interdipendenze, come ad esempio i giochi o le app interattive.

Un emulatore, come si evince dal nome stesso, è un programma che gira su un'architettura informatica esistente, ma che offre gli stessi servizi e prestazioni di una precedente. Questo approccio è stato adottato da una serie di organizzazioni di beni culturali, spesso in collaborazione con esperti tecnici, e negli ultimi anni ha avuto un notevole successo nell'implementazione di soluzioni emulative per il patrimonio culturale (si veda Risorse). Tuttavia, permangono alcune problematiche, non da meno spesso quelle di diritti associati a licenze software che devono essere risolte (Rosenthal 2015b).

Un vantaggio particolare garantito dal processo di emulazione è che una singola soluzione può essere dispiegata su diversi oggetti, fino al punto in cui quegli oggetti sono usufruibili sullo stesso sistema operativo o hardware stack. L'uso di attrezzature informatiche obsolete potrebbe comunque risultare difficile per gli utenti, sebbene grazie ad esse possano accedere quasi certamente ad un'"autentica" rappresentazione dei registri. Di certo gli emulatori devono essere realizzati e manutenuti, richiedendo un insieme di competenze da rendere disponibili, aspetto 
non scontato. Si avrà bisogno di nuovi emulatori considerando che le architetture informatiche vanno incontro a obsolescenza, entrambe le cose comportano dei costi e necessità di risorse.

\section{Musei informatici}

Questa metodologia propone la conservazione dei computer e dei loro sistemi software (sistemi operativi, driver, ecc.) così come dei dati e dei programmi applicativi. Bisogna impegnarsi per mantenere in buono stato tutte le piattaforme e per conservare tutta la conoscenza necessaria per il mantenimento e l'utilizzo dei sistemi e dei loro programmi. L'idea è quella di avere anche delle soluzioni alternative destinate a diminuire così come l'insieme delle competenze. Dunque, questa strategia tende ad essere una misura provvisoria piuttosto che una soluzione a lungo termine. Esistono alcuni musei formali come il Computer History Museum in California e il Centre for Computing History a Cambridge. Generalmente questi ultimi mantengono i sistemi correttamente funzionanti anche non fornendo dei servizi di conservazione. Per ulteriori informazioni sui vecchi formati dei file e media si veda la sezione del Manuale Supporti obsoleti.

\section{Implementazione}

I DPC Technology Watch Reports sono una guida particolarmente utile per i generi e formati dei file più noti (tra cui email, social media, media Audio-Visivi, eBooks, e-Journals, GIS, CAD, archiviazione web, ecc.) e mostrano quali strategie tendono ad essere usate più comunemente in ognuna di queste aree. Gli strumenti che supportano le strategie di implementazione e conservazione sono presentati nella sezione generale del Manuale Soluzioni tecniche e $\underline{\text { strumenti, in particolare nella sezione Formati dei file e standard. }}$

\section{Risorse}

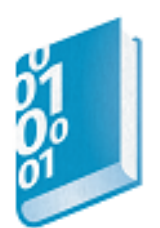

\section{DPC Technology Watch Report series}

http://www.dpconline.org/publications/technology-watch-reports

Le serie DPC Technology Watch Report vuole essere un'introduzione avanzata su aspetti specifici per chi ha il compito di definire e implementare servizi per l'accesso a lungo termine. Identificano e tengono traccia degli sviluppi nel campo IT, negli standard e strumenti che sono essenziali per le attività di conservazione digitale. Sono commissionati da esperti su questi sviluppi, e, prima della loro pubblicazione, vengono esaminati nella loro interezza da colleghi.

\section{Emulation \& Virtualization as Preservation Strategies}

https://mellon.org/media/filer public/0c/3e/0c3eee7d-4166-4ba6-a767-

\section{6b42e6a1c2a7/rosenthal-emulation-2015.pdf}

Questo report del 2015 sull'Emulation and Virtualization as Preservation Strategies di David Rosenthal è stato finanziato dalla Mellon Foundation, dalla Sloan Foundation e IMLS. Riflette su come gli sviluppi attuali sui sistemi di emulazione abbiano reso possibile la realizzazione delle emulazioni ai lettori mediante il Web in modo che le facciano come elementi normali delle 
pagine Web. Ciò comporta l'eliminazione di ciò che prima rappresentava il limite principale allo sviluppo dell'emulazione come strategia di conservazione. Gli ostacoli rimangono, i due più importanti sono relativi al fatto che gli strumenti per la creazione di immagini di sistema conservate sono inadatti e che il fondamento giuridico per la realizzazione delle emulazioni non è chiaro, e quando lo è, è molto restrittivo. Ciascuno di questi ostacoli aumenta i costi per la realizzazione e la garanzia d'accesso a collezioni consistenti e ben curate di artefatti digitali emulati fuori portata. Qualora venissero superati questi limiti, nei prossimi anni l'emulazione giocherà un ruolo molto più importante nella conservazione digitale (37 pagine).

Systematic planning for digital preservation: evaluating potential strategies and building preservation plans

http://www.ifs.tuwien.ac.at/ becker/pubs/becker-ijdl2009.pdf

Questo articolo pubblicato nel 2009 descrive un approccio sistematico per la valutazione di potenziali alternative alle attività di conservazione e per la costruzione di piani di conservazione accurati e affidabili per il mantenimento nel tempo dei contenuti digitali. II lavoro fu intrapreso come parte del progetto PLANETS finanziato dall'UE. (25 pagine).

\section{File format conversion}

http://www.nationalarchives.gov.uk/documents/information-management/format-

\section{conversion.pdf}

La conversione dei formati potrebbe aiutare a mantenere l'accesso, a usare le proprie informazioni e a mitigare i rischi portati dall'obsolescenza. Questa guida del 2011 del National Archives presenta i passaggi da compiere nell'esecuzione del processo di conversione dei formati. (29 pagine)

\section{What organizations are preserving software}

http://qanda.digipres.org/1068/what-organizations-are-preserving-software

Questo post, insieme alle risposte, di agosto del 2015, sulla pagina delle Q\&A del sito della Digital Preservation offre una lista utile e dei link che rimandano agli istituti che lavorano per la conservazione dei software per le strategie di emulazione.

\section{SCAPE Project Final best practice guidelines and recommendations}

http://scape-project.eu/wp-content/uploads/2014/02/SCAPE D20.6 KB V1.0.pdf

Questo report del progetto SCAPE pubblicato nel 2014 riguarda tre aree principali: implementazione del processo di migrazione su larga scala come strategia di conservazione; conservazione dei dati della ricerca; conservazione dei Bit. (127 pagine). 
The Internet Arcade

https://archive.org/details/internetarcade

L'Internet Arcade è una biblioteca web di videogame arcade (a gettoni) dagli anni '70 fino agli anni '90 dell'Internet Archive, implementata usando una soluzione di emulazione in-browser per garantire l'accesso alla collezione.

\section{Rhizome}

\section{https://sites.rhizome.org/theresa-duncan-cdroms/}

Negli anni '90, Theresa Duncan e collaboratori realizzarono tre videogame che fornivano un esempio di storytelling interattivo nella sua versione migliore. Due decenni più tardi, questi lavori vennero dimenticati (come la maggior parte dei CD-rom). Questa rassegna online, copresentata da Rhizome e il New Museum, li riporta in mostra, e permette di giocare online attraverso l'emulazione.

\section{Assessing Migration Risk for Scientific Data Formats}

http://www.ijdc.net/index.php/ijdc/article/view/202/271

Questo articolo si basa su una semplice ipotesi: laddove esistano percorsi di migrazione, la maggior parte dei file di dati scientifici possono essere migrati in sicurezza, lasciando solo una parte da gestire in modo più accurato, questo nel contesto di diversi formati di dati scientifici che sono o sono stati ampiamente utilizzati. L'approccio si basa sulla raccolta di informazioni sulle potenziali discrepanze osservate nella migrazione e, con l'uso di strumenti personalizzati, sulla valutazione di una consistente collezione di file dati nell'eventualità che si verificano tali rischi. I risultati supportano l'ipotesi di partenza, ma con alcuni avvertimenti.

\section{Portico - Preservation Step-by-Step}

https://www.portico.org/our-work/preservation-step-step/

Una guida passo dopo passo utile per la pianificazione di conservazione e strategie di migrazione utilizzate da Portico. Il piano di conservazione potrebbe comprendere una migrazione iniziale del packaging o file in formati specifici (per esempio, Portico migra gli articoli in formato XML di riviste elettroniche specifiche verso lo standard archivistico NLM).

Trash to treasure: Retro computer, software collection helps National Library access digital pieces

http://www.abc.net.au/news/2015-06-20/collecting-retro-computer-technology-to-save-digitaltreasures/6560494

La National Library australiana rese pubblico il suo impegno per lo sviluppo di una collezione di hardware e software informatici obsoleti. La usa per supportare il recupero dei dati e 
successivamente adotta altre strategie di conservazione, e non si affida al museo informatico per la conservazione a lungo termine.

\section{Riferimenti}

David Rosenthal, 2015a. "The Prostate Cancer of Preservation" Re-examined. Disponibile al seguente link: http://blog.dshr.org/2015/09/the-prostate-cancer-of-preservation-re.html David Rosenthal, 2015b. Emulation \& Virtualization as Preservation Strategies. Disponibile al seguente link: https://mellon.org/media/filer public/0c/3e/0c3eee7d-4166-4ba6-a7676b42e6a1c2a7/rosenthal-emulation-2015.pdf

Andrew N. Jackson, 2012. Formats over Time: Exploring UK Web History. Disponibile al seguente link: http://arxiv.org/abs/1210.1714 


\section{Accesso}

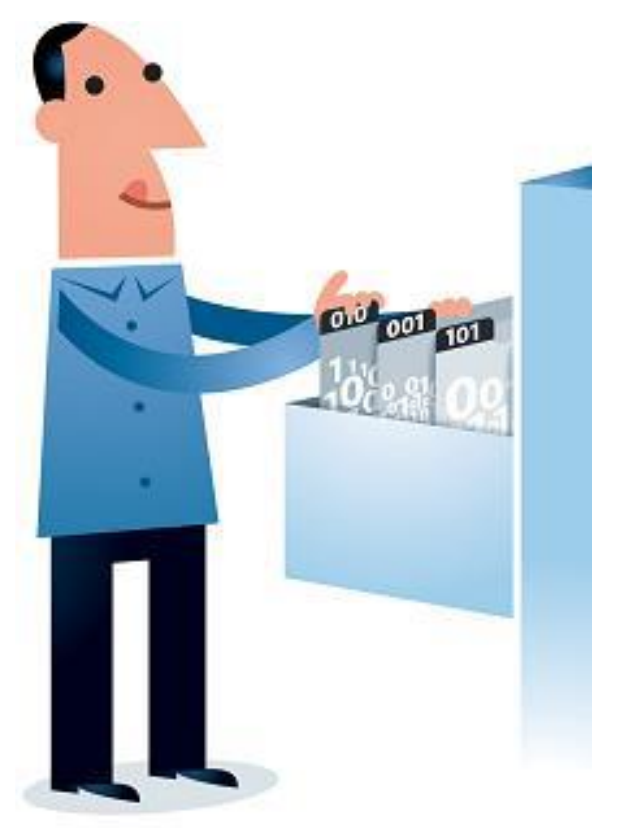

Illustrazione di Jørgen Stamp digitalbevaring.dk CC BY 2.5 Denmark

\section{Introduzione}

Da sempre esiste un forte legame tra conservazione e accesso. L'obiettivo principale della conservazione di contenuti informativi delle risorse tradizionali è fare in modo che esse rimangano accessibili per le generazioni sia presenti che future. Mantenere l'accesso agli oggetti digitali è un obiettivo importante dei programmi di conservazione digitale, ma è necessario che vi sia una gestione più attiva per tutto il ciclo di vita della risorsa prima che possa essere garantita. Perciò, risulta fondamentale tenere in considerazione questioni importanti per fornire l'accesso sin dall'inizio del processo di conservazione, l'ideale sarebbe già dalla fase di acquisizione. Se ne può trovare una descrizione nella sezione di questo Manuale Alberi decisionali per la selezione delle risorse digitali per la conservazione a lungo termine, che è inclusa all'interno dell'altra sezione Acquisizione e valutazione. In quest'ottica, questa sezione ha lo scopo di individuare gli aspetti principali da dover considerare, le decisioni che dovrebbero essere prese quando si pianifica di offrire l'accesso e come queste ultime possano influire sulla conservazione da un punto di vista più generale.

\section{Capire gli utenti}

Quando si pianifica di garantire l'accesso agli oggetti digitali è fondamentale capire chi sono i possibili utenti, così come lo è anche nelle attività più generali di conservazione digitale. L'importanza di un lavoro del genere risulta forse più evidente nell'interesse verso le 'Comunità designate' all'interno del modello Open Archival Information System Reference Model. Le conoscenze acquisite riguardo a questi utenti potenziali forniranno informazioni sulle decisioni prese per tutto il ciclo di vita, ma avranno probabilmente più valore nella scelta di soluzioni di erogazione di accesso appropriate controbilanciate con le risorse e con una serie di 
considerazioni tecnologiche. $\grave{E}$ anche importante adottare un approccio che porti all'individuazione delle comunità di utenti e delle loro esigenze in modo sistematico e oggettivo. In sintesi, comprendere cosa vogliono gli utenti e quale funzionalità può essere garantita dal repository.

La metodologia utilizzata per la raccolta di queste informazioni varierà a seconda del contesto organizzativo. Tra le possibili alternative e strumenti ci sono:

- L'analisi dell'utilizzo corrente (richieste di accesso per gli oggetti sia fisici che digitali, statistiche web, ecc.)

- Indagini

- Focus groups

- Interviste

- Casi di studio

- Analisi delle attività

Quando si avvia un'analisi degli utenti è importante prendere in considerazione sia chi sono e chi non sono gli utenti. Sebbene l'interazione con questi ultimi sia intrinsecamente più difficile, questo processo potrebbe essere utile per comprendere gli ostacoli esistenti da sfruttare così come per identificare nuovi possibili settori di mercato.

Una volta raccolte, queste informazioni dovrebbero essere impiegate per comunicare le decisioni che sono state prese in riferimento all'implementazione delle soluzioni di erogazione di accesso. Risulta anche importante continuare a monitorare lo sviluppo delle comunità di utenti e ciò dovrebbe venire incorporato nelle attività standard del Piano di conservazione all'interno della propria organizzazione.

\section{Formati di accesso}

Un aspetto significativo nel momento in cui si pianifica l'accesso è il formato con cui gli oggetti digitali verranno resi disponibili agli utenti. Se da una parte esiste un forte legame tra la conservazione e l'accesso in termini di obiettivi prioritari connessi al programma di conservazione digitale, dall'altra si deve fare una chiara distinzione tra questi ultimi. Vi possono essere diverse ragioni tecniche, legali e pragmatiche per separare la copia d'accesso da quella di conservazione, perciò potrebbe essere auspicabile, o perfino necessario, realizzare una copia di accesso dell'oggetto digitale per l'utente in un formato differente da quello usato nell'archiviazione di sistema della conservazione. Infatti, un'organizzazione può voler offrire diversi 'stili' di formato a seconda delle necessità di un particolare utente o della comunità in questione. Quando si selezionano i formati per l'accesso, un'organizzazione deve tenere in conto una serie di aspetti, tra cui:

- Quale è il formato più comunemente/ampiamente supportato per il tipo di oggetto?

- Gli utenti avranno accesso a visualizzatori gratuiti/software che supportano il tipo di file proposto?

- Quale dimensione del file viene prodotta e quali sono le ripercussioni connesse all'erogazione per l'utente? 
- Il formato è facile da usare?

- Gli utenti avranno bisogno di una guida o di una documentazione di supporto perché abbiano accesso/usino gli oggetti?

- L'organizzazione ha delle comunità di utenti distinte con requisiti di accesso differenti?

Per dei dettagli sui formati comuni di conservazione e accesso, si veda anche la sezione Formati dei file e standard.

\section{Aspetti legali riferiti all'accesso}

Ci sono diversi aspetti legali di cui ci si dovrà probabilmente occupare quando si garantisce l'accesso agli oggetti digitali, che riguarderanno sia le soluzioni tecnologiche che vengono impiegate, sia chi e quando può accedere alle risorse. Queste, come precedentemente menzionato, sono delle considerazioni importanti che si vanno a sovrapporre alle acquisizioni ed è importante che le informazioni corrette vengano raccolte in un dato momento per facilitare le richieste d'accesso che si presenteranno successivamente nel ciclo di vita. Senza queste informazioni potrebbe non essere possibile gestire in modo adatto l'accesso e si potrebbe esporre l'organizzazione a una serie di possibili rischi legali.

Gli aspetti legali da dover tenere in conto comprenderanno:

- Restrizioni d'uso riferite alla sensibilità e protezione dei dati

- Divieti concordati sul contenuto laddove un accesso iniziale potrebbe rappresentare una violazione del contratto

- Gestione dei diritti di proprietà intellettuale, ad es., copyright

In particolare, la gestione dell'IPR dovrebbe essere allineata al processo di acquisizione considerando attentamente gli accordi di trasferimento e di proprietà e le licenze di copyright messe in atto in quel momento. Le licenze devono chiaramente dichiarare l'accesso autorizzato e riutilizzare le autorizzazioni e procedure per l'accesso, che sia gestito attraverso un sistema di gestione di diritti o altre modalità.

\section{Forme di fornitura di accesso}

Le ultime importanti decisioni che un'organizzazione deve prendere riguardano:

- Politiche

- Procedure

- Servizi gratuiti o a pagamento

- Accesso online/offline e il contesto d'accesso offerto

- Archiviazione e sicurezza

Se la copia di accesso è l'unica copia di una risorsa digitale, allora il pericolo di perderla a causa di furti o danni risulta chiaramente alto. Se viene adottato questo approccio, si deve avviare una valutazione del rischio che comprende alcune delle seguenti questioni (si vedano anche Acquisizione e valutazione e Archiviazione): 


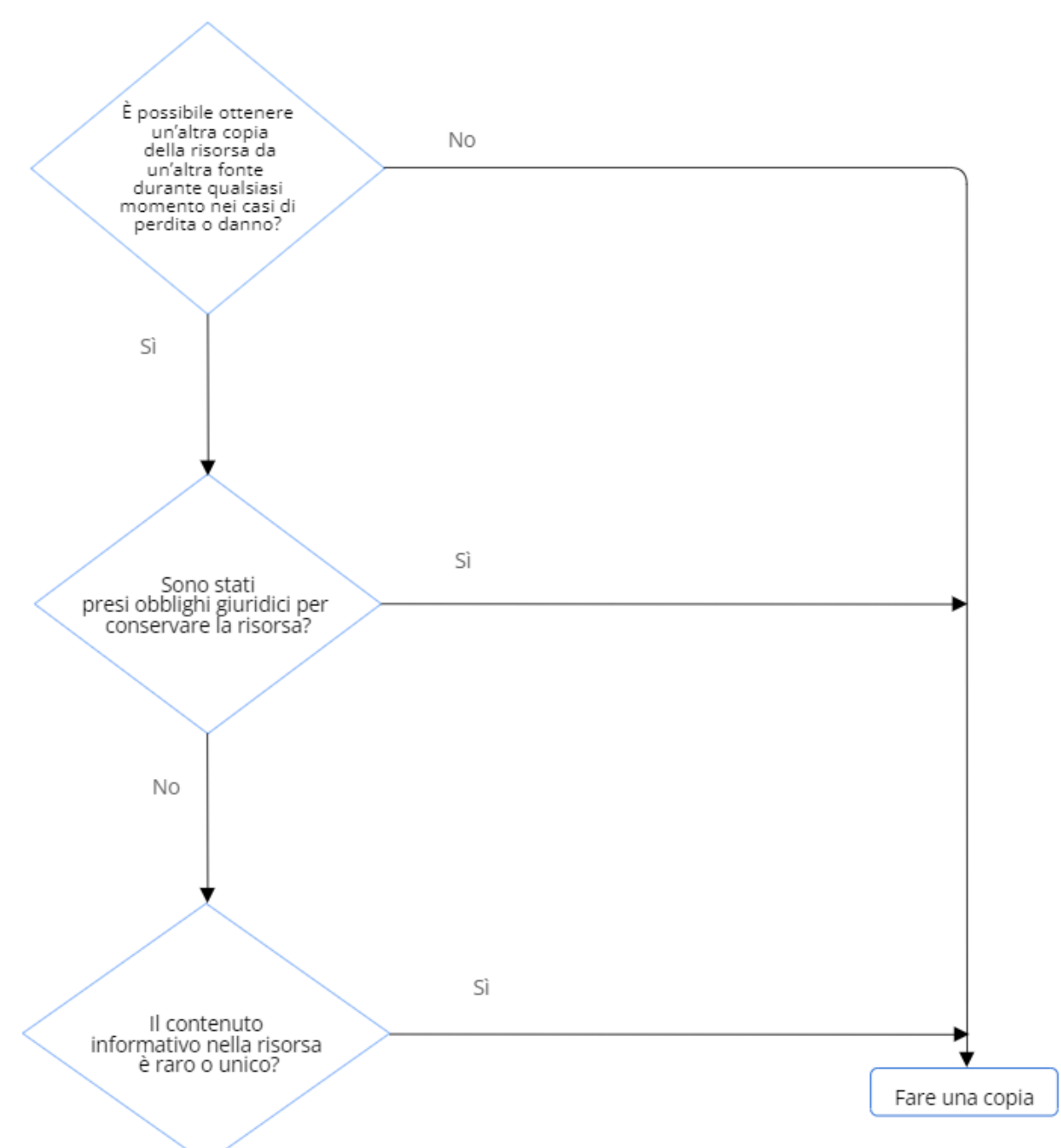

\section{Conclusioni}

L'accesso è strettamente legato a molti altri aspetti della conservazione digitale e delle tecnologie trattate nel Manuale. In particolare, potreste voler visitare le sezioni Strategie e politiche istituzionali, Conformità giuridica, Metadati e documentazione, Acquisizione e valutazione, Archiviazione, Supporti obsoleti, Formati dei file e standard, e Sicurezza delle informazioni. 


\section{Risorse

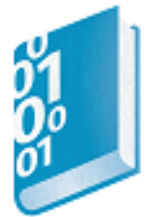

Born-Digital Access in Archival Repositories: Mapping the Current Landscape, Preliminary Report August 2015

https://docs.google.com/document/d/15v3Z6fFNydrXcGfGWXA4xzyWlivirfUXhHoggVDBtUg/pr eview?sle=true\#

Questo interessante documento rappresenta i risultati preliminari e l'analisi di uno studio sulle attuali pratiche di accesso native digitali su oltre 200 istituti di patrimonio culturale. I partecipanti provengono principalmente dagli USA.

Reference model for an open archival information system (OAIS): Recommended practice (CCSDS 650.0-M-2: Magenta Book), Consultative Committee for Space Data Systems 2012

https://public.ccsds.org/pubs/650x0m2.pdf

Pubblicato successivamente come ISO 14721:2012, Space Data and Information Transfer Systems - Open Archival Information System (OAIS) - Reference Model, 2nd edition. La funzione d'Accesso all'interno di OAIS gestisce i processi e i servizi per cui gli utenti - e in particolare la Comunità Designata - collocano, richiedono e ricevono i dati che si trovano all'interno dell'archiviazione dei dati OAIS. Pertanto, è il meccanismo principale tramite il quale l'archivio assolve alla sua responsabilità di rendere le sue informazioni accessibili alla sua comunità di utenti. (135 pagine).

Adrian Brown 2013 Practical Digital Preservation a how-to guide for organizations of any size

II Capitolo 9 (28 pagine) di questo libro è dedicato alla tematica relativa all'accesso da garantire agli utenti.



\section{Community Owned digital Preservation Tools Registry COPTR}

http://www. digipres.org/tools/

Esistono diversi strumenti per l'accesso e altri che presentano al loro interno la funzionalità d'accesso. II Manuale consiglia di fare una ricerca mediante il tool POWRR Grid che si trova all'interno di COPTR. POWRR Grid fornisce un set di visualizzazioni interattive progettate per supportare i professionisti nell'identificazione e selezione di strumenti di cui hanno bisogno per affrontare le sfide legate alla conservazione digitale. La colonna dell'Accesso, Utilizzo e RiUtilizzo della griglia identifica gli strumenti di accesso per tipi specifici di contenuti o gli strumenti generici e i sistemi che presentano funzioni di accesso. Tutto quello che si trova nella griglia ha 
dei collegamenti ipertestuali, per cui, con un semplice click sul display si possono trovare le informazioni richieste. Cliccando sul nome di uno specifico tool per la conservazione sarà possibile ottenere più dettagli sul wiki COPTR, posizione suggerita per estendere o aggiornare le informazioni sugli strumenti.

AIMS Born-Digital Collections: An Inter-Institutional Model for Stewardship. University of Hull, Stanford University, University of Virginia, and Yale University (2012)

http://dcs.library.virginia.edu/files/2013/02/AIMS final.pdf

II sistema AIMS (An Inter-Institutional Model for Stewardship) è una metodologia di gestione delle risorse native digitali. È diviso in quattro sezioni principali per le procedure di alto livello orientate a flussi di lavoro nativi digitali: sviluppo della collezione, selezione, conservazione e descrizione, rilevamento e accesso. L'accesso riguarda principalmente la revisione e le informazioni riservate. Le appendici comprendono, ad esempio, alcuni casi di diagrammi di elaborazione di workflow, un'analisi degli strumenti, un'indagine sui donatori. (195 pagine).

\section{Casi di studio}

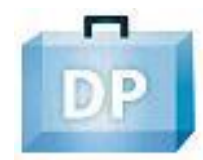

TNA case studies: Online access

http://www.nationalarchives.gov.uk/archives-sector/online-access.htm

Una serie di nove casi di studio pubblicati da TNA su come le collezioni siano state rese più accessibili inserendo i record online. Sono tratti da diversi archivi.

\section{Codebreakers: makers of modern genetics}

https://digirati.com/work/galleries-libraries-archives-museums/case-studies/wellcome-library/

Un caso di studio di DIGIRATI, gli sviluppatori del lettore della Wellcome Trust Library, riferito al lettore in uso nel momento in cui si accede alla collezione Francis Crick. Il lettore digitale della Wellcome Library è per tutti gratuitamente accessibile per il download e per l'utilizzo, può essere usato per visualizzare tutti i tipi di contenuto digitale, compresi i libri nella loro interezza, archivi, opere d'arte, file video e file audio. II software può essere scaricato dall'account GitHub della Wellcome Library (https://github.com/wellcomelibrary/player).

\section{Managing Risk with a Virtual Reading Room: Two Born Digital Projects, Michelle Light http://digitalscholarship.unlv.edu/cgi/viewcontent.cgi?article=1462\&context=lib articles}

Tra il 2010 e il 2013, Irvine, I'Università della California, ha lanciato un sito per fornire un accesso online agli articoli personali di Richard Rorty e Mark Poster sotto forma di una stanza virtuale di lettura. Quest'ultima, avendo posto un limite al numero di utenti qualificati e alla reperibilità del testo completo sul web aperto, ha ridotto i rischi associati a questo tipo di accesso a risorse personali e di archivio per quanto riguarda gli aspetti riferiti alla privacy e al copyright. II caso di studio analizza ogni fase della ricerca e dell'idea, compresi i progetti comparativi che avvengono presso altri enti e le lezioni apprese, in una maniera molto aperta e informativa. 
From Accession to Access: A Born-Digital Materials Case Study, by Cyndi Shein Journal of Western Archives Volume 5 Issue 1 (2014): 1-42

http://digitalcommons. usu.edu/cgi/viewcontent.cgi?article=1036\&context=westernarchives

Tra il 2011 e il 2013 il Getty Institutional Records and Archives portò a termine la sua prima iniziativa di ingest completo, nella messa a disposizione, descrizione e rilascio di risorse native digitali uniche nel momento di ricezione di interviste di storia orale realizzate da alcuni partner del progetto Pacific Standard Time: Art a Los Angeles. Questo caso di studio riguarda le sfide e le possibilità relative a questa collezione ibrida di registrazioni audiovisive, file digitali multimediali, e trascrizioni analogiche. Descrive gli sforzi degli Archivi nello sviluppo di un workflow di elaborazione di base che applichi la strategia di gestione della risorsa, comunemente chiamata 'MPLP', in un ambiente digitale, cercando di salvaguardare l'integrità e l'autenticità dei file, di essere in linea con gli standard professionali e di rispettare i principi archivistici fondamentali. Lo studio descrive il workflow ottenuto e mette in evidenza alcune delle tecnologie poco costose che vennero impiegate con successo per automatizzare o accelerare i passaggi necessari all'elaborazione del contenuto trasferito attraverso supporti facilmente accessibili e formati dei file vigenti. 


\section{Metadati e documentazione}

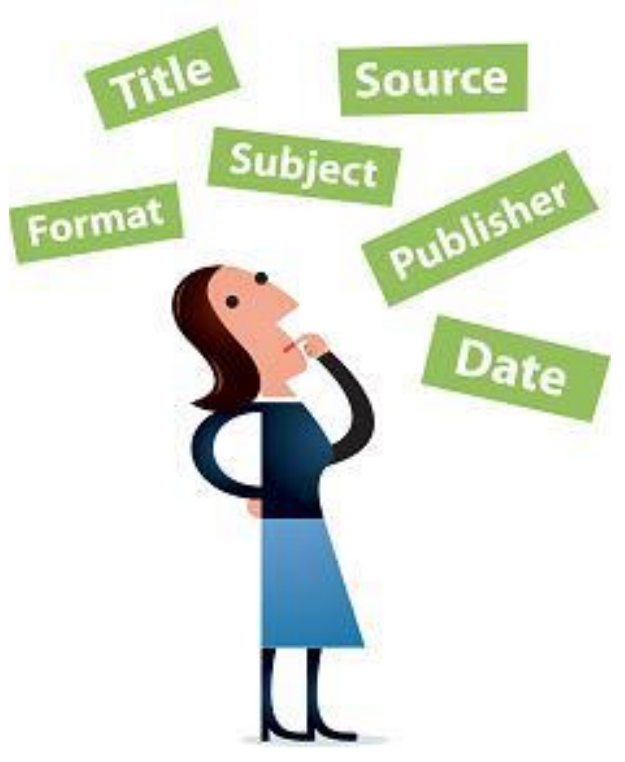

Illustrazione di Jørgen Stamp digitalbevaring.dk CC BY 2.5 Denmark

\section{Introduzione}

Questa sezione offre una breve panoramica per utenti principianti e di livello intermedio riguardo ai metadati e alla documentazione, con un focus sullo standard di standard di metadati per la conservazione digitale PREMIS. Attinge alla seconda edizione del DPC Technology Watch Report sui Metadati di Conservazione. II report tratta una serie di aspetti su ampia scala e di attività in modo più approfondito con ulteriori letture e suggerimenti (Gartner and Lavoie, 2013). Viene indicata ai lettori che hanno bisogno di un livello di informazioni più avanzato.

I metadati sono dei dati di una risorsa digitale che vengono archiviati in una forma adatta al processamento informatico. Sono utili per molti degli obiettivi della conservazione a lungo termine, fornendo un registro delle attività che sono state eseguite sulle risorse digitali e una base su cui poter prendere, in prospettiva, decisioni future sulle attività di conservazione, così come supportando delle scoperte e utilizzi. Le informazioni che sono contenute all'interno di un record di metadati spesso contemplano una serie di argomenti. Non esiste una linea netta tra ciò che sono i metadati di conservazione e ciò che non sono, ma fondamentalmente l'obiettivo dei metadati di conservazione è di supportare gli scopi della conservazione digitale a lungo termine, che sono quelli relativi al mantenimento della disponibilità, identità, persistenza, resa, comprensibilità, e autenticità degli oggetti digitali nei lunghi periodi.

La documentazione è l'informazione (come i manuali dei software, schemi di indagini, o guide per gli utenti) offerta dal creatore e dal repository che completa i metadati e fornisce informazioni sufficienti per far sì che la risorsa possa essere usata da altri. Spesso è l'unico mezzoche offre una panoramica su come una risorsa digitale è stata creata, manipolata, gestita e utilizzata dal 
suo creatore, e, solitamente, è da qui che gli altri comprendono la modalità d'uso appropriata per la risorsa.

Diversi fattori rendono i metadati e la documentazione particolarmente critici per la continuità delle risorse digitali e si riferiscono a differenze sostanziali tra le risorse tradizionali e digitali:

- Tecnologia. Perché siano comprensibili, le risorse digitali dipendono da sistemi hardware e software. I requisiti tecnici devono essere registrati in modo che vengano prese decisioni appropriate su strategie di conservazione e di accesso.

- Cambiamento. Mentre le risorse tradizionali potrebbero venir conservate da programmi preventivi di conservazione prevalentemente passivi, le risorse digitali saranno sottoposte ad azioni ripetute, e ci saranno molti operatori e possibilmente anche diverse istituzioni che influiranno sulla gestione delle risorse digitali su un periodo di tempo prolungato. La registrazione delle azioni intraprese su una risorsa e i cambiamenti che si verificano come conseguenza saranno essenziali per i futuri gestori e utenti della risorsa.

- Autenticità. I metadati e la documentazione potrebbero essere i principali, se non del tutto gli unici, mezzi per definire in modo affidabile l'autenticità delle risorse seguendo i cambiamenti.

- Gestione dei diritti. Mentre le risorse tradizionali potrebbero o meno essere copiate come parte del programma della loro conservazione, quelle digitali devono essere copiate se devono rimanere accessibili. I gestori devono sapere se hanno il diritto a copiare ai fini della conservazione, quali (se ci sono) tecnologie sono state usate per controllare la gestione dei diritti e quali (se ci sono) ripercussioni esistono per il controllo dell'accesso.

- Riutilizzi futuri. Potrebbe non essere possibile per gli utenti usare le risorse senza una documentazione appropriata.

- Costi. Creare manualmente i metadati è costoso e i metadati di conservazione potrebbero non sempre essere facilmente realizzabili in modo automatico. I metadati aggiuntivi per esigenze di conservazione digitale richiedono quindi delle attente politiche di scambio costi/benefici.

\section{Lo standard PREMIS (PREservation Metadata: Implementation Strategies)}

PREMIS (PREservation Metadata: Implementation Strategies) è lo standard internazionale per i metadati che supporta la conservazione degli oggetti digitali e assicura il loro utilizzo a lungo termine. Sviluppato da un gruppo internazionale, PREMIS è adottato in progetti sulla conservazione digitale in tutto il mondo, e il suo supporto è integrato in una serie di sistemi e strumenti di conservazione digitale commerciale e open-source.

II PREMIS Data Dictionary (PREMIS, 2013) si organizza intorno a un modello di dati che è costituito da cinque entità associate al processo di conservazione digitale:

1. Entità intellettuale - un insieme omogeneo di contenuti descritto come un'unità: ad es., un libro 
2. Oggetto - un'unità di informazione discreta in forma digitale, ad es., un file PDF

3. Evento - un'azione di conservazione, ad es., ingest del file PDF nel repository

4. Agente - una persona, organizzazione, programma software associato a un Evento, ad es., l'editore di un file PDF

5. Diritti - una o più autorizzazioni che riguardano un Oggetto, ad es., l'autorizzazione a fare copie di un file PDF a fini della conservazione

Le unità semantiche definite nel PREMIS Data Dictionary rappresentano, prese insieme, l'informazione 'centrale' richiesta per supportare le attività di conservazione digitale nella maggior parte dei contesti di repository. Tuttavia, il concetto di 'centrale' riferito a PREMIS è definito vagamente: non tutte le unità semantiche sono considerate obbligatorie in tutte le circostanze, e alcune sono opzionali in tutte le situazioni. II Data Dictionary cerca di stabilire un equilibrio nel riconoscere che ci sarà una sovrapposizione importante nella necessità dei metadati nei diversi contesti di repository, e allo stesso tempo nel constatare che tutti i contesti in qualche modo sono diversi, per cui le loro rispettive necessità di metadati raramente saranno esattamente le stesse.

\section{Implementazione}

Anche se il PREMIS Data Dictionary non è uno standard formale, nel senso che non è gestito da un'agenzia di standard riconosciuta, è arrivato ad avere lo status di uno standard accettato per i metadati di conservazione nella comunità di conservazione digitale. Un punto di forza, ma che è anche un limite, del PREMIS Data Dictionary è che deve essere adattato ai requisiti di uno specifico contesto: non è una soluzione pronta all'uso, nel senso che un archivio non implementa semplicemente il Data Dictionary nella sua interezza. Solo una parte potrebbe essere rilevante per alcuni aspetti riferiti alla conservazione digitale; in alternativa, il repository potrebbe constatare che delle informazioni aggiuntive oltre a quello che viene definito nel Dizionario sono necessarie per sostenere le loro esigenze.

Per esempio, il Data Dictionary non presenta nessuna disposizione per la documentazione di informazioni sulle dipendenze aziendali o di policy di un repository, cosa che potrebbe essere richiesta per supportare il processo decisionale della conservazione.

In breve, ogni repository avrà bisogno di impegnarsi ad adattare i metadati di conservazione e gli standard di documentazione alle sue circostanze e necessità specifiche.

Durante l'implementazione, generalmente, un ente identifica il proprio standard di informazioni minimo necessario per i componenti catalogati nella collezione. Ogni ente può anche individuare i suoi livelli preferiti di metadati e di documentazione per le acquisizioni e potrebbe notificare e esortare $\mathrm{i}$ fornitori o i detentori a garantire queste informazioni. II personale revisiona e riconsidera le informazioni fornite per assicurarsi che queste siano conformi alle linee guida istituzionali e generino dei cataloghi di record per i dati depositati integrando la catalogazione e gli standard di documentazione per far sì che le informazioni su questi componenti siano rese disponibili agli utenti attraverso dei cataloghi appropriati. In molti casi le informazioni di contesto 
per le risorse saranno essenziali per il loro utilizzo futuro e questo aspetto della documentazione non dovrebbe essere trascurato.

Il livello di catalogazione e documentazione di accompagnamento viene aggiunto successivamente a un componente, e ogni limitazione che questi possono imporre può venire documentata a beneficio di utenti futuri. Laddove le risorse siano gestite da parti terze, ma rese disponibili mediante la figura di un'istituzione, le informazioni potrebbero essere fornite da parti terze in modalità concordate conformi alle linee guida istituzionali o nel formato nativo del fornitore.

Se c'è bisogno di un accesso avanzato, un ente può prendere l'iniziativa di potenziare le informazioni di documentazione e di catalogazione verso uno standard più specifico al fine di soddisfare le nuove esigenze. Una documentazione retrospettiva o un potenziamento del catalogo dovrebbero verificarsi anche quando la validazione o il controllo della documentazione e catalogazione di una risorsa dimostrano che si è andati sotto il minimo accettabile dello standard.

Un numero considerevole sia di utenti che di fornitori di metadati di conservazione ha adottato il PREMIS, e questi si sono occupati di molti degli ostacoli iniziali all'implementazione. II processo di implementazione di PREMIS in un contesto lavorativo è reso più facile da una serie di strumenti che estraggono i metadati dagli oggetti digitali e danno in output un XML PREMIS. La PREMIS Maintenance Activity tiene aggiornata una pagina web in cui elenca gli strumenti più importanti che possono essere utilizzati con PREMIS. Include anche una lista di discussione attiva per e-mail e un wiki per i documenti da condividere. Per ulteriori informazioni si veda la sezione seguente Risorse e casi di studio.

Si vedano anche le sezioni relative del Manuale che comprendono Acquisizione e valutazione, e Piano di conservazione.

\section{Risorse}

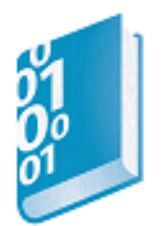

PREMIS Data Dictionary for Preservation Metadata, Version 3.0

http://www.loc.gov/standards/premis/v3/index.html

II Data Dictionary di PREMIS e la sua documentazione di supporto rappresentano una risorsa pratica e completa per l'implementazione dei metadati di conservazione nei sistemi di archiviazione digitale. II Data Dictionary è costruito sulla base di un modello dati che definisce cinque entità: Entità Intellettuali, Oggetti, Eventi, Diritti, e Agenti. Ogni unità semantica definita nel Data Dictionary è una proprietà di una delle entità nel modello dati. La versione 3.0 è stata pubblicata nel giugno del 2015 (273 pagine). 


\section{Preservation Metadata (2nd edition), DPC Technology Watch Report}

http://dx.doi.org/10.7207/twr13-03

Questo report si concentra sui nuovi progressi nei metadati di conservazione resi possibili dalla comparsa di PREMIS come uno standard internazionale de facto. Discute in particolare dei temi principali riferiti all'implementazione che comprendono le revisioni del Data Dictionary; sensibilizzazione della comunità; packaging (con un focus sui METS), strumenti, implementazioni di PREMIS nei sistemi di conservazione digitale e risorse applicative. Pubblicato nel 2013 (36 pagine).

\section{Tools for preservation metadata implementation}

http://www.loc.gov/standards/premis/tools for premis.php

II PREMIS Maintenance Activity gestisce una pagina web che elenca gli strumenti disponibili più importanti da usare con PREMIS. Ci sono riferimenti ad alcuni strumenti, insieme ad indicatori relativi ad altri che potrebbero essere utilizzati per la generazione dei file METS (Metadata Encoding and Transmission Standard - uno schema XML per il packaging dei metadati degli oggetti digitali) in combinazione con PREMIS. La maggior parte degli strumenti elencati serve per l'estrazione di metadati tecnici dagli oggetti digitali e per la loro conversione al fine di allinearli alla codifica dell'entità Oggetto di PREMIS. Altri possono essere utilizzati per controllare i formati o validare i file rispetto ai checksum.

WWW

\section{PREMIS website}

http://www.loc.gov/standards/premis/index.html

II Comitato Editoriale di PREMIS coordina le revisioni e l'implementazione dello standard PREMIS, che è costituito dal Data Dictionary, uno schema XML, e documentazione di supporto. II forum PREMIS Implementers' Group, all'interno dell'Attività di Mantenimento PREMIS, comprende un elenco di discussione e-mail attivo e un wiki per la condivisione dei documenti. II wiki è una risorsa particolarmente utile per i nuovi realizzatori in quanto comprende tutorial di PREMIS, una raccolta di esempi di utilizzo di PREMIS e link alle informazioni sugli strumenti di PREMIS. L'Attività di Mantenimento PREMIS gestisce un registry attivo delle implementazioni PREMIS.

\section{Documenting your data}

http://www.data-archive.ac.uk/create-manage/document

Un ottimo set di risorse di supporto per i ricercatori alla documentazione e metadatazione dei loro studi, elaborato insieme al Data Archive britannico.

\section{Archaeology Data Service Guidelines for Depositors}

http://archaeologydataservice.ac.uk/advice/guidelinesForDepositors 
Le Linee Guida ADS per i soggetti produttori offrono una guida su come pianificare in modo appropriato i dati e i metadati per il deposito con ADS. Vi è anche una serie di brevi schede sommarie e liste di controllo che riguardano: la gestione dei dati; la selezione e il mantenimento; i formati dei file prescelti e i metadati. Altre risorse comprendono una serie di Guide alle Buone Pratiche, che integrano le Linee Guida ADS e forniscono ulteriori informazioni sui tipi di dati specifici.

\section{Casi di studio}

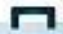

D)

DPC case note: British Library ASR2 using METS to keep data and metadata together for preservation

http://www.dpconline.org/component/docman/doc download/474-casenoteasr2.pdf

Questo caso di studio finanziato da JISC esamina il progetto 'Archival Sound Recordings 2' della British Library, specificando che una delle sfide relative all'accesso a lungo termine al contenuto digitalizzato è quella di garantire che l'informazione descrittiva e il contenuto digitalizzato non siano separati l'uno dall'altro. La British Library ha usato uno standard chiamato METS al fine di evitare questo rischio. Luglio 2010 (4 pagine).

\section{Designing Metadata for Long-Term Data Preservation: DataONE Case Study} https://doi.org/10.1002/meet.14504701435

Una breve descrizione di come PREMIS è stato utilizzato per definire i requisiti dei metadati di conservazione per i dati scientifici di DataONE (Data Observation Network for Earth). 2010 (2 pagine).

Preservica Case Study: Q\&A with Glen McAninch, Kentucky Department for Libraries and Archives

https://preservica.com/uploads/resources/Preservica-Kentucky-QA-2014 NEW.pdf

Glen McAninch discute dell'importanza della Provenienza, del Contesto e dei Metadati nella conservazione di documenti digitali.

\section{PREMIS Implementations Registry}

http://www.loc.gov/standards/premis/registry/index.php

II PREMIS Maintenance Activity consiste nella gestione di un registry attivo di oltre 40 implementazioni PREMIS con dettagli sul repository e sul suo uso di PREMIS. Nonostante non si tratti formalmente di casi di studio, le voci presentano dettagli sulle esperienze pratiche, ad esempio, Creare un repository digitale presso la Swedish National Archives usando PREMIS.

\section{Riferimenti}

Gartner, R. and Lavoie, B., 2013. Preservation Metadata (2nd edition), DPC Technology Watch Report 13-3 May 2013. Disponibile al seguente link: http://dx.doi.org/10.7207/twr13-03 
PREMIS, 2013. Data Dictionary for Preservation Metadata, Version 3.0. Disponibile al seguente link: http://www.loc.gov/standards/premis/v3/index.html 


\section{Soluzioni tecniche e strumenti}

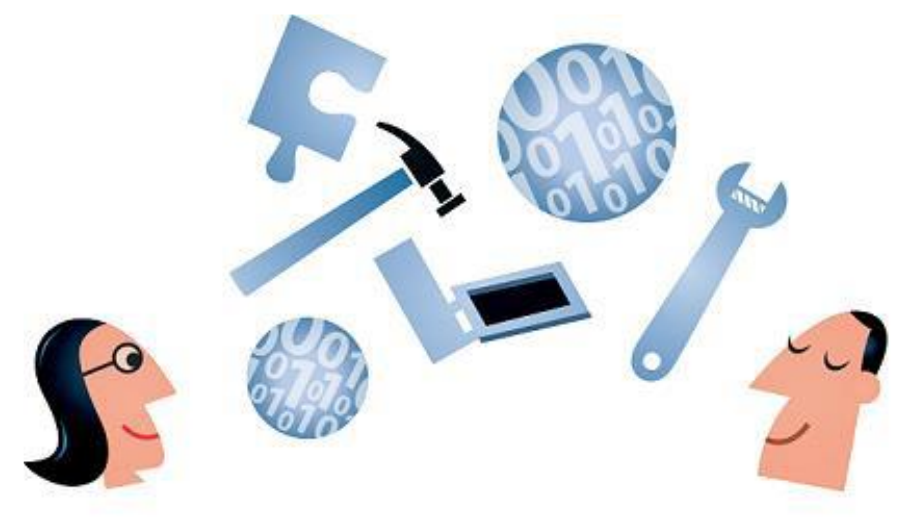

Illustrazione di Jørgen Stamp digitalbevaring.dk CC BY 2.5 Denmark

\section{A chi è rivolto?}

Responsabile dell'area operativa (DigCurV Manager Lens) e lo staff (DigCurV Practitioner Lens) addetti ai repository, editori e altri creatori di dati, fornitori di servizi terzi.

Requisiti minimi di conoscenza

Da principiante a intermedio.

\section{Obiettivo}

- Concentrarsi sugli strumenti tecnici e sulle applicazioni che supportano la conservazione digitale: software, applicazioni, programmi e servizi tecnici.

- Tener conto dell'implementazione pratica delle tecniche e delle tecnologie di conservazione sia sotto forma di programmi relativamente piccoli e discreti (come DROID) sia sotto forma di soluzioni a livello aziendale che integrano diversi strumenti.

- Questa sezione esclude altri aspetti più strategici e gli standard che spesso vengono descritti come strumenti: questi sono trattati in altre sezioni del Manuale.

\section{Riutilizzare queste informazioni}

È possibile riutilizzare questo materiale in lingua inglese (ad eccezione dei loghi) necessariamente con dei riconoscimenti gratuiti in qualsiasi formato o con qualsiasi mezzo. Per i dettagli completi sulle autorizzazioni e riconoscimenti per il riutilizzo si veda la sezione Come usare il Manuale.

Per autorizzazioni alla traduzione in altre lingue si contatti per e-mail:

handbook@dpconline.org

Si prega di usare questa citazione per il Manuale: Digital Preservation Handbook, 2nd Edition, https://www.dpconline.org/handbook, Digital Preservation Coalition (C) 2015. 


\section{Strumenti}

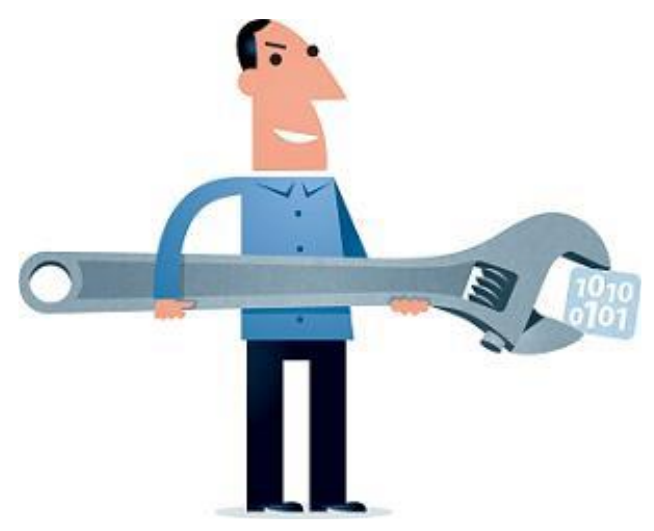

Illustrazione di Jørgen Stamp digitalbevaring.dk CC BY 2.5 Denmark

\section{Guida per principianti agli strumenti di conservazione digitale}

L'utilità degli strumenti tecnici per la conservazione digitale dipende dal contesto del loro impiego. Una raccomandazione da parte della comunità potrebbe essere significativa, ma se non si allinea con la propria specifica funzione o contesto organizzativo, c'è un'alta probabilità che lo strumento non funzioni (bene). Perciò, prima di selezionare gli strumenti di conservazione digitale, è importante tenere attentamente in considerazione il flusso di lavoro tecnico e l'assetto istituzionale in cui questi sono incorporati. Un esempio pratico di ciò è stato presentato da Northumberland Estates, che ha sviluppato un quadro di valutazione semplice per vagliare gli strumenti nel contesto.

Un modo alternativo di considerare questo argomento è quello di revisionare la portata entro cui un determinato strumento porta avanti azioni di conservazione che, a loro volta, derivano da un determinato quadro strategico.

\section{Riflettere sugli strumenti della conservazione digitale}

Solitamente, nel processo di implementazione degli strumenti di conservazione digitale si presentano le seguenti circostanze. Ciò che segue non è un elenco esaustivo, ma una riflessione su questi aspetti potrà aiutare a fare scelte ragionevoli e realistiche.

\section{Software open source versus software commerciali}

Alcune organizzazioni - spesso nei repository nel campo dell'istruzione superiore e in particolare in quelli della ricerca istituzionale - hanno familiarità con l'utilizzo di software open source, specialmente laddove dispongano di gruppi di sviluppatori interni. Per software 'open source'si intende che il codice sottostante viene reso gratuitamente disponibile, consentendo liberamente aggiunte, modifiche o sviluppi. Altre organizzazioni che non hanno un accesso facile agli sviluppatori, tendono a disporre di norme di acquisizione che preferiscono le soluzioni disponibili sul mercato coperte da contratti di assistenza continuativi. La distinzione tra software open 
source e software commerciali viene spesso enfatizzata perché entrambi si influenzano a vicenda. Tuttavia, nello scegliere gli strumenti si potrebbero dover considerare le norme e la cultura della propria organizzazione.

\section{Soluzioni a livello aziendale/d'impresa versus micro-servizi}

Alcuni strumenti di conservazione digitale sono progettati per offrire soluzioni dall'inizio alla fine (soup to nuts), cioè forniscono un processo integrato end-to-end che consente a tutte (o alla maggior parte) le funzioni di conservazione digitale di essere conseguite per l'intera organizzazione. Infatti, le soluzioni a livello aziendale spesso vengono realizzate aggregando singoli strumenti integrati in un'unica interfaccia. La soluzione per ogni problema specifico potrebbe essere relativamente semplice e l'organizzazione potrebbe essere felice di mettere insieme una serie di piccoli strumenti per funzionalità discrete. Ciò favorisce un rapido progresso ed è d'aiuto nel momento in cui si testano e sperimentano alcuni strumenti; ma può essere difficile da mantenere per un periodo di tempo prolungato. In altre organizzazioni esistono controlli molto più rigidi nell'impiego di software e si prevede che le soluzioni siano integrate in un intero flusso di lavoro, il che richiede soluzioni complete. L'inizio potrebbe essere più lento, ma può essere più sostenibile a lungo termine. Prima di selezionare uno strumento è utile considerare in che punto su questo spettro si posiziona la propria organizzazione.

\section{Descrivere i flussi di lavoro}

Una considerazione importante da fare per quel che riguarda gli strumenti è la loro posizione nel flusso di lavoro complessivo, perciò, prima di selezionarli è utile tenere in conto e mappare l'intero flusso di lavoro. Essere chiari riguardo al flusso di lavoro può anche aiutare a identificare i processi ridondanti così come i maggiori ostacoli. Una delle difficoltà ricorrenti è relativa al fatto che gli strumenti risolvono un problema in un elemento di un flusso di lavoro, solo per creare un problema altrove. Inoltre, le organizzazioni potrebbero avere più flussi di lavoro che potrebbero, a loro volta, avere esigenze diverse che vanno in qualche modo in conflitto. Perciò, la descrizione dei flussi di lavoro rappresenta una base per essere in grado di anticipare le difficoltà e può fornire una tabella di marcia per i continui sviluppi.

\section{Specificare i requisiti}

Al fine di dare una valutazione dell'utilità e del valore dei diversi strumenti disponibili alla propria organizzazione, è utile avere un elenco chiaro dei requisiti. Gli strumenti possono essere comparati e confrontati in modo trasparente e le decisioni giustificate conseguentemente. Quando correttamente eseguite, le attività che mettono insieme i requisiti possono coinvolgere una serie di stakeholder e, di conseguenza, massimizzare il potenziale di allineamento ed efficienza, raggiungendo obiettivi strategici e organizzativi più ampi.

\section{Requisiti che cambiano ed evolvono}

È normale che i requisiti cambino nel corso del tempo. Infatti, la conservazione digitale si occupa in larga misura di far fronte alle difficoltà associate ai cambiamenti inevitabili nelle tecnologie. Pertanto, diviene necessario monitorare e revisionare gli strumenti per far in modo che essi si 
mantengano idonei agli obiettivi e che ogni cambiamento nei requisiti sia reso esplicito. Si raccomanda una revisione periodica della determinazione dei requisiti.

\section{Sostenibilità degli strumenti e partecipazione della comunità}

In ogni decisione da prendere sugli strumenti da utilizzare per la conservazione digitale è importante tenere in conto la sostenibilità. In termini di strumenti, la sostenibilità potrebbe includere una base di utenti attivi, il supporto e lo sviluppo. Ad esempio, un'ampia base di utenti, sia dal punto di vista di fornitori commerciali che open source, può essere un indicatore essenziale per identificare uno strumento sostenibile. È opportuno osservare che una comunità può subire rapidi cambiamenti e per ragioni non facilmente ravvisabili. I "nuovi arrivati" possono diventare rapidamente una tendenza dominante, mentre le grandi comunità possono scemare rapidamente così come le nuove tecnologie che rimpiazzano quelle esistenti. Di conseguenza, potrebbe essere necessario monitorare lo stato di solidità della comunità di sviluppatori che supporta i vostri strumenti.

Trovare gli strumenti di conservazione digitale: strumenti e cataloghi degli strumenti Uno degli aspetti positivi della conservazione digitale negli ultimi due decenni è stato il rapido sviluppo di software, strumenti e servizi che migliorano e consentono i flussi di lavoro della conservazione digitale. Vista la crescita in termini di estensione e varietà della comunità della conservazione digitale, i nostri strumenti sono diventati più potenti e più raffinati. La maggior proliferazione e specializzazione possono anche rappresentare degli ostacoli all'utilizzo: in particolare quando gli strumenti sono il risultato di progetti di ricerca di durata relativamente breve e di portata limitata. Conseguentemente, la diversità degli strumenti può sembrare sempre più disorientante per i nuovi utenti, così come l'accesso al mercato per gli sviluppatori sempre più complesso.

Nel corso degli ultimi anni i cataloghi degli strumenti hanno rappresentato un modo per aiutare gli utenti a individuare quelli di cui hanno bisogno. Ad oggi esistono numerosi registry che descrivono gli strumenti per la conservazione digitale. A seconda degli interessi delle persone che ne sono a capo, questi possono anche fornire descrizioni dettagliate, recensioni o commenti sugli strumenti provenienti dalla comunità più ampia. Quindi, non sono solo utili per gli utenti: consentendo agli esperti di revisionare gli strumenti e valutare la loro performance, indicano i punti di forza e di debolezza e forniscono le basi per sviluppi futuri; collegando gli strumenti agli utenti, aiutano gli sviluppatori a raggiungere un pubblico molto più ampio e ricevere feedback per poter migliorare i loro strumenti.

I registry sono una modalità utilizzata di frequente dalla comunità della conservazione digitale per condividere le informazioni. Ne esistono di diversi tipi come "format registry" che descrivono la performance di determinati formati dei file, o "environment registry" che descrivono lo stack tecnologico necessario per la creazione di un ambiente di esecuzione per emulare o virtualizzare il software. Questi ultimi sono trattati nelle altre sezioni del Manuale. 


\section{Troppi registry?}

Mentre da una parte i registry rappresentano una buona modalità di gestione della proliferazione degli strumenti, è ormai riconosciuto che una proliferazione di registry può essere anche un potenziale ostacolo all'utilizzo. II COPTR registry è stato progettato specificamente per occuparsi di questo problema, basandosi su dati provenienti da diverse fonti tra cui la DCC, POWRR e la Library of Congress.

\section{Supporto pratico e assistenza}

Una volta valutati i registry degli strumenti insieme agli strumenti per la conservazione digitale disponibili per le organizzazioni, l'altra questione che spesso emerge è quale di questi possa conformarsi agli obiettivi della propria organizzazione. Innanzitutto, è importante che la scelta effettuata sia allineata alle esigenze organizzative e alla direzione strategica; le risorse e i casi di studio qui di seguito offrono degli strumenti di valutazione e dei suggerimenti per supportare una corretta implementazione.

\section{Risorse}

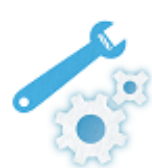

\section{Tools Registry}

\section{Community Owned digital Preservation Tools Registry COPTR}

\section{http://coptr.digipres.org/Main Page}

COPTR descrive gli strumenti utili per la conservazione digitale a lungo termine e agisce principalmente come strumento di ricerca e di valutazione per aiutare i professionisti a trovare gli strumenti di cui hanno bisogno per conservare i dati digitali. L'obiettivo di COPTR è quello di riunire in un unico posto le conoscenze della comunità della conservazione digitale relativa agli strumenti di conservazione. Inizialmente il registry è stato popolato con i dati dei registry gestiti dalle organizzazioni partner di COPTR, inclusi quelli gestiti dal Digital Curation Center, dal Digital Curation Exchange, dalla National Digital Stewardship Alliance, dalla Open Preservation Foundation, dal Preserving digital Objects With Restricted Resources project (POWRR) http: //digitalpowrr.niu.edu/ elencati qui di seguito. COPTR acquisisce gli elementi concreti relativi ad uno strumento, vale a dire, cosa fa, come trovare maggiori informazioni (URL pertinenti) e riferimenti alle esperienze degli utenti nell'utilizzo dello strumento. Lo scopo è un'interpretazione ampia del termine "conservazione digitale". In altre parole, se uno strumento è utile per eseguire una delle funzioni di conservazione digitale come quelle descritte nel modello OAIS o nel DCC lifecycle model, rientra nell'ambito del suddetto registry.

\section{AV Preserve tools list}

\section{http://www.avpreserve.com/avpsresources/tools/}

Un elenco di strumenti di particolare utilità nella conservazione a lungo termine di materiali audiovisivi, sia digitalizzati che nativi digitali. 
Digital Curation Centre (DCC) tools and services list http://www.dcc.ac.uk/resources/external/tools-services

II DCC è un centro di eccellenza, a supporto dei ricercatori nel Regno Unito che affrontano le sfide relative alla conservazione e alla curation delle risorse digitali. Per raggiungere questo obiettivo fornisce una serie di servizi di supporto e consulenza in modo mirato alla ricerca e allo sviluppo. Include un catalogo di strumenti e di servizi che classifica gli strumenti per ricercatori e curatori. Le informazioni sono integrate anche in COPTR (vedi sopra).

\section{DCH-RP registry}

http://www.dch-rp.eu/index.php?en/137/registry-of-services-strumentis

II catalogo degli strumenti della DCH-RP (Digital Cultural Heritage Roadmap for Preservation) raccoglie e descrive le informazioni e le conoscenze relative a strumenti, tecnologie e sistemi che possono essere applicati ai fini della conservazione del patrimonio culturale digitale. La versione 3 del registry è stata creata nel 2014 .

Inventory of FLOSS (Free/libre open-source software) in the cultural heritage domain https://docs.google.com/spreadsheet/ccc?key=0Ag 7rVJwt0CpdFRJOEJxdEk4ZEMxQ01jaDg xQXFSTkE\#gid $=0$

Prodotto dal Progetto Europeana, finanziato dall'UE, questo inventario elenca i software open source gratuiti che potrebbero essere utili nel settore dei beni culturali. Pur non limitandosi agli strumenti per la conservazione digitale, l'inventario contiene informazioni su diversi strumenti con applicazioni nell'ambito della conservazione digitale, valutandone l'obiettivo, la qualità della documentazione, il livello di supporto, i requisiti di licenza e fornendo dei link a informazioni sul progetto e al codice sorgente. Informazioni generali su FLOSS sono disponibili sul sito Europeana http://www.europeana.eu/portal/.

\section{Library of Congress NDIIPP tools showcase \\ http://www.digitalpreservation.gov/tools/}

II catalogo degli strumenti per la conservazione digitale della Library of Congress è un elenco selettivo di strumenti e servizi di interesse per coloro che lavorano nel campo della conservazione digitale. Non viene più gestito attivamente e il contenuto è integrato in COPTR (si veda sopra).

Preserving digital Objects with Restricted Resources (POWRR) Tools Grid http://digitalpowrr.niu.edu/tools-grid/

POWRR ha studiato, valutato e proposto soluzioni di conservazione digitale scalabili e sostenibili per le organizzazioni con una quantità di dati ridotta e/o con meno risorse. Un risultato significativo del progetto è stata la griglia degli strumenti prodotta all'inizio del 2013 sulla base delle categorie funzionali del modello di riferimento OAIS. Attualmente, una versione aggiornata della griglia degli strumenti POWRR può essere generata in COPTR (si veda sopra). 


\section{Digital Preservation Q\&A}

\section{http://qanda.digipres.org/}

Questo è un sito in cui è possibile pubblicare query e risposte per impiegare al meglio gli strumenti, le tecniche, i processi, i flussi di lavoro, le buone pratiche e gli approcci per assicurare l'accesso a lungo termine alle informazioni digitali. La Digital Preservation Q\&A viene attualmente moderata da rappresentanti delle organizzazioni affiliate NDSA e OPF.

\section{Practical e-records}

http://e-records.chrisprom.com/author/prom/

È un blog di Chris Prom sui software e sugli strumenti per archivisti. Sebbene alcune informazioni possano essere datate, il blog fornisce un utile punto di partenza per comprendere gli usi di diversi strumenti per la conservazione digitale e una valutazione standardizzata degli strumenti tenendo conto di una serie di criteri prestabiliti, tra cui facilità di installazione, usabilità, scalabilità, ecc. Oltre alle informazioni sugli strumenti, il blog contiene una miriade di altre risorse utili, inclusi modelli di policy e flussi di lavoro, e approcci consigliati.

\section{Casi di studio}

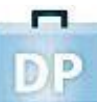

\section{Diary of a repository preservation project \\ http://blog.soton.ac.uk/keepit/}

Presenta un bilancio dei progressi compiuti (tra aprile 2009 e settembre 2010) in quanto il progetto Keeplt, finanziato da JISC, ha affrontato la questione della conservazione dei contenuti dei repository digitali nell'ambito della ricerca, l'insegnamento, la scienza e le arti. Include esperienze utili per la valutazione di strumenti per la conservazione.

\section{Northumberland Estates}

http://wiki.dpconline.org/index.php?title=Northumberland estates case study

Northumberland Estates ha sviluppato un quadro di valutazione semplice per valutare gli strumenti in uso nei vari contesti. II progetto si proponeva di esaminare le possibili opzioni di repository digitali disponibili per le organizzazioni medio-piccole con risorse limitate. È da tenere in considerazione che le indicazioni attese nel caso aziendale finale riflettono le esigenze organizzative di Northumberland Estates e potrebbero non soddisfare le esigenze della propria organizzazione. Il caso di studio è stato preparato nell'ambito del progetto SPRUCE finanziato da JISC. 


\section{Fixity e checksum}

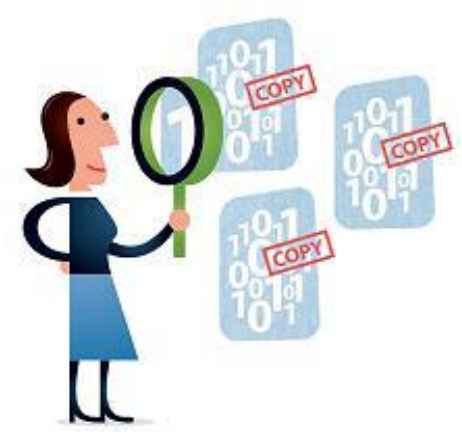

Illustrazione di Jørgen Stamp digitalbevaring.dk CC BY 2.5 Denmark

\section{Fixity}

"Fixity, in the preservation sense, means the assurance that a digital file has remained unchanged, ovvero fixed." (Bailey, 2014). La Fixity non si applica solo ai file, ma a qualsiasi oggetto digitale che contiene una serie di bit in cui quel "bitstream" deve essere mantenuto intatto con la consapevolezza che non è cambiato. La Fixity potrebbe essere applicata alle immagini o ai video all'interno di un oggetto audiovisivo, ai singoli file all'interno di uno zip, ai metadati all'interno di una struttura XML, ai record in un database o agli oggetti in un object store. Tuttavia, il modo più comune per archiviare materiali digitali è attraverso i file e la Fixity dei file può essere stabilita e monitorata mediante l'uso dei checksum.

\section{Checksum}

Un checksum di un file è un'“impronta digitale" in base alla quale anche la più piccola modifica al file farà sì che il checksum cambi completamente. Generalmente, i checksum sono creati utilizzando tecniche crittografiche e possono essere generati utilizzando una gamma di strumenti prontamente disponibili e open source. È importante notare che mentre i checksum possono essere usati per rilevare se il contenuto di un file è cambiato, non indicano la posizione nel file in cui è avvenuta la modifica.

I checksum hanno tre usi principali:

1. Sapere che un file è stato ricevuto correttamente dal proprietario del contenuto o dalla fonte e successivamente trasferito correttamente all'archivio di conservazione.

2. Sapere che la fixity dei file è stata garantita durante l'archiviazione.

3. Da fornire successivamente agli utenti del file in modo che sappiano che il file è stato correttamente recuperato dall'archivio e consegnato loro.

Ciò consente di stabilire una "catena di custodia" tra coloro che producono o forniscono materiali digitali, i responsabili dell'archiviazione continua e coloro che devono utilizzare il materiale digitale che è stato archiviato. Nel modello di riferimento OAIS (ISO, 2012) questi sono: i produttori, l'OAIS stesso che è il repository, e gli utenti. 


\section{Applicazione nella conservazione digitale}

Se un'organizzazione ha più copie dei propri file, ad esempio come raccomandato nella sezione Archiviazione, è possibile utilizzare i checksum per monitorare la Fixity di ogni copia di un file e, se una delle copie è cambiata, un'altra può essere usata per creare un buon sostituto. L'approccio consiste nel calcolare un nuovo checksum per ogni copia di un file su base regolare e confrontarlo con il valore di riferimento noto per essere corretto. Se viene rilevata una deviazione, si capisce che il file è stato corrotto in qualche modo e dovrà essere sostituito con una nuova copia valida. Questo processo è noto come "scrubbing dei dati".

I checksum sono ideali per rilevare se sono state apportate modifiche indesiderate ai materiali digitali. Tuttavia, a volte i materiali digitali possono essere modificati deliberatamente, ad esempio se viene migrato un formato di file. Ciò provoca la modifica del checksum e richiede la creazione di nuovi checksum dopo la migrazione, che diventano il modo per verificare l'integrità dei dati del nuovo file in futuro.

I checksum dei file dovrebbero essere controllati regolarmente. La frequenza con cui eseguire i controlli dipende da molti fattori, tra cui il tipo di archiviazione, la sua manutenzione e la frequenza di utilizzo. Come linea guida generale, il controllo dei nastri dati potrebbe essere eseguito ogni anno e il controllo dei sistemi basati su disco rigido potrebbe essere effettuato ogni sei mesi. Controlli più frequenti consentono di rilevare e risolvere i problemi prima, ma a scapito di un maggiore carico sul sistema di archiviazione e di maggiori risorse di elaborazione.

I checksum possono essere archiviati in vari modi, ad esempio all'interno di un record PREMIS, in un database $\mathrm{o}$ in un "manifest" che accompagna i file in un sistema di archiviazione.

II supporto degli strumenti è utile per la generazione e l'utilizzo del checksum. Trattandosi di funzioni relativamente semplici, i checksum sono integrati in molti altri strumenti di conservazione digitale. Ad esempio, la generazione di checksum come parte del processo di ingest e l'aggiunta di queste informazioni di fixity ai pacchetti di archiviazione generati, oppure la possibilità di generare manifest di checksum per più file e il raggruppamento di manifest e file per un facile trasporto o archiviazione. Inoltre, md5sum e md5deep forniscono semplici strumenti da riga di comando che operano su più piattaforme per generare checksum su singoli file o directory.

Esistono diversi algoritmi di checksum, ad es., MD5 e SHA-256, che possono essere utilizzati per generare checksum più forti. Quanto più "forte" è l'algoritmo, tanto più difficile è modificare deliberatamente un file in modo che non venga rilevato. Ciò può essere importante per le applicazioni in cui è necessario dimostrare resistenza alla corruzione malevola $\mathrm{o}$ all'alterazione delle risorse digitali, ad esempio nei casi in cui il peso probatorio e l'ammissibilità legale sono importanti. Tuttavia, se vengono utilizzati checksum per rilevare perdite accidentali o danni ai file, ad esempio a causa di un errore di archiviazione, MD5 è sufficiente e ha il vantaggio di essere ben supportato dagli strumenti, ed è veloce da calcolare. 
II Manuale segue i livelli di conservazione della National Digital Stewardship Alliance (NDSA) (NDSA, 2013) nel raccomandare quattro livelli in corrispondenza dei quali la conservazione digitale può essere supportata attraverso tecniche di fixity dei file e integrità dei dati. Molti dei vantaggi del controllo della fixity possono essere ottenuti solo se esistono più copie dei materiali digitali, ad esempio consentendo la riparazione in caso di perdita dell'integrità di una delle copie.

\begin{tabular}{|c|c|c|}
\hline Livello & Attività & Rischi affrontati e benefici raggiunti \\
\hline 1 & $\begin{array}{l}\text { - Controllare la fixity dei file al momento } \\
\text { dell'ingest se è stato fornito insieme al } \\
\text { contenuto. } \\
\text { - Creare le informazioni sulla fixity se } \\
\text { non sono state fornite insieme al } \\
\text { contenuto. }\end{array}$ & $\begin{array}{l}\text { - I materiali digitali danneggiati o errati non } \\
\text { vengono archiviati consapevolmente. } \\
\text { - L'autenticità dei materiali digitali può essere } \\
\text { affermata. } \\
\text { - Si stabilisce la fixity di base, pertanto è } \\
\text { possibile che vengano rilevate modifiche } \\
\text { indesiderate dei dati. }\end{array}$ \\
\hline 2 & $\begin{array}{l}\text { - Controllare la fixity su tutti gli ingest } \\
\text { - Usare i programmi di blocco di scrittura } \\
\text { quando si lavora con il supporto } \\
\text { originale } \\
\text { Eseguire il controllo antivirus di } \\
\text { contenuti ad alto rischio. }\end{array}$ & $\begin{array}{l}\text { - Nessun materiale digitale di integrità non } \\
\text { confermata può entrare nell'archivio di } \\
\text { conservazione. II peso probatorio viene } \\
\text { supportato per autenticità. } \\
\text { - È possibile garantire a tutti i produttori che } \\
\text { il loro contenuto è stato ricevuto in modo } \\
\text { sicuro. Il supporto originale è protetto. } \\
\text { - Nessun contenuto dannoso può accedere } \\
\text { all'archivio di conservazione }\end{array}$ \\
\hline 3 & $\begin{array}{l}\text { - Verificare periodicamente la fixity dei } \\
\text { contenuti presenti nei sistemi di } \\
\text { archiviazione e conservazione. } \\
\text { - Mantenere i log delle informazioni sulla } \\
\text { fixity e fornire audit su richiesta. } \\
\text { - Capacità di rilevare dati danneggiati. } \\
\text { - Eseguire il controllo antivirus di tutto il } \\
\text { contenuto. }\end{array}$ & $\begin{array}{l}\text { - Protezione da un'ampia gamma di eventi di } \\
\text { corruzione e perdita di dati. I problemi con } \\
\text { l'archiviazione sono stati rilevati in } \\
\text { precedenza. } \\
\text { - La corruzione o la perdita dei dati non viene } \\
\text { rilevata a causa di "errori silenziosi" o "guasti } \\
\text { non rilevati". I materiali digitali non sono in } \\
\text { uno stato di integrità "sconosciuta". } \\
\text { - È possibile attribuire un peso probatorio } \\
\text { costante al fatto che i materiali digitali siano } \\
\text { intatti e corretti. }\end{array}$ \\
\hline 4 & $\begin{array}{l}\text { - Verificare la fixity di tutto il contenuto in } \\
\text { risposta a eventi o attività specifici } \\
\text { - Capacità di sostituire / riparare i dati } \\
\text { danneggiati. }\end{array}$ & $\begin{array}{l}\text { - Le modalità di guasto che minacciano i } \\
\text { materiali digitali vengono contrastate in } \\
\text { modo proattivo. Tutte le copie dei materiali } \\
\text { digitali vengono mantenute attivamente. }\end{array}$ \\
\hline
\end{tabular}




\begin{tabular}{|l|l|l|}
\hline $\begin{array}{l}\text { - Accertarsi che nessuno abbia accesso } \\
\text { in scrittura a tutte le copie. }\end{array}$ & $\begin{array}{l}\text { - Assicurazione per gli utenti dell'integrità e } \\
\text { dell'autenticità dei materiali digitali a cui si } \\
\text { accede. }\end{array}$ \\
& $\begin{array}{l}\text { - L'efficacia dell'approccio alla conservazione } \\
\text { può essere misurata e dimostrata. }\end{array}$ \\
& $\begin{array}{l}\text { Conformità agli standard, ad esempio, ISO } \\
16363 \text { Audit e certificazione di repository } \\
\text { digitali affidabili. }\end{array}$ \\
\hline
\end{tabular}

\section{Blocco dei diritti di scrittura}

È da tenere presente che la National Digital Stewardship Alliance (NDSA) raccomanda l'uso di strumenti di blocco dei diritti di scrittura al livello 2. Questo per impedire l'accesso in scrittura ai supporti su cui i materiali digitali potrebbero trovarsi prima di essere stati copiati nel sistema di conservazione. Ad esempio, se il materiale digitale viene consegnato a un'organizzazione su un disco rigido o una chiave USB, un blocco dei diritti di scrittura impedirebbe l'eliminazione accidentale del materiale digitale quando l'unità o la chiave vengono letti. II materiale digitale potrebbe non essere su supporto fisico, ad esempio potrebbe essere su un server di archiviazione obsoleto o consegnato tramite un trasferimento di rete, ad esempio un caricamento ftp. In questi casi non si applicherebbe il blocco dei diritti di scrittura e verrebbero utilizzate altre misure per rendere il materiale digitale "di sola lettura" e quindi immutabile prima della conferma che il materiale digitale è stato trasferito con successo all'archivio di conservazione. Inoltre, il blocco dei diritti di scrittura non esiste per tutti i tipi di supporto. Se effettivamente un dispositivo di blocco dei diritti di scrittura è applicabile, le competenze o i costi necessari per utilizzarlo dovrebbero essere valutati in rapporto al rischio dei danni al materiale digitale originale o alla necessità di disporre di una autenticità dei dati rigorosa. Pertanto, alcune organizzazioni potrebbero considerare non necessario l'uso del blocco dei diritti di scrittura oppure un passaggio al livello 3 o livello 4.

\section{Risorse

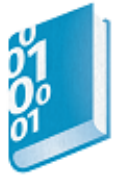

Bailey, J., 2014, Protect Your Data: File Fixity and Data Integrity, The Signal, Library of Congress.

http://blogs.loc.gov/digitalpreservation/2014/04/protect-your-data-file-fixity-and-data-integrity/

\section{Checking Your Digital Content: What is Fixity and When Should I Be Checking It?} http://digitalpreservation.gov/ndsa/working groups/documents/NDSA-Fixity-Guidance-Reportfinal100214. pdf? loclr=blogsig

Molti nella comunità della conservazione conoscono la necessità di dover verificare la fixity dei loro contenuti, ma come, quando e con quale frequenza? Questo documento pubblicato dalla 
NDSA nel 2014 ha lo scopo di aiutare gli amministratori a fornire le risposte a queste domande in base alle esigenze e alle risorse della loro organizzazione (7 pagine).

\section{AVPreserve Fixity Tools \\ http://www.avpreserve.com/tools/fixity/}

\section{MD5}

https://tools.ietf.org/html/rfc1321

\section{SHA-1}

http://csrc.nist.gov/publications/fips/fips180-4/fips-180-4.pdf

\section{SHA-256}

http://csrc.nist.gov/groups/STM/cavp/documents/shs/sha256-384-512.pdf

\section{Md5deep and hashdeep}

http://coptr.digipres.org/Md5deep and hashdeep

\section{md5sum}

http://coptr.digipres.org/Md5sum Unix command

\section{The "Checksum" and the Digital Preservation of Oral History}

https://www.youtube.com/watch?v=Emom ncMqu0

Una buona panoramica che non si limita alla storia orale, questo video fornisce una breve introduzione al ruolo del checksum nella conservazione digitale. Presenta Doug Boyd, direttore del Louie B. Nunn Centre for Oral History presso la University of Kentucky Libraries. (3 minuti 25 secondi)

\section{Riferimenti}

Bailey, J., 2014. Protect Your Data: File Fixity and Data Integrity. The Signal. [blog]. Disponibile al seguente link: http://blogs.loc.gov/digitalpreservation/2014/04/protect-your-data-file-fixityand-data-integrity/

ISO, 2012. ISO 14721:2012 - Space Data and Information Transfer Systems - Open Archival Information System (OAIS) - Reference Model, 2nd edn. Geneva: International Organization for Standardization. Disponibile al seguente link: 
http://www.iso.org/iso/iso catalogue/catalogue tc/catalogue detail.htm?csnumber=57284

NDSA, 2013. The NDSA Levels of Digital Preservation: An Explanation and Uses, version 1 2013. National Digital Stewardship Alliance. Disponibile al seguente link: http://www.digitalpreservation.gov/ndsa/working groups/documents/NDSA Levels Archiving 2013.pdf 


\section{Formati dei file e standard}

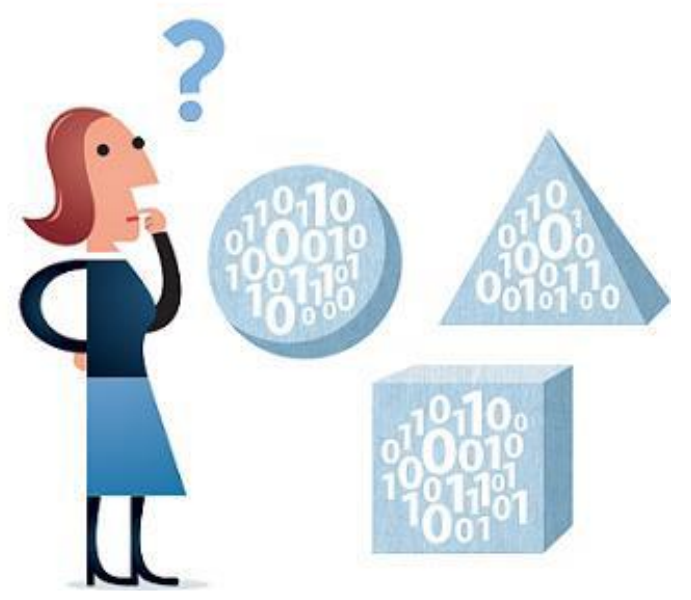

Illustrazione di Jørgen Stamp digitalbevaring.dk CC BY 2.5 Denmark

\section{Introduzione}

La gestione dei formati dei file dovrebbe essere valutata nel più ampio contesto strategico della pianificazione della conservazione. Cosa può permettersi la propria organizzazione? Quanto impegno richiederà da parte dello sviluppatore? Cosa richiedono gli utenti dalle collezioni? Si sta per affrontare un problema di archiviazione? Ad ogni modo, la risposta alle questioni sulla conservazione digitale è quella di cercare di non "fare tutto". La strategia prescelta dovrebbe basarsi su azioni semplici e pratiche, piuttosto che tentare di supportare più formati file rispetto a quelli di cui si ha necessità.

L'obiettivo di questa sezione non è quello di fornire un elenco dettagliato o esaustivo dei formati attuali per i diversi tipi di contenuto, ma di focalizzarsi sulle implicazioni più generali dei formati dei file per la loro applicazione e sulle implicazioni per la conservazione.

Parte sostanziale di questa sezione fa riferimento alla possibile selezione di un formato di file nella prospettiva di migrazione. Sebbene la migrazione sia una valida strategia di conservazione, e anche piuttosto comune per molti formati file, non è l'unico approccio o soluzione. Ove opportuno, in questa sezione si farà riferimento ad altri metodi adatti per la conservazione.

\section{Formati dei file organizzati per tipi di contenuto}

Diversi tipi di contenuto hanno sviluppato nel tempo propri formati dei file nel tentativo di adattare le specifiche funzionalità alle loro esigenze. Le principali tipologie di contenuto sono rappresentate da immagini, video, audio e testo; tuttavia, un numero crescente di formati viene sviluppato per soddisfare le esigenze dei nuovi media, inclusi i formati per i modelli 3D e l'archiviazione del Web. 
I formati dei file variano enormemente in termini di complessità, con alcuni dati codificati in molti livelli. In alcuni casi i formati dei file rientrano in un contesto più ampio, un contesto che include software, hardware e persino interi ambienti informativi.

Per ulteriori raccomandazioni sulla conservazione di specifici tipi di contenuto digitale e i formati dei file associati, si consiglia di consultare i casi di studio nel Manuale sulla Conservazione di contenuti specifici.

\section{Formati dei file: di cosa dovremmo preoccuparci? Obsolescenza}

I formati si evolvono man mano che gli utenti e gli sviluppatori identificano e incorporano nuove funzionalità. Nuovi formati o versioni di formati possono portare all'obsolescenza dei formati dei file poiché le nuove generazioni di software eliminano gradualmente il supporto per i formati più vecchi. Quando il software non prevede la retrocompatibilità con formati dei file meno recenti, i dati potrebbero diventare inutilizzabili. Sia i formati open source che quelli commerciali sono particolarmente esposti all'obsolescenza: i fornitori a volte usano l'obsolescenza pianificata per invogliare i clienti a passare a nuovi prodotti, mentre le comunità di software open source possono ritirare il supporto per i formati più vecchi se questi non vengono più richiesti dalla comunità. Anche l'obsolescenza può essere accidentale: sia le aziende che le comunità open source possono fallire.

L'obsolescenza del formato dei file è un rischio da non sottovalutare. Ciò detto, il problema potrebbe non essere così grave come era stato percepito circa 10 anni fa dalla comunità della conservazione digitale. Molti formati dei file consolidati esistono ancora, sono ancora supportati

ed utilizzabili. È molto probabile che la maggior parte dei formati dei file di cui ci si occupa siano ben supportati.

\section{Proliferazione}

Probabilmente, in alcuni settori, la proliferazione può essere considerata una sfida ancor più grande rispetto all'obsolescenza. Se i formati non sono normalizzati, un'organizzazione può ritrovarsi con un gran numero di formati diversi e versioni di file: ad esempio, diverse versioni di PDF, word, formati immagine, ecc. Questo problema è più evidente nei domini che sono in rapida evoluzione come quelli che sviluppano formati di dati personalizzati. Tracciare e gestire tutti questi formati - quali sono quelli a rischio, e quali strumenti possono essere utilizzati per ciascuno di essi - può rappresentare una sfida importante.

La strategia di conservazione digitale perseguita dovrebbe avere come obiettivo quello di mitigare gli effetti dell'obsolescenza e della proliferazione. Tenendo conto del contesto delle proprie collezioni e della propria organizzazione, strategie come la migrazione, l'emulazione, la normalizzazione e un'attenta selezione dei formati dei file sono tutte valide e da prendere in considerazione. 


\section{Gli aspetti dei formati dei file per la conservazione digitale}

\section{Selezione dei formati di destinazione per la conservazione}

Non tutti i formati digitali sono adatti o progettati per l'archiviazione o la conservazione. Qualsiasi strategia di conservazione dovrebbe quindi tenere in conto i requisiti del contenuto della collezione e decidere su un formato di file che meglio preservi tali qualità. Associare i contenuti ad una scelta adeguata di formato di conservazione o formato di accesso; identificare ciò che è importante nel contenuto.

Qui di seguito si suggeriscono alcuni fattori da tenere in considerazione nella selezione dei formati dei file preferiti:

\section{Open source contro proprietario?}

I formati open source, come JPEG2000, sono molto popolari per la loro natura non proprietaria e per il senso di titolarità che gli stakeholder possono conseguire con il loro utilizzo. Tuttavia, la scelta dei formati open source rispetto a quelli proprietari non è così semplice e deve essere esaminata attentamente. I formati proprietari, come TIFF, sono considerati molto robusti; tuttavia, questi formati saranno alla fine oggetto di aggiornamenti e obsolescenza se il proprietario fallisce o sviluppa una nuova alternativa. Allo stesso modo, i formati open source possono essere visti come tecnologicamente neutri, non dipendenti dai modelli di business per il loro sviluppo, ma possono anche essere visti come vulnerabili alle suscettibilità delle comunità che li supportano.

Sebbene i formati non proprietari possano essere selezionati per molti tipi di risorse, ciò non si verifica su scala generale. Per molte nuove aree e applicazioni, ad esempio Geographical Information Systems o Virtual Reality, sono disponibili solo formati proprietari. In tali casi un fattore cruciale sarà il formato di esportazione supportato per consentire ai dati di essere spostati fuori da (o dentro a) questi ambienti proprietari.

\section{Documentazione e standard}

La disponibilità della documentazione, ad esempio le specifiche pubblicate, è un fattore importante nella scelta di un formato di file. La documentazione può esistere sotto forma di specifiche del fornitore, di uno standard internazionale o può essere creata e gestita nel contesto di una comunità di utenti. È importante cercare uno standard ben documentato e ampiamente implementato. Bisogna assicurarsi che lo standard sia elencato nel registry di formati dei file PRONOM.

\section{Adozione}

Se un ampio gruppo di utenti fa affidamento su un formato di file, questo genera molte più soluzioni per i suoi utenti. Vale la pena tenere presente i livelli di uso e il supporto per i formati nel resto del mondo, ma anche scoprire cosa stanno facendo organizzazioni simili a quella di pertinenza e condividere le buone pratiche nella selezione dei formati. L'ampia adozione di un formato può dare più sicurezza nella propria strategia di conservazione. 


\section{Lossless contro lossy}

I formati lossy sono quelli in cui i dati vengono compressi o modificati durante il processo di codifica. II formato MP3 è ampiamente utilizzato per la distribuzione commerciale di file musicali sul Web, poiché i metodi di codifica lossy hanno come risultato un file di dimensioni inferiori.

TIFF è un esempio di un formato immagine in grado di supportare dati lossless. Potrebbe contenere un'immagine ad alta risoluzione. JPEG è un esempio di un formato di file di immagine lossy, la sua versatilità e le dimensioni ridotte del file lo rendono una scelta adatta per la creazione di una copia di accesso di un'immagine di dimensioni inferiori per la trasmissione in rete. Non sarebbe appropriato archiviare l'immagine JPEG come formato di accesso e di archiviazione visto che ciò comporterebbe la perdita irrecuperabile di dati.

Una regola generale potrebbe essere quella di scegliere formati lossless per la creazione e l'archiviazione di "archival masters"; i formati lossy devono essere utilizzati solo a fini di consegna/accesso e non devono essere considerati come archivi. Questa regola è particolarmente adatta per un progetto di digitalizzazione, in particolare di immagini fisse.

\section{Supporto per i metadati}

Alcuni formati dei file supportano i metadati. Ciò significa che alcuni metadati possono essere inseriti direttamente in un'istanza di un file (ad esempio, JPEG2000 supporta alcuni campi di metadati relativi ai diritti). Questo è un aspetto che si può tenere in considerazione a seconda dell'approccio che si è scelto per la gestione dei metadati.

\section{Proprietà significative dei formati dei file}

Questo è un aspetto complesso: da un lato, le proprietà significative possono essere considerate "l'essenza" del contenuto del file; una strategia che arriva al cuore di "cosa preservare". Cosa si aspetta la comunità di utenti dalla riproduzione? Quali aspetti dell'originale si sta cercando di preservare? Questa strategia potrebbe comportare il fatto che non ci si debba impegnare a preservare tutti gli aspetti di un formato di file, ma solo quelli che hanno più significato e valore per l'utente.

Le proprietà significative possono anche riferirsi a una gamma molto specifica di metadati tecnici che devono essere presenti per poter riprodurre un file (ad esempio, la larghezza dell'immagine). Alcuni strumenti di migrazione possono eliminare questi metadati, o questi ultimi potrebbero andare persi attraverso azioni di curation nel repository. La strategia di conservazione deve impedire che si verifichi questa perdita. Diventa quindi importante identificare, estrarre, archiviare e preservare le proprietà significative nelle prime fasi del processo di conservazione.

\section{Cose che possiamo fare}

Diverse sono le cose che si potrebbero fare per supportare i formati dei file nel proprio archivio digitale e ci sono molti strumenti disponibili per sostenere queste attività. Attualmente ne esistono così tanti che sono stati sviluppati dei cataloghi degli strumenti di conservazione digitale per aiutare a localizzarli e valutarli (si riporta alle sezioni Strumenti e Risorse). 


\section{Strumenti per la migrazione}

In generale, si tratta di strumenti che trasformano un formato di file da un formato obsoleto in un formato più recente che può essere supportato. Esistono molti strumenti per eseguire questa migrazione, e tendenzialmente si limitano a svolgere una sola operazione (ad esempio ImageMagick funziona solo per le immagini digitali).

Uno strumento di migrazione è solo una parte di un percorso di migrazione. II percorso deve includere un formato destinazione, che sarà stato selezionato conformemente alle indicazioni precedentemente menzionate.

Gli strumenti di migrazione possono presentare dei rischi. Uno di questi è rappresentato dalle modifiche "invisibili" al contenuto o ai dati durante la migrazione. Per ridurre questo rischio, una strategia potrebbe essere quella di ideare una serie di criteri di accettazione relativi a ciò che l'oggetto trasformato deve conservare, ad esempio, in termini di formattazione, di aspetto grafico o anche di funzionalità e confermare i risultati desiderati con un processo di garanzia della qualità.

La migrazione del formato di/del file non è sempre la soluzione, ad esempio alcuni formati dei file CAD e CAM non possono essere migrati facilmente. L'industria aerospaziale ha scoperto che la migrazione di file CAD meno recenti in un formato più recente richiede un processo di validazione, principalmente perché sono richiesti dal quadro normativo per dimostrare che i loro dati sono validi e soddisfano standard rigorosi. In breve, il costo della migrazione e della validazione è (per loro) molto più elevato di una soluzione di emulazione, un approccio che (in questo caso) comporta la conservazione del software CAD e la sua manutenzione.

Si vedano anche le sezioni sugli Strumenti e Conservazione di contenuti specifici.

\section{Strumenti per il rendering}

In generale, si tratta di strumenti in grado di leggere e riprodurre un formato di file, in modo che la comunità di utenti possa leggere e interpretare la risorsa; è più comunemente applicato ai file archiviati in formati accessibili. Uno strumento di rendering di base è/potrebbe essere PDF Reader, uno più sofisticato il lettore multimediale Wellcome Library, che supporta testi OCR, immagini e file audiovisivi.

\section{Strumenti per l'identificazione del formato di/dei file}

Strumenti in grado di identificare gli aspetti dei formati dei file che non sono immediatamente evidenti a partire dalla loro estensione. Lo fanno leggendo l'intestazione del formato di/dei file e quindi possono identificare ad esempio il tipo MIME, la dimensione e la versione. Esempi di tali strumenti includono PRONOM, JHOVE e NZ Metadata Extraction Tools (si veda Risorse qui di seguito).

Questi strumenti vengono impiegati al momento dell'ingest, in modo da sapere fin dall'inizio che tipo di formati dei file si sta acquisendo nell'archivio. 
Alcuni strumenti di identificazione possono anche indicare strumenti di rendering o persino (come PRONOM) suggerire un percorso di migrazione basato sull'identificazione del formato dei file.

\section{Strumenti per la verifica del formato file}

JHOVE è uno dei pochi strumenti in grado di convalidare un formato di file. Funziona confrontando un'istanza di un formato di file con insiemi di comportamenti previsti, che memorizza nella sua libreria. JHOVE può fornire dei resoconti su determinati formati dei file e stabilirne la validità e la buona realizzazione.

\section{Sondaggi sulle collezioni}

Passare in rassegna i formati dei file in uso / conoscere ciò che si ha / caratterizzare le proprie collezioni: ciò si ricollega nuovamente a una strategia di pianificazione, che consente di sapere cosa si deve supportare e il probabile sforzo richiesto per farlo.

Un'indagine dovrebbe prestare particolare attenzione alle versioni dei formati dei file e al software necessario per il loro rendering. Si suggerisce, se possibile, di raccogliere qualsiasi informazione sulle specifiche pubblicate per questi formati; alcune di queste sono pubblicate sul web.

Tra i lavori utili in questo settore ci sono quelli svolti presso la British Library, con progetti sulla valutazione della sostenibilità (Maureen Pennock, Paul Wheatley, Peter May) e Collection Profiling (Michael Day, Maureen Pennock, Ann MacDonald). Al momento della stesura di questo documento non esistono link attivi a questi progetti, ma si prevede che il lavoro di valutazione della sostenibilità sarà pubblicato sul wiki della DPC. Questi sono approcci utili e possono essere considerati esempi di buone pratiche esistenti. Sebbene non si scenda nello stesso dettaglio della BL nella valutazione o nella profilazione, è un esercizio pratico e valido.

\section{Evitare la proliferazione dei tipi di file}

Ove possibile, ridurre la gamma di formati dei file supportati, al fine di ridurre la complessità. Un approccio valido alla pianificazione della conservazione è la normalizzazione, invece dell'aggiunta di più formati di migrazione alle proprie collezioni. Minore è la serie di formati, minori sono le spese generali.

\section{Comunità}

Identificare un accordo consensuale sui formati dei file di destinazione; collaborare con gli enti che detengono collezioni simili alle proprie. Con quali formati scelgono di lavorare?

\section{Conclusione}

Per alcuni tipi di contenuto, vi è consenso sulla scelta del formato di conservazione. Ad esempio, nell'archiviazione audio per la quale viene comunemente usato WAV. In altre aree il consenso è molto più difficile da raggiungere. La conservazione dei video digitali è un'area complessa in cui i progressi sono stati ostacolati da una mancanza di accordi e da una proliferazione 
incontrollata di formati di wrapper, metodi di consegna e metodi di codifica. La scelta dei formati dei file immagine è leggermente più chiara, con una scelta limitata di formati per l'archiviazione e altri per la consegna. È stato generalmente accettato che il formato TIFF rappresenti il formato corretto per l'archiviazione dei file master (anche i formati RAW o DNG sono considerati appropriati per l'archiviazione), ma attualmente si sta prendendo in considerazione il formato JPEG2000 che fornisce un livello maggiore di compressione lossless rispetto al TIFF, ed è open source.

\section{Risorse

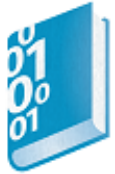

Library of Congress recommended format specifications

http://www.loc.gov/preservation/resources/rfs/index.html

Sviluppa una serie di specifiche di formati che raccomanda, sia internamente ai propri professionisti che esternamente a creatori, venditori e archivisti, di utilizzare per garantire la conservazione e l'accesso a lungo termine. Tratta sia i formati digitali che analogici ed è divisa in sei grandi categorie: Opere testuali e Composizioni musicali; Immagini; Audio; Film; Software e giochi elettronici e e-learning; e set di dati/database.

Jisc significant properties reports

Tra il 2007 e il 2008 JISC ha finanziato cinque studi sulle proprietà significative per diversi tipi di contenuti e file. Si noti che la discussione nelle relazioni va dal 2007 al 2008. Le relazioni sono le seguenti:

inSPECT Significant Properties Report 2007 (10 pagine)

http://citeseerx.ist.psu.edu/viewdoc/download?doi=10.1.1.109.7923\&rep=rep1\&type=pdf

Significant Properties of E-learning Objects 2008 (65 pagine)

http://www.webarchive.org.uk/wayback/archive/20140616090345/http://www.jisc.ac.uk/media/ documents/programmes/preservation/spelos report.pdf

The Significant Properties of Moving images 2008 (62 pagine)

http://www.webarchive.org.uk/wayback/archive/20140616090254/http://www.jisc.ac.uk/media/ documents/programmes/preservation/spmovimages report.pdf 19

The Significant Properties of Software: A Study 2008 (97 pagine)

http://www.webarchive.org.uk/wayback/archive/20100624233431/http://www.jisc.ac.uk/media/ documents/programmes/preservation/spsoftware report redacted.pdf

The Significant Properties of Vector Images 2007 (61 pagine)

http://www.webarchive.org.uk/wayback/archive/20140616090304/http://www.jisc.ac.uk/media/ documents/programmes/preservation/vector images.pdf 


\section{British Library File Formats Assessments}

\section{http://wiki.dpconline.org/index.php?title=File Formats Assessments}

II Digital Preservation Team della British Library ha avviato delle valutazioni sul rischio della conservazione dei formati dei file per rilevare le lacune nelle buone pratiche attuali, acquisire la comprensione e la capacità di lavorare con formati dei file specifici. Il focus di ogni valutazione è capire i rischi della conservazione basati sull'evidenza e sulle implicazioni dell'obsolescenza istituzionale che portano a problemi nel mantenere il contenuto nel tempo. Le valutazioni sono pubblicate in una nuova sezione della Wiki DPC. Tre valutazioni riguardanti JP2, TIFF e PDF hanno dato il via alla serie.

\section{Library of Congress sustainability factors}

\section{http://www.digitalpreservation.gov/formats/index.shtml}

Questo sito riguarda i formati associati al contenuto digitale indipendente dal supporto, vale a dire il contenuto che è generalmente gestito come file e che non dipende da un particolare supporto fisico. Non si occupa dei formati associati ai contenuti digitali dipendenti dal supporto, ovvero formati dipendenti e indissolubilmente collegati a supporti fisici, ad esempio DVD, CD audio e formati di videocassette come DigiBeta. Identifica e descrive i formati che sono utili alla sostenibilità a lungo termine e sviluppa strategie per sostenere questi formati, comprese le raccomandazioni relative agli strumenti e alla documentazione necessari per la loro gestione.

\section{Jisc digital media infokit: digital file formats}

http://www.jiscdigitalmedia.ac.uk/infokit/file formats/digital-file-formats

II Jisc Digital Media Infokit ha come obiettivo quello di fornire risposte rapide e pratiche a "quale formato di file dovrei usare per ...?" Tratta i formati di immagini, audio e video, nonché le attività e le applicazioni comuni in ambito educativo e culturale.

\section{Help Solve the File Format Problem}

http://fileformats.archiveteam.org

Un wiki di informazioni crowd-sourced sui formati dei file che si trova sul sito dell'Archive Team. Tutto il contenuto è disponibile con licenza Creative Commons 0.

Is JPEG 2000 a digital preservation risk?

http://blogs.loc.gov/digitalpreservation/2013/01/is-ipeg-2000-a-preservation-risk/ 20

Un interessante blog ospite e filone di discussione riferito al formato immagine JPEG 2000. 


\section{OPF File Format Risk Registry}

http://wiki.opf-labs.org/display/TR/OPF+File+Format+Risk+Registry

Si concentra in particolare sui problemi e sui rischi relativi al formato dei file che hanno implicazioni per la conservazione e l'accessibilità a lungo termine e su come gestirli in modo pratico. Mira ad essere complementare a format registry più formali.

\section{PRONOM}

http://apps.nationalarchives.gov.uk/pronom/Default.aspx

Questo file format registry è una risorsa importante per chiunque necessiti di informazioni imparziali e definitive sui formati dei file, prodotti software e altri componenti tecnici necessari per supportare l'accesso a lungo termine a documenti elettronici e altri oggetti digitali di valore culturale, storico o commerciale.

\section{DROID (Digital Record Object Identification)}

http://www.nationalarchives.gov.uk/information-management/manage-information/preservingdigital-records/droid/

Questo è uno strumento di identificazione automatica del formato di file che fornisce categorie di identificazione del formato per file sconosciuti in una collezione digitale. Utilizza firme interne per identificare e segnalare il formato e la versione specifici dei file digitali. Tali firme sono archiviate in un file di firma XML, generato dalle informazioni registrate nel registro PRONOM.

\section{Casi di Studio}

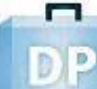

Per i casi studio pertinenti, consultare la sezione Casi di studio dettagliati sulla conservazione dei contenuti del Manuale. 


\section{Sicurezza delle Informazioni}

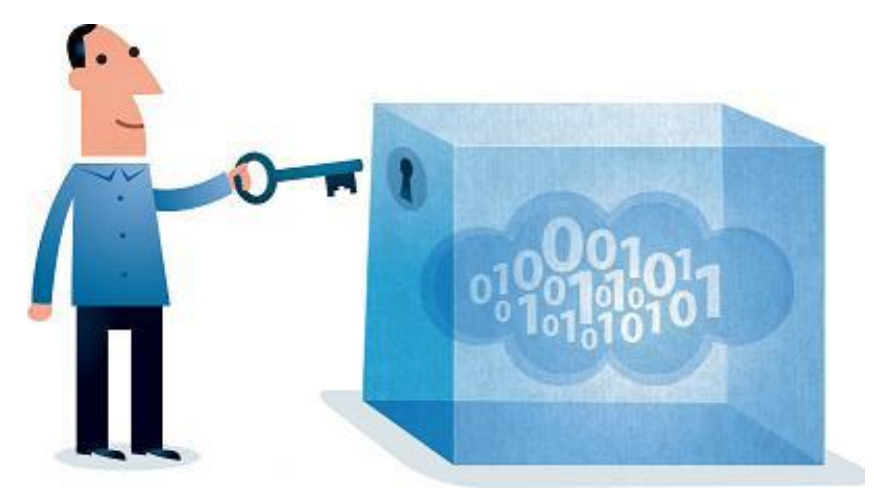

Illustrazione di Jørgen Stamp digitalbevaring.dk CC BY 2.5 Denmark

\section{Introduzione}

Questa sezione rappresenta una guida, per i professionisti a livello principiante o intermedio, sulle implicazioni della sicurezza delle informazioni per la conservazione digitale. Le questioni sulla Sicurezza delle informazioni riguardano la sicurezza del sistema (ad esempio, protezione della conservazione digitale e dei sistemi/servizi in rete dall'esposizione a minacce esterne/interne); la sicurezza della collezione (ad esempio, la protezione del contenuto da perdita o modifica, autorizzazione e verifica dei processi di repository); e gli aspetti legali e normativi (ad esempio, le informazioni personali o riservate nel materiale digitale, accesso sicuro, revisione). La sicurezza delle informazioni è un argomento complesso e importante per i sistemi di informazione in generale. È importante fare affidamento sulle competenze sia all'interno che all'esterno della propria organizzazione facendo riferimento alle procedure generali di sicurezza e consulenza governative e di altri network. Potrebbe inoltre essere necessario un supporto adeguato alle procedure e ai requisiti specifici della conservazione digitale.

Le rigorose procedure di sicurezza dovranno:

1. Garantire la conformità con tutti i requisiti legali e normativi.

2. Proteggere i materiali digitali da modifiche involontarie o intenzionali.

3. Fornire una traccia di controllo per soddisfare i requisiti di responsabilità.

4. Agire da deterrente per potenziali violazioni della sicurezza interna.

5. Proteggere l'autenticità delle risorse digitali.

6. Proteggere contro furto o smarrimento.

Molti tipi di risorse digitali selezionate per la conservazione a lungo termine possono contenere informazioni riservate e sensibili che devono essere protette per garantire che utenti non autorizzati non possano accedervi. In molti casi vi possono essere obblighi legali o normativi per l'organizzazione. Tali risorse devono essere gestite in conformità con le procedure di sicurezza delle informazioni dell'organizzazione per proteggere dalle violazioni della sicurezza. La ISO 27001 descrive il modo in cui le procedure di sicurezza possono essere codificate e monitorate (ISO, 2013a). La ISO 27002 fornisce le linee guida sull'implementazione delle 
procedure di sicurezza conformi alla ISO 27001 (ISO, 2013b). Le organizzazioni conformi possono essere accreditate e validate esternamente. In alcuni casi, anche le procedure di sicurezza delle informazioni della propria organizzazione potrebbero avere un impatto sull'attività di conservazione digitale e potrebbe essere necessario richiedere il supporto dei team di Information Governance e ICT per facilitare i processi.

I metodi di sicurezza delle informazioni, come la crittografia, aumentano la complessità del processo di conservazione e, se possibile, dovrebbero essere evitati per le copie di archivio. Altri approcci di sicurezza potrebbero quindi dover essere applicati in modo più rigoroso per file sensibili non crittografati; questi potrebbero includere la limitazione dell'accesso ai terminali bloccati in posizioni controllate (locali sicuri) o requisiti di una solida autenticazione utente per l'accesso remoto. Tuttavia, questi approcci alternativi potrebbero non essere sempre sufficienti o fattibili. La crittografia può anche essere presente sui file ricevuti al momento dell'ingest da un depositante, quindi è importante essere consapevoli delle opzioni di sicurezza delle informazioni come la crittografia, la gestione delle chiavi di crittografia e le loro implicazioni per la conservazione digitale.

\section{Tecniche per la protezione delle informazioni}

Diverse tecniche di sicurezza delle informazioni possono essere applicate per proteggere il materiale digitale:

\section{Crittografia}

La crittografia è una tecnica che protegge il materiale digitale convertendolo in una forma codificata. La crittografia può essere applicata a molti livelli, da un singolo file a un intero disco. Esistono molti algoritmi di crittografia, ognuno dei quali codifica le informazioni in modo diverso. Questi richiedono l'uso di una chiave per decodificare i dati e riconvertirli nella loro forma originale. La forza del metodo di crittografia è influenzata dalla dimensione della chiave. Ad esempio, la crittografia a 256 bit sarà più sicura della crittografia a 128 bit.

Si noti che la crittografia è efficace solo quando una terza parte non ha accesso alla chiave di crittografia in uso. Un utente che ha inserito la password per un'unità crittografata e ha lasciato la propria macchina accesa e incustodita offrirà a terzi l'opportunità di accedere ai dati conservati nell'area crittografata, il che potrebbe comportarne il rilascio.

Allo stesso modo, nel corso del tempo, le misure di sicurezza crittografiche (se utilizzate) possono perdere la loro efficacia in un repository: esiste effettivamente "una corsa agli armamenti" tra tecniche crittografiche e i metodi computazionali per aggirarle. Quindi, se utilizzata, la crittografia di un repository deve essere attivamente gestita e aggiornata nel tempo per garantirne la sicurezza.

II materiale digitale crittografato è accessibile nel tempo in un repository solo se l'organizzazione gestisce le sue chiavi. La perdita o la distruzione di queste chiavi renderanno inaccessibili i dati. 


\section{Controllo di accesso}

I controlli di accesso consentono a un amministratore di specificare chi è autorizzato ad accedere alla risorsa digitale e il tipo di accesso consentito (ad esempio sola lettura, scrittura). II Manuale segue i livelli di conservazione della National Digital Stewardship Alliance (NDSA) nel raccomandare quattro livelli su cui la conservazione digitale può essere supportata attraverso il controllo degli accessi. I livelli della NDSA si concentrano principalmente sulla conoscenza di chi ha accesso ai contenuti, su chi può eseguire quali azioni su tali contenuti e sul rispetto di queste restrizioni di accesso (NDSA, 2013):

\begin{tabular}{|l|l|}
\hline Livello NDSA & Attività \\
\hline 1 & $\begin{array}{l}\cdot \text { Identificare chi ha letto, scritto, spostato ed eliminato le autorizzazioni su singoli } \\
\text { file. } \\
\cdot \text { Limitare chi dispone di tali autorizzazioni su singoli file }\end{array}$ \\
\hline 2 & $\cdot$ Restrizioni di accesso ai documenti in relazione al loro contenuto \\
\hline 3 & $\begin{array}{l}\cdot \text { Conservare i log di chi ha eseguito le azioni sui file, comprese le eliminazioni e } \\
\text { le azioni di conservazione }\end{array}$ \\
\hline 4 & $\cdot$ Eseguire il controllo dei log \\
\hline
\end{tabular}

\section{Revisione}

La revisione si riferisce al processo di analisi di una risorsa digitale, all'identificazione di informazioni riservate o sensibili e alla loro rimozione o sostituzione. Le tecniche comuni che vengono applicate includono l'anonimizzazione e la pseudonimizzazione per rimuovere le informazioni di identificazione personale, nonché la pulizia delle informazioni che riguardano l'autore. In relazione ai set di dati, generalmente questa operazione viene eseguita mediante la rimozione di informazioni mantenendo la struttura del registro nella versione rilasciata. Si dovrebbe sempre eseguire la revisione su una copia dell'originale e mai sull'originale stesso.

La maggior parte delle risorse digitali create utilizzando i pacchetti informatici per ufficio, come Microsoft Office, sono archiviate in formati proprietari e codificati in codice binario. I formati binari potrebbero contenere informazioni significative che non vengono visualizzate, e pertanto la loro presenza potrebbe non essere evidente. Possono includere le cronologie delle modifiche, le tracce di controllo o i metadati incorporati, mediante i quali è possibile recuperare le informazioni cancellate, o semplici processi di revisione altrimenti aggirati. I materiali digitali possono essere redatti attraverso una combinazione di cancellazione delle informazioni e conversione in un formato diverso. Alcuni formati, come semplici file di testo ASCII, contengono solo le informazioni visualizzabili. La conversione in questo formato eliminerà quindi qualsiasi informazione che potrebbe essere nascosta in porzioni non visualizzabili di un flusso di bit. 


\section{Risorse}

\section{ENISA. 2013, Cloud Security Incident Reporting}

https://www.enisa.europa.eu/activities/Resilience-and-CIIP/cloud-computing/incident-reportingfor-cloud-computing/

L'Agenzia europea per la sicurezza delle reti e dell'informazione offre delle raccomandazioni sulle modalità con cui i fornitori di servizi cloud e i loro clienti dovrebbero rispondere - e segnalare - violazioni della sicurezza. (38 pagine).

ISO 27001:2013, Information technology - Security techniques - Information security management systems - Requirements. Geneva: International Organization for Standardization

\section{http://www.iso.org/iso/catalogue detail?csnumber $=54534$}

La ISO 27001 descrive il modo in cui le procedure di sicurezza possono essere codificate e monitorate. Le organizzazioni conformi possono essere accreditate e validate esternamente. $\grave{E}$ disponibile un modello per una serie di politiche allineate allo standard. Si noti che si tratta di voci per aiutare nella creazione di politiche, piuttosto che di dichiarazioni programmatiche. Tuttavia, diverse organizzazioni utilizzano simili politiche (23 pagine).

ISO 27002:2013, Information technology - Security techniques - Code of practice for information security controls. Geneva: International Organization for Standardization http://www.iso.org/iso/catalogue detail?csnumber $=54533$

La ISO 27002 fornisce le linee guida sull'implementazione delle procedure di sicurezza conformi alla ISO 27001. (80 pagine)

ISO 27799:2008, Health informatics - Information security management in health using ISO/IEC 27002. Geneva: International Organization for Standardization http://www.iso.org/iso/catalogue detail?csnumber=41298

La ISO 27799 fornisce consulenza specifica sull'attuazione delle ISO 27002 e 27001 nel settore sanitario. (58 pagine)

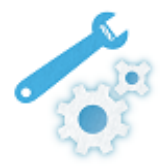

Cabinet Office, 2009, HMG IA Standard No. 1 - Technical Risk Assessment http://www.cesg.gov.uk/publications/Documents/is1 risk assessment.pdf

Una discussione dettagliata e uno standard destinati ai Risk Manager e ai professionisti della sicurezza delle informazioni del Regno Unito, i quali sono responsabili dell'identificazione, della 
valutazione e del trattamento dei rischi tecnici per i sistemi e i servizi che gestiscono, archiviano ed elaborano le informazioni digitali del governo. (114 pagine).

\section{Redaction toolkit (TNA 2011)}

http://www.nationalarchives.gov.uk/documents/information-

\section{management/redaction strumentikit.pdf}

Questo toolkit della TNA è stato prodotto nel 2011 per fornire indicazioni sulla modifica di materiale esente dalle informazioni detenute da enti pubblici. Si riferisce ai principi generali dei record su qualsiasi supporto, ma contiene una breve sezione che riguarda specificatamente $\mathrm{i}$ documenti elettronici e una guida dettagliata sui metodi per la revisione sicura di documenti elettronici di tutti i tipi. (21 pagine).

\section{BitCurator}

http://wiki.bitcurator.net/index.php?title=Main Page

BitCurator è una suite open source di strumenti di analisi forense e di analisi dei dati digitali open per aiutare le istituzioni in possesso di materiali nativi digitali. Alcune parti/sezioni del set di strumenti aiutano a individuare le informazioni private e sensibili su supporto digitale e preparano i materiali per l'accesso del pubblico.

WWW

\section{Information Commissioners Office (ICO): Information security (Principle 7)}

https://ico.org.uk/for-organisations/guide-to-data-protection/principle-7-security/

II sito web dell'ICO fornisce una guida sulla segnalazione di violazioni della sicurezza e sull'uso dell'IT. Per coloro che lavorano in organizzazioni che rientrano nella giurisdizione dell'ICO, è essenziale conoscere le raccomandazioni contenute in questa guida per iniziare a collaborare con i colleghi dell'ICT e della Information Governance, poiché dovranno essere certi che il lavoro può essere svolto in conformità con le raccomandazioni dell'ICO.

\section{Access to the Secure Lab}

http://ukdataservice.ac.uk/get-data/how-to-access/accesssecurelab

Numerose fonti di microdati riservati e sensibili diventano disponibili attraverso datalabs in tutto il Regno Unito. Questi dati sono ritenuti potenzialmente identificabili e sono accessibili solo tramite una struttura di datalab (al contrario del download). Inoltre, ai ricercatori viene chiesto di soddisfare una serie di requisiti applicativi aggiuntivi. Alcuni di questi dati sono accessibili tramite il Secure Lab del Servizio dati del Regno Unito e questa pagina fornisce delle utili descrizioni e l'accesso ad accordi con gli utenti interessati. 


\section{Casi di Studio}

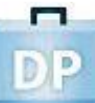

Opening access to administrative data for evaluating public services: The case of the Justice Data Lab

http://evi.sagepub.com/content/21/2/232.full.pdf+html

II Justice Data Lab, un'unità all'interno di un ambiente sicuro con competenze valutative e statistiche, ha consentito ai fornitori di programmi, volti a ridurre la reiterazione del reato, di ottenere prove su come l'impatto dei loro interventi differisse da quello di un gruppo di confronto. Questo articolo analizza lo sviluppo del Justice Data Lab, le sfide metodologiche e di altro tipo affrontate e le esperienze delle organizzazioni di utenti. L'articolo evidenzia le implicazioni per lo sviluppo futuro dei Data Lab e l'uso di dati amministrativi per la valutazione dei servizi pubblici. (16 pagine).

\section{UK Data Service: Data Security}

http://ukdataservice.ac.uk/manage-data/store/security.aspx

Questa pagina web riassume il modo in cui l'archivio dati del Regno Unito gestisce la sicurezza dei dati in suo possesso. La sicurezza dei dati potrebbe essere necessaria per proteggere i diritti di proprietà intellettuale, gli interessi commerciali o per proteggere le informazioni sensibili. Le disposizioni devono essere proporzionate alla natura dei dati e ai rischi connessi. L'attenzione alla sicurezza è necessaria anche quando i dati devono essere distrutti.

\section{Riferimenti}

NDSA, 2013. The NDSA Levels of Digital Preservation: An Explanation and Uses, version 1 (2013). Disponibile al seguente link:

http://www.digitalpreservation.gov/ndsa/working groups/documents/NDSA Levels Archiving 2013.pdf

ISO, 2013a. ISO 27001:2013 - Information technology - Security techniques - Information security management systems - Requirements. Geneva: International Organization for Standardization. Disponibile al link: http://www.iso.org/iso/catalogue detail?csnumber=54534

ISO, 2013b. ISO 27002:2013 - Information technology - Security techniques - Code of practice for information security controls. Geneva: International Organization for Standardization. Disponibile al seguente link: http://www.iso.org/iso/catalogue detail?csnumber=54533 


\section{Servizi Cloud}

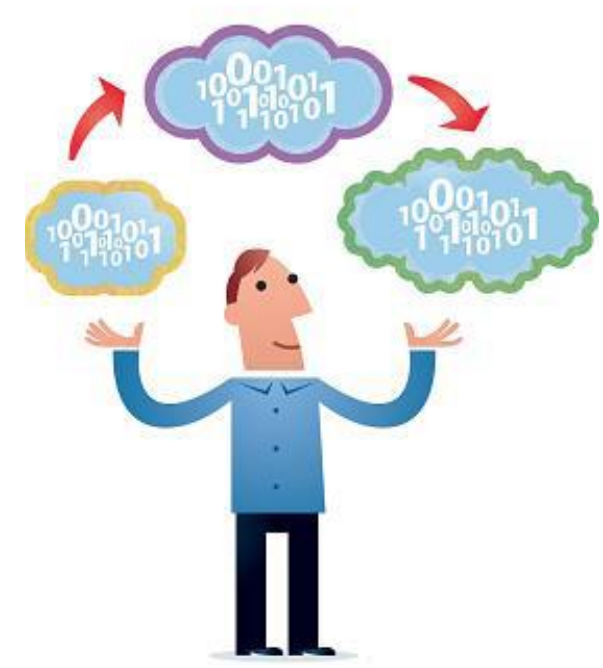

Illustrazione di Jørgen Stamp digitalbevaring.dk CC BY 2.5 Denmark

\section{Che cos'è il cloud computing?}

Cloud Computing è un termine che comprende diversi casi d'uso e modelli di implementazione. In sostanza, un "cloud" informatico è un ampio pool condiviso di risorse informatiche tra cui il servizio di archiviazione dei dati. È possibile ricercare, se necessario, maggiori prestazioni di elaborazione direttamente dal pool senza richiedere un impegno da parte del team IT, e ciò permette di ridurre i costi e anche, in modo sostanziale, il tempo necessario per iniziare a utilizzare nuove risorse di elaborazione. La maggior parte di questi "cloud" sono gestiti su Internet da aziende ben note come Amazon e Google. Alcune organizzazioni più grandi hanno anche tratto vantaggio dall'utilizzo di cloud privati all'interno dei propri data center, dove iniziano ad applicarsi simili economie di scala.

Le caratteristiche generalmente accettate di un tipico servizio cloud possono essere:

- Disponibili "su richiesta", senza la necessità di lunghi processi di acquisizione e configurazione;

- Disponibili su reti standard come Internet, senza requisiti speciali per reti, protocolli o hardware specifici o proprietari;

- In grado di offrire una capacità aggiuntiva all'aumentare della domanda, e minore al diminuire della domanda ("elastica");

- In grado di fatturare ai clienti per lo spazio di archiviazione che utilizzano.

\section{Cloud computing e conservazione digitale}

II cloud computing può offrire diversi vantaggi, tra cui:

- La flessibilità del cloud consente di testare i fornitori di servizi emergenti in tempi relativamente rapidi e a basso costo. Esistono già alcune attività pilota con questi servizi cloud e delle opportunità di apprendimento condiviso in tutta la comunità; 
- Attualmente si hanno una maggiore flessibilità e più opzioni nella distribuzione dei servizi di archiviazione cloud e quindi una maggiore attinenza ai repository di archiviazione rispetto agli anni precedenti (si veda cloud pubblici, comunitari, privati e ibridi);

- Vi sono potenziali risparmi sui costi grazie ad acquisizioni e economie di scala più semplici, in particolare per piccoli repository. Questi sono importanti in un momento di pressioni finanziarie;

- I servizi cloud possono fornire la replica automatizzata in più sedi, essenziali per la pianificazione del recupero aziendale e l'accesso all'archiviazione digitale professionalmente gestita; inoltre, gli specialisti possono dare accesso anche ad altri strumenti, procedure, flussi di lavoro e contratti di assistenza dedicati, adattati alle esigenze della conservazione digitale.

\section{Modelli di servizi cloud e fornitori di servizi}

Esistono quattro diversi modelli di servizi cloud:

- Pubblico: servizi commerciali ospitati in grandi data center in tutto il mondo, accessibili tramite reti pubbliche a chiunque disponga dei mezzi per pagare.

- Privato: le organizzazioni di grandi dimensioni creano il proprio cloud virtualizzando grandi set di server fisici all'interno dei propri data center.

- Ibrido: combina aspetti del cloud pubblico e privato, in genere per gestire ampie fluttuazioni nella domanda o per soddisfare diversi requisiti di sicurezza.

- Community: dal punto di vista dell'architettura, potrebbe somigliare a un servizio cloud pubblico, ma ottimizzato per un determinato gruppo di utenti ai quali è riservato l'accesso.

Allo stato attuale esistono due classi di provider di servizi cloud: generalisti che offrono servizi di archiviazione cloud (Amazon, Rackspace, Google, ecc.), e specialistici che rispondono ad aggiuntivi e specifici requisiti e funzioni di conservazione digitale (si veda Risorse e Casi di

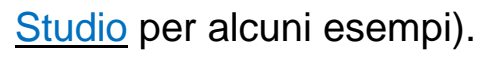

\section{Vantaggi}

- I servizi cloud possono fornire una replica semplice e automatizzata in più sedi, l'accesso all'archiviazione digitale gestita professionalmente e il controllo dell'integrità. Di conseguenza, la conservazione dei bit delle informazioni digitali può essere almeno altrettanto buona se non migliore di quanto si possa conseguire a livello locale;

- Gli archivi possono dare anche accesso a strumenti, procedure, flussi di lavoro e contratti di assistenza dedicati, su misura per i requisiti di conservazione digitale tramite fornitori specializzati;

- Vi sono potenziali risparmi sui costi grazie ad acquisizioni e economie di scala più semplici, in particolare per archivi più piccoli;

- La flessibilità del cloud consente test e casi pilota relativamente rapidi ed economici da parte dei provider;

Esiste una maggiore flessibilità e più opzioni nella distribuzione dei servizi cloud e quindi una maggiore importanza data agli gli archivi rispetto agli anni precedenti. In particolare, 
le implementazioni del cloud privato o del cloud ibrido possono rispondere a problemi di sicurezza relativi all'archiviazione di materiale più sensibile, forse considerato non idoneo per il cloud pubblico;

- È possibile attuare strategie di uscita per rispondere alle preoccupazioni archivistiche sulla stabilità e longevità del fornitore 0 altri rischi relativi al cambiamento. Ad esempio, la sincronizzazione del contenuto tra due fornitori di servizi cloud o un cloud esterno con memoria interna locale o concordare una copia di sicurezza detenuta in modo indipendente da parti terze fidate;

- Esistono già alcune attività pilota con questi servizi cloud e opportunità di apprendimento condiviso in tutta la comunità.

\section{Svantaggi}

- Il cloud è progettato per la sua flessibilità e per i rapidi cambiamenti. Gli archivi tuttavia sono a lungo termine. I contratti di archiviazione e di servizi cloud richiedono un'attenta gestione nel tempo per soddisfare le esigenze di archiviazione. I dati conservati negli archivi devono essere conservati e accessibili al di là della durata commerciale di qualsiasi attuale tecnologia o fornitore di servizi;

- Il cloud può essere più economico, ma spesso richiede che le organizzazioni pensino in modo diverso al modo in cui vengono gestiti i budget. Esistono anche diverse competenze per i fornitori di servizi IT e per la gestione dei contratti che possono comportare costi di riqualificazione o di reclutamento;

- I servizi cloud pubblici tendono a fatturare ogni mese sulla base di quanto effettivamente consumato. Di conseguenza, può essere difficile preventivare il budget o prevedere con precisione la quantità di dati che possono essere caricati, archiviati o scaricati (tuttavia alcuni fornitori possono fatturare un abbonamento annuale in base al volume);

- Come per qualsiasi forma di esternalizzazione, è importante che gli archivi siano molto attenti alla valutazione e al controllo dei rischi dell'archiviazione cloud. Garantire che siano soddisfatti tutti i requisiti e gli obblighi legali relativi ai diritti di terzi relativi ai dati da archiviare. Questi ultimi possono riguardare la gestione, la conservazione o l'accesso e possono essere stati collocati negli archivi e nelle loro organizzazioni produttrici dai loro donatori e finanziatori tramite contratti e accordi o tramite leggi governative;

- L'utilizzo dei servizi cloud prevedrà che gli archivi prendano in considerazione le domande relative al copyright, tra cui: chi detiene attualmente il copyright; se possono essere necessarie autorizzazioni di licenza aggiuntive; quali autorizzazioni saranno necessarie al fornitore cloud per fornire il servizio; se il fornitore cloud è in grado di utilizzare i dati per i propri scopi; e quale parte deterrà i diritti su tutti i dati o opere create dai dati originali;

- L'utilizzo di servizi cloud può sollevare problemi di sicurezza dei dati nel caso in cui essi siano"dati personali" (ad esempio dati che consentono l'identificazione di un individuo), tra cui la determinazione della responsabilità per la protezione dei dati e l'audit dei fornitori, nonché della posizione del trattamento e della misura in cui i rischi derivanti dall'automazione della prestazione di servizi possono essere affrontati per contratto; 
- Gli elementi legali della relazione tra un archivio e un fornitore di servizi cloud (ad esempio in termini di contratti di servizio e accordi sul livello di servizio) devono essere ben definiti e soddisfare i propri requisiti. Questo può essere difficile poiché molti fornitori di servizi cloud hanno contratti di servizio standard per raggiungere i prezzi dei prodotti e hanno una flessibilità limitata in termini di negoziazione;

- È necessario prevedere esplicitamente le strategie di uscita predefinite e le procedure efficaci di collaudo, monitoraggio e audit.

\section{Conclusioni}

Il termine "cloud" può comprendere una vasta gamma di modelli di implementazione per i servizi di conservazione digitale. Si può apprendere molto dalle organizzazioni che hanno già sperimentato o si sono spostate verso l'uso del cloud. Ad esempio, diversi archivi sono stati in grado di affrontare gli interrogativi più diffusi sui servizi cloud e trovare modi per integrare con successo l'archiviazione cloud nelle loro attività di conservazione digitale. Altri utilizzano servizi basati su cloud per tutte o parte delle altre funzioni di conservazione digitale come la pianificazione della conservazione. In definitiva, procurare servizi cloud è simile a procurarsi qualsiasi IT. I rischi vanno gestiti e affrontati allo stesso modo di qualsiasi altro aspetto della propria infrastruttura IT.

\section{Risorse}

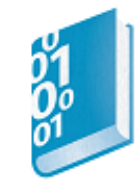

The National Archives Guidance on Cloud Storage and Digital Preservation (2nd Edition 2015)

http://www.nationalarchives.gov.uk/documents/CloudStorage-Guidance March-2015.pdf

Questa guida esamina il modo in cui si sta sviluppando l'archiviazione cloud nella conservazione digitale, le opzioni emergenti e le buone pratiche, insieme ai requisiti e agli standard che gli archivi dovrebbero considerare. Vengono fornite sezioni incentrate su servizi, questioni legali e cinque casi di studio collegati. Sono incluse inoltre, fonti di ulteriore consulenza e orientamento. (39 pagine).

Aitken, B, McCann, P, McHugh, A and Miller, K, 2012, Digital Curation and the Cloud, DCC http://www.jisc.ac.uk/media/7/C/1/\%7B7C1A1FD7-44B4-4951-85A8-

FC2C4CEB1564\%7DCuration-in-the-Cloud master final.pdf

Questo report del 2012 si è concentrato sull'uso dei servizi cloud per la gestione dei dati della ricerca. Fornisce alcune definizioni di cloud computing ed ha esaminato una serie di approcci cloud aperti agli istituti di istruzione superiore nel 2012. (30 pagine).

Anderson. S, 2014, Feet on The Ground: A Practical Approach to The Cloud Nine Things to Consider When Assessing Cloud Storage, AV Preserve

http://www.avpreserve.com/wp-content/uploads/2014/02/AssessingCloudStorage.pdf 
Un white paper sui servizi cloud, suddiviso in nove argomenti e domande. Sono disponibili i profili dei fornitori sui suddetti nove argomenti. (7 pagine).

\section{A. Brown, C. Fryer, 'Achieving Sustainable Digital Preservation in the Cloud'} http://www.girona.cat/web/ica2014/ponents/textos/id87.pdf

Questo documento descrive il modo in cui il Parlamento sta utilizzando il cloud come parte della sua infrastruttura di repository digitale. 2004 (10 pagine).

\section{Digital Preservation Specialist Cloud Service Providers \\ ArchivesDirect \\ http://archivesdirect.org}

ArchivesDirect presenta un'istanza ospitata da Archivematica con archiviazione tramite DuraCloud in archiviazione sicura e replicata di Amazon S3 e Amazon Glacier.

\section{Arkivum}

http://arkivum.com

Arkivum's Archive as a Service offre un servizio completamente gestito e sicuro per la conservazione dei dati a lungo termine con accesso online e una garanzia di integrità dei dati che fa parte del suo Accordo sul livello di servizio e supportato da un'assicurazione mondiale.

\section{DuraCloud}

\section{http://www.duracloud.org}

DuraCloud è un servizio gestito da DuraSpace. Fornisce supporto e strumenti che copiano automaticamente il contenuto su diversi provider di archiviazione cloud e garantisce che tutte le copie del contenuto rimangano sincronizzate. Si veda anche ArchivesDirect per il servizio congiunto con Archivematica.

\section{Preservica}

\section{http://preservica.com/edition/cloud-edition/}

Preservica Cloud Edition è una piattaforma di conservazione digitale conforme a OAIS e completamente ospitata su cloud che include anche l'accesso/rilevamento pubblico per consentire la condivisione in sicurezza dell'archivio o della collezione.

WWW

\section{David Rosethal's blog}

http://blog.dshr.org/

Contiene numerosi post sull'economia del cloud computing. 
The National Archives case study: Archives \& Records Council Wales Digital Preservation Working Group

http://www.nationalarchives.gov.uk/documents/Cloud-Storage-casestudy Wales 2015.pdf

Questo caso di studio descrive l'esperienza di un gruppo di lavoro intersettoriale di archivi gallesi che collaborano per testare una vasta gamma di sistemi e distribuzioni di servizi in una proof of concept per l'archiviazione cloud. Spiega il contesto organizzativo, la diversa natura dei loro requisiti, gli approcci per la conservazione digitale e la loro esperienza nella selezione, implementazione e test della conservazione digitale nel cloud. Il caso di studio ha esaminato il software Archivematica open source con Microsoft Azure di Windows; Archivematica con CloudSigma; Preservica Cloud Edition e ha iniziato a testare Archivematica con Arkivum 100. Gennaio 2015 (10 pagine).

The National Archives case study: Tate Gallery

http://www.nationalarchives.gov.uk/documents/Cloud-Storage-

casestudy Tate Gallery 2015.pdf

Questo caso di studio illustra l'esperienza di sviluppo di un archivio digitale condiviso per le quattro sedi fisiche della Tate, alimentate da un sistema di archiviazione commerciale di Arkivum. Spiega il contesto organizzativo, la natura dei loro requisiti e gli approcci per la conservazione digitale e la loro logica per la scelta della soluzione on-premise di Arkivum, "Arkivum / OnSite", preferendo qualsiasi offerta basata su cloud. Si conclude con le lezioni chiave apprese e si discutono i piani per lo sviluppo futuro. Gennaio 2015 (7 pagine).

The National Archives case study: Dorset History Centre

http://www.nationalarchives.gov.uk/documents/Cloud-Storage-case-

study Dorset $2015 \% 281 \% 29$.pdf

Questo caso di studio riguarda il Dorset History Center, un servizio di archivio del governo locale. Descrive il contesto organizzativo dell'archivio, la natura dei suoi requisiti e gli approcci di conservazione digitale, il suo progetto pilota di due anni che utilizza Preservica Cloud Edition (un servizio di conservazione digitale basato su cloud), l'infrastruttura tecnica dell'archivio, il caso aziendale e il finanziamento del progetto pilota. Si conclude con le lezioni chiave apprese e i piani futuri. Gennaio 2015 (9 pagine).

The National Archives case study: Parliamentary Archives

http://www.nationalarchives.gov.uk/documents/Cloud-Storage-

casestudy Parliament 2015.pdf

Questo caso di studio riguarda gli Archivi parlamentari e la loro esperienza di acquisizione tramite il framework G-Cloud. Per una maggiore forma resilienza/strategia di uscita, hanno selezionato due fornitori di servizi cloud con diverse infrastrutture di archiviazione sottostanti. Questo è un esempio di un archivio che utilizza un set ibrido di soluzioni di archiviazione 
(parzialmente cloud pubblico e parzialmente installato localmente) per la conservazione digitale poiché l'archivio ha un sistema di conservazione installato localmente (Preservica Enterprise Edition) integrato con cloud e archiviazione locale e sta archiviando materiale sensibile localmente, non nel cloud. Gennaio 2015 (6 pagine).

\section{The National Archives case study: Bodleian Library, University of Oxford}

http://www.nationalarchives.gov.uk/documents/Cloud-storage-casestudy Oxford 2015.pdf

Questo caso di studio copre la Bodleian Library e I'Università di Oxford, e la fornitura di un'infrastruttura locale "cloud privato" per le sue collezioni digitali tra cui libri digitalizzati, immagini e multimedia, dati della ricerca e cataloghi. Spiega il contesto organizzativo, la sua natura dei requisiti e gli approcci per la conservazione digitale, i suoi servizi di archiviazione, l'infrastruttura tecnica, il caso aziendale e il finanziamento. Si conclude con le lezioni chiave apprese e i piani futuri. Gennaio 2015 (6 pagine).

\section{King's College London Kindura Project}

http://link.springer.com/article/10.1186\%2F2192-113X-2-13

II progetto Kindura guidato dal King's College di Londra e finanziato da JISC ha cercato di pilotare l'uso di un cloud ibrido per la gestione dei dati della ricerca. Ha usato DuraCloud per mediare tra risorse di archiviazione o di calcolo fornite da servizi cloud esterni, servizi condivisi o servizi interni. Esiste un caso di studio precedente preparato da JISC, ma è stato collegato tramite link a un articolo più recente sul progetto a libero accesso.

\section{University of Illinois Archives 2011 evaluation of Archivematica}

http://e-records.chrisprom.com/evaluating-open-source-digital-preservation-systems-a-case-

\section{study-2/}

Angela Jordan descrive una valutazione del 2011 dell'Archivio di Archivematica dell'Università dell'llinois, un sistema di conservazione digitale open source conforme al Modello di riferimento OAIS. Poiché Archivematica era in fase alfa, lavorare con questo sistema era un modo per esplorare ciò che il sistema offriva in relazione alle esigenze degli Archivi dell'Università, oltre a fornire input agli sviluppatori mentre continuavano a perfezionare il software per il rilascio di produzione. 


\section{Informatica forense}

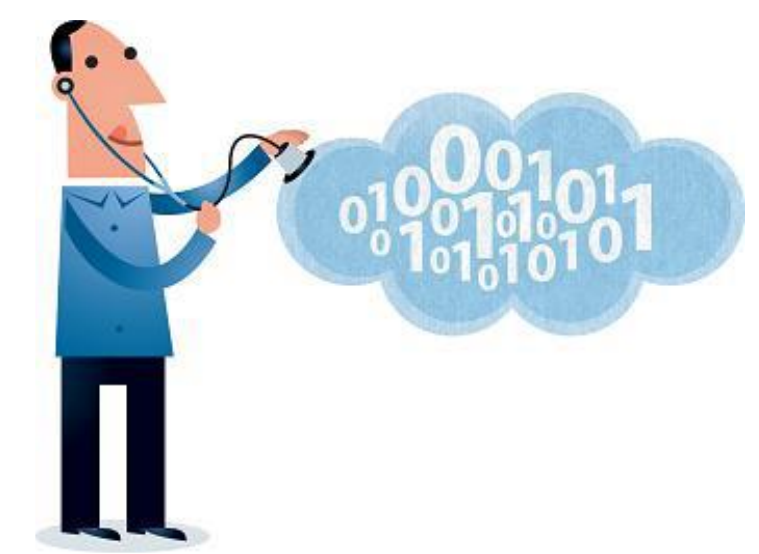

Illustrazion di Jørgen Stamp digitalbevaring.dk CC BY 2.5 Denmark

\section{Introduzione}

Molte persone associano l'informatica forense principalmente all'indagine di illeciti. Tuttavia, negli ultimi anni si è anche affermata come una promettente fonte di strumenti e approcci per facilitare la conservazione e la curation digitali, in particolare per proteggere e indagare le prove del passato.

I repository istituzionali e i professionisti con responsabilità sugli archivi privati e altre raccolte digitali possono trarre vantaggio dalla scienza forense nel trattare questioni relative all'autenticità, responsabilità e accessibilità digitali. Le informazioni personali digitali devono essere gestite con la dovuta sensibilità e sicurezza, proteggendo, nel contempo, e in modo dimostrabile, il loro valore probatorio.

La tecnologia forense consente di: identificare i problemi di privacy; stabilire una catena di custodia per quanto riguarda la provenienza; utilizzare la protezione da scrittura per l'acquisizione e il trasferimento; rilevare falsificazioni o manipolazioni. Può estrarre metadati e contenuti pertinenti; consentire l'indicizzazione e la ricerca efficienti da parte dei curatori; facilitare il controllo di audit e i privilegi di accesso granulari. Le capacità avanzate promettono un'automazione via via più efficace nella gestione di volumi sempre più elevati di informazioni digitali personali. Con le giuste politiche in atto, l'uso giudizioso delle tecnologie forensi continuerà a offrire modelli teorici, soluzioni pratiche e approfondimenti analitici.

\section{La scienza forense nella pratica}

Esistono tre principi di base ed essenziali nella scienza forense digitale: che l'evidenza viene acquisita senza che essa venga alterata; che ciò si può dimostrare; che l'analisi è condotta in modo responsabile e ripetibile. I processi della scienza forense digitale, I'hardware e i software sono stati progettati per garantire la conformità a questi requisiti.

La sicurezza delle informazioni è fondamentale. II blocco dei diritti di scrittura assicura che le informazioni vengano acquisite senza essere alterate, mentre le catene di custodia in termini di 
gestione delle prove, controllo dei processi, audit delle informazioni, firme digitali e filigrana proteggono le prove storiche da alterazioni future e provenienza incerta.

La revisione selettiva, l'anonimizzazione e la crittografia, il controllo della sandbox del malware e altri meccanismi per la sicurezza sono necessari per garantire che la privacy sia completamente protetta e che la perdita involontaria di informazioni venga impedita. I computer di famiglia, i dispositivi portatili e i servizi cloud in condivisione contengono all'interno numerose informazioni personali e, di conseguenza, comportano problemi relativi alla privacy. Gli archivisti digitali e i professionisti forensi condividono la necessità di gestire le informazioni personali in modo responsabile.

L'attuale enfasi sull'automazione nella ricerca forense digitale è particolarmente significativa per la cura del patrimonio culturale, nell'ambito del quale questa capacità riveste una crescente importanza in un universo digitale che continua sempre più ad espandersi. Ricerche attuali mirano a gestire grandi volumi in modo efficiente ed efficace utilizzando una varietà di tecniche analitiche. L'elaborazione parallela, ad esempio, attraverso GPU (Graphics Processing Unit) appositamente progettate e l'elaborazione ad alte prestazioni posso assistere attività che prevedono risorse di elaborazione ad alta intensità come la ricerca e l'indicizzazione complete, il filtraggio e l'hashing, la cancellazione sicura, il mining, la fusione e la visualizzazione.

Particolarmente degno di nota per la conservazione e la curation digitale è il modo in cui l'informatica forense pone l'attenzione sull'elemento multimediale digitale nel suo insieme - che è in genere l'immagine del "disco forense", il file che rappresenta tutto sul disco originale.

\section{Tecnologie forensi}

Le tecnologie forensi variano notevolmente in termini di capacità, costi e complessità. Alcune attrezzature sono costose, ma alcune sono gratuite. Alcune tecniche sono molto semplici da usare, altre devono essere applicate con grande cura e raffinatezza. II Consorzio BitCurator ha rappresentato un passaggio importante che ha riunito una comunità di utenti di archivistica che usano strumenti forensi digitali open source (Lee et al, 2014). Esiste un set sempre più ricco di strumenti forensi open source che sono gratuiti da ottenere e da utilizzare, il che è particolarmente significativo per gli archivisti, BitCurator. Questi strumenti permettono una descrizione accurata dei dettagli relativi alla scienza forense digitale e può essere utilizzata per confrontare e verificare trasversalmente le uscite di strumenti commerciali o di altri strumenti open source.

Gli archivisti digitali e gli specialisti forensi condividono l'esigenza comune di monitorare e comprendere come la tecnologia viene utilizzata per creare, archiviare e gestire le informazioni digitali. Inoltre, vi è la necessità reciproca di gestire tali informazioni in modo responsabile in conformità agli standard pertinenti e alle buone pratiche. Nuove tecniche forensi stanno promuovendo la gestione delle informazioni digitali da dispositivi mobili, reti, dati in tempo reale su computer remoti, supporti flash, macchine virtuali, servizi cloud e fonti crittografate. L'uso della crittografia sta iniziando a presentare sfide significative per la conservazione digitale. Non 
è solo una questione di decodifica, ma di identificazione innanzitutto della crittografia. L'informatica forense offre alcune soluzioni.

La metodologia forense e archivistica deve conservare la capacità sia di interpretare retrospettivamente gli eventi rappresentati su dispositivi digitali, sia di reagire rapidamente al panorama digitale che muta in linea con la rapida istituzione di politiche, procedure e strutture certificabili e responsabili. II ritmo del cambiamento ha anche implicazioni per la formazione permanente di curatori e archivisti e ci sono corsi di informatica forense approvati da istituzioni archivistiche, accademiche e di conservazione.

\section{Conclusione}

In conclusione, ci sono alcune sfide significative per il patrimonio culturale e gli archivi, ma la prospettiva forense è senza dubbio tra le fonti più promettenti di approfondimenti e soluzioni. Allo stesso modo, la scienza forense digitale può beneficiare dei progressi compiuti nella cura e nella conservazione delle informazioni digitali.

Questa breve panoramica è basata su alcuni estratti del Report sulla tecnologia di conservazione digitale su Digital Forensics and Preservation (John, 2012) con materiale aggiuntivo gentilmente fornito da Jeremy Leighton John, l'autore del report. Si veda Risorse e casi di studio per ulteriori indicazioni dettagliate ed esempi.

\section{Risorse}

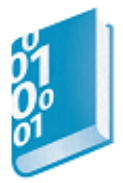

\section{Digital forensics and preservation DPC technology watch report}

http://dx.doi.org/10.7207/twr12-03

Questo report della DPC del 2012 offre un'ampia panoramica dell'informatica forense, con alcuni suggerimenti su risorse e strumenti che possono andare a beneficio del patrimonio culturale e in particolare della cura degli archivi digitali privati (60 pagine).

\section{Digital forensics and born-digital content in cultural heritage collections}

\section{http://www.clir.org/pubs/reports/pub149/pub149.pdf/view}

La presente relazione CLIR introduce il campo della scienza forense digitale nel settore dei beni culturali ed esplora alcuni punti di convergenza tra gli interessi delle persone incaricate della raccolta e della conservazione dei materiali del patrimonio culturale digitale nativo e quelle incaricate della raccolta e della conservazione delle prove legali (93 pagine). 


\section{Archivematica}

https://www.archivematica.org/wiki/Main Page

Archivematica è un sistema di conservazione digitale open source che si è occupato dell'importazione di immagini di dischi forensi come parte dei suoi flussi di lavoro e set di strumenti.

WWW

\section{BitCurator}

http://www.bitcurator.net

II sito Web fornisce l'accesso alle informazioni sul BitCurator Consortium (BCC), i progetti e gli strumenti. BitCurator ha sviluppato, impacchettato e documentato strumenti forensi digitali open source per consentire a biblioteche, archivi e musei di estrarre materiali digitali da supporti rimovibili in modo da rappresentare i metadati e garantire l'integrità dei materiali, consentendo agli utenti di dare un senso alle risorse e comprendere il loro contesto e prevedendo la divulgazione involontaria di dati sensibili. II consorzio è un'associazione di appartenenza indipendente guidata dalla comunità che figura come host e centro di supporto amministrativo, utente e comunità per l'ambiente BitCurator.

\section{Forensics wiki}

\section{http://forensicswiki.org/wiki/Main Page}

The Forensics Wiki è un wiki con licenza Creative Commons dedicata alle informazioni sull'informatica forense. Elenca oltre 700 pagine incentrate sugli strumenti e sulle tecniche utilizzate dagli investigatori, documenti e relazioni importanti, persone e organizzazioni coinvolte.

The Invisible Photograph Part 2: Trapped: Andy Warhol's Amiga Experiments http://www.nowseethis.org/invisiblephoto/posts/108

Un team di informatici, archivisti, artisti e curatori si è unito per scoprire le opere digitali perdute di Andy Warhol su un computer Amiga Commodore di 30 anni fa (18 minuti e 52 secondi)

The Invisible Photograph Part 3: Extraterrestrial: The Lunar Orbiter Image Recovery Project

http://www.nowseethis.org/invisiblephoto/posts/384

Come gli "archeologi della tecnologia" del Lunar Orbiter Image Recovery Project hanno recuperato digitalmente le prime fotografie della luna scattate da una serie di sonde spaziali senza pilota negli anni '60. (22 minuti 07 secondi) 
Casi di Studio

\section{Carcanet email project}

http://www.library.manchester.ac.uk/aboutus/projects/carcanet/

Un progetto finanziato da JISC che ha affrontato la sfida di acquisire e preservare l'archivio email di Carcanet Press, una delle principali case editrici di poesia del Regno Unito. È stato vincitore del DPC Preservation Award del 2014 per la salvaguardia dell'eredità digitale. II progetto ha esplorato e adottato diversi strumenti di ediscovery e forensi, in particolare AccessData Forensic Toolkit (FTK), Paraben's Email Examiner e Fookes Software Aid4Mail eDiscovery. Un report finale del progetto riassume il lavoro (Baker, 2014).

\section{Riferimenti}

John, J. L., 2012. Digital Forensics and Preservation. DPC Technology Watch Report 12-03 November 2012. Disponibile al seguente link: http://dx.doi.org/10.7207/twr12-03

Lee, C. A., Olsen, P., Chassanoff, A., Woods, K., Kirschenbaum, M. \& Misra, S., 2014. From Code to Community: Building and Sustaining BitCurator through Community Engagement. BitCurator White Paper 30 September 2014. Disponibile al seguente link: http://www.bitcurator.net/wp-content/uploads/2014/11/code-to-community.pdf 


\section{Identificatori persistenti}

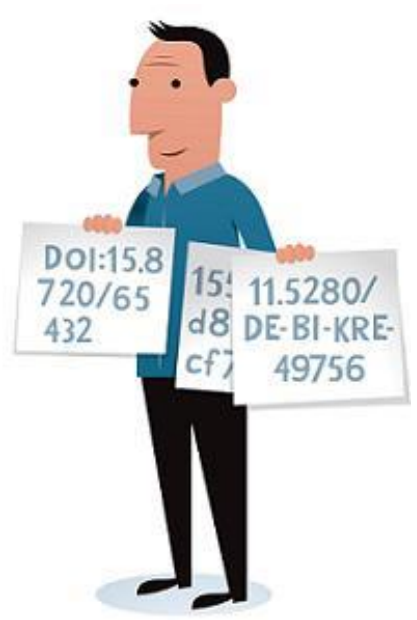

Illustrazione di Jørgen Stamp digitalbevaring.dk CC BY 2.5 Denmark

\section{Introduzione}

Questa sezione fornisce una guida sull'uso di identificatori persistenti per oggetti digitali e per la conservazione digitale. Esistono altri tipi di schemi di identificazione persistenti, ad esempio per privati o istituzioni.

Un identificatore persistente è un riferimento duraturo a una risorsa digitale. In genere ha due componenti: un identificatore univoco e un servizio che individua la risorsa nel tempo anche quando cambia posizione. II primo aiuta a garantire la provenienza di una risorsa digitale (che è ciò che pretende di essere), mentre il secondo assicurerà che l'identificatore si risolva nella posizione corrente corretta.

Gli identificatori persistenti hanno l'obiettivo, quindi, di risolvere il problema della persistenza dell'accesso alle risorse citate, in particolare nella letteratura accademica. Troppo spesso, gli indirizzi web (link) non riescono a portare alla risorsa referenziata che ci si aspetta. Questo può essere dovuto a ragioni tecnologiche, come il fallimento del server, sebbene i guasti provocati dall'uomo siano quelli più comuni. Le organizzazioni trasferiscono le riviste a nuovi editori, riorganizzano i loro siti Web o perdono interesse per i contenuti più vecchi, portando a collegamenti interrotti quando si tenta di accedere a una risorsa. Questo potrebbe essere scoraggiante per gli utenti, ma le conseguenze possono essere gravi se la risorsa collegata attraverso link è essenziale per motivi legali, medici o scientifici.

Gli identificatori persistenti possono anche essere utilizzati "dietro le quinte" allinterno di un repository per gestire alcune sfide nella catalogazione e nella descrizione, o nel fornire controllo intellettuale e accesso a materiali digitali nativi. 


\section{Schemi}

Poiché il problema della persistenza di un identificatore viene creato dall'uomo, la soluzione di identificatori persistenti deve coinvolgere anche persone e servizi, non solo tecnologie. Esistono diversi schemi di identificazione persistenti e tutti richiedono l'intervento umano per mantenere i propri sistemi di risoluzione. I principali schemi di identificatori persistenti attualmente in uso sono di seguito riportati.

\section{Digital Object Identifier (DOI)}

I DOI sono identificatori digitali per oggetti (digitali, fisici o astratti) che possono essere assegnati dalle organizzazioni appartenenti a una delle agenzie di registrazione DOI; i due più noti sono CrossRef, per articoli di riviste e alcune altre pubblicazioni accademiche, e DataCite per una vasta gamma di oggetti dati. Oltre all'identificatore di oggetto, il DOI dispone di un'infrastruttura di sistema per garantire che un URL si risolva nella posizione corretta per quell'oggetto.

\section{Handle}

Gli handle sono identificatori univoci e persistenti per le risorse di Internet, con un registro centrale per la risoluzione degli URL nella posizione corrente. Ogni handle identifica una singola risorsa e l'organizzazione che ha creato o che gestisce in quel momento la risorsa. II Sistema Handle sostiene anche l'infrastruttura tecnica dei DOI, che sono un tipo speciale di handle.

\section{Chiave di risorsa di archivio (ARK)}

ARK è uno schema identificativo ideato dalla California Digital Library (CDL), con l'obiettivo di identificare gli oggetti in modo persistente. Lo schema è stato progettato sulla base del fatto che la persistenza "è puramente una questione di servizio e non è inerente a un oggetto né conferita ad esso da una particolare sintassi di denominazione".

\section{Localizzatore di risorse uniformi persistenti (PURL)}

I PURL sono URL che reindirizzano alla posizione della risorsa Web richiesta utilizzando codici di stato HTTP standard. Un PURL è quindi un indirizzo Web permanente che contiene il comando per reindirizzare a un'altra pagina, che può cambiare nel tempo.

\section{Nome risorsa universale (URN)}

Gli URN sono identificatori persistenti, indipendenti dalla posizione, che consentono l'identificazione della risorsa all'interno di un namespace. L'esistenza di un simile identificatore non implica la disponibilità della risorsa identificata, ma tali URI devono rimanere univoci e persistenti a livello globale, anche quando la risorsa cessa di esistere o diventa non disponibile. Il termine URN ora è in disuso, tranne nel senso molto stretto di uno spazio dei nomi formale per esprimere un identificatore di risorsa uniforme.

\section{Scegliere uno schema di identificazione persistente}

È necessario un contratto sociale per mantenere la persistenza del servizio di risoluzione - da parte dell'organizzazione che ospita la risorsa digitale, di una terza parte fidata o di una 
combinazione delle due. Ogni schema ha i suoi vantaggi e vincoli, ma vale la pena considerare quanto segue quando si decide su una strategia o un approccio di identificativo persistente:

\section{Vantaggi}

- Di fondamentale importanza per aiutare a stabilire l'autenticità di una risorsa.

- Fornisce l'accesso a una risorsa anche se la sua posizione cambia.

- Supera i problemi causati dalla natura temporanea degli URL.

- Consente l'interoperabilità tra le collezioni.

\section{Svantaggi}

- Non esiste un sistema unico accettato da tutti, anche se i DOI sono ben consolidati e ampiamente utilizzati.

- Potrebbero esserci costi per stabilire o utilizzare un servizio.

- Dipendenza dal vincolo al mantenimento continuo del sistema di identificazione permanente.

\section{Conclusioni}

Gli identificatori persistenti devono essere supportati da servizi permanenti e non solo da stringhe univoche di caratteri alfanumerici assegnati a una risorsa digitale. Sono diventati particolarmente importanti per i dati della ricerca e gli articoli su riviste elettroniche (Si veda la

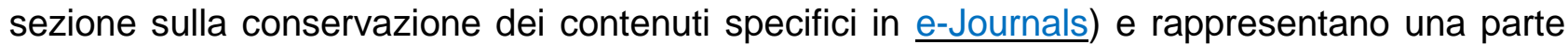
significativa dell'infrastruttura a lungo termine per la conservazione digitale della ricerca. Per questioni relative al link-rot per le pagine Web più generali e le soluzioni che utilizzano archivi web per risolvere questo problema, si consulti la sezione relativa alla conservazione dei contenuti specifici sull'Archiviazione Web.

\section{Risorse}

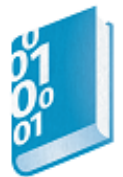

\section{Persistent identifiers - an overview. TWR Technology Watch Review \\ http://www.metadaten-twr.org/2010/10/13/persistent-identifiers-an-overview/}

Questo articolo di Juha Hakala (2010) descrive cinque sistemi di identificazione persistenti (ARK, DOI, PURL, URN e XRI) e confronta la loro funzionalità con gli URI. L'obiettivo è fornire una panoramica, non dare alcun tipo di classificazione di questi sistemi.

\section{Preservation, trust and continuing access for e-Journals DPC technology watch report http://dx.doi.org/10.7207/twr13-04}

Questo report del 2013 di Neil Beagrie discute gli sviluppi e le questioni attuali che le biblioteche, gli editori, gli intermediari e i fornitori di servizi stanno affrontando nel settore della conservazione digitale, della fiducia e dell'accesso continuo alle riviste elettroniche. Comprende lezioni e raccomandazioni generiche sull'esternalizzazione, sulla fiducia dell'interesse per la più 
ampia comunità della conservazione digitale e copre importanti questioni legali, economiche e di servizio nonché relative alla tecnologia. (49 pagine).

\section{Persistent Identifiers in the Publication and Citation of Scientific Data http://www.iza.org/en/papers/5090 28102009.pdf}

Presentazione di Jens Klump, Centro di ricerca tedesco per le geoscienze (GFZ) sul progetto DFG STD-DOI, che illustra in dettaglio il background e il ragionamento alla base di DataCite. 2009. (47 pagine).

\section{Briefing Paper: Persistent Identifiers}

http://www.dcc.ac.uk/resources/briefing-papers/introduction-curation/persistent-identifiers

Questo articolo del 2006 di Joy Davidson discute come i progressi nella definizione della natura e dei requisiti funzionali dei sistemi di identificazione siano ostacolati dalla mancanza di un accordo condiviso su ciò che gli identificatori dovrebbero effettivamente fare; fornire semplicemente un nome univoco globale o locale per una risorsa digitale 0 analogica 0 incorporare servizi associati come la risoluzione e l'associazione di metadati. L'applicazione e la manutenzione degli identificatori costituisce solo una parte di una strategia globale di conservazione digitale; al fine di offrire garanzie di persistenza a lungo o breve termine necessitano/si necessita di impegno istituzionale e ruoli e responsabilità chiaramente definiti. (2 pagine)

\section{ARK}

http://www.collib.org/services/uc3/arkspec.pdf

\section{CrossRef}

http://www.crossref.org

DataCite

http://www.datacite.org

DOI

http://www.doi.org/

\section{Handle}

http://www.handle.net/

\section{Perma.CC}

https://perma.cc/about 
PURL

https://purl.org/docs/index.html

URN

http://tools.ietf.org/html//rfc3986

Casi di Studio

D $P$

DCC case study: Assigning digital object identifiers to research data at the University of Bristol

http://www.dcc.ac.uk/resources/persistent-identifiers

L'Università di Bristol gestisce un repository di dati della ricerca dedicato come parte del loro Research Data Service. Sta utilizzando il servizio DataCite della British Library per assegnare identificatori di oggetti digitali (DOI) a ricerche di set di dati al fine di fornire identificatori univoci e perpetui per i dati, per consentire una facile citazione e rilevabilità. II Bristol Research Data Service fornisce una guida su come utilizzare gli identificatori per citare i dati e sta sviluppando politiche appropriate per monitorare l'utilizzo. 2004. (4 pagine).

\section{Links that Last}

http://www.dpconline.org/events/previous-events/925-links-that-last

Questa giornata di briefing DPC nel luglio 2012 ha introdotto gli argomenti degli identificatori persistenti e dei dati collegati e ha discusso delle implicazioni pratiche di entrambi gli approcci alla conservazione digitale. Ha considerato la fattibilità dei servizi che offrono gli identificatori persistenti e ciò che questi garantiscono nel contesto della conservazione; ha esaminato $i$ recenti sviluppi nei linked data, considerando come tali set di dati potrebbero essere conservati; e, introducendo questi due argomenti paralleli, ha continuato a considerare se entrambi gli approcci possono essere fattibili per creare una nuova classe di dati collegati in maniera solida. Diverse presentazioni, inclusi casi di studio, sono collegate al programma provvisorio. 


\section{Conservazione di Contenuti Specifici}


Illustrazione di Jørgen Stamp digitalbevaring.dk CC BY 2.5 Denmark

\section{A chi è rivolto?}

Responsabile dell'area operativa (DigCurV Manager Lens) e personale (DigCurV Practitioner Lens) addetto ai repository, editori e altri creatori di dati, fornitori di servizi terzi.

\section{Requisiti minimi di conoscenza}

Da principiante a intermedio

\section{Obiettivo}

- Creare delle sinergie con i report della serie DPC Tech Watch. I report forniscono un'analisi approfondita a livello avanzato in aree specifiche della conservazione dei contenuti (ad es., e-mail). I report possono essere citati o usati per richiamare dei casi di studio presenti nel Manuale.

- Da sviluppare per facilitare la manutenzione, economicità e sostenibilità a lungo termine da parte della DPC tramite aggiornamenti e aggiunte alla serie Tech Watch.

- Fornire una breve panoramica e dei casi di studio, adatti a utenti principianti o di livello intermedio, sui problemi della conservazione digitale per specifici tipi di contenuto trattati dai DPC Technology Watch Reports. Attualmente sono disponibili tre tipi di contenuto: riviste on line, immagini in movimento e suono e archiviazione web. In futuro si intende aggiungere altre tipologie.

\section{Riutilizzare queste informazioni}

È possibile riutilizzare questo materiale in lingua inglese (ad eccezione dei loghi) necessariamente con dei riconoscimenti gratuiti in qualsiasi formato o con qualsiasi mezzo. Per i dettagli completi sulle autorizzazioni e riconoscimenti per il riutilizzo si veda la sezione $\underline{\text { Come }}$ usare il Manuale.

Per autorizzazioni alla traduzione in altre lingue si contatti per e-mail: handbook@dpconline.org 
Si prega di usare questa citazione per il Manuale: Digital Preservation Handbook, 2nd Edition, https://www.dpconline.org/handbook, Digital Preservation Coalition @ 2015. 


\section{Riviste on line}

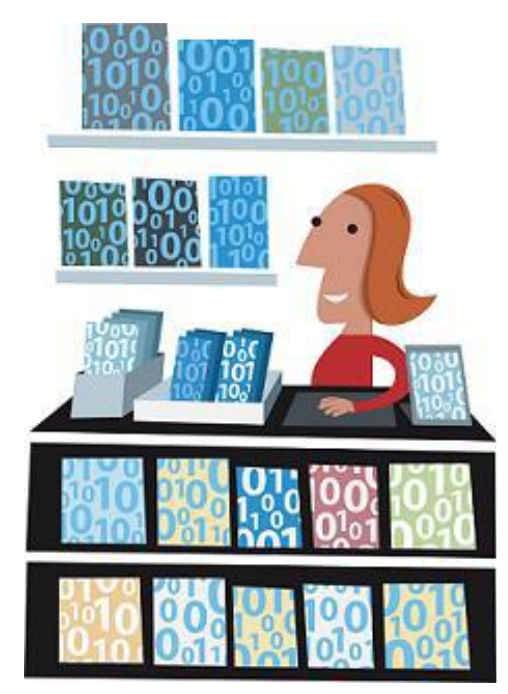

Illustrazione di Jørgen Stamp digitalbevaring.dk CC BY 2.5 Denmark

\section{Panoramica}

Questo caso di studio, per utenti principianti e di livello intermedio, fornisce una breve panoramica della conservazione di riviste on line ripresa dal "DPC Technology Watch Report Preservation, Trust and Continuing Access for e-Journals" con alcuni aggiornamenti e aggiunte da parte dell'autore. Comprende due "mini casi di studio" insieme a delle brevi sintesi dei principali servizi e soluzioni. II report è consigliato ai lettori che necessitano di un briefing di livello più avanzato sull'argomento e sugli aspetti pratici. Tratta diverse questioni e attività in modo più approfondito con ulteriori approfondimenti e consigli (Beagrie, 2013).

\section{Introduzione}

La conservazione digitale e la certezza di avere accesso continuo ai contenuti digitali in futuro sono diventati sempre più importanti per le biblioteche di ricerca poiché le riviste e gli articoli pubblicati sono passati dai formati cartacei a quelli elettronici. Anche i modelli e le relazioni commerciali editoriali tradizionali hanno subito importanti trasformazioni a seguito di tale cambiamento.

Uno degli importanti cambiamenti avvenuti riguarda il passaggio osservato dall'acquistare e possedere (e conservare) localmente una rivista cartacea (con una ridondanza multipla di copie tra le biblioteche), all'abbonamento (licenza) ad una rivista elettronica tenuta sulle piattaforme degli editori che spesso hanno sede a livello internazionale in altre giurisdizioni.

Parallelamente, è avvenuto un graduale spostamento verso gli articoli in riviste elettroniche open access che cerca di rimuovere i costi di abbonamento per l'accesso. Le riviste in abbonamento, le riviste open access e le riviste ibride (una combinazione di articoli in accesso aperto e in abbonamento in una stessa rivista oppure una rivista che permette l'accesso dopo 
un determinato periodo di tempo) forniscono un panorama complesso per la conservazione di e l'accesso a lungo termine a riviste elettroniche.

Il panorama delle riviste on line continua ad evolvere man mano che la stessa pubblicazione on line inizia a passare da contenuti statici a contenuti dinamici e che nelle principali discipline aumenta l'importanza delle informazioni e del materiale supplementare collegato agli articoli.

Tutti questi cambiamenti, a loro volta, hanno reso la conservazione delle riviste elettroniche più impegnativa, più internazionale e dipendente da altri, e hanno sollevato questioni legate alla fiducia. La fiducia in questo contesto non riguarda solo la tecnologia per la conservazione, ma la negoziazione dei diritti (e la conservazione di una documentazione per un uso futuro) insieme alla disponibilità di informazioni trasparenti su ciò che viene archiviato, su come viene conservato e su come e quando è possibile accedervi.

Ciò rende le riviste elettroniche una delle aree più dinamiche e stimolanti della conservazione digitale, in particolare in termini di modelli di business e meccanismi di fiducia per servizi di conservazione condivisi o di provenienza esterna.

\section{Servizi e soluzioni}

È importante comprendere le implicazioni significative che i diversi requisiti che si applicano alle riviste elettroniche - insieme alla terminologia - hanno per la conservazione e l'accesso: in particolare la distinzione tra accesso continuo e conservazione a lungo termine, poiché queste differenze portano a diversi tipi di servizi per l'archiviazione delle riviste elettroniche.

- L'accesso continuo (a volte chiamato anche post-cancellazione o accesso perpetuo) si applica solo alle riviste in abbonamento e garantisce un accesso a lungo termine per i loro abbonati;

- La conservazione a lungo termine si applica sia al contenuto aperto che a quello in abbonamento.

I principali servizi e soluzioni di conservazione e accesso continuo disponibili per le riviste elettroniche sono i seguenti:

Keepers Registry

II Keepers Registry è un servizio JISC per fornire informazioni facilmente accessibili sull'inclusione di riviste elettroniche nei servizi di conservazione e per evidenziare quelle riviste elettroniche per le quali non esistono accordi di archiviazione. EDINA, un centro dati nazionale con sede presso I'Università di Edimburgo, ha sviluppato il servizio insieme al suo partner di progetto, il Centro Internazionale ISSN di Parigi.

Legal and voluntary deposit in copyright libraries

II ruolo di una biblioteca nazionale è garantire che il patrimonio pubblicato nel suo paese sia preservato e reso accessibile. In molti paesi il deposito legale è un veicolo importante per 
raggiungere questo obiettivo. Generalmente, si protende verso un'estensione del deposito legale dall'ambiente cartaceo per includere anche le riviste elettroniche e altre pubblicazioni elettroniche. La legislazione sui depositi legali (o simili accordi volontari sui depositi) normalmente coinvolge quelle riviste elettroniche in abbonamento considerate parte del patrimonio nazionale pubblicato in quel paese. Per proteggere gli interessi commerciali dell'editore, limita anche l'accesso off-site al materiale elettronico conservato per un periodo di tempo sostanziale. In genere ciò significa che una raccolta di depositi legali nazionali non copre la gamma internazionale di riviste elettroniche in abbonamento concesse in licenza da altre biblioteche e dai loro utenti e non soddisfa i loro requisiti per i diritti di "accesso perpetuo".

\section{CLOCKSS}

CLOCKSS (Controlled LOCKSS) è una collaborazione senza fini di lucro tra biblioteche ed editori. È un dark archive basato sul software LOCKSS (vedere la sezione seguente su LOCKSS) in cui un numero limitato di biblioteche assume un ruolo di archiviazione per conto di una comunità più ampia. Assicura alle biblioteche che la rivista elettronica e gli altri contenuti a cui sono abbonate saranno conservati a lungo termine. Viene descritta come una "rete LOCKSS privata".

KB e-Depot

La Koninklijke Bibliotheek (KB) è la biblioteca nazionale dei Paesi Bassi e gestisce l'e-Depot. Ha preso la decisione politica di archiviare le riviste che rientrano nel suo mandato nazionale insieme ad una serie di riviste elettroniche (compresi i titoli ad accesso aperto nell'elenco delle riviste ad accesso aperto) pubblicate oltre i suoi confini. Attualmente l'e-Depot non prevede l'accesso continuo post-cancellazione da parte dei licenziatari del contenuto. Generalmente, l'accesso dell'utente finale è limitato alla lettura in loco presso la KB solo per motivi di ricerca individuale e l'accesso online è negato. Tuttavia, l'accesso completo online è concesso alle pubblicazioni degli editori open-access.

\section{LOCKSS}

LOCKSS (Lots of Copies Keep Stuff Safe) fornisce alle biblioteche strumenti e supporto open source in modo che possano prendere in custodia locale una vasta gamma di materiali, inclusi abbonamenti e risorse accademiche ad accesso aperto (libri, riviste, ecc.). I lettori accedono ai contenuti conservati da LOCKSS ogni volta che (e per qualsiasi motivo) il materiale non può essere visualizzato sui server dell'editore (o dell'intermediario). Questo approccio distribuito mira a garantire che vi siano copie a sufficienza per salvaguardare il contenuto nonostante eventuali disastri che potrebbero colpire le singole istituzioni LOCKSS.

Portico

Portico è progettato specificamente come un servizio di parti terze per la letteratura accademica pubblicata in formato elettronico e fornisce tre servizi di conservazione specifici per riviste on line, e-book e collezioni storiche digitalizzate. Garantisce alle biblioteche che le riviste elettroniche e gli altri contenuti a cui sono abbonati saranno conservati a lungo termine. Portico fornisce l'accesso solo alle riviste elettroniche conservate dopo specifici eventi scatenanti (trigger events). Inoltre, Portico può fungere anche da potenziale meccanismo per l'accesso post-cancellazione.

Consortial hosting

Un piccolo numero di consorzi regionali organizza e fornisce i propri servizi di hosting per l'accesso e la conservazione delle riviste elettroniche. Esempi degni di nota sono OhioLink, gestito dalla 
Ohio Library and Information Network, e lo Scholars Portal, gestito dall'Ontario Council of University Libraries.

Caso di studio 1: la rivista on line o i suoi numeri precedenti non sono più disponibili presso l'editore

Questo è uno scenario verosimile e si verifica quando gli editori si fondono o cambiano i loro modelli di business, quando gli editori più grandi riesaminano e adeguano il loro portfolio di titoli, oppure quando le società spostano i contratti di pubblicazione delle loro riviste da un editore all'altro. I titoli delle riviste vengono talvolta scambiati tra editori, il che potrebbe significare che l'accesso al numero precedente non è più supportato dal precedente proprietario.

L'iniziativa UKSG Transfer Code of Practice ha prodotto un Codice di pratica volto ad alleviare i problemi creati quando i titoli delle riviste vengono spostati da un editore ad un altro. Di rilievo sono i seguenti paragrafi contenuti nella versione 3 del codice (UKSG, 2014):

- L'editore che trasferisce avviserà l'editore che riceve di tutti gli accordi di conservazione esistenti per la rivista.

- L'editore che trasferisce deve garantire l'accesso continuo ai propri abbonati laddove abbia concesso diritti di accesso perpetuo, anche se questo cesserà di ospitare la versione online della rivista dopo la data di trasferimento effettiva.

- L'editore trasferente o ricevente o entrambi potrebbero adempiere agli obblighi di accesso perpetuo. II Codice non specifica intenzionalmente i mezzi per ottenere tale accesso, ma attribuisce all'editore trasferente la responsabilità di garantire che gli abbonati ai quali ha concesso i diritti di accesso perpetuo continuino ad avere accesso dopo il trasferimento.

- L'editore trasferente farà ogni ragionevole sforzo per comunicare le informazioni di trasferimento della rivista alla quale sono stati concessi diritti di accesso perpetuo nell'ambito di un accordo di licenza/Big Deal, a meno che i diritti di archiviazione non rimarranno all'editore trasferente.

- I diritti di accesso perpetuo a contenuti precedentemente pubblicati concessi agli abbonati devono essere garantiti.

- L'editore trasferente o ricevente o entrambi potrebbero adempiere agli obblighi di accesso perpetuo.

- L'editore ricevente continuerà le disposizioni di conservazione esistenti o equivalenti per la rivista dopo la data effettiva di trasferimento.

- L'editore ricevente non rimuoverà il contenuto precedentemente depositato nella conservazione degli archivi, anche se non continuerà a depositare i contenuti negli archivi.

La decisione dell'editore Sage di non offrire più la sua pubblicazione Graft ha fornito un esempio reale di accesso attivato da tre soluzioni di archiviazione: Portico, KB e-Depot e CLOCKSS. In questo caso, tutti sono stati in grado di continuare ad offrire l'accesso ai numeri in loro possesso, sia attraverso l'accesso aperto (CLOCKSS e KB e-Depot) sia come servizio ai membri (Portico). 
Sebbene non si possa garantire che l'archivio includerà tutti i numeri arretrati del titolo (come con Graft), la partecipazione a una soluzione di archiviazione che copre almeno alcuni numeri ridurrà significativamente il rischio di interruzione della continuità del servizio.

Caso di studio 2: riviste on line in biblioteca, accesso perpetuo e rimozione della

\section{stampa}

Questo caso di studio è stato pubblicato per la prima volta da JISC come parte del lavoro finanziato nel suo programma di conservazione digitale ed è stato incorporato nel Tech Watch Report. È stato adattato per l'uso nel Manuale.

Questo caso di studio presenta rispetto agli altri delle problematiche legate all'attuazione di alcuni dei potenziali risparmi sui costi grazie alle riviste elettroniche, in particolare risparmi riferiti allo spazio. Sempre più spesso le biblioteche accademiche investono molto in riviste elettroniche che prendono il posto delle copie cartacee esistenti. Per le biblioteche che devono affrontare questioni di spazio, una soluzione è quella di rimuovere le copie a stampa che si sovrappongono con quelle elettroniche.

Il caso di studio si concentra sul lavoro presso la Biblioteca dell'Imperial College di Londra che consiste nel fornire un database e un kit di strumenti al personale che prende tali decisioni di de-selezione (Cooper e Norris, 2007). Imperial ha stabilito tre criteri per determinare la sostenibilità delle proprie riviste elettroniche e per la rimozione di quelle a stampa. II loro accesso elettronico è stato classificato come sostenibile quando si applicava almeno uno dei seguenti principi:

- Imperial aveva diritti di accesso perpetuo al contenuto, via web. I diritti di accesso perpetuo di Imperial non erano affatto completi come avrebbero voluto; hanno stimato una copertura di meno del $50 \%$ del loro contenuto. Inoltre, alcune delle loro licenze hanno specificato un metodo di consegna non idoneo per l'accesso successivo alla cessazione. Poiché non supportavano più i CD-ROM in rete e non disponevano delle risorse per caricare i contenuti della rivista in locale, hanno considerato una rivista sostenibile solo se l'accesso perpetuo è fornito via web.

- La rivista era ad accesso aperto per tutti gli anni o per determinati anni. Le riviste ibride ad accesso aperto non sono state incluse in questa categoria, poiché il progetto non era interessato alla sostenibilità a livello di articolo. Trovare riviste ad accesso aperto che soddisfacessero i loro criteri si è rivelato più difficile del previsto. II principale ostacolo era la loro necessità di sicurezza sulla permanenza dell'accesso aperto. Sebbene le Dichiarazioni di Bethesda e Berlino sull'accesso aperto includano l'accesso perpetuo nelle loro definizioni, Imperial ha scoperto che non tutte le "riviste ad accesso aperto" soddisfacevano questo criterio di permanenza.

- Il contenuto si trovava in uno dei servizi di fiducia di Imperial come JSTOR, l'archivio digitale ACM o un archivio finanziato da JISC. Imperial ha osservato che tra i loro tre criteri di sostenibilità, questo, che copre servizi che non offrivano diritti di accesso 
perpetuo, era il più difficile da definire. I servizi che rientrano in questa categoria condividevano tutte e due le caratteristiche: la prima era una comprovata esperienza di stabilità, cioè avevano dimostrato continuità nei titoli da un anno all'altro per tutto il tempo in cui erano abbonati; la seconda era rappresentata dalla sua storica convenienza e dal suo rapporto qualità-prezzo.

Ventuno mesi prima del progetto, Imperial aveva identificato 700 metri quadrati di contenuti sostenibili da rimuovere da un sito e aveva pianificato l'attività di de-selezione per altri siti. Sebbene fossero ancora agli inizi, sentivano che i loro criteri di sostenibilità sembravano funzionare. L'unico contenuto sostenibile che avevano perso era rappresentato da quattro riviste dello stesso editore, e si accingevano ad affrontare questa perdita. La banca dati sui diritti d'accesso che avevano creato per il progetto si è rivelata vantaggiosa; senza di essa non avrebbero saputo che i contenuti sui quali avevano i diritti di accesso perpetuo erano andati persi.

\section{Conclusioni}

L'accesso continuo e la conservazione delle riviste elettroniche hanno comportato l'avvio di iniziative nell'organizzazione della collaborazione multi-istituzionale, nello sviluppo di servizi di parti terze e nella fiducia nell'accesso e nella conservazione a lungo termine tra le diverse parti interessate. Le questioni affrontate vanno ben oltre la tecnologia, e gli sviluppi legali, economici e di servizio sono ugualmente fondamentali per il successo. Rimangono molte sfide nell'archiviazione delle riviste elettroniche, ma diversi sono stati i successi significativi raggiunti e le lezioni apprese che possono essere di interesse per la più ampia comunità della conservazione digitale, nonché per biblioteche ed editori.

\section{Risorse}

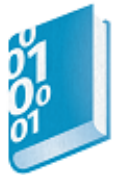

Preservation, Trust and Continuing Access for e-Journals, DPC Technology Watch Report 13-04 September 2013

http://dx.doi.org/10.7207/twr13-04

Questa relazione presenta gli sviluppi e le questioni attuali che le biblioteche, gli editori, gli intermediari e i fornitori di servizi stanno affrontando nel settore della conservazione digitale, della fiducia e dell'accesso continuo relativo alle riviste elettroniche. Include anche lezioni generiche e raccomandazioni sull'esternalizzazione e sulla fiducia apprese in questo campo rivolte alla comunità più ampia della conservazione digitale. Non si concentra esclusivamente sulla tecnologia e tratta importanti questioni legali, economiche e di servizio (43 pagine).

To bin or not to bin? Deselecting print back-runs available electronically at Imperial College London Library

\section{https://spiral.imperial.ac.uk/handle/10044/1/503}


Sempre più spesso le biblioteche accademiche investono molto in riviste elettroniche che duplicano i numeri a stampa. Per le biblioteche che affrontano questioni di spazio, una soluzione al loro problema è quella di rimuovere le copie a stampa che si sovrappongono ai numeri elettronici. Questo articolo del 2007 di R. Cooper e D. Norris descrive il lavoro presso la Biblioteca dell'Imperial College di Londra per fornire un kit di strumenti al personale che prende tali decisioni di de-selezione.

\section{UKSG, 2014 Transfer Code of Practice: Version 3.0 March 2014}

http://www.uksg.org/Transfer/Code

II Codice di condotta per i trasferimenti promuove una serie di standard che si applicano ogni volta che una rivista viene trasferita da un editore o piattaforma di pubblicazione a un altro. Gli editori che si iscrivono al Codice e lo applicano nella pratica sono considerati "conformi al trasferimento". Vista come codice volontario di buone pratiche per i partecipanti del settore, il Codice di condotta per i trasferimenti non sostituisce i termini contrattuali, i diritti di proprietà intellettuale o il mercato competitivo tra gli editori.

\section{CLOCKSS}

http://www.clockss.org

\section{KB e-Depot}

http://www.kb.nl/en/organisation/research-expertise/long-term-usability-of-digitalresources/information-for-international-publishers

\section{LOCKSS}

http://www.lockss.org

\section{Portico}

http://www.portico.org

\section{Ohio Link}

http://www.ohiolink.edu

\section{Scholars Portal}

http://www.ocul.on.ca/node/135

Keepers Registry

http://thekeepers.org 


\section{Riferimenti}

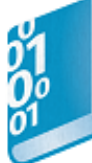

Beagrie, N., 2013. Preservation, Trust and Continuing Access for e-Journals DPC Technology Watch Report 13-04 September 2013. Disponibile al seguente link: http://dx.doi.org/10.7207/twr13-04

Cooper, R. and Norris, D., 2007. To bin or not to bin? Deselecting print back-runs available electronically at Imperial College London Library, Serials 20 (3), 208-214. Disponibile al seguente link: https://spiral.imperial.ac.uk/handle/10044/1/503

UKSG, 2014. Transfer Code of Practice: Version 3.0 March 2014. Disponibile al seguente link: http://www.uksg.org/Transfer/Code 


\section{Immagini in movimento e suono}

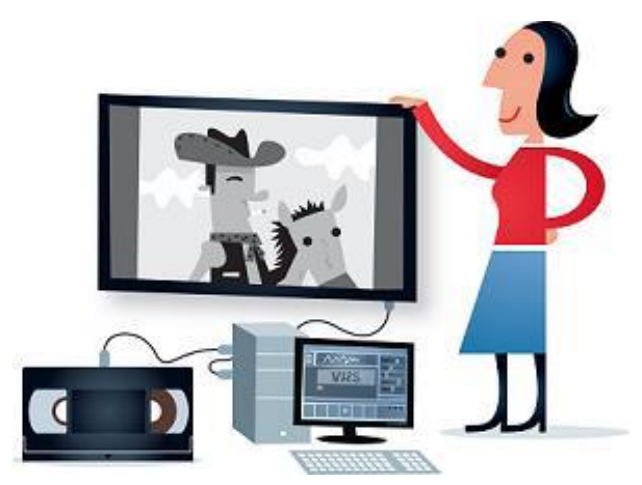

Illustrazione di Jørgen Stamp digitalbevaring.dk CC BY 2.5 Denmark

\section{Panoramica}

Questo caso di studio fornisce una breve panoramica, per utenti principianti e di livello intermedio, tratta dal Report DPC Technology Watch Report on Preserving Moving Picture and Sound. Sono inclusi cinque "mini casi di studio" di collezioni britanniche che hanno avviato progetti di conservazione e accesso a contenuti audio e a immagini in movimento. II report stesso fornisce una "immersione profonda" che discute una serie di questioni più generali e di pratiche in modo approfondito con letture e consigli aggiuntivi (Wright, 2012). Si consiglia ai lettori che necessitano di un briefing di livello più avanzato sull'argomento e sull'attività pratica.

\section{Introduzione}

II dominio audiovisivo è unico in quanto la digitalizzazione è una routine fondamentale per la conservazione. La digitalizzazione audiovisiva per la conservazione è così pervasiva che le due parole sono state usate in modo intercambiabile. L'audio e il video necessitano di digitalizzazione per la sopravvivenza stessa dei loro contenuti, a causa dell'obsolescenza delle apparecchiature di riproduzione e del decadimento e del danneggiamento di oggetti fisici, sia analogici che digitali. II problema della tecnologia di base per le raccolte di immagini in movimento e per il suono è la necessità di digitalizzare tutti i contenuti che si trovano attualmente sugli scaffali. II film sugli scaffali può essere conservato (a meno che non si stia già deteriorando), ma necessita comunque di un processo di digitalizzazione per consentirne l'accesso.

Una questione vitale nella conservazione è l'accesso: la motivazione e il finanziamento per la digitalizzazione a puro scopo di conservazione sono difficili, se non impossibili. C'è un grande interesse pubblico, istituzionale ed educativo nella registrazione audiovisiva del ventesimo secolo. Creare l'accesso a quel record rappresenta la chiave per ottenere il supporto necessario per la digitalizzazione e la conservazione del contenuto.

Il panorama delle "immagini in movimento e del suono" è complicato: fisicamente ci sono grandi differenze tra le registrazioni audio, video e cinematografiche. I formati e le apparecchiature di registrazione/riproduzione sono completamente separati; le procedure di digitalizzazione sono 
diverse; i file digitali hanno diversi formati di wrapper e metadati (con alcune sovrapposizioni); e i requisiti di archiviazione differiscono, con i video che richiedono uno spazio di archiviazione del materiale di circa 100 volte al secondo in più rispetto all'audio, e la pellicola digitale ad alta risoluzione che occupa uno spazio di archiviazione di circa 10 volte maggiore rispetto al video. Inoltre, dal punto di vista culturale ed economico, esistono differenze significative nella conservazione e nella cura delle collezioni che provengono da:

- industrie dei media commerciali - musica, cinema e radiodiffusione commerciale in cui la conservazione ha bisogno di una giustificazione commerciale, un caso aziendale;

- enti pubblici - servizi pubblici di radiodiffusione, collezioni accademiche e istituzioni di conservazione del patrimonio come musei nazionali, biblioteche e istituti cinematografici in cui la conservazione ha bisogno di una giustificazione del patrimonio culturale, sebbene sempre più spesso questo settore necessiti di un business case;

- aree tecniche come la medicina, la geologia e la sorveglianza, in cui le registrazioni di immagini o di eventi sismici sono dati non elaborati, conservati come cartelle cliniche o per il ritrattamento; e

altro: una vasta gamma di collezioni indipendenti, che vanno dagli sforzi individuali al materiale raccolto da istituzioni specializzate senza fini di lucro (ad esempio, club di motori a vapore o ricerca etnologica) che non rientrano in nessuna delle categorie di cui sopra, sebbene il loro materiale possa eventualmente essere donato ad una collezione pubblica.

All'interno di questo panorama si trovano diverse tecnologie tra cui l'ingegneria, l'informatica, I'IT, l'archiviazione, la gestione dei media, la gestione delle collezioni museali, la curation, la conservazione, l'accesso, la gestione della conoscenza e la scoperta di risorse.

\section{Sfide tecniche}

Le registrazioni audiovisive sono una realtà surrogata. La tecnologia consente all'ascoltatore e allo spettatore di avere un'idea di come appariva una situazione, ma in realtà cattura solo la sequenza di schemi di luce o pressioni del suono che agiscono sullo strumento di registrazione (videocamera, microfono). Questi schemi (per film) e segnali (per video e audio) sono più simili ai dati che ai manufatti. II requisito di conservazione non è quello di conservare i supporti di registrazione originali, ma conservare i dati e le informazioni recuperati da tali supporti.

Un problema tecnologico chiave è lo spostamento dei contenuti digitali dai supporti (come CD e DVD, videocassette digitali, DAT e minidisc) ai file. Questo "ripping" dal digitale al digitale del contenuto è un'area della conservazione digitale unica nel campo dell'audiovisivo e presenta problemi irrisolti di controllo degli errori nel processo di ripping e trasferimento.

L'ultima area tecnologica riguarda la conservazione digitale del contenuto all'interno dei file risultanti dalla digitalizzazione o dal "ripping" e dei file che sono nativi digitali. Sebbene gran parte di questa conservazione abbia problemi e soluzioni in comune con altri contenuti, esiste un problema specifico nella preservazione della qualità del segnale digitalizzato che è - anche qui - specifico dei contenuti audiovisivi. Gestire la qualità attraverso cicli di codifica lossy, decodifica e riformattazione è una delle maggiori sfide di conservazione digitale per i file audiovisivi. L'altra questione riguarda la gestione dei metadati incorporati. 
Per tre decenni, per quanto riguarda l'audio, e per almeno due decenni per quanto riguarda il video, gli archivi hanno digitalizzato i loro contenuti analogici per la conservazione e l'accesso. Le aree problematiche sono:

- riproduzione corretta degli originali, al fine di ottenere un segnale ottimale da digitalizzare;

- standard: quale livello di compressione, metodo di codifica e formato file utilizzare; ed

- efficienza: digitalizzazione dei materiali analogici esistenti in modo sufficientemente rapido ed economico per far fronte alle dimensioni e all'urgenza del problema.

\section{Le fasi della conservazione di immagini in movimento e suono}

Per la conservazione di audio e di immagini in movimento, è necessario chiarire le seguenti fasi dell'intero processo:

- segnale: l'audio da un microfono, il segnale video che esce da una videocamera. Questi segnali hanno proprietà fisiche (larghezza di banda; gamma dinamica) che possono essere definite e misurate. La qualità di una registrazione e il successo o il fallimento di qualsiasi processo di copia, digitalizzazione o conservazione possono essere ridotti (in gran parte) al modo in cui quel processo mantiene queste due proprietà fisiche del segnale originale;

- registrazione di un segnale su un supporto. Per un secolo, i metodi per catturare un segnale erano legati al tipo di trasmettitore del segnale: un cilindro di cera, una bobina di pellicola o una videocassetta. La tecnologia digitale produce registrazioni indipendenti dai supporti. L'indipendenza dal supporto rappresenta una forma di liberazione: dischi, nastri e pellicole si deteriorano o si danneggiano. Le registrazioni native digitali non sono interessate da questi problemi relativi ai supporti, e da qui nasce la necessità di liberare le registrazioni analogiche attraverso un processo di digitalizzazione;

- digitalizzazione: le registrazioni analogiche possono essere riprodotte e registrate su un nuovo supporto, oppure digitalizzate e così liberate dalla dipendenza del supporto. La digitalizzazione deve garantire che la versione digitale abbia la stessa larghezza di banda e gamma dinamica dell'originale, per catturare la qualità originale; e

- conservazione digitale della rappresentazione digitale di un segnale, cioè la conservazione dei numeri, ma anche la conservazione della tecnologia necessaria per decodificare (render) i numeri. II contenuto audiovisivo presenta un problema particolare. La codifica del segnale può rappresentare il risultato di un compromesso, non catturare effettivamente l'intero segnale, ma piuttosto perdere parte di esso (codifica lossy) per ottenere una rappresentazione più compatta, riducendo così i costi di archiviazione e trasmissione. Purtroppo i codificatori/decodificatori (codec) vanno dismessi e vengono sostituiti da una tecnologia più recente. Anche il formato del file che contiene il segnale codificato, il wrapper, è soggetto a obsolescenza. II fallimento e l'obsolescenza della tecnologia di archiviazione e l'obsolescenza dei metodi di codifica/decodifica e dei formati dei wrapper sono i principali problemi della conservazione digitale dei contenuti audiovisivi. 


\section{Accesso e diritti}

I contenuti audio e le immagini in movimento provenienti dal cinema, dalla radiodiffusione e dall'industria della musica commerciale sono vincolati da problemi di diritti. La musica ha una protezione di copyright per il compositore e per l'oggetto fisico contenente un'esecuzione (il cosiddetto copyright magnetico). Le produzioni cinematografiche sono protette e la musica utilizzata in un film mantiene separate le sue protezioni. La radiodiffusione è ancora più complicata, dal momento che tutte le parti coinvolte in una produzione possono avere diritti di utilizzo futuro a seguito di una o due trasmissioni specificate nei contratti tipici. Questi diritti sono visti come una protezione da parte dei titolari dei diritti, ma sono anche visti come restrizioni all'accesso. La situazione per un'emittente pubblica è particolarmente difficile. II pubblico ritiene che qualsiasi produzione da parte di un'emittente pubblica sia già stata pagata da loro, sia già di proprietà pubblica e dovrebbe essere disponibile per l'accesso pubblico. Purtroppo questo parere non si ritrova nella definizione legale che disciplina un'opera quando diventa di dominio pubblico (di solito determinata dalla data di scadenza del copyright e di altri diritti).

\section{Caso di studio 1: The Open University (OU) Accesso al progetto di risorse video} Questo è un progetto di accesso e riutilizzo. L'obiettivo è quello di digitalizzare (ove necessario) le risorse audiovisive precedentemente create dall'OU e inserirle in un sistema di gestione delle risorse in modo che chi si occupa attualmente delle attività didattiche, e non solo, presso la OU possa trovare e utilizzare tali risorse. La conservazione è un sottoprodotto del progetto, piuttosto che un fine in sé. Questo progetto è un esempio importante della combinazione tra conservazione del contenuto e uso del contenuto, importante per l'ente al fine di mettere a disposizione un bilancio e offrire un vantaggio. II progetto è stato presentato al DPC Briefing Day 'Preserving Digital Sound and Vision'. Sono stati digitalizzati 1.200 videocassette e film e i risultati sono stati inseriti nel repository digitale Fedora. Inoltre, sono state digitalizzate 145.000 pagine di documentazione, fornendo così un contesto ai 1.200 elementi e migliorando il loro riutilizzo. L'interfaccia utente presenta granularità e una misura della durata di navigazione. Nel complesso questo progetto è un ottimo esempio di buone pratiche.

\section{Caso di studio 2: progetto di registrazioni sonore del British Library Archive} Questo è un progetto di conservazione e accesso educativo supportato da JISC che è stato svolto (nella sua fase iniziale) dal 2004 al 2006. Una seconda fase ha aggiunto ulteriore materiale. Quasi 50.000 registrazioni di discorsi, musica e suoni di "ambienti umani e naturali" sono state digitalizzate e messe online. II catalogo online è aperto a tutti e gli istituti di istruzione superiore del Regno Unito autorizzati possono anche ascoltare l'audio. Chiunque può ascoltare 2.000 registrazioni (oppure ognuna di queste frequentando la sala di lettura della British Library a Londra). La differenza di accesso tra gli enti di istruzione e il pubblico in generale è tra le restrizioni che ancora sussistono in materia di accesso aperto ai materiali audiovisivi nelle istituzioni pubbliche. 
Caso di studio 3: progetto PSRE dell'Imperial War Museum

L'Imperial War Museum ha una delle maggiori collezioni cinematografiche del Regno Unito. Colleziona film sin dalla sua fondazione nel 1919, a partire dalle riprese della Grande Guerra che portarono alla fondazione dell'istituzione. II fondo PSRE (Public Sector Research Exploitation) ha assegnato un premio di quasi 1 milione di sterline per la catalogazione, la digitalizzazione e l'accesso online (al catalogo e al filmato). Il progetto si è svolto dal 2006 al 2009 ed è di particolare interesse in quanto è specificamente finalizzato all'utilizzo commerciale di una collezione e a modelli di business sostenibili riguardanti la digitalizzazione e l'accesso al web. II risultato del progetto è un sito Web (http://film.iwmcollections.org.uk/) in cui chiunque può visualizzare i contenuti in bassa qualità; richiamare i documenti, immagini fisse e fotogrammi chiave in una lightbox; riempire un carrello per poi acquistare il contenuto.

Caso di studio 4: British University Film and Video Council - progetto Newsfilm Online

Questo è un altro progetto sponsorizzato da JISC. Per quattro decenni fino al 1960 i cinegiornali proiettati nei cinema rappresentarono il principale mezzo con cui mostrare al pubblico immagini in movimento degli eventi attuali per l'epoca. Il progetto iniziale è durato dal 2004 al 2008. I risultati sono disponibili attraverso un sito Web che, come per il progetto BL Archival Sound Recordings, è aperto alle università e ai college registrati. Il pubblico generale può vedere sia il catalogo completo che un singolo fotogramma chiave per ciascun elemento. Dalla fase originale del progetto, il contenuto è stato incrementato con l'aggiunta delle notizie ITN / Reuters che coprono gli eventi a partire dai decenni successivi al declino dei cinegiornali. Gli articoli del cinegiornale sono brevi: il progetto iniziale ha fornito 3.000 ore di contenuti corrispondenti a60.000 articoli. Inoltre, come nel progetto Open University, anche la documentazione è stata messa online per fornire il contesto e per supportare la ricerca e il recupero: 450.000 pagine di bollettini.

\section{(SHUK)}

Caso di Studio 5: progetto BFI e Regional Film Archives Screen Heritage UK

SHUK è un progetto piuttosto grande ( $£ 22,8$ milioni) e complesso (che coinvolge 12 archivi cinematografici regionali oltre al BFI). Si sono verificate delle complicazioni durante il progetto per via di cambiamenti nella struttura e nel finanziamento del BFI, nonché da un cambio di governo e da una serie di altre questioni. Tuttavia, il progetto ha prodotto risultati importanti:

- conservazione, non digitalizzazione: costruzione di un deposito di 6 milioni di sterline per la conservazione del film;

- digitalizzazione: scanner per pellicole e apparecchiature di archiviazione digitale per gli archivi cinematografici regionali; 
- accesso: cataloghi online dei contenuti degli archivi cinematografici regionali, disponibili al pubblico.

SHUK è stato lanciato il 5 settembre 2011 con una produzione congiunta della BBC BFI, The Reel History of Britain (SHUK, 2011).

\section{Conclusioni}

Il problema tecnologico di base per le raccolte di immagini in movimento e di suoni è la necessità della digitalizzazione di tutti i contenuti che attualmente si trovano sugli scaffali. L'audio e il video hanno bisogno della digitalizzazione per la loro stessa sopravvivenza, a causa dell'obsolescenza e del deterioramento di oggetti fisici, sia analogici che digitali. II film sugli scaffali può essere conservato (a meno che non si stia già deteriorando) ma necessita della digitalizzazione per l'accesso.

II playback per una digitalizzazione di qualità implica la necessità di un recupero ottimale della qualità originale, che richiede attrezzature ed esperienza professionali. II principale ostacolo tecnico è che, per molti formati fisici, le attrezzature necessarie sono in gran parte obsolete, il che significa che le parti, le riparazioni e gli operatori qualificati sono sempre più carenti. La raccomandazione urgente è quella di non aspettare! Le risorse audiovisive devono essere documentate e inserite in un piano di conservazione.

La situazione per il patrimonio sonoro è chiara. Esiste il consenso per quanto riguarda standard di digitalizzazione, codifica, wrapper e metadati e sono tutti ben documentati nello IASA TC-04 (IASA, 2009). L'audio non compresso nel wrapper Broadcast Wave Format (BWF) è ampiamente utilizzato e ben supportato. Non vi è alcun motivo per cui la codifica di base possa mai essere modificata, sebbene il wrapper BWF alla fine possa diventare obsoleto. L'unico problema significativo è l'incapacità di alcune applicazioni audio standard di gestire correttamente i metadati BWF incorporati (ARSC, 2011). Gli archivi devono essere consapevoli del rischio della perdita di metadati incorporati. La situazione per quanto riguarda i video è complessa, ma esiste un piano d'azione di PrestoSpace per guidare nelle scelte sulla digitalizzazione di vari formati obsoleti. Sulla conservazione digitale dei file risultanti vi sono suggerimenti dati dal PrestoCentre e dal JISC Digital Media. Una delle sfide più grandi è creare un registry di applicazioni che funzionino correttamente su metadati incorporati dei video, laddove la diversità risulti significativa. Non esiste un singolo wrapper, uno standard di metadati o uno standard di codifica concordato e il passaggio dalla definizione standard all'alta definizione porta ad un nuovo set di applicazioni, wrapper e codifiche.

Tecnologie emergenti possono migliorare l'audio (acquisizione della distorsione della tonalità e la conseguente rimozione della variazione temporale) e i trasferimenti di video (digitalizzazione diretta del segnale RF dalla testina di lettura) e potrebbero essere utili in quei casi in cui la tecnologia attuale non funziona. Quindi la raccomandazione è di non aspettare che tale tecnologia avanzi e si diffonda ulteriormente. Se ci sono problemi di riproduzione che non possono essere risolti, il formato audio o video originale dovrebbe essere mantenuto in modo tale da poter applicare tale tecnologia avanzata in futuro. 
Il controllo di qualità dei risultati della digitalizzazione rimane un problema per i video. È necessaria un'efficace integrazione della tecnologia di elaborazione del segnale attraverso una verifica manuale al fine di produrre un metodo veramente efficiente di controllo della qualità all'interno di un approccio industriale. II controllo di qualità è ugualmente rilevante per la conservazione digitale: eventuali cambiamenti o migrazioni dovuti all'obsolescenza digitale devono essere controllati per preservare la qualità del segnale. Ancora una volta, un approccio puramente manuale non è scalabile (fino a decine di milioni di ore di contenuti audiovisivi nelle collezioni europee), mentre i sostituti puramente algoritmici utilizzati per "guardare e ascoltare" non hanno mai avuto successo e rimangono un'area in cui sono necessarie ulteriori ricerche.

\section{Risorse}

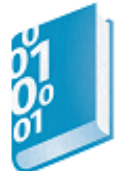

Wright, R., 2012. Preserving Moving Pictures and Sound DPC Technology Watch Report 12-01 March 2012

http://dx.doi.org/10.7207/twr12-02

Questo report è rivolto a chiunque abbia la responsabilità delle raccolte di contenuti audio o di immagini in movimento e un interesse nei confronti della conservazione di tali contenuti. I nuovi contenuti sono nativi digitali, l'audio e il video analogici hanno bisogno della digitalizzazione per sopravvivere e il film richiede la digitalizzazione per l'accesso. Di conseguenza, la conservazione digitale sarà rilevante nel tempo per tutte queste aree. II report si concentra sulla digitalizzazione, la codifica, i formati dei file e i wrapper, l'uso della compressione, l'obsolescenza e su cosa fare riguardo ai particolari problemi della conservazione digitale del suono e delle immagini in movimento (33 pagine).

SHUK, 2011. Screen Heritage UK Marks new Era for Britain's Film Archives

http://www.bfi.org.uk/sites/bfi.org.uk/files/downloads/bfi-press-release-screen-heritage-ukmarks-a-new-era-for-britains-film-archives-2011-09-01.pdf

Comunicato stampa BFI. 8 pagine

IASA 2009 IASA TC-04, Guidelines on the Production and Preservation of Digital Audio Objects (IASA-TC 04 Second edition 2009) Canberra, IASA.

http://www.iasa-web.org/audio-preservation-tc04

Questa è la guida standard alla digitalizzazione dell'audio e le sezioni sui metadati e l'archiviazione digitale sono utili per tutte le forme di supporto digitale.

Casey, M. and Gordon, B., 2007. Best Practices for Audio Preservation. Bloomington, Indiana University Bloomington.

http://www.dlib.indiana.edu/projects/sounddirections/papersPresent/

Un'altra risorsa audio (che include anche una gamma di strumenti software di digitalizzazione) proviene dal progetto Sound Directions delle università di Harvard e Indiana: in gran parte è rilevante anche per la digitalizzazione video. (160 pagine) 
Digital Preservation Coalition Briefing day on Preserving Digital Sound and Vision, April 2011

http://www.dpconline.org/events/details/27-SoundAndVision?xref=26

Questa giornata di briefing della DPC dell'aprile 2011 ha fornito un forum per rivedere e discutere gli ultimi sviluppi nella conservazione del suono e delle immagini digitali. Sette presentazioni associate ai rispettivi link e (inclusa Open University) disponibili per il download.

ARSC Technical Committee, 2011. Study of Embedded Metadata Support in Audio Recording Software. Association of Recorded Sound Collections.

http://www.arsc-audio.org/pdf/ARSC TC MD Study.pdf

Uno studio di supporto sui i metadati incorporati all'interno di diverse applicazioni software di registrazione audio. I risultati destano serie preoccupazioni, in particolare per le comunità di archiviazione e conservazione che fanno affidamento sui metadati incorporati per l'interpretazione e la gestione di file digitali che rappresentano contenuti conservati per il futuro. (21 pagine)

WWW

\section{AVPreserve}

http://www.avpreserve.com/

Società di consulenza per la gestione di informazioni e media con sede negli Stati Uniti. II sito Web offre una gamma di risorse per la conservazione AV.

\section{BUFVC NewsFilm online Project}

http://www.webarchive.org.uk/wayback/archive/20140614061518/http://www.jisc.ac.uk/whatwe do/programmes/digitisation/bufvc.aspx

\section{British Film Institute}

http://www.bfi.org.uk

II British Film Institute può fornire consulenza su film e video: detiene molti video e ha un Curator per la televisione. Tra le sue competenze rientrano la raccolta e la conservazione di materiale proveniente da film e televisione e la prestazione di consulenza tecnica.

\section{British Library Sound Archive}

http://www.bl.uk/nsa

La consulenza tecnica generale sulla conservazione dell'audio è disponibile presso la British Library Sound Archive. II suo compito è la raccolta e la conservazione di tutte le forme di audio e la consulenza tecnica.

\section{Film Archives UK}

http://filmarchives.org.uk 
Raccolta e conservazione di contenuti audiovisivi generali di rilevanza regionale nel Regno Unito.

\section{JISC Digital Media}

http://www.jiscdigitalmedia.ac.uk

Consulenza e formazione su immagini fisse, immagini in movimento e suono. Include i loro InfoKit per i formati dei file digitali, il finanziamento e la sostenibilità della digitalizzazione e la Guida alla digitalizzazione di alto livello per le risorse audiovisive.

\section{PrestoCentre}

http://www.prestocentre.eu

II sito Web fornisce informazioni, risorse e consulenza audiovisive. Di recente, l'accesso è stato esteso a tutte le risorse che adesso sono gratuitamente disponibili per tutti.

\section{Sustaining Consistent Video Presentation}

http://www.tate.org.uk/research/publications/sustaining-consistent-video-presentation

Questo documento tecnico tratta gli approcci per identificare e mitigare i rischi associati nel sostenere la presentazione coerente di file video digitali. Originato da due progetti di ricerca multi-partner - Pericles e Presto4U - il documento è stato commissionato dal Tate Research ed è destinato a coloro che sono attivamente impegnati nella conservazione digitale di video.

\section{JISC 2009 - Archival Sound Recordings Showreel}

https://www.youtube.com/watch?v=KPy9ZaWEHog

Breve video coinvolgente sul progetto di registrazioni sonore dell'archivio della British Library pubblicato il 22 giugno 2009. (6 minuti e 11 secondi).

\section{Altri casi di studio}

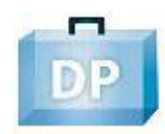

Podcasts in the Archives: Archiving Podcasting Content at the University of Michigan http://files.archivists.org/pubs/CampusCaseStudies/CASE12.pdf

In questo caso di studio del campus della Society of American Archivists, Alexis. A. Antracoli, Università del Michigan, si esaminano le sfide legate allo sviluppo delle buone pratiche e dei flussi di lavoro per l'archiviazione e la conservazione dei contenuti di podcast. Uno dei problemi principali riguardava la definizione di standard per l'ingest, l'archiviazione e l'accesso, in particolare la generazione e l'archiviazione di metadati descrittivi, tecnici e di conservazione. Un'altra sfida riguardava lo sviluppo dell'infrastruttura tecnologica necessaria per supportare un sistema conforme al sistema di informazione sugli archivi aperti (OAIS). 2010. (14 pagine). 


\section{Riferimenti}

ARSC Technical Committee, 2011. Study of Embedded Metadata Support in Audio Recording Software. Association of Recorded Sound Collections. Disponibile al seguente link: http://www.arsc-audio.org/pdf/ARSC TC MD Study.pdf

IASA, 2009. IASA TC-04, Guidelines on the Production and Preservation of Digital Audio Objects, IASA-TC 04 Second edition 2009, Canberra, IASA. Disponibile al seguente link: http://www.iasa-web.org/audio-preservation-tc04

SHUK, 2011. Screen Heritage UK Marks new Era for Britain's Film Archives. Disponibile al seguente link: http://www.bfi.org.uk/sites/bfi.org.uk/files/downloads/bfi-press-release-screenheritage-uk-marks-a-new-era-for-britains-film-archives-2011-09-01.pdf

Wright, R., 2012. Preserving Moving Pictures and Sound DPC Technology Watch Report 12-01 March 2012. Disponibile al seguente link: http://dx.doi.org/10.7207/twr12-02 


\section{Archiviazione web}

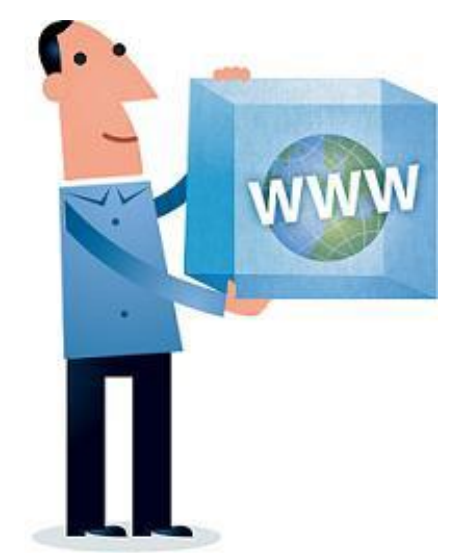

Illustrazione di Jørgen Stamp digitalbevaring.dk CC BY 2.5 Denmark

\section{Panoramica}

Questo caso di studio fornisce una breve panoramica, per utenti principianti e di livello intermedio, tratta dal Report sulla tecnologia DPC sull'archiviazione Web. Sono inclusi tre "mini casi di studio" che illustrano i diversi contesti operativi, driver e soluzioni che possono essere implementate. II report stesso fornisce una discussione dettagliata su diverse questioni e pratiche con ulteriori approfondimenti e consigli (Pennock, 2013). Consigliato ai lettori che necessitano di un briefing di livello più avanzato sull'argomento e sulle attività pratiche.

\section{Introduzione}

II World Wide Web è una risorsa di informazioni unica su vasta scala, utilizzata a livello globale. Gran parte dei suoi contenuti avrà probabilmente valore non solo per la generazione attuale ma anche per quelle future. Tuttavia il grande lascito del web è a rischio, in parte minacciato dalla stessa velocità con cui è diventato un successo. II contenuto viene perso a un ritmo allarmante, mettendo a rischio non solo la nostra memoria culturale digitale ma anche la responsabilità organizzativa. Pertanto, un certo numero di istituti di beni culturali e accademici, organizzazioni senza scopo di lucro e imprese private hanno esplorato i problemi coinvolti e guidato o contribuito allo sviluppo di soluzioni tecniche per l'archiviazione web.

\section{Servizi e soluzioni}

Le esigenze aziendali e le risorse disponibili sono considerazioni fondamentali nella scelta degli strumenti e/o dei servizi di archiviazione web appropriati. Si devono tenere in conto anche altri aspetti: le organizzazioni che prendono in considerazione l'archiviazione web per soddisfare i requisiti normativi devono, ad esempio, valutare questioni congiunte come l'autenticità e l'integrità, la conservazione e la garanzia della qualità. Tutte le organizzazioni dovranno prendere in considerazione la questione della selezione (ovvero quali siti Web archiviare), un compito apparentemente semplice, che viene complicato dalle difficili interrelazioni condivise dalla maggior parte dei siti Web che non rendono facile stabilire dei limiti. Altre questioni includono la gestione dei malware, la riduzione al minimo della duplicazione delle risorse, la 
coerenza temporale dei siti e la conservazione a lungo termine o la sostenibilità delle risorse. La collaborazione internazionale si sta rivelando un punto di svolta nello sviluppo di soluzioni scalabili per supportare la conservazione a lungo termine e garantire che le collezioni rimangano affidabili per le generazioni future.

Il processo di archiviazione web non è un'azione una tantum. Una suite di applicazioni viene in genere distribuita per supportare le diverse fasi del processo, sebbene possano essere integrate in un singolo flusso di lavoro end-to-end. Gran parte del software è disponibile come open source, consentendo alle istituzioni di accedere gratuitamente al codice sorgente per l'uso e/o la modifica senza alcun costo.

\section{Sistemi integrati per l'archiviazione Web}

Un numero limitato di sistemi integrati è disponibile per coloro i quali dispongono di personale tecnico sufficiente per installare, mantenere e amministrare un sistema internamente. In genere, offrono funzionalità integrate di archiviazione Web per gran parte del ciclo di vita, dalla selezione e gestione delle autorizzazioni alla ricerca per l'indicizzazione, il controllo qualità e l'accesso. Di seguito ne vengono illustrati tre.

\section{PANDAS}

PANDAS (PANDORA Digital Archiving System) è stato uno dei primi sistemi integrati di archiviazione web disponibili. Implementato per la prima volta dalla National Library of Australia (NLA) nel 2001, PANDAS è un'applicazione Web scritta in Java e Perl che fornisce un'interfaccia intuitiva per gestire il flusso di lavoro di archiviazione web. Supporta la selezione, le autorizzazioni, la programmazione, la raccolta, il controllo qualità, l'archiviazione e l'accesso. PANDAS non è un software open source, sebbene sia stato utilizzato da altre istituzioni (in particolare il consorzio di archiviazione Web del Regno Unito dal 2004 al 2008). Viene utilizzato dall'NLA per l'archiviazione Web selettiva, mentre Internet Archive supporta le loro istantanee annuali del dominio australiano.

Web Curator Tools (WCT)

II Web Curator Tools è uno strumento di flusso di lavoro open source per la gestione del processo selettivo di archiviazione Web, sviluppato in collaborazione dalla National Library of New Zealand e dalla British Library con Oakleigh Consulting. Supporta la selezione, le autorizzazioni, la descrizione, la raccolta e la garanzia della qualità, con un'interfaccia di accesso separata. WCT è scritto in Java all'interno di un'architettura flessibile ed è disponibile pubblicamente per il download da SourceForge con una licenza pubblica Apache. II sito Web WCT è I'hub per la comunità di sviluppatori e ci sono mailing list attive sia per utenti che per sviluppatori. La natura altamente modulare del sistema ne minimizza le dipendenze.

\section{NetarchiveSuite}

NetarchiveSuite è un'applicazione di archiviazione web scritta in Java per la gestione dell'archiviazione Web selettiva e di ampi settori, originariamente sviluppata nel 2004 dalle due biblioteche che si occupano del deposito legale in Danimarca (Det Kongelige Bibliotek e Statsbiblioteket). È diventato open source nel 2007 e ha ricevuto ulteriori input di sviluppo dalla Bibliothèque nationale de France e dalla Österreichische Nationalbibliothek dal 2008. E 
liberamente disponibile sotto la GNU Lesser General Public License (LGPL). La natura altamente modulare del sistema consente soluzioni di implementazione flessibili.

\title{
Servizi di terzi e commerciali
}

I servizi di archiviazione web commerciale di parti terze sono sempre più utilizzati dalle organizzazioni che preferiscono non creare e mantenere la propria infrastruttura tecnica di archiviazione web. Le ragioni dietro questa scelta possono ampiamente variare. Spesso non si tratta semplicemente della portata dell'operazione o della complessità percepita, ma della necessità e del focus dell'azienda. Molte organizzazioni non desiderano investire in competenze o capitali che non siano fondamentali per la propria attività. Altri possono utilizzare tale servizio per evitare investimenti di capitale. Inoltre, le organizzazioni trasferiscono sempre più le proprie operazioni informatiche nel cloud o utilizzano un fornitore SAAS (Software as a Service). L'archiviazione Web non fa eccezione. Dal punto di vista legale e della conformità, i servizi di parti terze sono talvolta preferiti in quanto possono fornire non solo la tecnologia ma anche le competenze e il supporto necessari per soddisfare le esigenze aziendali. Questa sezione introduce alcuni dei servizi di parti terze attualmente disponibili, ma non è un elenco esaustivo, e la selezione qui di seguito riportata dovrebbe essere considerata come una raccomandazione.

\begin{abstract}
Archive-It
Archive-It è un servizio di archiviazione web in abbonamento fornito da Internet Archive. I clienti utilizzano il servizio per creare raccolte specifiche, ad esempio le Olimpiadi di Londra 2012, i siti Web governativi, i diritti umani e la bibliografia consigliata dei corsi universitari. Un'interfaccia utente dedicata viene fornita ai clienti per selezionare e gestire i seed, impostare lo scopo e la frequenza di crawling, monitorare il suo avanzamento ed eseguire controlli di qualità, aggiungere metadati e creare delle landing page per le loro raccolte. Le raccolte sono rese pubbliche per impostazione predefinita tramite il sito Web Archive-It, con raccolte private che richiedono un accordo speciale. L'interfaccia di accesso supporta la ricerca di URL e di testo completo. Oltre 200 partner utilizzano il servizio, principalmente dai settori accademici o di patrimonio culturale. II costo del servizio dipende dai requisiti dell'istituto.
\end{abstract}

\section{Archivethe.Net}

Archivethe.Net è un servizio di archiviazione web fornito dall'Internet Memory Foundation (IMF). Consente ai clienti di gestire l'intero flusso di lavoro tramite un'interfaccia Web su tre moduli principali: amministrazione (gestione utenti), raccolta (gestione seed e crawling) e report (report e metriche a diversi livelli). La piattaforma è disponibile in inglese e francese. Oltre alla ricerca fulltext e alla raccolta di contenuti multimediali, supporta anche un servizio di reindirizzamento automatico per i siti live. Sono in fase di sviluppo strumenti di Q\&A automatizzati, sebbene I'FMI possa anche fornire servizi manuali di garanzia della qualità, nonché la gestione diretta della raccolta per le istituzioni che non desiderano utilizzare lo strumento online. I costi dipendono dai requisiti dell'istituto. Le raccolte possono essere rese private o rimanere apertamente accessibili, nel qual caso possono essere contrassegnate e apparire nella raccolta del FMI. In questi casi il costo per l'hosting viene assorbito dal FMI.

\section{The University of California's Curation Centre (UC3)}

Come parte della California Digital Library, il Centro fornisce un servizio di archiviazione Web completamente ospitato per raccolte selettive di archivi Web. I dipartimenti e le organizzazioni dell'Università della California pagano solo per l'archiviazione. I costi per altri gruppi e consorzi 
comprendono una commissione di servizio annuale più i costi di conservazione. Le raccolte possono essere rese pubbliche o private. Circa 20 organizzazioni partner hanno reso disponibili raccolte fino ad oggi. Viene fornita la ricerca full text e la presentazione delle collezioni può essere contrassegnata su richiesta delle istituzioni.

\section{Società private}

Le società private offrono servizi di archiviazione web appositamente studiati per le esigenze aziendali. Gli archivi Hanzo, ad esempio, forniscono un servizio di archiviazione di siti Web commerciali per soddisfare le esigenze commerciali relative alla conformità normativa, all'ediscovery e alla gestione documentale. Gli archivi di Hanzo sottolineano la loro capacità di raccogliere siti e contenuti multimediali che potrebbero essere difficili da raccogliere per un crawler standard, inclusi contenuti dinamici da Sharepoint e wiki da internet privati, insieme a canali di social media pubblici e privati. (Maggiori dettagli sulle possibilità offerte dal servizio Archivi Hanzo sono disponibili nel caso di studio Coca-Cola) Allo stesso modo, Reed Archives fornisce un servizio di archiviazione web commerciale per la conformità alle normative organizzative, la protezione del contenzioso, l'eDiscovery e la gestione dei registry. Ciò include un set di strumenti "archiviare su richiesta" da utilizzare durante la navigazione sul Web. In ogni caso, il costo del servizio è adattato alle precise esigenze del cliente. Sono inoltre disponibili altre società e servizi e si esortano i lettori a cercare online ulteriori opzioni qualora tale servizio sia di interesse.

\section{Caso di studio 1: The UK Web Archive}

II UK Web Archive (UKWA) è stato istituito nel 2004 dal Web Archiving Consortium del Regno Unito. In origine era una partnership a sei, guidata dalla British Library in collaborazione con Wellcome Library, JISC, National Library of Wales, National Library of Scotland e The National Archives (Regno Unito).

I partner UKWA selezionano e citano i siti Web utilizzando le funzionalità del sistema di archiviazione Web ospitato nell'infrastruttura del Web Archive del Regno Unito gestita dalla British Library. La British Library lavora a stretto contatto con un certo numero di altre istituzioni e individui per selezionare e citare siti Web di interesse. I siti Web archiviati in modo selettivo vengono rivisitati a intervalli regolari in modo da rilevare i cambiamenti nel tempo.

L'infrastruttura tecnica alla base del UK Web Archive è gestita dalla British Library. L'archivio è stato originariamente creato con il software PANDAS fornito dalla National Library of Australia, ospitato da un'agenzia esterna, ma nel 2008 l'archivio è stato spostato internamente e migrato nel sistema Web Curator Tools (WCT).

Una versione personalizzata dell'interfaccia Wayback sviluppata da Internet Archive viene utilizzata come front-end WCT e fornisce un accesso ricercabile a tutti i siti Web archiviati pubblicamente disponibili. La ricerca full-text è abilitata e si aggiunge alle ricerche standard per titolo e URL e a uno schema di classificazione dei soggetti. II team di archiviazione web della biblioteca ha recentemente rilasciato una serie di strumenti di visualizzazione per aiutare i ricercatori a comprendere e trovare contenuti nella raccolta. 
Sono state istituite raccolte speciali su un'ampia gamma di argomenti. Molti sono a carattere tematico, ad esempio la salute mentale e le raccolte della Chiesa libera. Altri documentano la risposta online a un evento storico recente di notevole interesse, come le elezioni generali del Regno Unito, il Diamond Jubilee della Regina Elisabetta II e le Olimpiadi di Londra 2012.

Molti altri siti singoli, non associati a una determinata raccolta speciale, sono stati archiviati su raccomandazione di esperti in materia o membri del pubblico. Questi spesso non sono più disponibili sul live web, ad esempio il sito web del parlamentare britannico Robin Cook o del progetto di arte pubblica One \& Other di Antony Gormley, acquisito da Sky Arts.

\section{Caso di studio 2: Internet Memory Foundation}

La Internet Memory Foundation (FMI) è stata fondata nel 2004 come organizzazione no profit per supportare le iniziative di archiviazione web e sviluppare una forma di sostegno per la conservazione del web in Europa. Originariamente conosciuta come European Archive Foundation, ha cambiato il suo nome nel 2010. L'IMF offre ai clienti una soluzione di archiviazione web completa e esternalizzata per gestire il flusso di lavoro di archiviazione web senza che debbano affrontare i problemi del flusso di lavoro operativo.

L'IMF collabora strettamente con la Internet Memory Research (IMR) per gestire una parte dei suoi flussi di lavoro tecnici per l'archiviazione web. L'IMR è stato istituito nel 2011 come spin-off dall'IMF. Sia I'IMF che I'IMR sono coinvolti in progetti di ricerca a supporto della crescita e dell'uso degli archivi web.

IMR fornisce un servizio di archiviazione web personalizzabile, Archivethe.Net (AtN). AtN è una piattaforma di archiviazione Web condivisa con un'interfaccia basata sul Web che aiuta le istituzioni a iniziare facilmente e rapidamente a raccogliere siti Web che includono contenuti dinamici e rich media. Può essere adattata alle esigenze dei clienti e le istituzioni mantengono il pieno controllo della loro politica di raccolta (capacità di selezionare i siti, di specificare la profondità, la frequenza di raccolta, ecc.). Servizi di controllo di qualità possono essere forniti su richiesta. La maggior parte viene eseguita manualmente al fine di soddisfare elevati livelli di requisiti di qualità istituzionale $\mathrm{e} \mathrm{IM}$ dispone di un team di controllo qualità dedicato composto da valutatori del controllo qualità. La messaggistica istantanea ha sviluppato una metodologia per il confronto visivo basata su strumenti utilizzati per la ricerca, per l'indicizzazione e l'accesso ai dati, sebbene stiano anche lavorando per migliorare strumenti e metodi per offrire una qualità di ricerca per l'indicizzazione iniziale di livello più alto.

Le istituzioni partner, con raccolte apertamente accessibili per le quali I'IM fornisce un servizio di archiviazione web, includono gli Archivi nazionali del Regno Unito e il Parlamento del Regno Unito. 
L'accesso alle raccolte disponibili al pubblico è fornito tramite il sito Web IM. IM offre una funzione di ricerca full-text per la maggior parte delle sue raccolte online, oltre alla ricerca basata su URL. I risultati della ricerca full-text possono essere integrati in un sito Web di terzi e alle collezioni può essere associato un marchio dai proprietari secondo le loro necessità.

Seguendo l'architettura del Web Continuity Service di The National Archives (The National Archives, 2010), IM ha implementato un "servizio di reindirizzamento automatico" per integrare gli archivi Web con l'esperienza utente live web. Durante la navigazione sul Web, gli utenti vengono reindirizzati automaticamente all'archivio Web se la risorsa richiesta non è più disponibile online. All'interno dell'archivio Web, l'utente viene indirizzato all'istanza più recentemente acquisita relativa alla risorsa richiesta. Una volta effettuato l'accesso alla risorsa, qualsiasi collegamento sulla pagina riporterà l'utente alla versione live del sito. Si ritiene che questo servizio possa aumentare la durata di un link, migliorare l'esperienza degli utenti, la visibilità, il ranking online e ridurre le frequenze di rimbalzo.

Le raccolte di archiviazione Web sono disponibili per la navigazione pubblica dal sito Web di IM, una combinazione delle proprie raccolte selettive e di dominio, e quelle delle istituzioni partner.

\section{Caso di studio 3: I'archivio web della Coca-Cola}

L'archivio web della Coca-Cola è stato istituito per acquisire e preservare i siti Web e i social media aziendali della Coca-Cola. Fa parte dell'Archivio Coca-Cola, che contiene milioni di manufatti sia fisici che digitali, da documenti e fotografie a pubblicità, bottiglie e articoli promozionali. La presenza online di Coca-Cola è vasta, compresi non solo i diversi siti web nazionali di Coca-Cola ma anche, ad esempio, la pagina Facebook e lo stream di Twitter di Coca-Cola e altri marchi di proprietà di Coca-Cola (500 in tutto). II primo sito della Coca-Cola è stato pubblicato nel 1995.

Dal 2009, Coca-Cola collabora con gli archivi di Hanzo e ora utilizza il loro servizio di archiviazione web commerciale. Oltre ai vantaggi storici dell'archivio Web, il servizio fornisce anche supporto per controversie legali in cui parte o tutto il sito Web può essere richiesto come prova in tribunale e conformità alla normativa per le applicazioni di gestione documentale.

L'archivio web Coca-Cola è uno speciale archivio web a tema che contiene tutti i siti aziendali Coca-Cola e altri siti appositamente selezionati associati alla Coca-Cola. È inteso per essere il più completo possibile, con integrità/funzionalità dei siti acquisiti di primaria importanza. Ciò include social media e video, sia live streaming che incorporati (incluso Flash). I manufatti sono conservati nella loro forma originale ove possibile, un principio fondamentale per tutti gli oggetti nell'archivio Coca-Cola.

L'acquisizione degli archivi di Hanzo si svolge trimestralmente ed è integrata da acquisizione di dati occasionali riferiti ad eventi particolari, ad esempio il $125^{\circ}$ anniversario di Coca-Cola, 
celebrato nel 2011. La soluzione di archiviazione web di Hanzo è un'applicazione personalizzata. II contenuto Web viene raccolto nel suo formato nativo dal web crawler di Hanzo Archives, che viene distribuito adattandosi alla portata richiesta per l'attività in corso.

La garanzia della qualità viene effettuata con un controllo sistematico a doppio salto dei contenuti ottenuti dall'acquisizione che impone l'uso delle opzioni di navigazione di livello superiore e si concentra sulla forma tecnica del sito.

L'archivio è attualmente accessibile solo ai dipendenti della Coca-Cola, su un numero limitato di macchine. L'accesso remoto è fornito da Hanzo utilizzando la propria interfaccia di accesso. L'accesso basato su proxy garantisce che tutto il contenuto sia offerto direttamente dall'archivio e che non si verifichino "perdite dal sito". L'archivio potrebbe essere reso pubblicamente accessibile in futuro all'interno di The World of Coca-Cola, ad Altanta, Georgia, USA.

La raccolta di archivi web Coca-Cola contiene oltre sei milioni di pagine Web e oltre 2 TB di dati. Prima della loro collaborazione con Hanzo, i primi tentativi di archiviazione hanno comportato acquisizioni incomplete, quindi i primi siti non sono così completi come vorrebbe la società. La collezione contiene anche informazioni su molti eventi nazionali e internazionali di cui Cola-Cola è stata sponsor, tra cui le Olimpiadi di Londra 2012 e il Diamond Jubilee della Regina Elisabetta II.

\section{Conclusioni}

La tecnologia di archiviazione Web è maturata notevolmente negli ultimi dieci anni, così come una maggiore consapevolezza delle problematiche che comporta. Di conseguenza disponiamo di una vasta gamma di strumenti e di servizi che ci consentono di archiviare e preservare aspetti della nostra memoria culturale online e di rispettare i requisiti normativi per l'acquisizione e la conservazione dei record online. II lavoro è ancora in corso, finché Internet continua a evolversi, la tecnologia di archiviazione web deve evolversi per stare al passo.

Oltre agli sviluppi tecnici, le conoscenze e l'esperienza acquisite attraverso l'implementazione pratica e l'uso degli strumenti di archiviazione Web hanno portato a una comprensione migliore delle buone pratiche nel campo dell'archiviazione Web, strategie operative per incorporare l'archiviazione Web in un contesto organizzativo, esigenze e vantaggi aziendali, casi d'uso e opzioni di risorse. Le organizzazioni che desiderano intraprendere un'iniziativa di archiviazione web devono essere molto chiare sulle proprie esigenze aziendali prima di farlo. Le esigenze aziendali dovrebbero essere il driver fondamentale dietro qualsiasi iniziativa di archiviazione web e influenzeranno in modo significativo i dettagli di una conseguente strategia di archiviazione web e le politiche di selezione. Il fatto che siano emersi servizi e tecnologie commerciali è un segno della maturità dell'archiviazione Web come necessità aziendale e disciplina. 


\section{Risorse}

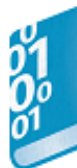

Pennock, M., 2013. Web-Archiving, DPC Technology Watch Report 13-01 March 2013 http://dx.doi.org/10.7207/twr13-01

Questo report è destinato a coloro i quali sono interessati o responsabili della creazione di un archivio web. Illustra le questioni chiave affrontate dalle organizzazioni impegnate in iniziative di archiviazione web; se si stanno affidando a un fornitore di servizi di parti terze o se stanno gestendo il processo internamente, e fornisce una panoramica dettagliata delle principali applicazioni e software attualmente disponibili.

ISO, 2012, ISO 28500:2009 Information and Documentation - the WARC file format http://www.iso.org/iso/catalogue detail.htm?csnumber $=44717$

II formato WARC (Web ARChive) è un formato contenitore per siti Web archiviati, noto anche come ISO 28500:2009. È una revisione del formato di file ARC di Internet Archive utilizzato per archiviare Web crawl raccolte dal World Wide Web.

ISO, 2013 ISO/TR 14873:2013 Information and Documentation - Statistics and quality issues for web archiving

http://www.iso.org/iso/catalogue detail.htm?csnumber=55211

Questo rapporto tecnico definisce statistiche, termini e criteri di qualità per l'archiviazione Web. Considera le esigenze e le pratiche di una vasta gamma di organizzazioni come biblioteche, archivi, musei, centri di ricerca e fondazioni del patrimonio culturale.

Meyer E 2010 (a), Researcher Engagement with Web Archives: State of the Art Report, JISC

http://ie-repository.jisc.ac.uk/544/

Questo report sintetizza lo stato dell'arte dell'archiviazione web in relazione ai ricercatori e alle esigenze di ricerca concentrandosi principalmente su singoli ricercatori e istituzioni.

Perma: Scoping and Addressing the Problem of Link and Reference Rot in Legal Citations

Zittrain, Jonathan and Albert, Kendra and Lessig, Lawrence, Perma: Scoping and Addressing the Problem of Link and Reference Rot in Legal Citations (October 1, 2013). Harvard Public Law Working Paper No. 13-42. Disponibile presso SSRN: http://ssrn.com/abstract=2329161 o http://dx.doi.org/10.2139/ssrn.2329161

http://papers.ssrn.com/sol3/papers.cfm?abstract id=2329161

http://dx.doi.org/10.2139/ssrn.2329161

Questo articolo redatto dal team del progetto Perma documenta il problema del mancato collegamento: oltre il $70 \%$ degli URL all'interno della Harvard Law Review e altre riviste e il 50\% degli URL trovati nei pareri della Corte Suprema degli Stati Uniti, non si collega alle informazioni 
originariamente citate. Propone una soluzione per autori ed editori e coinvolge le biblioteche che si occupano della conservazione distribuita a lungo termine dei contenuti dei link.

\section{Scholarly Context Not Found: One in Five Articles Suffers from Reference Rot http://dx.doi.org/10.1371/journal.pone.0115253}

Questo studio su larga scala ha esaminato circa 600.000 link estratti da oltre 3 milioni di articoli accademici pubblicati tra il 1997 e il 2012. Questi erano link a cosiddette risorse web-at-large, ovvero non link ad altri articoli accademici. Ha individuato un articolo STM su cinque caratterizzato da mancato collegamento, il che significa che è impossibile rivisitare il contesto web che li circonda qualche tempo dopo la loro pubblicazione. Quando si considerano solo gli articoli STM che contengono riferimenti a risorse Web, questa frazione aumenta a sette su dieci.

The National Archives, 2010.Government Web Archive: Redirection Technical Guidance for Government Departments, version 4.2, The National Archives (UK)

http://www.nationalarchives.gov.uk/documents/information-management/redirection-technicalguidance-for-departments-v4.2-web-version.pdf

Questa guida descrive un servizio innovativo che fornisce funzionalità di riscrittura e reindirizzamento URL per le pagine Web del governo del Regno Unito impostando il reindirizzamento all'archivio Web del governo del Regno Unito nel caso in cui un URL richiesto non esiste più su un sito Web dipartimentale.

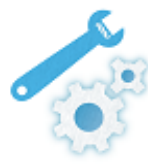

\section{MEMENTO and the Time Travel Service}

http://www.mementoweb.org/

Memento è uno strumento che consente agli utenti di vedere la versione di una risorsa Web che esisteva ad un certo punto nel passato. Ora è usato in diversi archivi web. Il servizio Time Travel basato su Memento controlla una serie di server tra cui molti archivi web e cerca di trovare una pagina web per come si presentava in un periodo a scelta.

\section{Archive-lt}

http://www.archive-it.org/

Archivethe.Net

http://www.archivethe.net/en/

Hanzo Archives

http://www.hanzoarchives.com/

Internet Memory Foundation \& Internet Memory Research

http://www.internetmemory.org/en/ 
Wayback

http://www.sourceforge.net/projects/archive-access/files/wayback/

Netarchive Suite

https://sbforge.org/display/NAS/NetarchiveSuite

PANDAS

http://pandora.nla.gov.au/pandas.html

Reed Archives

http://www.reedarchives.com/

UC3 Web Archiving Service

http://www.collib.org/services/uc3/was.html

Web Curator Tools

http://webcurator.sourceforge.net/

WWW

\section{International Internet Preservation Consortium}

http://www.netpreserve.org

IIPC è un'organizzazione associativa dedicata al miglioramento degli strumenti, degli standard e delle migliori pratiche di archiviazione web promuovendo al contempo la collaborazione internazionale e l'ampio accesso e utilizzo degli archivi web per la ricerca e il patrimonio culturale. Ci sono molte risorse preziose sul sito Web tra cui eccellenti brevi video come nell'esempio seguente.

Why Archive the Web?

https://www.youtube.com/watch?v=pU32riTaMFE

Un breve video pubblicato il 18 ottobre 2012 che presenta le sfide relative all'archiviazione Web e l'IIPC. (2 minuti e 53 secondi).

What is a Web Archive?

https://youtu.be/ubDHY-ynWi0

Questo breve video spiega il "Web Archiving" e perché è importante che le biblioteche responsabili del deposito legale nel Regno Unito lo supportino. È stato prodotto nell'ambito del progetto Arts and Humanities Research Council finanziato dal progetto "Big UK Domain Data for the Arts and Humanities". (2 minuti e 31 secondi) 
What do the UK Web Archive collect?

https://youtu.be/1QLMPIRwJEo

Questo video spiega agli utenti cosa possono aspettarsi di trovare e dove potrebbero cercare per accedere alle tre raccolte che detengono il Web Archive del Regno Unito. È stato prodotto nell'ambito del progetto Arts and Humanities Research Council finanziato dal progetto "Big UK Domain Data for the Arts and Humanities". (2 minuti 55 secondi)

\section{Ulteriori casi di studio}

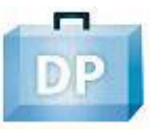

Casi di studio sui contenuti del sito Web NDSA

La National Digital Stewardship Alliance (NDSA) degli Stati Uniti esamina il valore, le opportunità e gli ostacoli per la conservazione selettiva dei seguenti tipi di contenuti Web specifici:

Science, Medicine, Mathematics, and Technology forums

http://www.digitalpreservation.gov/ndsa/working groups/documents/ScienceForums CaseStu

dy public v2.pdf

Dicembre 2013 (3 pagine).

Science, Medicine, Mathematics, and Technology blogs

http://www.digitalpreservation.gov/ndsa/working groups/documents/ScienceBlogs CaseStudy public v2.pdf

Dicembre 2013 (3 pagine).

Born-Digital Community and Hyperlocal News

http://www.digitalpreservation.gov/ndsa/working groups/documents/NDSA CaseStudy Com

munityNews.pdf

Febbraio 2013 (3 pagine).

Citizen Journalism

http://www.digitalpreservation.gov/ndsa/working groups/documents/NDSA CaseStudy Citize nJournalism.pdf

Febbraio 2013 (3 pagine).

On the Development of the University of Michigan Web Archives: Archival Principles and Strategies

http://files.archivists.org/pubs/CampusCaseStudies/Case13Final.pdf

Michael Shallcross, Bentley Historical Library, University of Michigan descrive in dettaglio le strategie e le procedure seguite dal programma Archives and Records (UARP) dell'Università per sviluppare la sua raccolta di siti Web archiviati e come ha avviato un progetto di conservazione di siti Web su larga scala nell'ambito di uno sforzo più ampio: acquisire e conservare in modo proattivo documenti elettronici selezionati dell'Università. 2011 (29 pagine). 


\section{Riferimenti}

Pennock, M., 2013. Web-Archiving, DPC Technology Watch Report 13-01 March 2013. Disponibile al seguente link: http://dx.doi.org/10.7207/twr13-01

The National Archives, 2010. Government Web Archive: Redirection Technical Guidance for Government Departments, version 4.2, The National Archives (UK). Disponibile al seguente link: http://www.nationalarchives.gov.uk/documents/information-management/redirection-technicalguidance-for-departments-v4.2-web-version.pdf 


\section{Glossario}

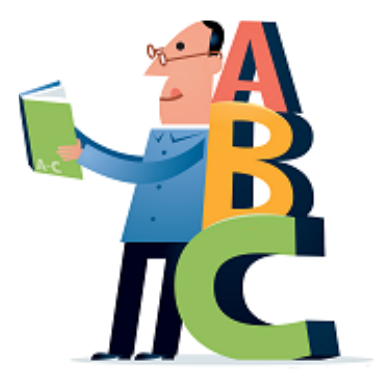

Illustrazione di Jørgen Stamp digitalbevaring.dk CC BY 2.5 Denmark

\section{Introduzione}

Gli Acronimi e le Iniziali sono propri di ogni dominio specialistico. All'interno di una disciplina emergente, come quella della conservazione digitale, un'altra difficoltà fondamentale è legata alla mancanza di una tassonomia precisa e definitiva dei termini. Diverse comunità utilizzano gli stessi termini in modi diversi, il che può rendere problematica qualsiasi tipologia di comunicazione effettiva.

Il seguente insieme di definizioni e acronimi riprende quelli utilizzati all'interno di tutto il Manuale e dai DPC Technology Watch Reports e Website, supportandone l'uso come pratico strumento. La traduzione è stata fatta in maniera letterale, ma alcuni termini hanno una diversa accezione nel conteso normativo italiano ed europeo per cui si rinvia, comunque, all'allegato 1 delle Linee Guida sulla Formazione Gestione e Conservazione dei Documenti Informatici25.

A

Accesso Come definito nel Manuale, per accesso si intende un utilizzo continuo, costante della risorsa digitale, mantenendo tutte le qualità di autenticità, accuratezza e funzionalità ritenute essenziali per gli obiettivi per cui è stata creata/acquisita la risorsa digitale.

Accesso continuo si riferisce al diritto di un abbonato a una pubblicazione elettronica e dei suoi utenti di avere un accesso permanente e costante alle risorse elettroniche che sono state già prese in prestito o acquisite dall'abbonato medesimo presso un editore. È un termine utilizzato nell'industria informatica, insieme ai suoi sinonimi - accesso perpetuo e accesso postcancellazione - per descrivere la capacità di mantenere l'accesso alle risorse elettroniche attraverso utenti abbonati/licenziatari dopo che un accordo contrattuale di autorizzazione con

${ }^{25} \mathrm{https://trasparenza.agid.gov.it/archivio19} \mathrm{regolamenti} 0 \quad 5385 . \mathrm{html}$ 
l'editore/licenziatario per quelle risorse è terminato, qualunque sia la ragione della cessazione. Si tratta anche, ove opportuno, di accordi per la conservazione digitale necessari a garantire alcuni elementi per un accesso continuo.

Accesso permanente si veda Accesso continuo.

Accesso post-cancellazione si veda Accesso continuo.

ADS Archaeology Data Service. Un servizio britannico attivo nella conservazione digitale. http://ads.ahds.ac.uk

Aggiornamento Copiare il contenuto informativo da un supporto di archiviazione sullo stesso supporto di archiviazione.

AIP Archival Information Package. Un pacchetto informativo che è costituito dal Contenuto Informativo e dalla Description Information (PDI) associata.

AMIA Association of Moving Image Archives, un'organizzazione attiva nel campo dell'archiviazione di immagini in movimento. http://www.amianet.org

ARC Formato contenitore per i siti web sviluppati dall'Internet Archive, sostituito da WARC.

Archiviazione digitale Questo termine viene usato in modo molto diverso all'interno dei diversi settori. Le comunità delle biblioteche e degli archivi lo usano spesso intercambiabilmente con conservazione digitale. Gli informatici tendono a usarlo per indicare il processo di backup e mantenimento costante in contrasto con strategie di conservazione a lungo termine. In questo Manuale si usa quest'ultima accezione più completa, definita come conservazione digitale.

Archivio Dim garantisce una conservazione di bit per il contenuto, un piano di conservazione digitale e azioni per un accesso perpetuo a lungo termine, e anche un accesso attuale limitato (probabilmente limitato a utenti in loco o abbonati precedenti alla post-cancellazione, ecc.).

ASCII American Standard Code for Information Interchange, standard per i testi elettronici. https://en.wikipedia.org/wiki/ASCII

Assicurazione delle informazioni Un aspetto della sicurezza digitale che mira specificamente a garantire che la qualità delle informazioni venga tutelata in modo comprovabile, che non sia stata alterata o che ci siano stati accessi inappropriati.

Autenticazione Un meccanismo che cerca di stabilire l'autenticità delle risorse digitali in un punto preciso nel tempo. Ad esempio, le firme digitali.

Autenticità La risorsa digitale è ciò che afferma di essere. Nel caso dei documenti elettronici, l'autenticità si riferisce all'affidabilità del documento elettronico in quanto documento. Nel caso dei 'nativi digitali' e delle risorse digitalizzate, essa si riferisce al fatto che indipendentemente da ciò che vienemenzionato, il contenuto sia lo stesso di quando è stato originariamente creato a meno che i metadati di accompagnamento non indichino qualche cambiamento. Essere sicuri dell'autenticità delle risorse digitali nel tempo è molto importante constatata la facilità con cui possono verificarsi delle modifiche. 
Blocco dei diritti di scrittura Strumenti che impediscono ai sistemi informatici di valutazione di scrivere o alterare una collezione o dischi rigidi o altri oggetti su supporto digitale. I sistemi hardware di blocco dei diritti di scrittura sono generalmente considerati più affidabili rispetto ai sistemi software di blocco dei diritti di scrittura.

Bit Un bit è l'unità di base delle informazioni in informatica. Può avere solo uno o due valori rappresentati comunemente sia da 0 che da 1 . I due valori possono essere interpretati come qualsiasi attributo binario (si/no, on/off, ecc).

BWF II formato Broadcast WAV format, lo standard European Broadcasting Union per un file WAV con metadati extra. http://www.digitalpreservation.gov/formats/fdd/fdd000003.shtml

Byte (B) Un'unità di informazione digitale che nella maggior parte dei casi consiste in otto bit. Storicamente, con byte si indica un numero di bit usato per decodificare un singolo carattere di testo nel computer, e per questa ragione esso è la più piccola unità di memoria indirizzabile in molti sistemi informatici.

\section{C}

CCDSDS Consultative Committee for Space Data Systems, I'organismo responsabile del Modello di riferimento OAIS. http://public.ccsds.org/default.aspx

Catena di custodia Un concetto chiave in ambito legale con cui la custodia e la provenienza di un hardware digitale, dei media e dei file, vengono tutelate attraverso, per esempio, la nomina degli evidence custodians. L'obiettivo della Digital Evidence Bag (DEB) è di mantenere in forma digitale, insieme agli oggetti digitali probatori, i metadati di provenienza che possono essere aggiornati ove richiesto: un concetto familiare ai professionisti del settore della conservazione digitale.

Checksum Una firma numerica unica proveniente da un file. Usata per la comparazione delle copie.

CLIR Council on Library and Information Resources. Un'organizzazione americana attiva nella conservazione digitale. http://www.clir.org

CNI Coalition for Networked Information. Un'organizzazione americana attiva nella conservazione digitale. http://www.cni.org

Compressione lossless (compressione dati senza perdita) Un meccanismo di riduzione della dimensione dei file che mantiene inalterati gli elementi informativi dei dati originali.

Compressione lossy (compressione dati con perdita) Un meccanismo di riduzione della dimensione dei file che potrebbe comportare la perdita di elementi informativi.

Comunità designata Un gruppo determinato di potenziali utenti che dovrebbe essere in grado di comprendere un particolare insieme di informazioni da un archivio. Questi utenti potrebbero 
essere comunità multiple, designate da un archivio, e possono cambiare nel tempo (termine OAIS).

Conservazione dei Bit Termine usato per denotare un livello molto generale per la conservazione della risorsa digitale nel momento in cui questa viene versata (letteralmente la conservazione dei bit che forma una risorsa digitale). Potrebbe comprendere il mantenimento in loco o esterno delle copie di backup, il controllo dei virus, il controllo della fixity, un aggiornamento periodico verso nuovi supporti di archiviazione. La conservazione dei bit non è da intendersi come conservazione digitale, ma comunque fornisce una componente importante per un insieme più complesso di pratiche e processi di conservazione digitale che garantiscono la sussistenza del contenuto digitale e la sua fruibilità, presentazione, contesto e interpretazione nel tempo.

Conservazione digitale Si riferisce a una serie di attività organizzate necessarie per assicurare un accesso costante alle risorse digitali finché sarà necessario. La definizione di conservazione digitale è molto generale ai fini di questo studio e si riferisce a tutte le azioni necessarie per mantenere l'accesso alle risorse digitali al di là dei limiti dovuti ai guasti dei supporti o ai cambiamenti tecnologici e organizzativi. Per risorse si possono intendere i record creati durante le attività quotidiane di un'organizzazione; risorse 'native-digitali' create per scopi specifici (ad es., risorse didattiche); o i prodotti dei progetti di digitalizzazione. In questo Manuale si esclude l'utilizzo potenziale della tecnologia digitale per conservare gli artefatti originali attraverso la digitalizzazione. Si veda la definizione di Digitalizzazione più in basso.

- Conservazione a breve termine - Accesso alle risorse digitali per un periodo di tempo stabilito nel momento in cui si prevede il suo utilizzo che non va oltre il futuro prossimo e/o fin quando non diventano inaccessibili a causa dei cambiamenti avvenuti nella tecnologia.

- Conservazione a medio termine - Accesso continuo alle risorse digitali al di là dei cambiamenti nella tecnologia per un periodo di tempo stabilito, ma non all'infinito.

- Conservazione a lungo termine - Accesso continuo alle risorse digitali, o per lo meno alle informazioni contenute in esse, all'infinito.

Controllo della fixity Un metodo che garantisce l'integrità di un file e verifica se è stato alterato o danneggiato. Durante il trasferimento un archivio può eseguire un controllo della fixity per assicurarsi che il file trasmesso non sia stato alterato nel percorso. All'interno di un archivio il controllo della fixity è una pratica che si usa per assicurare che i file digitali non siano stati alterati o danneggiati. Spesso viene realizzata calcolando i checksum come MD5, SHA1 o SHA256 per un file, e comparandoli con un valore archiviato. http://en.wikipedia.org/wiki/File_Fixity

COPTR Community Owned digital Preservation Tools Registry ospitato da The Open Preservation Foundation. http://coptr.digipres.org

Crawl L'attività di ricerca in rete automatica e sistematica per indicizzare o scaricare dei contenuti o altri dati dal web. II software utilizzato per fare crawl è spesso chiamato crawler. 
Dark Archive È un archivio a cui nessun utente può accedere, ma che potrebbe diventare accessibile in momenti futuri in conseguenza di determinati eventi ('eventi scatenanti'). L'accesso ai dati può essere limitato a un piccolo gruppo di utenti o essere completamente chiuso a tutti.

DCC Digital Curation Centre. Un'organizzazione UK attiva nella conservazione digitale. http://www.dcc.ac.uk

DDI Data Documentation Initiative. Uno standard ufficiale internazionale per la descrizione dei dati relativi alle scienze sociali, comportamentali ed economiche. http://www.icpsr.umich.edu/DDI

DigCurV Digital Curator Vocational Education Europe. Un progetto finanziato dalla Commissione Europea per la definizione di una struttura curriculare per la formazione professionale nella digital curation. http://www.digcurv.gla.ac.uk/

Digitalizzazione II processo relativo alla creazione di file digitali mediante scannerizzazione o, in alternativa, attraverso la conversione delle risorse analogiche. La copia digitale che ne risulta, o surrogato digitale, verrebbe poi classificata come risorsa digitale e sottoposta alle stesse sfide generali che emergono nel momento in cui se ne preserva l'accesso, così come per le risorse 'native-digitali'.

DIP Dissemination Information Package. Un pacchetto informativo, derivato da uno o più Archival Information Packages (AIPs), e inviato da parte degli Archivi agli utenti in risposta a una richiesta fatta a $\underline{\text { OAIS }}$ (termine OAIS).

DLF Digital Library Federation. Un'organizzazione americana attiva nella conservazione digitale. http://www.diglib.org

Documentazione Le informazioni fornite dal creatore e dal repository per poter stabilire la provenienza, la storia e il contesto delle risorse digiali al fine di permetterne l'utilizzo. Si veda anche Metadati.

\section{Documenti Digitali Vedi Documenti Elettronici.}

Documenti elettronici Record creati digitalmente durante le attività ordinarie di un'organizzazione che vi attribuisce uno status formale. Possono includere ad esempio i documenti di testo, email, database, o pagine web Intranet.

DOI Digital Object Identifier. Un'infrastruttura tecnica e organizzativa per la registrazione e l'uso di identificatori persistenti ampiamente utilizzati nelle pubblicazioni digitali e nei dati delle ricerche. II sistema DOI è stato creato dalla International DOI Foundation e poi adottato come International Standard ISO 26324 nel 2012.http://www.doi.org

DPC Digital Preservation Coalition. Un'organizzazione britannica e irlandese attiva nella conservazione digitale e responsabile del Manuale Digital Preservation Handbook. http://www.dpconline.org 
DPTP Digital Preservation Training Programme, un corso di formazione intensivo tenuto dallo University of London Computer Centre. https://dptp.london.ac.uk/

DRAMBORA Digital Repository Audit Methodology Based on Risk Assessment. Un insieme di strumenti di valutazione del rischio sviluppato dal Digital Curation Centre. http://www.dcc.ac.uk/resources/repository-audit-and-assessment/drambora

DROID Uno strumento di profilazione di file sviluppato e distribuito da TNA per l'identificazione dei formati dei file. Si basa su PRONOM. http://www.nationalarchives.gov.uk/informationmanagement/manage-information/policy-process/digital-continuity/file-profiling-tools-droid/

\section{$\mathbf{E}$}

Emulazione Un modo per superare l'obsolescenza dell'hardware e del software sviluppando delle tecniche atte ad emulare i sistemi obsoleti su future generazioni di computer.

Escrow Una diffusa pratica legale di deposito del contenuto o di un codice sorgente software con una parte terza. Escrow è presente nell'ambito di relazioni contrattuali, formalizzate in un accordo escrow, almeno tra tre parti: fornitore, utente, e la parte terza che fornisce il servizio escrow.

Evento scatenante Questa termine viene utilizzato quando si verificano delle specifiche condizioni riferite a una pubblicazione elettronica e al suo rilascio continuo per gli utenti. Se la pubblicazione non è più disponibile per gli utenti da parte di un editore o di una qualsiasi altra fonte, per una serie di motivazioni, allora si dice che si è verificato un evento scatenante. Questi eventi rendono possibile l'accesso per gli utenti attraverso un archivio in cui la pubblicazione elettronica potrebbe essere conservata digitalmente.

\section{$\mathbf{F}$}

FIAF International Federation of Film Archives, un'associazione leader mondiale degli archivi cinematografici. http://www.fiafnet.org

FIAT International Federation of Television Archives, un'associazione professionale per chi si occupa di conservazione e utilizzo di archivi di trasmissione. http://fiatifta.org

Formato dei file Un formato dei file è una modalità standard in cui l'informazione viene codificata per l'archiviazione in un archivio elettronico. Indica al computer come presentare, stampare, processare e salvare le informazioni. È gestito dal programma applicativo che ha creato il file e dal sistema operativo sotto il quale è stato creato e archiviato. Alcuni formati dei file sono progettati per dei tipi molto particolari di dati, altri possono funzionare come contenitori per altre tipologie. Uno specidico tipo di formato di formato viene spesso indicato dall'estensione del nome del file che contiene tre o quattro lettere. http://en.wikipedia.org/wiki/File format

G

Gestione del ciclo di vita Insieme delle regole, delle procedure e delle attività utilizzate per gestire i documenti sia cartecei che elettronici durante tutto l'arco della loro vita. Le principali ripercussioni della gestione del ciclo di vita delle risorse digitali, qualunque sia la loro forma o 
funzione, si riferiscono alla necessità di gestire attivamente la risorsa in ogni fase del suo ciclo di vita e al riconoscimento immediato/tempestivo delle interdipendenze esistenti tra ogni fase e le fasi iniziali di conservazione. Ciò rappresenta la differenza principale con gran parte della conservazione tradizionale in cui la gestione è prevalentemente passiva fino a quando non necessita di un lavoro articolato di conservazione, generalmente diversi anni dopo la creazione, e, raramente, se mai dovesse accadere, coinvolgendo il creatore. Esiste un ciclo di vita attivo e interconnesso per le risorse digitali che ha indotto molti a favorire il termine 'continuo' per distinguerlo dal flusso più tradizionale e lineare del ciclo di vita delle risorse analogiche tradizionali. II termine ciclo di vita è stato usato qui come applicazione di un concetto proattivo di gestione della conservazione per le risorse digitali.

Gestione della conservazione digitale Workshop e Tutorial Un workshop di formazione intensivo e un tutorial online sviluppato e tenuto dalla Cornell University Library, 2003-2006; esteso e tenuto da CPSR, 2007-2012; e ora esteso e tenuto da MIT Libraries, 2012-on. http://dpworkshop.org/

GIF Graphic Interchange Format, un'immagine che usa generalmente una compressione di dati lossy. http://en.wikipedia.org/wiki/GIF

Gigabyte (GB) Un'unità di informazione digitale che si usa spesso per descrivere i dati o le dimensioni di archiviazione dei dati, equivale approssimativamente a 1,000 Megabytes (MB).

GIS Geographical Information System, un sistema che processa insieme i mapping e i dati.

\section{H}

HTML Hypertext Markup Language, un formato usato per presentare un testo e altre informazioni sul World Wide Web. Le versioni della specifica HTML sono state mantenute sin dal 1996 dal Consorzio W3C, World Wide Web Consortium (W3C). http://en.wikipedia.org/wiki/HTML

IASA International Association of Sound and Audiovisual Archives, un'associazione per archivi che conserva gli audio registrati e i documenti audiovisivi. http://www.iasa-web.org

IIPC The International Internet Preservation Consortium. http://www.netpreserve.org

Informatica forense L'applicazione di metodologie e strumenti scientifico-tecnici in una prospettiva di conservazione, collezione, validazione, identificazione, analisi, interpretazione, documentazione e presentazione delle informazioni digitali ottenute dalle risorse digitali in un momento successivo.

Ingest il processo di trasformazione di un Submission Information Package (SIP) in un Archival Information Package (AIP), ad es., inserimento di dati in un archivio digitale (termine $\underline{\mathrm{OAIS}}$ ).

InterPARES progetto International Research on Permanent Authentic Records in Electronic Systems. http://www.interpares.org 
ISO International Organization for Standardization. http://www.iso.org/iso/home.html

JHove2 Uno strumento di caratterizzazione degli oggetti digitali. La caratterizzazione comprende quattro elementi: identificazione del formato dell'oggetto; convalida che l'oggetto sia conforme alle norme tecniche del suo formato; estrazione dei metadati dall'oggetto; valutazione se l'oggetto deve essere accettato o meno nel repository basandosi sulle politiche stabilite dal curatore. https://bitbucket.org/ihove2/main/wiki/Home

JPEG Joint Photographic Experts Group, una commissione che supervisiona gli standard internazionali per la compressione e processamento delle fotografie digitali. La maggior parte dei formati JPEG è lossy. http://www.jpeg.org/

JPEG 2000 una revisione del formato JPEG format che può usare la compressione senza perdita.

\section{K}

Kilobyte (KB) Un'unità di informazione digitale usata per descrivere i dati o la dimensione di archiviazione dei dati, equivale approssimativamente a 1,000 Bytes.

$\mathbf{L}$

LOTAR (LOng Term Archiving and Retrieval), uno standard della conservazione digitale per $\mathrm{i}$ modelli 3D CAD e per le informazioni relative ai gestori dei dati di prodotto sviluppati dall'internazionale LOTAR, un consorzio industriale aerospaziale e di compagnie di difesa dall'America e dall'Europa. http://www.lotar-international.org

M

Megabyte (MB) Un'unità di informazione digitale usata per descrivere i dati o la dimensione di archiviazione dei dati, equivale approssimativamente a 1,000 Kilobytes (KB).

Metadati Informazioni che descrivono aspetti significativi di una risorsa. Attualmente si tende a dare molta importanza ai metadati ai fini del rilevamento delle risorse. Questo Manuale si concentra sulla necessità dei metadati per gestire e conservare con successo le risorse digitali nel tempo e che saranno di supporto per garantire le informazioni essenziali contestuali, storiche e tecniche conservate insieme all'oggetto digitale. II PREMIS Data Dictionary per i Metadati di Conservazione è diventato di fatto uno standard di riferimento nel settore della conservazione digitale.

METS Metadata Encoding and Transmission Standard, uno standard per la presentazione dei metadati con l'utilizzo di XML. http://www.loc.gov/standards/mets/

Migrazione Un modo per superare l'obsolescenza tecnologica trasferendo le risorse digitali da una generazione hardware/software a una successiva. L'obiettivo della migrazione è quello di conservare il contenuto intellettuale degli oggetti digitali e di mantenere per gli utenti la capacità di raccoglierli, presentarli, o altrimenti utilizzarli facendo fronte al costante sviluppo tecnologico. 
La migrazione è diversa dal riaggiornamento dei supporti di archiviazione poiché non è sempre possibile realizzare una copia digitale esatta o replicare le caratteristiche e l'aspetto dell'originale e mantenere comunque la compatibilità della risorsa con una tecnologia di nuova generazione.

MIME Multipurpose Internet Mail Extensions. Un protocollo per includere le informazioni nonASCII nei messaggi di posta elettronica. I software generalmente comprendono degli strumenti che fungono da interprete che convertono, ove necessario, il contenuto MIME nel e dal suo formato nativo. http://en.wikipedia.org/wiki/MIME

MPEG Moving Picture Experts Group. Una commissione responsabile dello sviluppo di standard internazionali per la compressione, decompressione, processamento, e rappresentazione codificata delle immagini in movimento, dell'audio e della loro combinazione. https://mpeg.chiariglione.org

Nativi digitali Le risorse digitali che non sono destinate ad avere un equivalente analogico, sia come risorsa originaria che come risultato di conversione da una versione analogica. Questo termine è stato usato nel Manuale per differenziarle da 1) risorse digitali che sono state create come risultato di conversione degli originali analogici; e 2) risorse digitali che potrebbero essere state create da una risorsa digitale, ma che sono state stampate su carta, ad es., alcuni documenti elettronici.

NCDD The Netherlands Coalition for Digital Preservation. http://www.ncdd.nl/en/

NDSA National Digital Stewardship Alliance, un'organizzazione US attiva nella conservazione digitale. http://www. digitalpreservation.gov/ndsa/

NESTOR La rete di competenza tedesca per la conservazione digitale. http://www.langzeitarchivierung.de/Subsites/nestor/EN/Home/home node.html/

\section{0}

Open Archival Information System (OAIS) Standard ISO 14721:2012 che definisce concetti, modelli e funzionalità del processo di conservazione delle risorse digitali. Un archivio che consiste in un'organizzazione, che a sua volta può appartenere a una più grande, fatta di persone e sistemi, che si è assunto la responsabilità di conservare le informazioni e renderle disponibili per una Comunità designata. Rispetta una serie di requisiti, così come definito nella sezione 4 dello standard OAIS. L'abbreviazione OAIS viene anche comunemente usata per riferirsi al modello standard di riferimento Open Archival Information System che ne definisce il termine. Lo standard è una struttura concettuale che descrive l'ambiente, i componenti funzionali, e gli oggetti informativi associati a un sistema responsabile della conservazione a lungo termine. Essendo un modello di riferimento, il suo obiettivo principale è quello di fornire un insieme generale di concetti e definizioni che possono sostenere la discussione tra i settori e i gruppi professionali e facilitare la definizione delle specifiche degli archivi e dei sistemi di conservazione digitale. Ha un set di base di disposizioni di conformità che dovrebbero essere considerate essenziali. OAIS è stato per la prima volta approvato come ISO Standard 14721 nel 2002 e nel 2012 è stata pubblicata una seconda edizione. Anche se realizzato sotto la 
direzione della Consultative Committee for Space Data Systems ( $\underline{\text { CSDS}}$ ), ha ricevuto importanti input da biblioteche e archivi.

OPF Open Preservation Foundation, originariamente Open Planets Foundation. http://openpreservation.org

\section{$\mathbf{P}$}

PAIMAS Space Data and Information Transfer Systems - Producer-Archive Interface Methodology Abstract Standard. Questo standard ISO 20652:2006 tratta i primi passaggi del processo ingest definito dal modello di riferimento http://www.iso.org/iso/iso catalogue/catalogue tc/catalogue detail.htm?csnumber=39577

PDF Portable Document Format, un insieme di formati e standard aperti gestiti dalla International Organization for Standardization per la produzione e condivisione di documenti elettronici originariamente sviluppati da Adobe Systems. II formato di pagina descrittiva originale è stato elaborato nelle versioni successive per permettere l'integrazione di oggetti complessi come immagini, audio, file di immagini in movimento, collegamenti ipertestuali, metadati XML incorporati, e moduli aggiornabili. Le specificazioni per le diverse versioni e i profili del formato ora sono gestiti dalla International Standards Organization. http://www.adobe.com/uk/products/acrobat/adobepdf.html

PDF/A Versione dello standard PDF destinata a uso archivistico. http://www.aiim.org/Researchand-Publications/Standards/Committees/PDFA

PDI Preservation Description Information. Le informazioni che sono necessarie per una conservazione appropriata del Contenuto informativo e che possono essere categorizzate come



Petabyte (PB) Un'unità di informazione digitale spesso usata per descrivere i dati o la dimensione di archiviazione dei dati, equivale approssimativamente a 1,000 Terabytes (TB)

PIN Pérennisation des Informations Numériques, II gruppo nazionale francese per la conservazione digitale. http://pin.association-aristote.fr/doku.php

PREMIS Preservation Metadata: Implementation Strategies. Uno standard di fatto per i metadati di conservazione digitale. http://www.loc.gov/standards/premis/

PRONOM Un database di formati dei file, prodotti software e altri componenti tecnici necessari per supportare l'accesso a lungo termine ai documenti elettronici e ad altri oggetti digitali di valore culturale, storico 0 aziendale. Usato con DROID. http://apps.nationalarchives.gov.uk/PRONOM/Default.aspx

Proprietà significative Caratteristiche degli oggetti digitali e intellettuali che devono essere conservate nel tempo per garantire l'accessibilità continua, la fruibilità e la significatività degli oggetti e la capacità di essere accettati (evidenze di) per ciò che pretendono di essere. https://www.archives.gov/files/era/acera/pdf/significant-properties.pdf 
PST Personal Storage Table, estensione di file per 'archivi personali' elaborata con il programma Microsoft Outlook. I file PST contengono messaggi di posta elettronica e voci agenda utilizzando un formato proprietario ma aperto e potrebbero essere trovati nei dispositivi locali o interconnessi alle e-mail degli utenti finali. Esistono diversi strumenti in grado di leggere i file PST e di migrarli verso altri formati. http://en.wikipedia.org/wiki/Personal Storage Table

Pubblicazioni digitali "Native digitali" oggetti che sono stati pubblicati per l'accesso pubblico, resi anche disponibili e distribuiti gratuitamente o a pagamento. Possono essere costituiti da pubblicazioni interconnesse, disponibili su reti di comunicazioni o pubblicazioni su formati fisici che sono distribuite su supporti come floppy disk o dischi ottici. Possono essere sia statici che dinamici.

Riformattazione Copiare il contenuto informativo da un supporto di archiviazione a un altro (riformattazione dei media) o la conversione da un formato di file a uno differente (riformattazione dei file).

Risorse digitali Un termine generale che racchiude le copie digitali create come risultato della conversione delle risorse analogiche in formato digitale (digitalizzazione), i 'nativi digitali' per i quali la conversione non si è mai verificata e che non sono destinati ad avere un equivalente analogico, e i documenti digitali.

\section{S}

Sandbox Un ambiente sicuro di calcolo per eseguire codici nuovi, non testati o sperimentali, o cambiamenti nel codice, compresi i codici potenzialmente malevoli. L'ambiente è autonomo con risorse strettamente monitorate ed è tipicamente virtuale.

SGML Standard Generalized Markup Language. uUo standard ISO su come specificare il linguaggio di markup di un documento 0 il suo insieme di tag. http://en.wikipedia.org/wiki/Standard Generalized Markup Language

SIP Submission Information Package. Un pacchetto informativo che viene fornito dal produttore dell'OAIS per essere usato per la realizzazione o l'aggiornamento di uno o più Archival Information Packages ( $\underline{\text { AIPs }}$ ) e/o dell'associata Descriptive Information (termine OAIS).

SMPTE Society of Motion Picture and Television Engineers, organizzazione professionale e organismo di standard tecnici per la televisione e il cinema. https://www.smpte.org

\section{$\mathbf{T}$}

TDR Trusted Digital Repository. Per repository digitale affidabile si intende 'una missione per fornire alla propria comunità designata ora e nel futuro un accesso affidabile a lungo termine a risorse digitali gestite'. La TDR deve comprendere i seguenti sette attributi: conformità con il

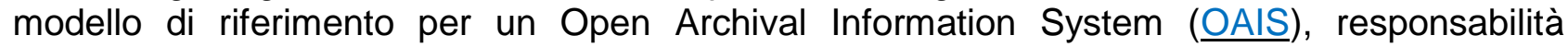
amministrativa, fattibilità organizzativa, sostenibilità finanziaria, adeguatezza tecnologica e 
procedurale, sicurezza dei sistemi e responsabilità procedurale. II concetto è stato particolarmente importante per quanto riguarda la certificazione di repository digitali.

Terabyte (TB) Un'unità di informazione digitale spesso usata per descrivere i dati o la dimensione diarchiviazione dei dati, ed equivale approssimativamente a 1,000 Gigabytes (GB).

Three-Legged Stool Un approccio concettuale alla conservazione digitale che indica un programma pienamente implementato ed eseguibile di conservazione digitale che riguarda aspetti organizzativi, questioni tecnologiche e di finanziamento, bilanciandole come un threelegged stool. Sviluppato come parte del Digital Preservation Management Workshop and Tutorial.

TIFF Tagged Image File Format, formato comune per immagini tipicamente lossless. http://en.wikipedia.org/wiki/Tagged Image File Format

TRAC Trusted Repository Audit and Certification, toolkit per controllare un repository digitale. http://www.crl.edu/sites/default/files/d6/attachments/pages/trac 0.pdf

\section{$\mathbf{U}$}

UKWA Archivio web UK http://www.webarchive.org.uk/ukwa/

V

W

WARC II format WARC (Web ARChive) è un formato contenitore per i siti web archiviati, anche conosciuto come ISO 28500:2009. È una revisione dell'Internet Archive's ARC File Format usato per archiviare i web crawls raccolti dal World Wide Web. http://www.iso.org/iso/iso catalogue/catalogue tc/catalogue detail.htm?csnumber=44717

WAV il file wrapper standard per gli audio; si veda BWF (Broadcast WAV Format) per la versione professionale. http://en.wikipedia.org/wiki/WAV

\section{$\mathbf{X}$}

XML Extensible Markup Language, uno standard ampiamente utilizzato (deriva da SGML), per la presentazione di informazioni strutturate, tra cui documenti, dati, configurazioni, libri e transazioni. È gestito dal W3C, World Wide Web Consortium (W3C). http://www.w3.org/XML/ 